Portland State University

PDXScholar

$4-15-2008$

\title{
Design of Radio-Frequency Filters and Oscillators in Deep-Submicron CMOS Technology
}

Haiqiao Xiao

Portland State University

Follow this and additional works at: https://pdxscholar.library.pdx.edu/open_access_etds

Part of the Electrical and Computer Engineering Commons Let us know how access to this document benefits you.

\section{Recommended Citation}

Xiao, Haiqiao, "Design of Radio-Frequency Filters and Oscillators in Deep-Submicron CMOS Technology" (2008). Dissertations and Theses. Paper 5233.

https://doi.org/10.15760/etd.7106

This Dissertation is brought to you for free and open access. It has been accepted for inclusion in Dissertations and Theses by an authorized administrator of PDXScholar. Please contact us if we can make this document more accessible: pdxscholar@pdx.edu. 


\section{DISSERTATION APPROVAL}

The abstract and dissertation of Haiqiao Xiao for the Doctor of Philosophy in

Electrical and Computer Engineering were presented April 15, 2008, and accepted by the dissertation committee and the doctoral program.

COMMITTEE APPROVALS:

Rolf Schaumann, Chair

W. Robert Daasch

Branimir Pejcinovic

Xiaoyu Song

Charles Balogh

Representative of the Office of Graduate Studies

DOCTORAL PROGRAM APPROVAL:

Malgorzata Chrzanowska-Jeske, Director

Electrical and Computer Engineering

Ph.D. Program 


\begin{abstract}
An abstract of the dissertation of Haiqiao Xiao for the Doctor of Philosophy in Electrical and Computer Engineering presented April 15, 2008.
\end{abstract}

Title: Design of Radio-Frequency Filters and Oscillators in Deep-Submicron CMOS Technology

Radio-frequency filters and oscillators are widely used in wireless communication and high-speed digital systems, and they are mostly built on passive integrated inductors, which occupy a relative large silicon area. This research attempted to implement filters and oscillators operating at 1-5 GHz using transistors only, to reduce the circuits' area. The filters and oscillators are designed using active inductors, based on the gyrator principle; they are fabricated in standard digital CMOS technology to be compatible with logic circuits and further lower the cost. To obtain the highest operating frequency, only parasitic capacitors were used.

Two new active-inductor circuits are derived from this research, labeled allNMOS and all-NMOS-II. The all-NMOS active inductor was used to design high- $Q$ bandpass filters and oscillators, which were fabricated in TSMC's $0.18-\mu \mathrm{m}$ digital CMOS process. The highest center frequency measured was $5.7 \mathrm{GHz}$ at $0.20-\mu \mathrm{m}$ gate length and the maximum repeatably measured $Q$ was $665.2 .4-\mathrm{GHz}$ circuits were also designed and fabricated in $0.40-\mu \mathrm{m}$ gate length. The all-NMOS-II circuit has superior linearity and signal fidelity, which are robust against process and temperature variations, due to its novel structure. It was used in signal drivers and will be 
fabricated in commercial products.

Small-signal analysis was conducted for each of the active-inductor, filter and oscillator circuits, and the calculated performance matches those from simulations. The noise performance of the active inductor, active-inductor filter and oscillator was also analyzed and the calculated results agree with simulations. The difference between simulation and measured results is about $10 \%$ due to modeling and parasitic extraction error.

The all-NMOS active-inductor circuit was granted a US patent. The US patent for all-NMOS-II circuit is pending. This research generated three conference papers and two journal papers. 


\title{
DESIGN OF RADIO-FREQUENCY
}

\section{FILTERS AND OSCILLATORS IN DEEP-SUBMICRON \\ CMOS TECHNOLOGY}

\author{
by \\ HAIQIAO XIAO
}

\begin{abstract}
A dissertation submitted in partial fulfillment of the requirements for the degree of

DOCTOR OF PHILOSOPHY

in

ELECTRICAL AND COMPUTER ENGINEERING
\end{abstract}

Portland State University

2008 
Copyright 2008

by

Haiqiao Xiao 


\section{ACKNOWLEDGMENTS}

First of all, I would like to thank my academic advisor, Professor Rolf Schaumann, for his guidance and insight on filter theory, analog circuits, and research. Through my working with him, my research skills, such as critical thinking and problem solving, improved tremendously. I also learnt ways to do a quality and thorough work and be creative.

I would like to thank Professor W. Robert Daasch for his advice and discussions on circuits, layout, technology and device modeling. His enthusiasm about knowledge is very encouraging, and I learnt how to pursue a problem to its root through working with him.

I thank Professor Branimir Pejcinovic for the many discussions on radiofrequency designs and measurements. His microwave course was very useful to my research and interesting by itself.

I would like to thank Professor Xiaoyu Song for his advice and discussions on integrated circuit design and research. His Digital IC Design course helped me greatly on CMOS technology and device physics. I was very much encouraged and affected by his optimism and resourcefulness in solving problems.

I thank Professor Paul Van Halen for his lectures on analog circuit design, which were insightful and helpful to my research.

I thank Philip K. Wong for his help on using the microwave instruments during 
IC laboratory characterization.

I thank Professors Charles Balogh and Mara Tableman for their lectures on mathematics. The knowledge is very beneficial to my career and broadened my interests. I appreciate Prof. Charles Balogh's acting as my Graduate Office Representative.

I would like to thank Joseph D. Stenger and Nathan Moyal of Cypress Semiconductors for their support and encouragement on the dissertation, and the opportunity they provided me to advance my research. The help of Jeff Kittle at Cypress on the measurement of phase noise is gratefully acknowledged.

I acknowledge the financial support from the Semiconductor Research Corporation (SRC), ECE Department of Portland State University, and Cypress Semiconductors during my study.

I especially thank my wife, Jing Xia, for her encouragement and patience on my study and research. Without her support, I would not be able to complete the dissertation. I am deeply indebted to her for allowing me to devote so much time and attention to academic pursuit.

I thank my parents and sister for their understanding and encouragement, and for helping me choosing the right career.

I owe special thanks to Hans and Lois Blom, for their encouragement and spiritual inspiration to enable the completion of the dissertation. Lois' proofreading of my dissertation is greatly appreciated.

Finally, I thank Xiangying Ma, Wei Wang, David H. Chiang and Aydin Karsilayan for introducing me to such a great study opportunity. 


\section{TABLE OF CONTENTS}

page

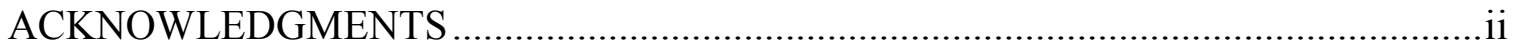

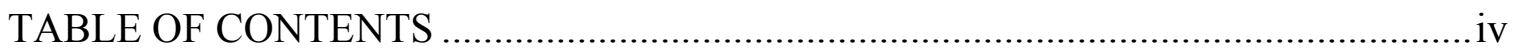

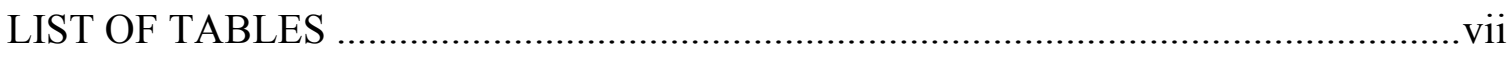

LIST OF FIGURES ....................................................................................... viii

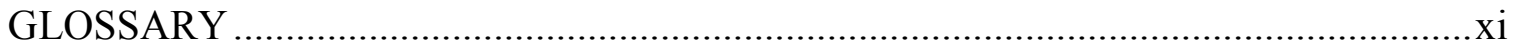

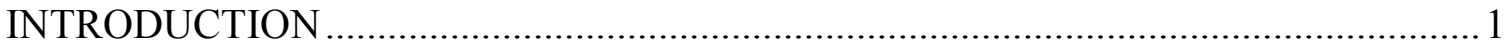

1.1 The Need for RF Analog Filters and Oscillators........................................... 1

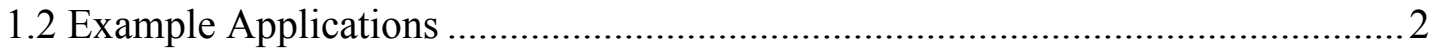

1.3 Implementation Methods and Literature Survey ............................................ 4

1.4 Technical Requirements Summary ......................................................... 7

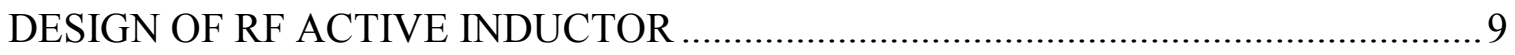

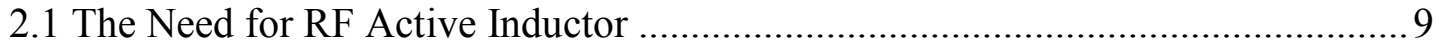

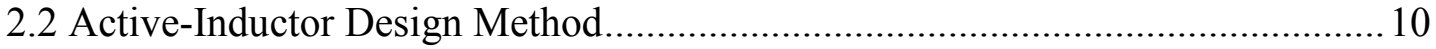

2.2.1 The Gyrator Approach .................................................................... 10

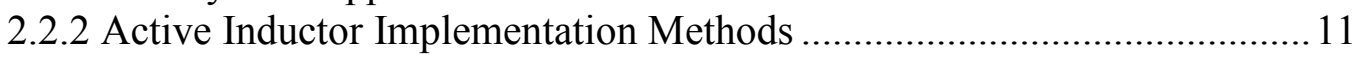

2.2.3 The Active-Inductor Equivalent Circuit.............................................. 15

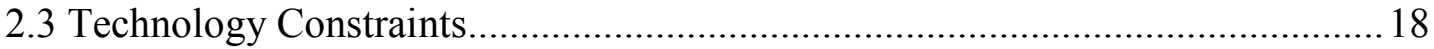

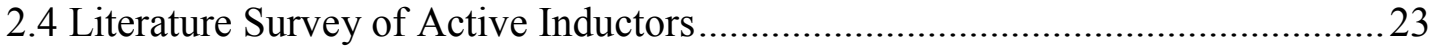

2.4.1 Gate- $R$ and Gate- $R$-II Active Inductors................................................. 24

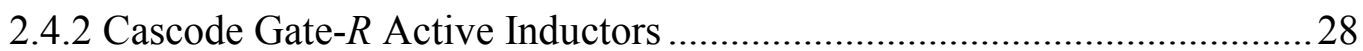

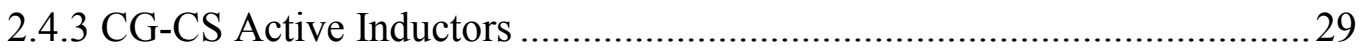

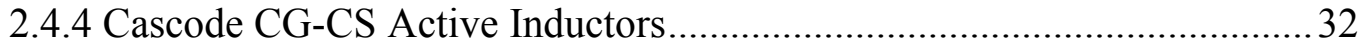

2.4.5 CS-CD Active Inductor and $Q$ Enhancement using $R_{\mathrm{f}} \ldots \ldots \ldots \ldots \ldots \ldots \ldots \ldots . . . . . . . . . . . .34$

2.4.6 Cascode and Regulated-Cascode Active Inductors...................................38

2.4.7 Differential-Pair Active Inductor ......................................................... 42

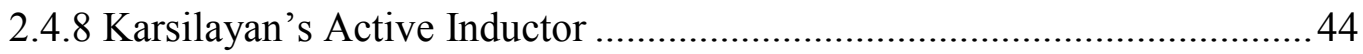

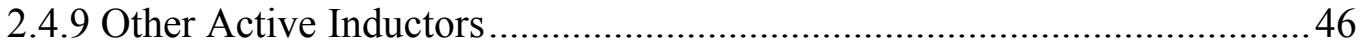

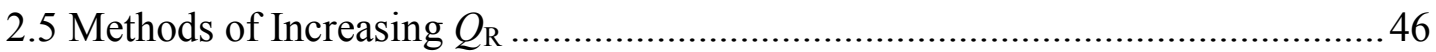

2.5.1 Reducing $g_{1}$ and/or $g_{\mathrm{L}}$ in Gyrator Equivalent Circuit ............................. 46

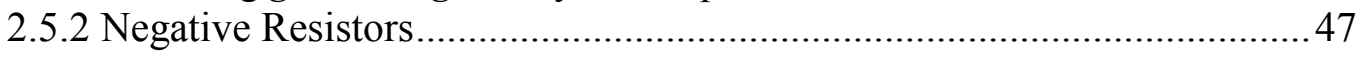

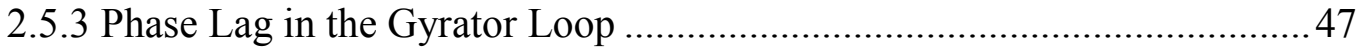

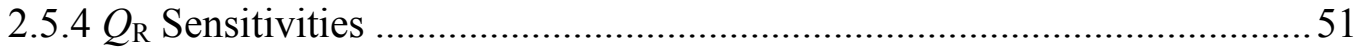


2.6 Noise of Gyrator-Based Active Inductors ........................................................52

2.7 The All-NMOS Active Inductor (New in This Work) ........................................56

2.7.1 The All-NMOS Active-Inductor Circuit................................................56

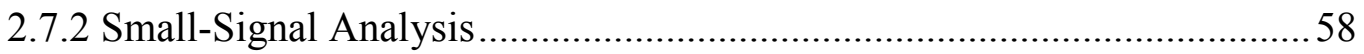

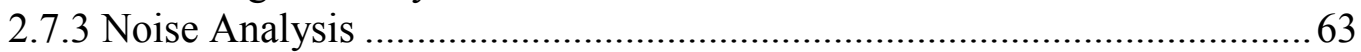

2.8 All-NMOS-II Active-Inductor Circuit (New in This Work)................................70

2.8.1 The Circuit as a Precision Analog Signal Driver ....................................... 70

2.8.2 Analysis using Feedback Theory …………………............................... 73

2.8.3 AC Small-Signal Analysis ………………………….............................. 75

2.8.4 Design Example .................................................................................. 79

2.8.5 The all-NMOS-II Circuit Configured as an Active Inductor .......................83

2.8.6 Example of the all-NMOS-II Active Inductor Circuit ................................8 87

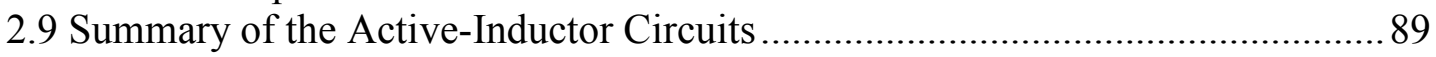

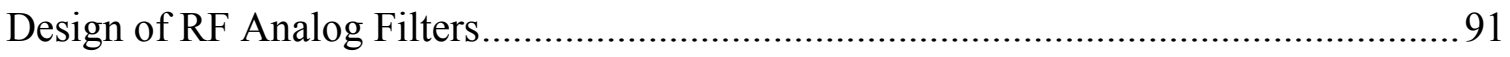

3.1 Designing RF Analog Filters with Active Inductors.............................................91

3.1.1 Bandpass Filters ............................................................................ 91

3.1.2 Lowpass Filters ……………………………………………….... 93

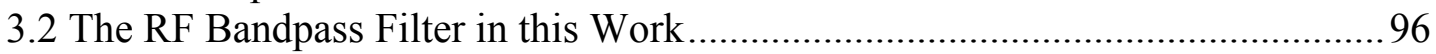

3.2.1 The Bandpass Filter Circuit ……………………………………….......96

3.2.2 Noise Analysis of the Bandpass Filter .................................................. 98

3.2.3 Bandpass Filter Example.................................................................... 100

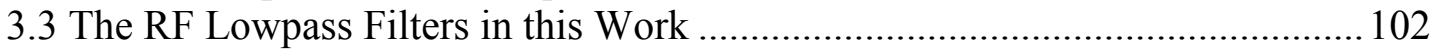

3.3.1 All-NMOS-II Active Inductor Lowpass Filter.......................................... 102

3.3.2 All-NMOS Active Inductor Lowpass Filter................................................ 103

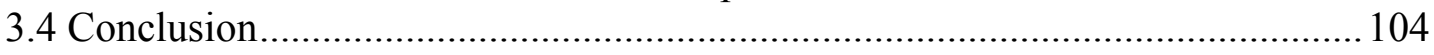

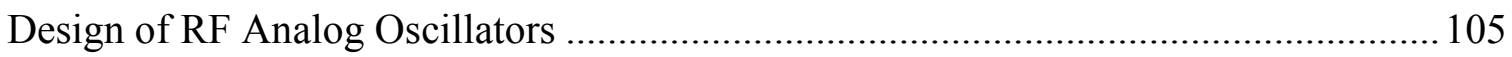

4.1 Designing RF Oscillators with Active Inductors .................................................. 105

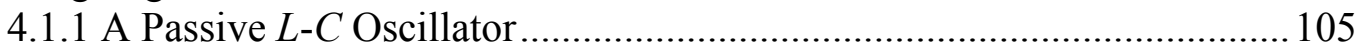

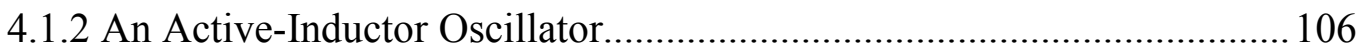

4.1.3 Reducing Output Voltage Harmonic Distortion ....................................... 107

4.1.4 Designing the Oscillator Loop Gain ....................................................... 110

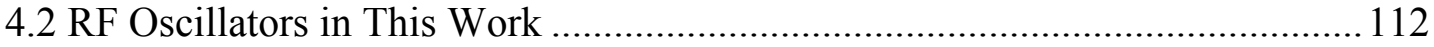

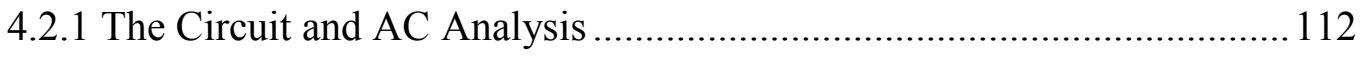

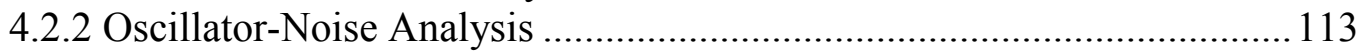

4.2.3 Example of Active-Inductor Oscillator ................................................... 119

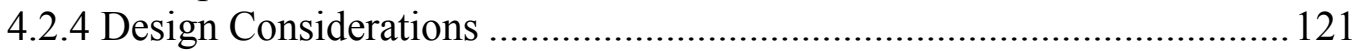

4.2.5 Simulation Results and Analysis........................................................... 122

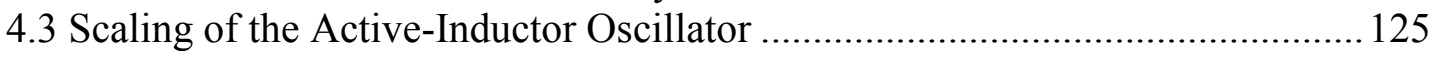

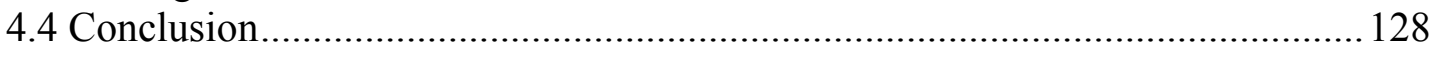

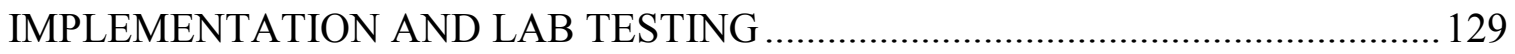




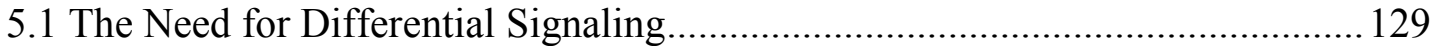

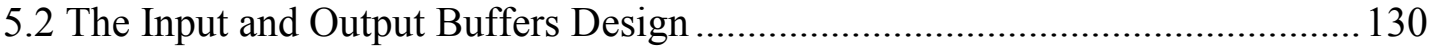

5.2.1 The Need for Input and Output Buffers ............................................... 130

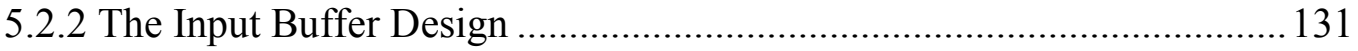

5.2.3 The Output Buffer Design.................................................................... 134

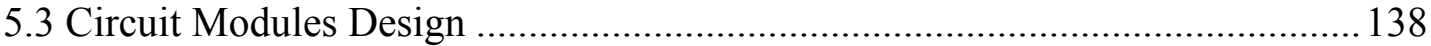

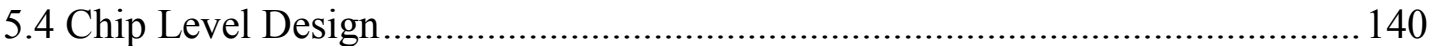

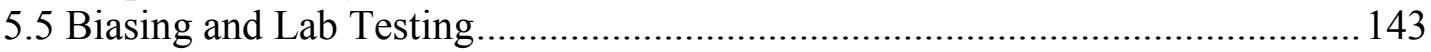

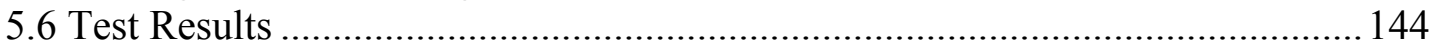

5.6.1 5-GHz Active Inductor Oscillator (OSC $0.2, L=0.2 \mu \mathrm{m}) \ldots \ldots \ldots \ldots \ldots \ldots \ldots \ldots . . .144$

5.6.2 The 2.6-GHz Active Inductor Oscillator (OSC0.4, $L=0.4 \mu \mathrm{m}) \ldots \ldots \ldots \ldots . . .148$

5.6.3 The High- $Q$ Active-Inductor Bandpass Filters ........................................ 151

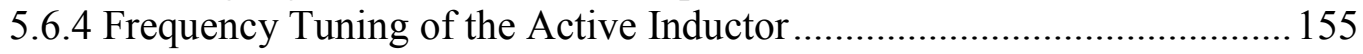

5.6.5 Measured Frequency Statistics............................................................... 157

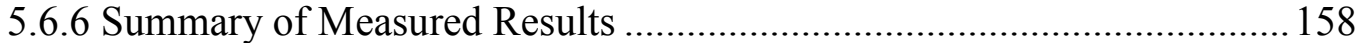

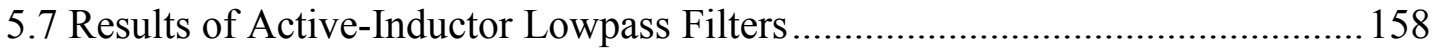

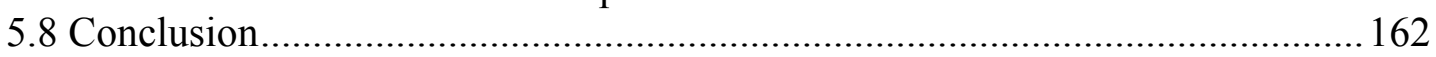

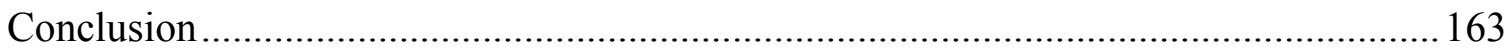

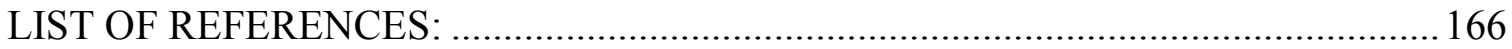




\section{LIST OF TABLES}

$\underline{\text { Table }}$

page

TABLE 2-1 CALCULATED VERSUS SIMULATED RESULTS FOR THE ALL-NMOS ACTIVE INDUCTOR. .63

TABLE 2-2 ALL-NMOS ACTIVE INDUCTOR NOISE SUMMARY …….......................................69

TABLE 2-3 CALCULATED VERSUS SIMULATED RESULTS FOR THE ALL-NMOS-II CIRCUIT WHEN USED AN ANALOG SIGNAL DRIVER OR LOWPASS FILTER …………...................8

TABLE 2-4 CALCULATED VERSUS SIMULATED RESULTS FOR THE ALL-NMOS-II CIRCUIT

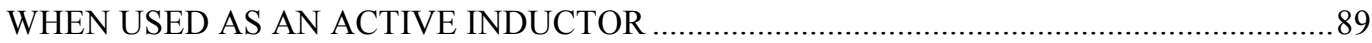

TABLE 2-5 SUMMARY OF ACTIVE-INDUCTOR CIRCUITS........................................................ 90

TABLE 3-1 CALCULATION OF ACTIVE-INDUCTOR BANDPASS PERFORMANCES ...............102

TABLE 4-1 CALCULATED VERSUS SIMULATED OSCILLATOR PARAMETERS .....................125

TABLE 5-1 PERFORMANCE SUMMARY OF THE ACTIVE-INDUCTOR FILTER AND COMPARISON

TABLE 5-2 FREQUENCY $\left(F_{0}\right)$ STATISTICS OF THE OSCILLATORS. ..................................... 158

TABLE 5-3 SUMMARY OF MEASURED RESULTS 158 


\section{LIST OF FIGURES}

$\underline{\text { Figure }}$

page

FIGURE 1-1 THE ARCHITECTURE OF A WIRELESS COMMUNICATION IC ……........................

FIGURE 1-2 BLOCK DIAGRAM OF A CHARGE-PUMP PLL ……….........................................

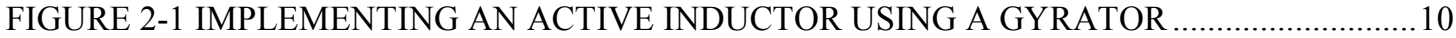

FIGURE 2-2 IMPLEMENTING AN ACTIVE INDUCTOR USING TWO OPAMPS. .........................12

FIGURE 2-3 IMPLEMENTING AN ACTIVE INDUCTOR USING OTAS OR $G_{\mathrm{M}} \mathrm{S} \ldots \ldots \ldots \ldots \ldots \ldots \ldots \ldots \ldots . . . . . . . . . . . . . .13$

FIGURE 2-4 EQUIVALENT CIRCUIT OF A $G_{\mathrm{M}^{-}} C$ ACTIVE INDUCTOR ......................................14

FIGURE 2-5 SIMULATION PLOTS OF THE CIRCUIT IN FIG. 2-4 ................................................. 18

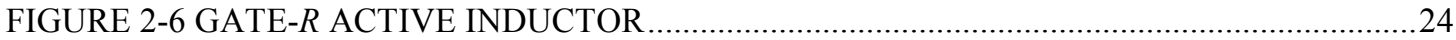

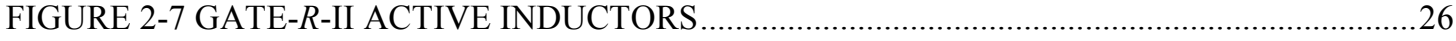

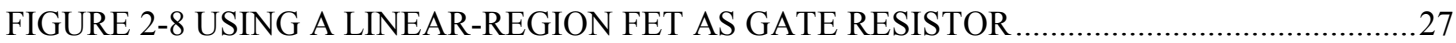

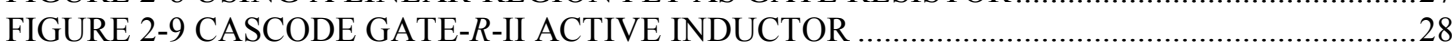

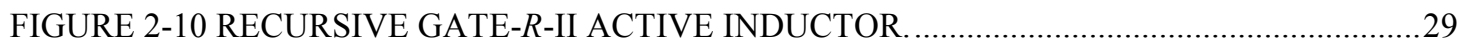

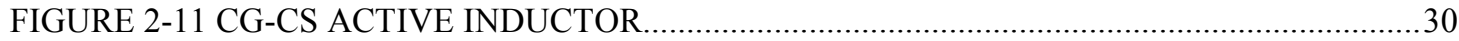

FIGURE 2-12 DERIVATION OF THE CASCODE CG-CS ACTIVE INDUCTOR .................................33

FIGURE 2-13 WU AND ISMAIL'S SOLUTION TO DC BIASING DIFFICULTY ...............................34

FIGURE 2-14 COMMON-SOURCE COMMON-DRAIN (CS-CD) ACTIVE INDUCTOR ..................35

FIGURE 2-15 A CS-CD ACTIVE INDUCTOR SIMULATION CIRCUIT ….......................................37

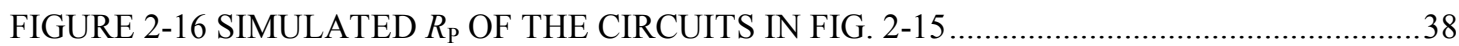

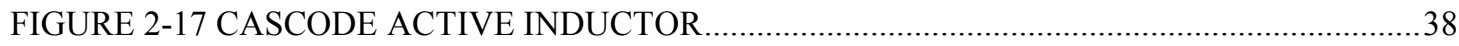

FIGURE 2-18 ADDING $R_{\mathrm{B}}$ TO THE CASCODE ACTIVE INDUCTOR ...........................................4

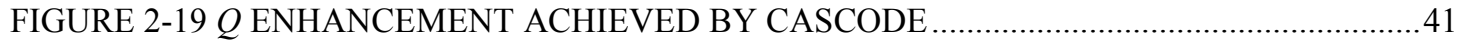

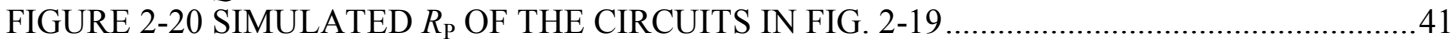

FIGURE 2-21 REGULATED CASCODE ACTIVE INDUCTOR CIRCUIT …....................................42

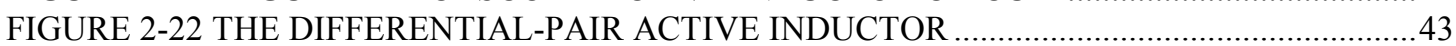

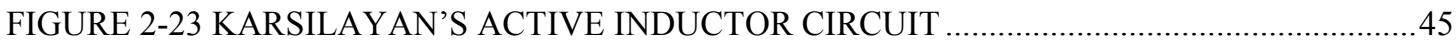

FIGURE 2-24 THE GYRATOR EQUIVALENT CIRCUIT OF AN ACTIVE INDUCTOR ................48

FIGURE 2-25 THE NOISE MODEL OF AN ACTIVE INDUCTOR ..............................................53

FIGURE 2-26 A GYRATOR-BASED ACTIVE INDUCTOR SHOWING NOISE ELEMENTS..........53

FIGURE 2-27 THE ALL-NMOS SIGNAL-PATH ACTIVE-INDUCTOR CIRCUIT............................57

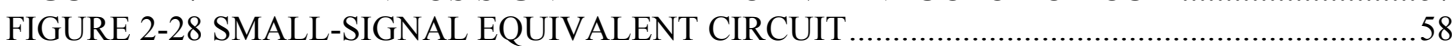

FIGURE 2-29 EXAMPLE SIMULATIONS OF ALL-NMOS ACTIVE INDUCTOR …......................61

FIGURE 2-30 THE NOISE MODEL OF A PSEUDO-DIFFERENTIAL ACTIVE INDUCTOR .........63

FIGURE 2-31 THE EQUIVALENT OUTPUT NOISE CURRENTS OF THE ALL-NMOS ACTIVE

INDUCTOR

. .64

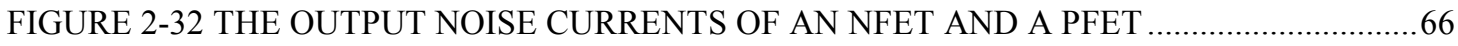

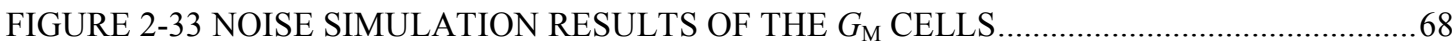

FIGURE 2-34 THE NOISE OF THE ACTIVE INDUCTOR SHOWN IN FIG. 2-29...........................69

FIGURE 2-35 THE ALL-NMOS-II CIRCUIT AS A PRECISION ANALOG SIGNAL DRIVER.......71

FIGURE 2-36 THE LOCAL FEEDBACK LOOP WITHIN THE CIRCUIT ……..................................74

FIGURE 2-37 THE MAIN FEEDBACK LOOP WITHIN THE CIRCUIT............................................74

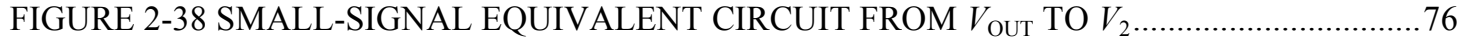

FIGURE 2-39 SMALL-SIGNAL EQUIVALENT CIRCUIT OF THE ENTIRE CIRCUIT ....................77

FIGURE 2-40 EXAMPLE OF ALL-NMOS-II CIRCUIT AS AN ANALOG SIGNAL DRIVER .........79

FIGURE 2-41 THE FREQUENCY RESPONSE OF THE ANALOG SIGNAL DRIVER .....................79

FIGURE 2-42 DC CHARACTERISTICS OF THE ANALOG SIGNAL DRIVER ..................................

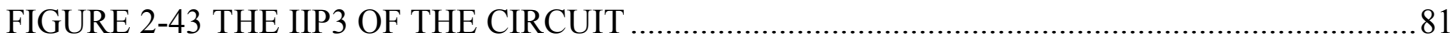

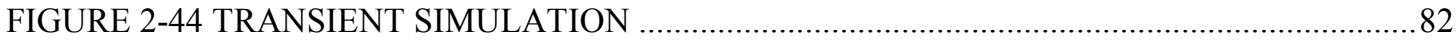

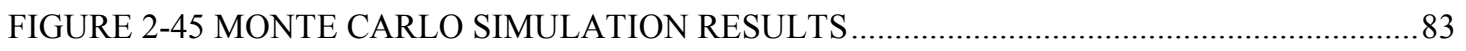


FIGURE 2-46 THE ALL-NMOS-II CIRCUIT AS AN ACTIVE INDUCTOR ...................................... 84

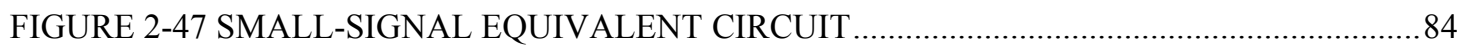

FIGURE 2-48 LATERAL AND VERTICAL DEVELOPMENT OF ANALOG CIRCUITS..................87

FIGURE 2-49 THE ALL-NMOS-II CIRCUIT CONFIGURED AS AN ACTIVE INDUCTOR...........87

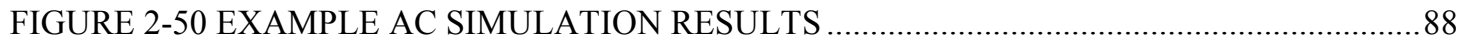

FIGURE 3-1 CONSTRUCTING AN HF ACTIVE-INDUCTOR BANDPASS FILTER ......................92

FIGURE 3-2 IMPLEMENTING A LOWPASS FILTER USING THE ACTIVE INDUCTOR ............94

FIGURE 3-3 REUSING $G_{\mathrm{M} 1}$ AS INPUT $G_{\mathrm{M}}$ TO IMPLEMENT A LOWPASS FILTER ….................96

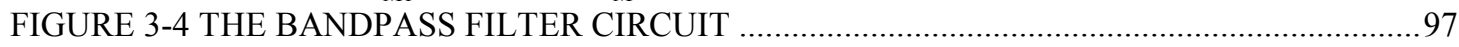

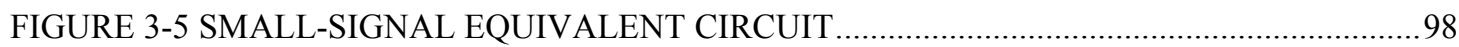

FIGURE 3-6 ACTIVE-INDUCTOR BANDPASS FILTER EXAMPLE ………................................. 100

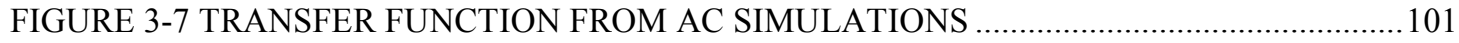

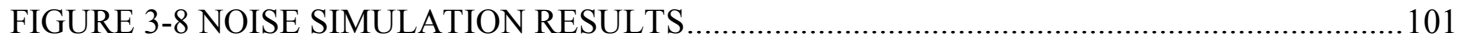

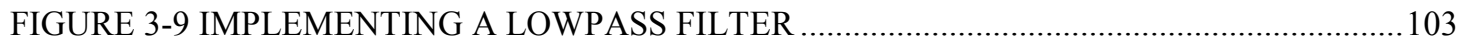

FIGURE 3-10 ANOTHER METHOD OF IMPLEMENTING A LOWPASS FILTER …....................104

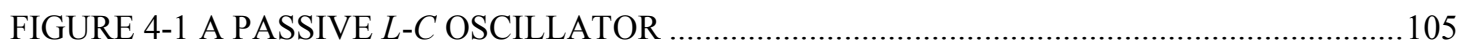

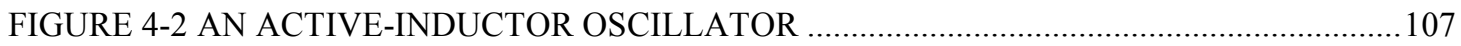

FIGURE 4-3 ACTIVE-INDUCTOR OSCILLATOR BLOCK DIAGRAM ........................................108

FIGURE 4-4 THE MAGNITUDE AND PHASE OF AN $R$ - $L$-C RESONATOR ................................111

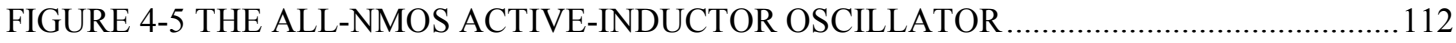

FIGURE 4-6 A TYPICAL OSCILLATOR PHASE-NOISE PROFILE ..............................................114

FIGURE 4-7 THE EQUIVALENT CIRCUIT OF THE ACTIVE-INDUCTOR OSCILLATOR .........116

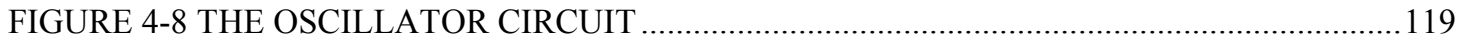

FIGURE 4-9 THE LIMITING EFFECT OF THE DIFFERENTIAL-PAIR NEGATIVE RESISTOR 120

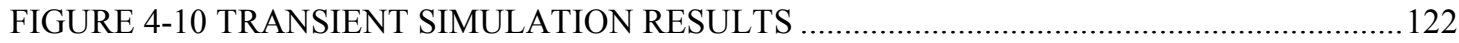

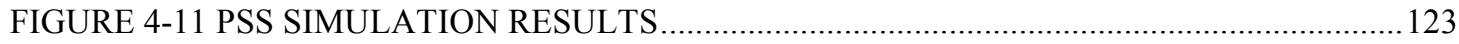

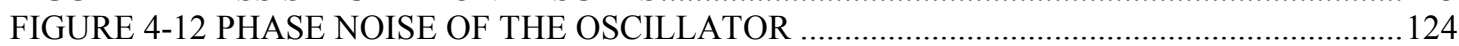

FIGURE 4-13 SIMULATION RESULTS OF THE $L=0.4 \mu \mathrm{M}$ OSCILLATOR (OSC0.4) ................127

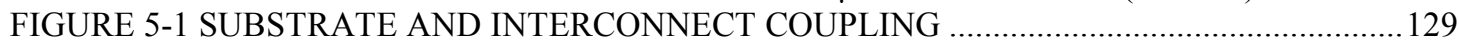

FIGURE 5-2 THE NEED FOR INPUT AND OUTPUT BUFFERS/DRIVERS ….............................131

FIGURE 5-3 THE SIGNAL PATH OF SINGLE-ENDED TO DIFFERENTIAL CONVERSION .....132

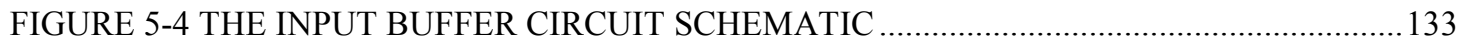

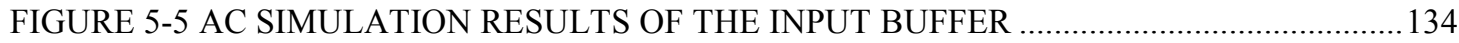

FIGURE 5-6 (A) A SINGLE GAIN STAGE. (B) CASCADED GAIN STAGES.................................135

FIGURE 5-7 THE FREQUENCY RESPONSE OF THE CIRCUIT IN FIG. 5-6(A) ..........................136

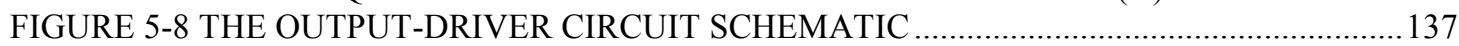

FIGURE 5-9 THE SIMULATION RESULTS OF THE OUTPUT DRIVER. ……............................138

FIGURE 5-10 REGULAR- $Q$ 0.2- $\mu$ M-GATE-LENGTH BANDPASS-FILTER MODULE (BP0.2).. 139

FIGURE 5-11 THE 0.2- $\mu$ M-BANDPASS-FILTER (BP0.2) MODULE LAYOUT ………………......... 139

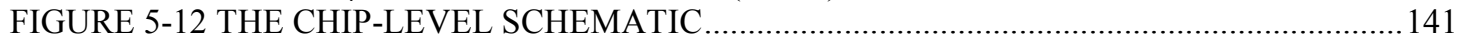

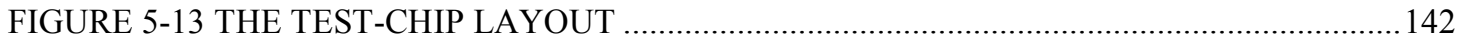

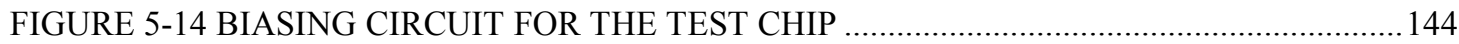

FIGURE 5-15 OUTPUT SPECTRUM OF A 5.6-GHZ OSCILLATOR............................................145

FIGURE 5-16 MEASURED PHASE NOISE OF THE 5-GHZ OSCILLATOR …..............................145

FIGURE 5-17 TEST-BENCH CONFIGURATION FOR THE 5-GHZ OSCILLATOR......................146

FIGURE 5-18 SIMULATION RESULTS OF THE FABRICATED 5-GHZ OSCILLATOR..............146

FIGURE 5-19 A MINOR DESIGN FLAW IN THE FABRICATED OSCILLATOR .........................147

FIGURE 5-20 THE SIMULATED PHASE NOISE OF THE OSC0.2 CORE ……............................ 148

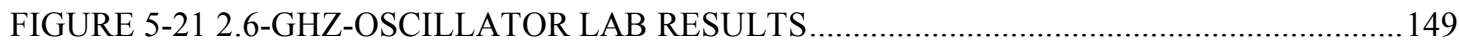

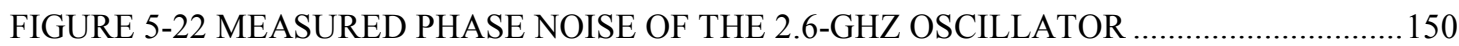

FIGURE 5-23 SIMULATION RESULTS OF THE 2.6-GHZ OSCILLATOR ……..........................150

FIGURE 5-24 MEASURED FREQUENCY RESPONSES OF THE BANDPASS FILTERS ............151 
FIGURE 5-25 MEASURED $Q$-TUNING CHARACTERISTIC OF THE 0.2- $\mu \mathrm{M}$ GATE-LENGTH

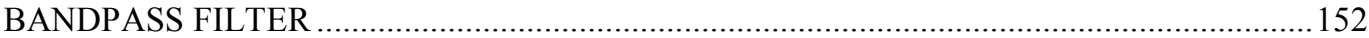

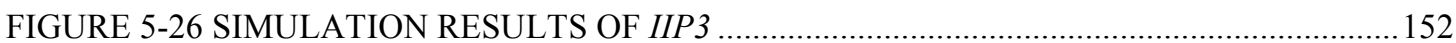

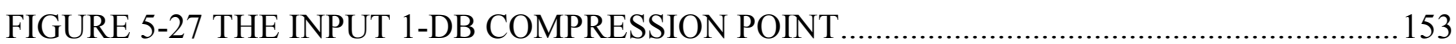

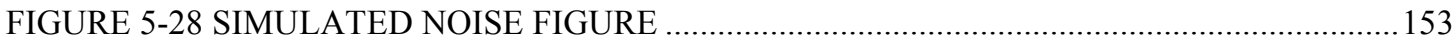

FIGURE 5-29 PLOT OF IIP3, INPUT 1-DB COMPRESSION POINT, AND EQUIVALENT OUTPUT NOISE 153

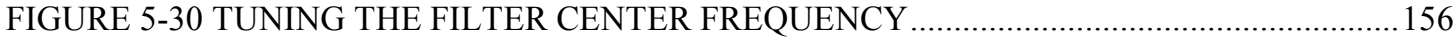

FIGURE 5-31 HISTOGRAMS OF OSCILLATOR $F_{0}$ AT NOMINAL BIASING ...............................157

FIGURE 5-32 MAGNITUDE AND PHASE RESPONSES OF THE MODULE LP0.2 …...................159

FIGURE 5-33 MAGNITUDE AND PHASE RESPONSES OF THE MODULE LP0.4 .......................160

FIGURE 5-34 MAGNITUDE AND PHASE RESPONSES OF THE REFERENCE CHANNEL.......161

FIGURE 5-35 LOWPASS FILTER FREQUENCY RESPONSE. 


\section{GLOSSARY}

ADC: Analog-to-digital converter/conversion.

ASIC: Application specific integrated circuit.

CMOS: Complementary metal-oxide semiconductor.

DAC: Digital-to-analog converter/conversion.

DSP: Digital signal processing.

Gm: Transconductor, a two-port voltage-to-current converter.

Gyrator: A two-port element that converts its load, $Z_{\mathrm{L}}$, into $1 / Z_{\mathrm{L}}$ at its input port.

IC: Integrated circuit.

IF: Intermediate frequency.

LAN: Local area network.

LNA: Low-noise amplifier.

Opamp: Operational amplifier.

OTA: Operational transconductance amplifier.

PLL: Phase lock loop.

RF: Radio frequency.

TIA: Trans-impedance amplifier.

VCO: Voltage-controlled oscillator. 


\section{CHAPTER 1}

\section{INTRODUCTION}

\subsection{The Need for RF Analog Filters and Oscillators}

In the last two decades or so, the number of Radio-Frequency (RF) communication integrated circuits (ICs) has seen a dramatic increase, for example, mobile phones, wireless computer peripherals such as cordless mice, wireless routers, inventory information networks, and hobbies. RF communication ICs extensively use oscillators and filters as local oscillators, intermediate frequency (IF) filters, channel-selecting filters and anti-aliasing filters, which span frequencies from about $200 \mathrm{kHz}$ to approximately $5.2 \mathrm{GHz}$, depending on their location within the radio [1]. Though functionally distinct, the design and theory of filters and oscillators are closely related.

Digital circuits also use oscillators and sometimes filters. As the clock speed reaches several GHz, microprocessors and Application Specific Integrated Circuits (ASICs) are seeing increased use of RF oscillators for clock generation and timing recovery [2]. Filters are widely used in phase locked loops (PLLs) and signal equalization. To be competitive, the ICs must be low-cost. When in mass production, the costs of filters and oscillators are primarily determined by their die area. Thus, reducing die area is a major objective of this work.

Signal filtering and generation can also be done by Digital Signal Processing (DSP) techniques, with high predictability. However, they are generally limited to signal frequencies below $500 \mathrm{MHz}$. Between $500 \mathrm{MHz}$ and $1 \mathrm{GHz}$, there are serious tradeoffs between analog processing and DSP in terms of power and cost. To 
implement real-time DSP above $1 \mathrm{GHz}$, the sampling rate and computing circuitry must operate at $10-100 \mathrm{GHz}$, which is not yet possible, not to mention the tremendous cost due to circuit complexity, silicon area and power dissipation. On the other hand, a properly designed analog circuit can readily process gigahertz signals with moderate power and costs.

The discussion in this work is limited to continuous-time circuits, as some filters, such as switched-capacitor filters, are analog, but not continuous-time. However, "analog" will be used throughout this work for brevity. In the general sense, "radiofrequency" includes all frequencies at which electromagnetic signals can be transmitted, either by line-of-sight propagation and reflections or guided by ionosphere and terrestrial surface, and that starts from approximately $3 \mathrm{kHz}[3]$. In this work, by "radio frequency", we imply frequencies above $1 \mathrm{GHz}$, which is also roughly the starting frequency of "microwave" circuits. However, microwave circuits are treated as distributed circuits, while the circuits in this work are designed so that even though they operate at microwave frequency, the distributed effects are negligible and the circuits can be treated as lumped for design and simulation purposes.

\subsection{Example Applications}

RF oscillators and filters found their first and most popular applications in the wireless communication industry, which includes mobile phones and wireless local connections such as Local Area Networks (LANs) and computer peripherals, understandably, due to the high-frequency requirements. In many cases the baseband signal processing is done by DSP, because of its predicable performance using digital 
CMOS process; however, signals above the IF frequency have to be processed by analog RF circuitry. The RF front-end and baseband are interfaced by analog-todigital converters (ADCs) and digital-to-analog converters (DACs).

Shown in Fig. 1-1 is the architecture of a typical wireless communication IC [1]: the antenna and its impedance matching network / band-selecting filter are shared between inbound $(\mathrm{Rx})$ and outbound (Tx) signals by means of an RF switch. Two mixers implement down conversion and up conversion for the Rx and Tx signals, respectively. An RF oscillator phase-locked to a reference frequency from an offchip crystal oscillator provides the signal for the mixers.

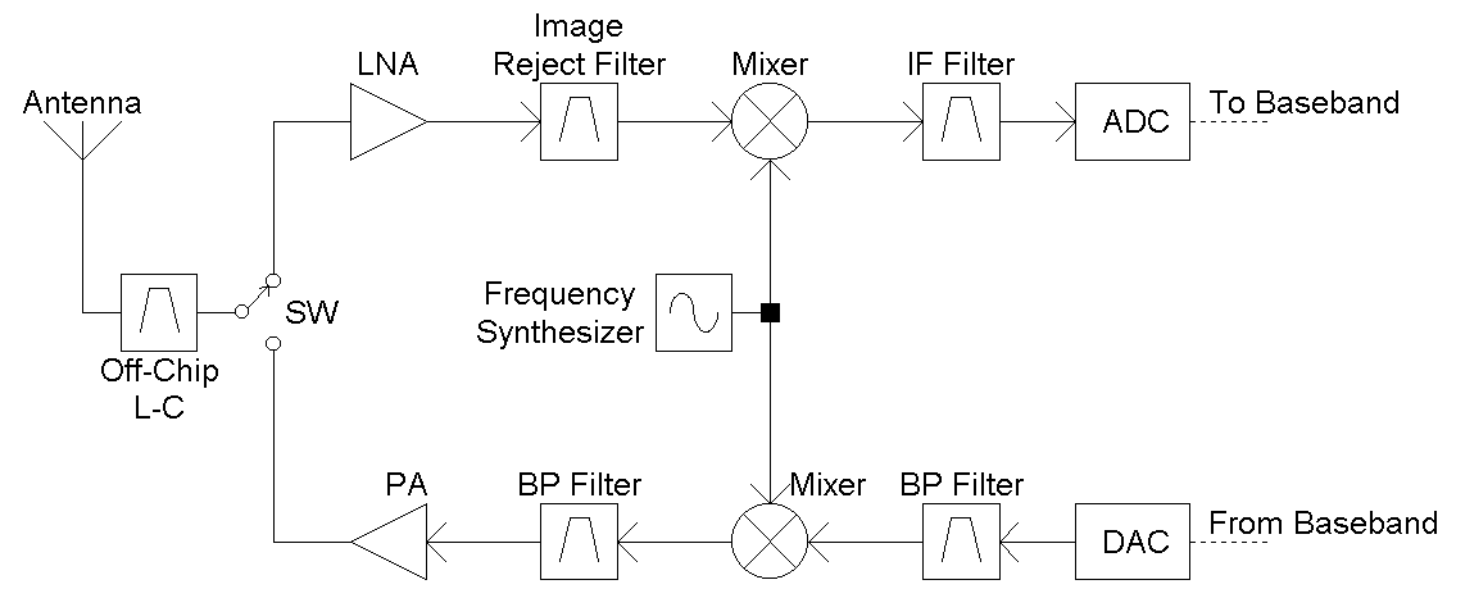

Figure 1-1 The architecture of a wireless communication IC.

Low-to-medium frequency analog filters are needed for IF filtering, ADC antialiasing and DAC waveform smoothing. Gigahertz bandpass filters are needed for the band selection, image rejection and Tx spectrum shaping. The band-selection filter rejects out-of-band large interferers and prevents them from saturating the LNA. The image-rejection filter prevents the image frequency from showing up in the downconverted signal band and interfering with the desired signals. The image rejection 
filter can be omitted if polyphase (in-phase and quadrature) signal processing is used to the right of the mixers, but that entails quadrature local oscillator and mixers, and doubles the silicon area and power dissipation [1]. The Tx spectrum shaping filter prevents the out-of-band signals from being transmitted, as required by industry regulations.

Gigahertz oscillators are also used in high-speed digital circuits, such as Phase Locked Loops (PLLs), as shown in Fig. 1-2. Different from wireless communication ICs, which require low harmonic distortion sinusoidal signals in the RF section, the output signals in digital circuits are ideally pulses. However, when operated at frequencies above $500 \mathrm{MHz}$, the signal path bandwidth becomes low compared with the frequency, the signal edges are rounded, and analog techniques must be used.

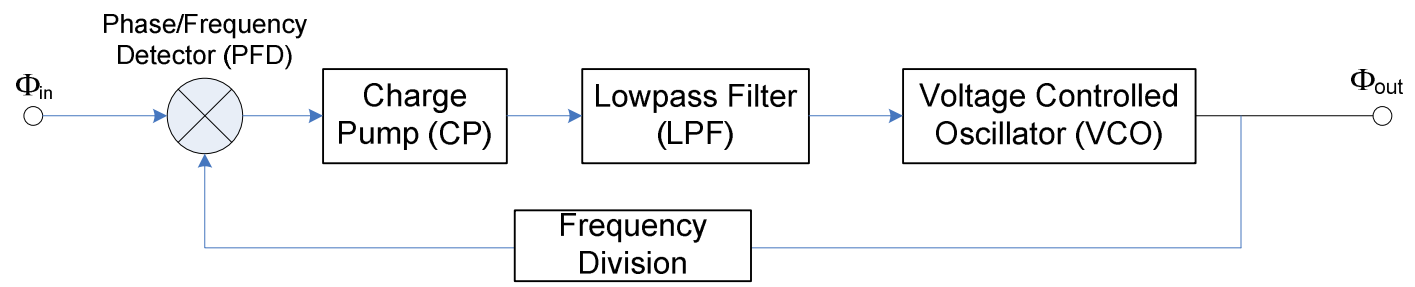

Figure 1-2 Block diagram of a charge-pump PLL.

Inside the PLL, the lowpass loop filter usually operates at a few MHz, while the VCO can be as high as a few $\mathrm{GHz}$.

\subsection{Implementation Methods and Literature Survey}

The implementation methods of analog filters and oscillators can be categorized into active and ( $Q$-enhanced) passive, depending on whether any passive elements such as inductors are involved. In most cases "passive designs" have active circuitry and consumes power in order to enhance the circuit $Q$ or realize oscillation, hence they are 
more accurately called enhanced passive designs. We classify a circuit as active if only transistors and capacitors are used. The implementation method also depends largely on the operating frequency, because basic electricity laws determine the value of circuit components and there are severe technical limitations on available integrated components, no matter how sophisticated or advanced the fabrication process is. To be sure, transistors are plentiful and cheap (but their power dissipation can be expensive); large capacitors and resistors are expensive and may not be available at all; inductors are expensive and may not be available, and large inductance values $(>\sim 100 \mathrm{nH})$ are simply impossible.

Active circuits can be used to design oscillators and filters at almost any frequencies, with varied performance and tradeoffs. Below $\sim 100 \mathrm{kHz}$, filters and oscillators are best designed using opamp- $R$ - $C$ circuits, likely with a few off-chip capacitors [4]. Between $100 \mathrm{kHz}$ and $\sim 100 \mathrm{MHz}$, transconductance- $\mathrm{C}\left(G_{\mathrm{M}^{-}} C\right)$ is the most popular method and can be fully integrated. The transconductors typically use linearization techniques to improve dynamic range, though the linearization circuits create extra noise. Between $100 \mathrm{MHz}$ and $\sim 500 \mathrm{MHz}, G_{\mathrm{M}^{-}} C$ method can still be used, but the requirement on $G_{\mathrm{M}}$ is much higher: they must have minimum parasitic capacitance and phase shift. For frequencies above $500 \mathrm{MHz}$, the $G_{\mathrm{M}} \mathrm{s}$ are usually reduced to single-transistor or minimum-transistor-count circuits, and the capacitors are very small or completely eliminated, with parasitic capacitance being used to obtain the desired operating frequency.

If passive inductors are available, they can usually be used to design oscillators and filters operating from $500 \mathrm{MHz}$ to $7-10 \mathrm{GHz}$. For operating frequencies below 500 
$\mathrm{MHz}$, the required inductance value is too large and difficult to implement. On the other hand, the self-resonance frequencies of passive inductors are usually around 6-7 $\mathrm{GHz}$, and thus they cannot be used to obtain operating frequencies above that either. Passive inductors have low $Q$ s between 5 and $\sim 25$, typically around 10. For oscillators, the low $Q$ (and other circuit losses) is compensated by a negative resistance circuit, and the inductor loss only contributes to the oscillator output noise, typically measured in terms of phase noise. To implement narrow-band or high-order lowpass filters, the low $Q$ will have to be "enhanced" by negative resistors [5] or some feedback scheme such as the use of integrated transformers [6].

Oscillators and filters can also be designed using microwave methods, for operating frequencies from $\sim 500 \mathrm{MHz}$ to above $10 \mathrm{GHz}$. Generally, transmission lines built from metal interconnects are involved. There are two main ways of using transmission lines. Transmission lines of a certain length can generate a specific phase shift at a certain frequency. When used with active circuits in feedback or feedforward, oscillators and filters can be obtained. Alternatively, terminated (open or ac short-circuit) transmission line stubs can have an inductive or capacitive input impedance of a certain value around a certain frequency, and be used in building oscillators and filters. Note that the transmission line stubs are equivalent to an inductor or a capacitor of desired value only near the design frequency. Therefore, they can only be used to build filters operating over a relative narrow bandwidth. Away from the design frequency, the inductor or capacitor equivalence no longer holds. Computer optimization methods can be used to expand the frequency band of filters designed this way [7]. 
Finally, oscillators and filters can be implemented using off-chip passive elements, such as crystal and Surface Acoustic Wave (SAW) devices. They utilize some kind of mechanical resonance, and by converting back and forth mechanical movements with electrical signals, implement electrical filters and oscillators. Electrically, the device is equivalent to an $R-L-C$ resonator tank, but with extremely high $Q$ s up to a few thousands. However, they generally cannot be fabricated on silicon dies or packaged with a die on the same IC, hence, they are expensive to use.

This work focuses on the implementation of oscillators and filters operating above $1 \mathrm{GHz}$, using transistors and capacitors only.

\subsection{Technical Requirements Summary}

The targets of this research are:

1. Integrated oscillators and high- $Q$ bandpass filters operating at the highest possible frequency as limited by the available technology.

2. Fabricated in the latest deep-submicron standard digital CMOS technology.

3. Operating at $1.8 \mathrm{~V}$ or lower.

4. Only transistors and capacitors compatible with digital CMOS are to be used.

5. The circuits are to be designed using minimum device sizes.

6. The circuits need to be robust. No trimming is allowed to overcome process variations, and the high- $Q$ filters must be stable when biased with regular commercial components.

7. The oscillators and filters must be electronically tunable to allow for correction of errors from modeling inaccuracies, process variations and environmental 
disturbances. The oscillators and filters must provide the appropriate tuning handles for automatic tuning.

Once again, the discussion hereafter is limited to lumped integrated circuits composed primarily of transistors and capacitors, with occasional involvement of passive inductors. Admittedly, above $1 \mathrm{GHz}$, analog ICs demonstrate distributed effects at times. However, attention has been paid in the circuit design and layout to minimize the distributed effects, for example, by making the layout compact compared with the signal wavelength, always using voltage signals (low $R_{\mathrm{S}}$, high $R_{\mathrm{L}}$ ) or current signals, and increasing the equivalent $Z_{0}$ of long signal interconnects by making its inductance per unit length far greater than capacitance per unit length. Without this qualification, some of the statements in this work will not be correct in the strictest sense. 


\section{CHAPTER 2 \\ DESIGN OF RF ACTIVE INDUCTOR}

\subsection{The Need for RF Active Inductor}

Inductors $(L)$, either passive or simulated using active circuitry, are needed for implementing filters and oscillators. Admittedly, using opamp and $R-C$ phase shift networks, oscillators such as the Wien-Bridge circuit may be implemented and lowquality-factor (low- $Q$ ) filters may be obtained using only $R$ and $C$ [4], but these cases are in the minority and cannot meet the performance requirement of many, if not most, applications. Equivalent (i.e., active) inductors are not always easily detected in active $R-C$ circuits, especially when the circuit is designed or explained using signal flow, state variables, or other methodologies rather than starting from a passive $L-C$ prototype. Nonetheless, many oscillators and filters designed using other concepts, such as integrator loops or recursive filtering [8], can almost always be explained by demarcating the circuit elements into active inductor(s), in part, because most filters and oscillators can be equated to prototype $R-L-C$ networks, where the $L$ is implemented actively.

To be useful, the active inductor has to have a self-resonance frequency higher than that of the oscillators and filters, and with some margin, because accessory circuits have additional parasitic capacitance, which will lower the operating frequency.

The requirement on active inductor $Q$ varies among applications. The $Q$, in

principle, does not have to be high in designing oscillators. However, a low- $Q$ active 
inductor will require a higher-gain feedback circuit, and increase power dissipation and circuit noise. Hence, we are motivated to design a high- $Q$ active inductor for oscillators without introducing undue noise or power dissipation. High- $Q$ bandpass filters and high-order narrow-transition-band lowpass filters also require high- $Q$ inductors.

Low-order lowpass filters do not require high- $Q$ inductors. However, a high- $Q$ inductor will reduce performance errors, such as dc-gain errors, and generally make the design process easier simply because the inductor is closer to an ideal inductor.

\subsection{Active-Inductor Design Method}

\subsubsection{The Gyrator Approach}

All active inductors must have at least one capacitor whose voltage lags its current by $90^{\circ}$ at ac steady state. Through a properly arranged active circuit, the voltage at the input port of the active inductor can be made to lead the input current by $90^{\circ}$. Thus, an inductive input impedance or an equivalent inductor is implemented. Note that the I-V relationship at ac in transistors (excluding their parasitic capacitance) and resistors is always $0^{\circ}$ or $180^{\circ}$. Hence, a pure transistor and/or resistor circuit without any capacitor can never generate a $90^{\circ} \mathrm{I}-\mathrm{V}$ relationship to implement an active inductor.

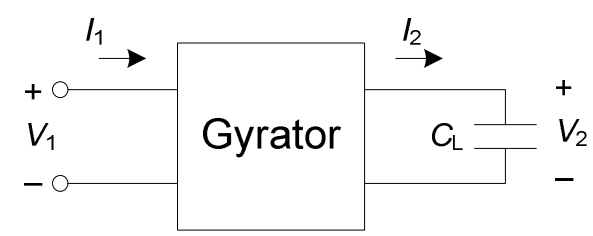

Figure 2-1 Implementing an active inductor using a gyrator.

The conversion is shown in Fig. 2-1. The black box that does the I-V 
phase/magnitude translation on the $C_{\text {Load }}$ is called a "gyrator", because it inverses the load impedance:

$$
Z_{\text {in }}=\frac{R^{2}}{Z_{\mathrm{L}}}=R^{2} s C_{\mathrm{L}}=s L_{\mathrm{eq}}
$$

where $L_{\mathrm{eq}}=R^{2} C_{\mathrm{L}}$ is the equivalent inductance.

The gyrator was proposed by Bernard D. H. Tellegen around 1948 [9], though it is possible that other researchers were working on the same circuit behavior earlier but did not conceptualize and publish it. All active inductor circuits can be analyzed using basic circuit analysis or other methods; but the use of the gyrator concept makes the analysis more concise.

\subsubsection{Active Inductor Implementation Methods}

Electronic gyrators, and in turn active inductors, can be built with opamps, Operational Transconductance Amplifiers (OTAs), or transistors. The building method largely depends on the desired self-resonance frequency $f_{\mathrm{R}}$ of the finished active inductor, since the active inductor will behave as an inductor only at frequencies (well) below $f_{\mathrm{R}}$. An opamp generally can be used to build active inductors with $f_{\mathrm{R}} \mathrm{S} \leq$ $1-5 \%$ that of its unity-gain frequency $f_{\mathrm{u}}$. For example, an LM741 has an $f_{\mathrm{u}}$ of $1.5 \mathrm{MHz}$, and can be used to build active inductors operating up to about $50 \mathrm{kHz}$. With frequency and $Q$ pre-distortion [10], we can build active-inductor filters operating slightly above this limit, but the design tradeoffs become severe rapidly. Since the maximum $f_{\mathrm{u}}$ of onchip CMOS opamps is about $1 \mathrm{GHz}$, we could expect to obtain opamp active inductors operating between 10-50 MHz. 


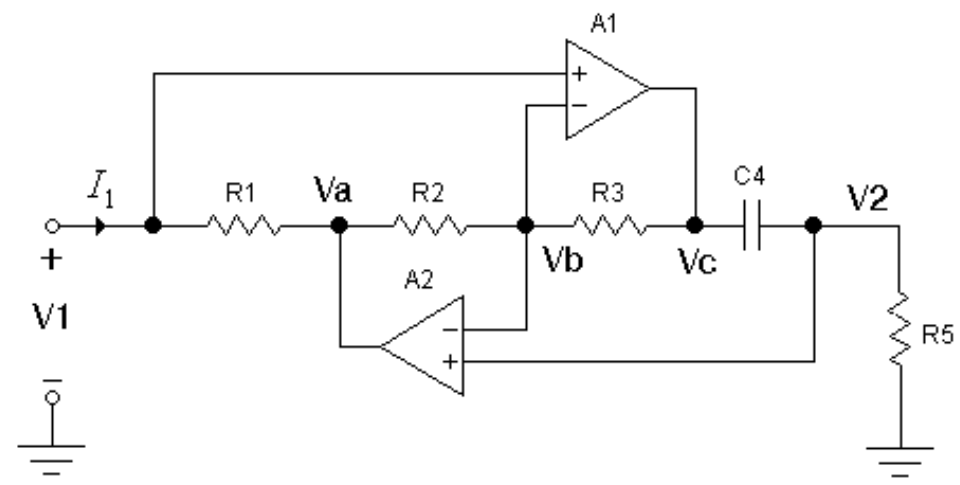

Figure 2-2 Implementing an active inductor using two opamps.

Shown in Fig. 2-2 is an example of implementing an active inductor with opamps. The input impedance is

$$
Z_{\text {in }}(s)=\frac{V_{1}}{I_{1}}=s C_{4} \frac{R_{1} R_{3} R_{5}}{R_{2}}=s L_{\mathrm{eq}}
$$

and,

$$
L_{\text {eq }}=C_{4} \frac{R_{1} R_{3} R_{5}}{R_{2}}
$$

The circuit may not appear to be a gyrator since it is seemingly loaded with a resistor $R_{5}$. But if we extract $C_{4}$ out of the circuit, and treat $V_{1}$-Gnd as Port 1 and $V_{\mathrm{C}^{-}} V_{2}$ as Port 2, then between Port 1 and Port 2 the circuit is a gyrator. A drawback of this circuit is that it requires a floating capacitor, which has a sizeable parasitic capacitance on its bottom plate in integrated implementations and will distort the circuit performance.

The phase shifts of the opamps will generate parasitic poles and zeros in $Z_{\text {in }}(s)$ and make the equivalent inductor non-ideal [10]. Nonetheless, at moderate frequencies the circuit is equivalent to an inductor to the first order. 
Above $10 \mathrm{MHz}$, the appropriate way of building active inductors is OTA-C, or rather, transconductor- $C\left(G_{\mathrm{M}^{-}} C\right)$ method. The difference between OTA and $G_{\mathrm{M}}$ is obscure. Common understanding is that OTA typically means a transconductor with a differential-pair input, and in most cases, optimized for high $R_{\mathrm{OUT}}$ and high dc gain; while $G_{\mathrm{M}}$ is a transconductor with only one or a few transistors, with a particular transconductance value and higher linearity than OTA. This work concentrates on $G_{\mathrm{M}^{-}}$ $C$ implementation, though the underlying principles largely the same.

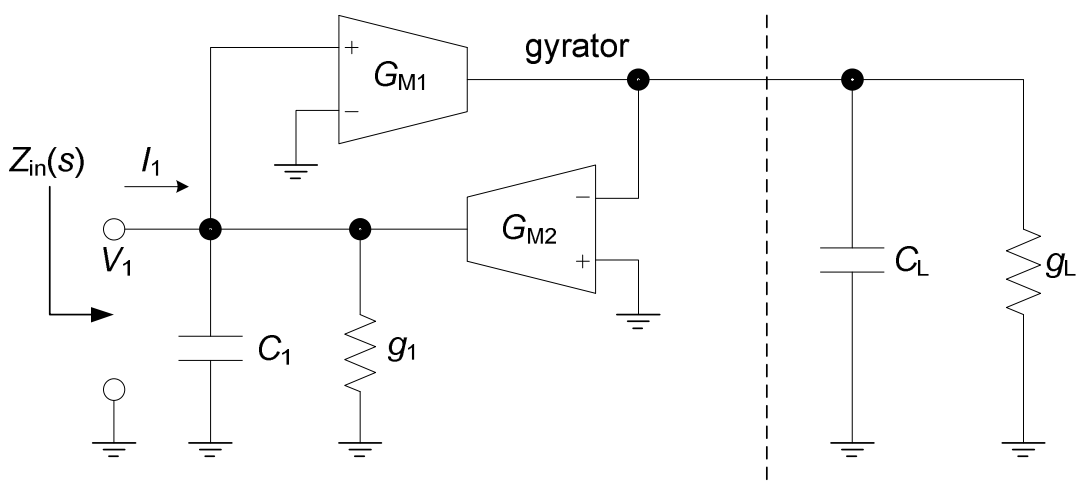

Figure 2-3 Implementing an active inductor using OTAs or $G_{\mathrm{M}} \mathrm{s}$.

As shown in Fig. 2-3, by connecting an inverting and a noninverting transconductor in a negative feedback loop, a gyrator is derived. When loaded with a capacitor, the input impedance of the gyrator is that of an equivalent inductor. Ignoring $C_{1}, g_{1}$ and $g_{\mathrm{L}}$ for the moment, using simple circuit analysis methods, the input impedance is calculated to be

$$
Z_{\text {in }}(s)=\frac{V_{1}}{I_{1}}=s \frac{C_{\mathrm{L}}}{G_{\mathrm{M} 1} G_{\mathrm{M} 2}}=s L_{\mathrm{eq}}
$$

which is an equivalent ideal inductor. Practical $G_{\mathrm{M}}$ cells have non-zero input and output capacitance, $C_{\mathrm{i}}$ and $C_{\mathrm{o}}$, as well as finite output resistance, $R_{\mathrm{o}}$, and these are lumped together and modeled as $C_{1}, g_{1}$ and $g_{\mathrm{L}}$ in Fig. 2-3. With these parasitic 
elements, the input admittance becomes:

$$
\begin{aligned}
& Y_{\text {in }}=\frac{1}{Z_{\text {in }}}=\frac{G_{\mathrm{M} 1} G_{\mathrm{M} 2}}{s C_{\mathrm{L}}+g_{\mathrm{L}}}+s C_{1}+g_{1}=\frac{1}{s \frac{C_{\mathrm{L}}}{G_{\mathrm{M} 1} G_{\mathrm{M} 2}}+\frac{g_{\mathrm{L}}}{G_{\mathrm{M} 1} G_{\mathrm{M} 2}}}+s C_{1}+g_{1} \\
& =\frac{1}{s L_{\mathrm{eq}}+r_{\mathrm{s}}}+s C_{1}+g_{1}=\frac{1}{s L_{\mathrm{eq}}+r_{\mathrm{s}}}+s C_{\mathrm{p}}+g_{\mathrm{p}}
\end{aligned}
$$

whose equivalent circuit is shown in Fig. 2-4. The equivalent components are

$$
L_{\mathrm{eq}}=\frac{C_{\mathrm{L}}}{G_{\mathrm{M} 1} G_{\mathrm{M} 2}}, \quad r_{\mathrm{s}}=\frac{g_{\mathrm{L}}}{G_{\mathrm{M} 1} G_{\mathrm{M} 2}}, \quad C_{\mathrm{p}}=C_{1}, \quad g_{\mathrm{p}}=g_{1}
$$

Incidentally, all real passive inductors have the same equivalent circuit that will be discussed more in the next section.

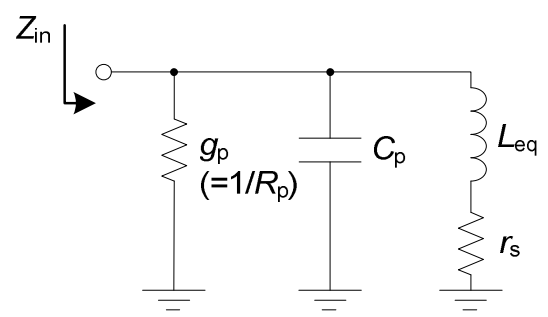

Figure 2-4 Equivalent circuit of a $G_{\mathrm{M}^{-}} C$ active inductor.

There are two issues limiting the operating frequency of $G_{\mathrm{M}^{-}} C$ active inductors:

(1) When the operating frequency increases, the parasitic capacitance and $G_{\mathrm{M}}$ value do not change substantially, thus the required $C_{\mathrm{L}}$ becomes smaller and smaller, and eventually negative at some point. It is true that equivalent negative capacitors can be implemented actively; however, in most cases, the active negative capacitor implemented using the same $G_{\mathrm{M}}$ cell will have a self-resonance frequency similar to that of the active inductor, hence it will not increase the self-resonance frequency of the entire circuit.

(2) The $G_{\mathrm{M}}$ cells have phase shift (usually a phase lag) that increases with 
frequency. A small phase shift $\left(<\sim 10^{\circ}\right)$ will generate an equivalent negative resistive element in $Z_{\text {in }}$, which increases the $Q$ of the inductor and will eventually render it unstable at a certain point. This will be discussed in detail in Section 2.5.

For operation above $500 \mathrm{MHz}$, the $G_{\mathrm{M}}$ cells in the active inductor circuit must be highly simplified, and highly efficient in terms of a high $G_{\mathrm{M}}$ to $C_{\mathrm{i}}, C_{\mathrm{o}}$ ratio. Linearization circuits will have to be eliminated to reduce the $G_{\mathrm{M}}$ phase lag. Singletransistor or minimum-transistor-count $G_{\mathrm{M}}$ cells have to be used. The load capacitor may be eliminated and only the transistor parasitic capacitance, such as $C_{\mathrm{gs}}$ and $C_{\mathrm{jd}}$, is used. There are severe tradeoffs among self-resonance frequency, linearity, noise, power dissipation and layout area.

\subsubsection{The Active-Inductor Equivalent Circuit}

Equation (2.5) can be further transformed into

$$
Z_{\text {in }}=\frac{\frac{1}{C_{\mathrm{p}}}\left(s+\frac{r_{\mathrm{s}}}{L_{\mathrm{cq}_{\mathrm{q}}}}\right)}{s^{2}+s\left(\frac{r_{\mathrm{s}}}{L_{\mathrm{eq}}}+\frac{g_{\mathrm{p}}}{C_{1}}\right)+\frac{1+g_{\mathrm{p}} r_{\mathrm{s}}}{L_{\mathrm{eq}} C_{\mathrm{p}}}}=\frac{\frac{1}{C_{\mathrm{p}}}\left(s+\omega_{\mathrm{z}}\right)}{s^{2}+s \frac{\omega_{\mathrm{R}}}{Q_{\mathrm{R}}}+\omega_{\mathrm{R}}^{2}}
$$

which is a lowpass function with possibly high peaking when $Q_{\mathrm{R}}$ is very high. The parameters are

$$
\begin{aligned}
& \omega_{\mathrm{z}}=\frac{r_{\mathrm{s}}}{L_{\mathrm{eq}}}, \omega_{\mathrm{R}}=\sqrt{\frac{1+g_{\mathrm{p}} r_{\mathrm{s}}}{L_{\mathrm{eq}} C_{\mathrm{p}}}} \approx \frac{1}{\sqrt{L_{\mathrm{eq}} C_{\mathrm{p}}}}, \\
& Q_{0}=\frac{\sqrt{\frac{1+g_{\mathrm{p}} r_{\mathrm{s}}}{L_{\mathrm{eq}}}}}{\frac{r_{\mathrm{s}}}{L_{\mathrm{eq}}}+\frac{g_{\mathrm{p}}}{C_{\mathrm{p}}}} \approx \frac{\sqrt{L_{\mathrm{eq}} C_{\mathrm{p}}}}{r_{\mathrm{s}} C_{\mathrm{p}}+g_{\mathrm{p}} L_{\mathrm{eq}}}=\frac{R_{\mathrm{p}} \sqrt{\frac{C_{\mathrm{p}}}{L_{\mathrm{eq}}}}}{1+\frac{r_{r_{\mathrm{p}}} \mathrm{C}_{\mathrm{p}}}{L_{\mathrm{eq}} g_{\mathrm{p}}}}=R_{\mathrm{p}} \sqrt{\frac{C_{\mathrm{p}}}{L_{\mathrm{eq}}}} \propto R_{\mathrm{p}}, \quad \text { when } r_{\mathrm{s}}=0
\end{aligned}
$$

The quality factor of the resonator, $Q_{\mathrm{R}}$, which is proportional to the shunt resistor $R_{\mathrm{p}}$, is also the quality factor of the $2^{\text {nd }}$-order denominator, and it is equal to $\omega_{\mathrm{R}}$ divided 
by the $-3-\mathrm{dB}$ bandwidth of the resulting bandpass filter [10]

$$
Q_{\mathrm{R}}=\frac{\omega_{\mathrm{R}}}{B W_{-3 \mathrm{~dB}}}
$$

The expression is exact only when $\omega_{\mathrm{z}}=0$, that is, the effect of $r_{\mathrm{s}}$ is ignored.

It shall be noted that $Q_{\mathrm{R}}$ is different from the inductor $Q$, which is a function of frequency and is defined as

$$
\begin{aligned}
& Q_{\mathrm{L}}(\omega)=\frac{\operatorname{Im}\left(Z_{\mathrm{in}}\right)}{\operatorname{Re}\left(Z_{\mathrm{in}}\right)}=\frac{\omega L_{\mathrm{eq}}}{r_{\mathrm{s}}} \frac{1-\frac{\omega_{\mathrm{z}}^{2}}{\omega_{\mathrm{R}}^{2}}-\frac{\omega^{2}}{\omega_{\mathrm{R}}^{2}}}{1+g_{\mathrm{p}} r_{\mathrm{s}}+\frac{\omega^{2}}{\omega_{\mathrm{R}}^{2}} \frac{g_{\mathrm{p}}}{C_{\mathrm{p}}} \frac{1}{\omega_{\mathrm{z}}}} \approx \frac{\omega L_{\mathrm{eq}}}{r_{\mathrm{s}}}, \\
& \text { when } \omega_{\mathrm{z}}<<\omega_{\mathrm{R}}, \omega<<\omega_{\mathrm{R}}, r_{\mathrm{s}}<<R_{\mathrm{p}} \text {, and } g_{\mathrm{p}} / C_{\mathrm{p}}<<\omega_{\mathrm{z}} .
\end{aligned}
$$

It should also be noted that

$$
Q_{\mathrm{L}}\left(\omega_{\mathrm{R}}\right)=\left.\frac{\omega L_{\mathrm{eq}}}{r_{\mathrm{s}}} \frac{-\frac{\omega_{z}^{2}}{\omega_{\mathrm{R}}^{2}}}{1+g_{\mathrm{p}} r_{\mathrm{s}}+\frac{g_{\mathrm{p}}}{C_{\mathrm{p}}} \frac{1}{\omega_{\mathrm{z}}}}\right|_{g_{\mathrm{p}}=0}=-\frac{r_{\mathrm{s}}}{\omega_{\mathrm{R}} L_{\mathrm{eq}}} \neq Q_{\mathrm{R}}
$$

$Q_{\mathrm{R}}$ is mainly determined by $R_{\mathrm{p}}$, when $r_{\mathrm{s}}$ is relatively small; and $Q_{\mathrm{L}}$ is primarily determined by $r_{\mathrm{s}}$. To increase $Q_{\mathrm{L}}, r_{\mathrm{s}}$ should be decreased, and this requires the reduction of load leakage $g_{\mathrm{L}}$ per Eq. (2.6). Applying Eq. (2.6) on Eq. (2.8), the expression for $Q_{\mathrm{R}}$ becomes

$$
Q_{\mathrm{R}}=\frac{\sqrt{G_{\mathrm{M} 1} G_{\mathrm{M} 2} C_{\mathrm{L}} C_{1}}}{g_{\mathrm{L}} C_{1}+g_{1} C_{\mathrm{L}}}=\frac{\sqrt{G_{\mathrm{M} 1} G_{\mathrm{M} 2} C_{\mathrm{L}} C_{\mathrm{p}}}}{g_{\mathrm{L}} C_{\mathrm{p}}+g_{\mathrm{p}} C_{\mathrm{L}}}
$$

When the highest operating frequency is desired and the capacitors are all parasitic capacitors, we have $g_{\mathrm{L}} \approx g_{1}$ and $C_{1} \approx C_{\mathrm{L}}$, since they have similar origins. Thus $g_{1}$ and $g_{\mathrm{L}}$ have similar contributions to $Q_{\mathrm{R}}$, and increasing $Q_{\mathrm{R}}$ becomes a collective effort of reducing both $g_{1}$ and $g_{\mathrm{L}}$, or some other measures may be used. 
As we shall see later, $g_{1}$ and $g_{\mathrm{L}}$ are not limited to the summation of transistor $g_{\mathrm{ds}} \mathrm{s}$; they can be equal to or greater than the values of the two primary transconductors $G_{\mathrm{M} 1}$ and $G_{\mathrm{M} 2}$ depending on the particular active-inductor circuit architecture. When $g_{1}$ is large, $R_{\mathrm{p}}$ and in turn $Q_{\mathrm{R}}$ will be low, and the inductor is not suitable for designing high- $Q$ bandpass filters. When $g_{\mathrm{L}}$ is large, $r_{\mathrm{s}}$ will be large, and the inductor cannot be used as a good inductor. Per Eq. (2.12), a large $g_{\mathrm{L}}$ will yield a low $Q_{\mathrm{R}}$, so it cannot be used as a high- $Q$ resonator either.

It should be noted that in many publications, when compensating for the circuit loss to obtain a high $Q$, it is not stated which loss resistor is targeted: $r_{\mathrm{s}}$ or $R_{\mathrm{p}}$, even though the compensation methods for them are usually very different. As a rule of thumb, active inductors used in digital (pulse) circuits, such as the input amplifier of fiber optic receivers or to implement wide-band amplifiers, are concerned primarily with $r_{\mathrm{s}}$; while active inductors used in bandpass and high-order lowpass filters are mainly concerned with $R_{\mathrm{p}}$. Obtaining high- $Q_{\mathrm{R}}$ circuit to implement narrow-band bandpass filters is one of the targets of this work.

The simulation results of an example are given in Fig. 2-5. Below $f_{\mathrm{z}}=32 \mathrm{MHz}$, $Z_{\text {in }}=10 \Omega$ is a resistor; between $f_{\mathrm{z}}$ and $f_{0}=2.25 \mathrm{GHz}, Z_{\text {in }}$ is equivalent to a $50 \mathrm{nH}$ inductor, as its magnitude is proportional to $\omega$; above $f_{0}, Z_{\text {in }}$ is equivalent to a $100 \mathrm{fF}$ capacitor, as its magnitude is inversely proportional to $\omega$. The $Q_{\mathrm{L}}$, defined by $\operatorname{Im}\left(Z_{\text {in }}\right) / \operatorname{Re}\left(Z_{\text {in }}\right)$, is negative when the admittance from $C_{\mathrm{p}}$ is greater than that from $L_{\mathrm{eq}}$. Low-frequency $Q$ is determined by $r_{\mathrm{s}}$, and high-frequency $Q$, in particular that at $f_{\mathrm{R}}$, is determined by $R_{\mathrm{p}}$. Beyond $f_{\mathrm{R}}$, the circuit is not of practical use, because it behaves as a 
capacitor, but is more complex and consumes power in the case of an active inductor.
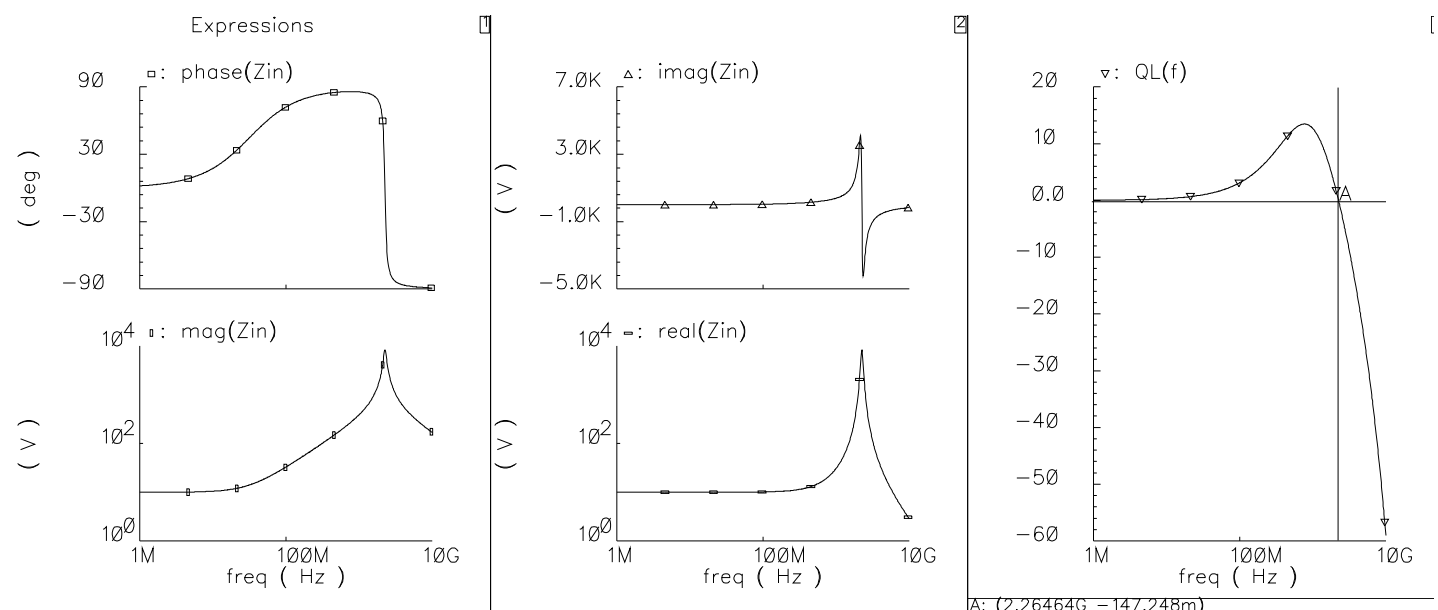

Figure 2-5 Simulation plots of the circuit in Fig. 2-4 with $R_{\mathrm{p}}=1 / g_{\mathrm{p}}=10 \mathrm{k} \Omega, C_{\mathrm{p}}=100 \mathrm{fF}, L_{\mathrm{eq}}=50 \mathrm{nH}$, $r_{\mathrm{s}}=10 \Omega$.

\subsection{Technology Constraints}

A semiconductor fabrication process is a collection of steps (masking, doping, etc.) and technical specifications (topological, electrical, chemical, etc.) that define a production, and consequently, a consortium of devices and interconnects of unique characteristics. Many companies have their own processes, with many more derivations to address different needs in different products, such as high-speed (e.g., data transceivers), low-power (e.g., microprocessors), high-frequency (e.g., RF and microwave ICs), high-density (e.g., memories). As a result, there are probably thousands of processes in the world. However, when categorized by their key masking steps and primary device (transistor) structures, the plethora of processes is reduced to a handful "technologies," with different characteristics.

Bipolar technology was the first technology to be used in the mass production of ICs. Its primary devices are NPN and PNP transistors, with resistors and capacitors 
available using extra steps. Bipolar is stable, mature, moderately fast, with good device properties for both digital and analog circuits. Its primary disadvantage is cost, because of its relatively low integration density and extra fabrication steps compared with CMOS (complementary metal oxide semiconductor). Therefore, its product share has been on constant decline over the last few decades, thought its absolute volume has probably been increasing, considering the exponential increase in semiconductor manufacturing. Bipolar Junction Transistors (BJTs) are necessary for implementing bandgap reference and other circuits, and hence are preserved on many CMOS processes. The base current of bipolar transistors is not zero, and this limits their use in memory and other low-power circuits.

CMOS technology is currently the mainstream of the semiconductor industry, mainly because of its low cost. It is the ideal technology for digital circuits: high integration density, nearly zero gate dc currents, and low leakage in the off state. Unfortunately, compared with bipolar technology, it is not the ideal technology for analog circuits because of its higher level of noise and nonlinearity, difficulty of accurate modeling, high threshold voltage $\left(V_{\mathrm{TH}}\right)$ mismatch, and some functional circuits, such as the bandgap reference, must use bipolar transistors. The long-channel $(L \geq 1 \mu \mathrm{m})$ devices are well modeled by the square-law $I-V$ characteristic, which can be used to build analog circuits with good linearity. However, short-channel devices deviate from the square-law significantly because of second-order effects, such as velocity saturation from the horizontal field and mobility degradation from the vertical field, and cannot be modeled accurately across the entire hyperspace of process corners, temperature, biasing voltages and currents, as well as device width and 
length. Intermediate-frequency $(<\sim 10 \mathrm{MHz})$ analog circuit design can dodge this problem by using long-channel devices in deep-submicron CMOS technologies; however, analog circuits operating above $1 \mathrm{GHz}$, as in this work, have to use minimum or near-minimum device length to reach the required frequency. Consequently, analog CMOS circuits operating above $1 \mathrm{GHz}$ have poor linearity, high noise level and wide performance variation.

Despite the drawbacks, a very sizeable portion of analog designs nowadays are done in CMOS and they have to reside in a hostile environment with devices optimized for digital signals and a high level of interference from digital circuitry being coupled over via the substrate and power supply. The reason is that on most ICs today, the area of digital circuits dominates the die area and determines the IC cost, so the technology selection is mainly based on the requirements of digital circuitry.

The primary devices in CMOS are NFETs and PFETs. Poly/N-Well resistors, metal-insulator-metal (MiM)/poly capacitors, MOS varactors, and passive inductors are available in some processes using extra masking steps. Most standard digital CMOS processes use an epitaxial substrate to reduce its resistivity $\rho$ and in turn the likelihood of latchup, and the only available devices are FETs. An example is the Taiwan Semiconductor Manufacturing Corp (TSMC)'s LOG018 process. Some CMOS processes are modified to accommodate the needs of analog and RF circuits, using non-epitaxial substrate to reduce RF substrate noise coupling; they are called “mixed-signal" process, such as TSMC's MM018 process, to the disadvantage of digital circuitry and at a cost premium. The MM018 process features a thick top metal for spiral inductors, capacitors and poly resistors. 
To compensate for the lack of BJTs, some CMOS processes are modified to provide substrate PNPs and/or triple-well NPNs along with FETs; they are referred to as BiCMOS technology. Compared with a pure bipolar technology, the performance of the BJTs is poorer; however, it is still better than MOSFETs for many analog designs. BiCMOS is on the decline and is being replaced by pure CMOS technology in many cases, where analog designs have to be accomplished using MOSFETs only.

Silicon-germanium (SiGe) BiCMOS is an expensive technology, but has seen an increase over the last decade, primarily due to the demand from the mobile phone industry. It combines high-performance heterojunction bipolar transistors (HBTs) with state-of-the-art CMOS technology. A SiGe base with a graded germanium doping profile is sandwiched between the Si collector and Si emitter of the HBT. A built-in electric field is created by making the Ge doping level higher toward the collector, and it accelerates electrons as they travel from the emitter to the collector across the base [11]. Therefore, the transition time of electrons is decreased and the HBTs can operate at a higher frequency than regular BJTs. Together with the availability of passive inductors and capacitors, the technology is suitable for high-performance RF ICs. The available MOSFETs make it possible to implement a small amount of digital circuitry on the same die with moderate costs.

Finally there is the Gallium-Arsenide (GaAs) technology that is primarily used for microwave ICs, though it is also used for very-high-speed digital circuits. By using the GaAs compound instead of silicon as substrate, a higher energy bandgap (1.4 V) and electron mobility $(\sim 6 \mathrm{x})$ are obtained compared with silicon, giving it the potential for high-temperature and very-high-speed applications [12]. The transistors in GaAs 
technology are metal-semiconductor field-effect-transistors (MESFETs). Instead of the silicon-oxide insulated gate in CMOS, METFET's gate is a Schottky diode whose depletion layer extends into the substrate at reverse biasing and pinch the conduction channel between source and drain. Similar to mixed-signal CMOS and BiCMOS, GaAs technology typically provides passive inductors and capacitors. Due to the highresistivity of the GaAs substrate, the passive inductors have higher $Q$ s than those on CMOS, and microwave structures, such as transmission lines, can be implemented onchip. These properties make GaAs more popular with RFICs designed with microwave methods.

To eliminate the need for more expensive technologies, this work is done using TSMC's LOG018 standard digital CMOS process. This will also make it possible to integrate RF active inductors directly with standard digital circuits without modifying the fabrication process. However, TSMC's LOG018 process, like most other modern standard digital CMOS processes, comes with a few severe constraints:

1. Only PFETs and NFETs are available, with relatively high threshold voltages that are engineered for digital circuitry to reduce power dissipation and leakage, but limit analog circuit topologies for a given power supply voltage $V_{\mathrm{DD}}$. This precludes the use of many proven linearization methods.

2. Poly capacitors are not available, so ac signal coupling is not possible, as done in many active inductors designed using microwave theory and in GaAs technologies. This requires that the circuit topologies are appropriate for both ac and dc, and restricts design possibilities. 
3. Interconnect capacitances are not accurately modeled since the primary application is digital circuits. Due to the high operating frequency, interconnect capacitance is a non-negligible part of circuit design, hence the fabricated circuits may be off the design target or may not function at all.

4. There is no specification on the maximum applicable frequency of the vendor device models. It is estimated to be below $1 \mathrm{GHz}$. The device modeling is not done using RF methods, which include gate and substrate resistance as well as other parasitic elements that are typically ignored in modeling for analog and digital circuits. The resulting circuit may act differently as simulated.

This work is to obtain operating circuits among the challenges.

\subsection{Literature Survey of Active Inductors}

In this section, transistor-level active inductors proposed by researchers over the years are reviewed. To expose their fundamental structure, biasing circuits and capacitor ac coupling (popular with microwave ICs, almost never in CMOS ICs) are not shown, but are pointed out when necessary. As we shall see below, all active inductors can be explained by a three-step operation:

1. Converting the port voltage $V_{\text {in }}$ into an in-phase $\left(0^{\circ}\right)$ current, more efficiently done by a transconductor, $G_{\mathrm{m}}$, and less efficiently by a resistor;

2. Applying this current on a capacitor and obtaining a voltage $V_{\mathrm{CL}}$ that is $-90^{\circ}$;

3. Converting $V_{\mathrm{CL}}$ into the input current $I_{\text {in }}$, almost always through a $G_{\mathrm{m}}$.

The reference directions have to be correct to yield a positive-value inductor with non-negative losses. 
Of course, an active inductor can be more complicated than this, e.g., involving more conversions between $V_{\text {in }}$ and $I_{\text {in }}$ or more parasitic poles in the conversions, but the circuit will be less efficient, and the advantages gained, such as linearity, are rarely worth the degradations in other aspects for $\mathrm{GHz}$ designs.

\subsubsection{Gate- $R$ and Gate- $R$-II Active Inductors}

The simplest active inductor is formed by placing a resistor at the gate of a MOSFET or the base of an NPN BJT, as shown in Fig. 2-6 (a) and (c). The circuit is usually called "active inductor" in the literature without a more specific name. For ease of discussion, we name it "gate- $R$," based on its structure.

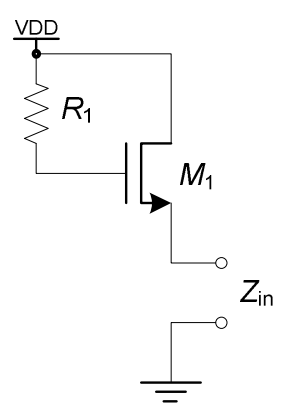

(a)

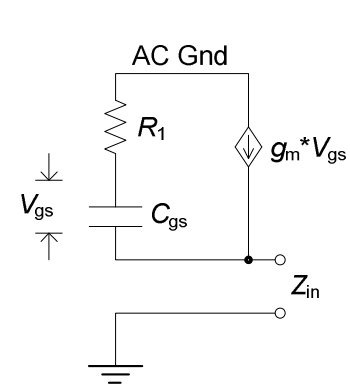

(b)

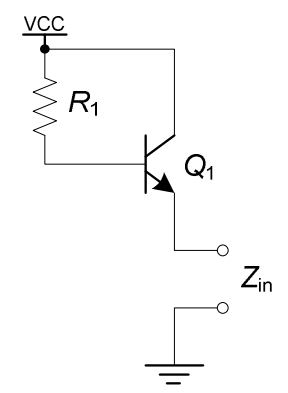

(c)

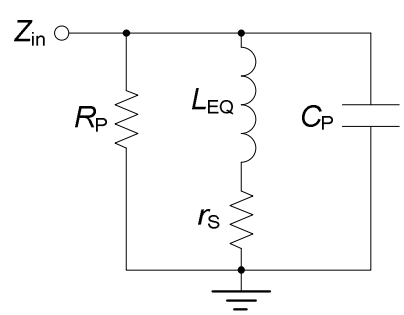

(d)

Figure 2-6 (a) CMOS gate- $R$ active inductor. (b) Small-signal ac equivalent circuit. (c) Bipolar version of the same circuit. (d) Equivalent circuit showing the inductor.

If $R_{1}$ is large compared with $\left|1 /\left(j \omega C_{\mathrm{gs}}\right)\right|$, the ac voltage $v_{\mathrm{gs}}$ can be assumed to be zero, and $R_{1}$ will convert $V_{\text {in }}$ into an in-phase current which is applied to the gatesource capacitor of $M_{1}, C_{\mathrm{gs}}$, the first step of inductor formation. For a MOSFET, using a small-signal equivalent circuit and ignoring parasitic components, the voltage across $C_{\text {gs }}$ is 


$$
V_{\mathrm{gs}}=V_{\text {in }} \frac{1 /\left(s C_{\mathrm{gs}}\right)}{R_{1}+1 /\left(s C_{\mathrm{gs}}\right)}=\left.V_{\text {in }} \frac{1}{s R_{1} C_{\mathrm{gs}}+1} \approx V_{\text {in }} \frac{1}{s R_{1} C_{\mathrm{gs}}}\right|_{w h e n|s|>>\frac{1}{R_{1} C_{\mathrm{gs}}}}
$$

If $R_{1}$ is large, we have $I_{\text {in }} \approx-I_{\mathrm{ds} 1}$. At relatively high frequencies, $V_{\mathrm{gs}}$, and in turn, $I_{\mathrm{in}}$, is going to $\operatorname{lag} V_{\text {in }}$ by $90^{\circ}$, and that is the property of an inductor. It can be easily verified that the reference directions of $V_{\text {in }}$ and $I_{\text {in }}$ are both correct.

If we remove the high-frequency condition and use small-signal analysis, the input impedance can be calculated as

$$
\begin{aligned}
Y_{\text {in }} & =\frac{g_{\mathrm{m}}+s C_{\mathrm{gs}}}{1+s R_{1} C_{\mathrm{gs}}}=\frac{g_{\mathrm{m}} G_{1}+s C_{\mathrm{gs}} G_{1}}{G_{1}+s C_{\mathrm{gs}}}=\frac{1}{R_{1}}+\frac{g_{\mathrm{m}} G_{1}-G_{1}^{2}}{G_{1}+s C_{\mathrm{gs}}} \approx \frac{1}{R_{1}}+\left.\frac{g_{\mathrm{m}} G_{1}}{G_{1}+s C_{\mathrm{gs}}}\right|_{G_{1}<<1} \\
& =\frac{1}{R_{1}}+\frac{1}{\frac{1}{g_{\mathrm{m}}}+\frac{s C_{\mathrm{gs}} R_{1}}{g_{\mathrm{m}}}}=\frac{1}{R_{\mathrm{p}}}+\frac{1}{r_{\mathrm{s}}+s L_{\mathrm{eq}}}
\end{aligned}
$$

where $g_{\mathrm{m}}$ is the transconductance of $M_{1}$ and $G_{1}=1 / R_{1}$. The circuit is equivalent to the one in Fig. 2-6(d), and the small-signal equivalent elements are

$$
R_{\mathrm{p}}=R_{1}, r_{\mathrm{s}}=\frac{1}{g_{\mathrm{m}}}, L_{\mathrm{eq}}=\frac{R_{1} C_{\mathrm{gs}}}{g_{\mathrm{m}}}
$$

Due to the parasitic capacitors, there inevitably is an equivalent shunt capacitor $C_{\mathrm{p}}$, which includes $C_{\text {js }}$ of $M_{1}$. The inductor $Q$ is

$$
Q_{\mathrm{L}}(\omega)=\frac{\left|j \omega L_{\mathrm{eq}}\right|}{r_{\mathrm{s}}}=\omega R_{1} C_{\mathrm{gs}}
$$

and the resonance $Q, Q_{\mathrm{R}}$, is jointly determined by $C_{\mathrm{p}}, R_{\mathrm{p}}$ and $L_{\mathrm{eq}}$.

Comparing this active inductor to the gyrator (Fig. 2-3), the $90^{\circ}$ phase shift from $V_{\text {in }}$ to $V_{\text {cap }}$ is obtained through a resistor, instead of a transconductor, and this conversion is not close to ideal (Eq. 2.13). This contributes to its very low $Q_{\mathrm{R}}$. 
This active inductor exists at the output of a source or emitter follower, causing undesirable signal peaking. However, it has been widely used in the Trans-Impedance Amplifier (TIA) and limiting amplifier of fiber optic receivers in the last two decades [13-15], for "shunt peaking" $[16,17]$, that is, improving the sharpness of pulse signal edges by partially "canceling out" the load capacitance. In fact, any wide-band pulsesignal processing circuits, such as amplifiers and mixers, can benefit from it for the added bandwidth. It is also used in oscillators [18-20], even though due to its very low $Q_{\mathrm{R}}$ (around 2) [19], the negative resistor has to be very large to make the oscillator work and hence consumes a high current.

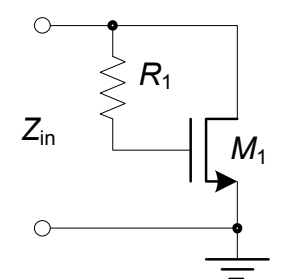

(a)

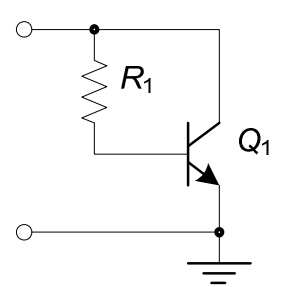

(b)

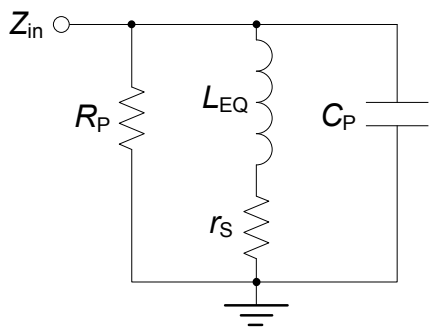

(c)

Figure 2-7 Gate- $R$-II active inductors and their small-signal equivalent circuit.

A slight connection variation in gate- $R$ active inductor results in another active inductor, as shown in Fig. 2-7, and is named "gate- $R$-II". For the MOSFET version, ignoring parasitic components and using the small-signal equivalent circuit, $Z_{\text {in }}$ is calculated as

$$
\begin{aligned}
Y_{\text {in }}= & \frac{s C_{\mathrm{gs}}+g_{\mathrm{m}}}{1+s R_{1} C_{\mathrm{gs}}}=\frac{1}{R_{1}}+\frac{1}{\frac{1}{g_{\mathrm{m}}-\frac{1}{R_{1}}}+s \frac{R_{1} C_{\mathrm{gs}}}{g_{\mathrm{m}}-\frac{1}{R_{1}}}} \approx \frac{1}{R_{1}}+\left.\frac{1}{\frac{1}{g_{\mathrm{m}}}+s \frac{R_{1} C_{\mathrm{gs}}}{g_{\mathrm{m}}}}\right|_{\text {when } g_{\mathrm{m}}>>1 / R_{1}} \\
= & \frac{1}{R_{\mathrm{p}}}+\frac{1}{r_{\mathrm{s}}+s L_{\mathrm{eq}}}
\end{aligned}
$$


where $C_{\mathrm{gs}}$ and $g_{\mathrm{m}}$ are the gate-source capacitance and transconductance of $M_{1}$, respectively, and the equivalent elements are

$$
R_{\mathrm{p}}=R_{1}, r_{\mathrm{s}}=\frac{1}{g_{\mathrm{m}}}, L_{\mathrm{eq}}=\frac{R_{1} C_{\mathrm{gs}}}{g_{\mathrm{m}}}
$$

$Q_{\mathrm{L}}(\omega)$ and $Q_{\mathrm{R}}$ have the same expression as that of the gate-R active inductor.

Interestingly, the gate-R-II and gate-R active inductors have identical input impedances. Nonetheless, $M_{1}$ in gate-R-II circuit is not subject to the body effect, which increases $V_{\text {th }}$ and affects the effective $g_{\mathrm{m}}$ of the transistor. The dc voltages at the input terminal of the two circuits are also different, one $V_{\mathrm{gs}, \mathrm{n}}$ down from $V_{\mathrm{DD}}$ and one $V_{\mathrm{gs}, \mathrm{n}}$ up from ground, respectively, and may fit different applications. Similar to the gate- $R$ active inductor, the gate- $R$-II also has very low $Q_{\mathrm{R}}$, which may or may not be an issue depending on the application.

The Gate-R-II circuit also found its most popular use in limiting amplifiers for optical receivers $[21,22]$. It has also been used in LNA design [23], in which BJTs were used to reduce the noise level.

In some works $[14,19,21,22]$, instead of a poly or diffusion resistor, the $R$ is implemented by a MOSFET operating in the linear region, as shown in Fig. 2-8.

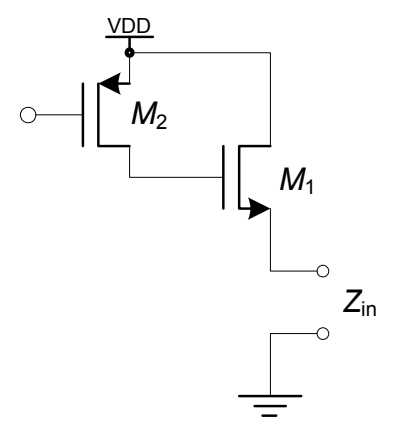

(a)

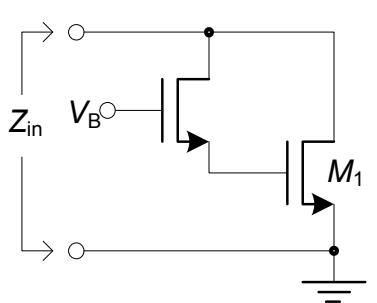

(b)

Figure 2-8 Gate- $R$ (a) and Gate- $R$-II (b) active inductors using a linear-region FET as gate resistor. 


\subsubsection{Cascode Gate- $R$ Active Inductors}

Real transistors have limited $R_{\text {out }}$, or non-zero $g_{\mathrm{ds}}$ in the case of CMOS, and it is in parallel with $R_{\mathrm{P}}$ in gate- $R$ and gate- $R$-II circuits, reducing $Q_{\mathrm{R}}$ and $Q_{\mathrm{L}}$. In an effort to increase $R_{\text {out }}$ and improve $Q$, cascode is introduced to $M_{1}$ in Fig. 2-7, resulting in the cascode gate- $R$-II circuit in Fig. 2-9(a).

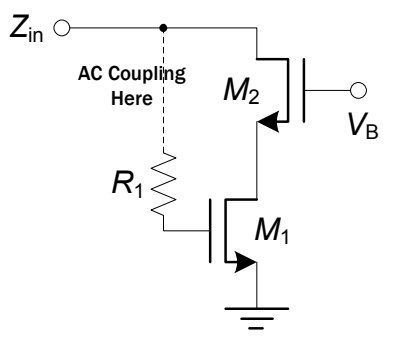

(a)

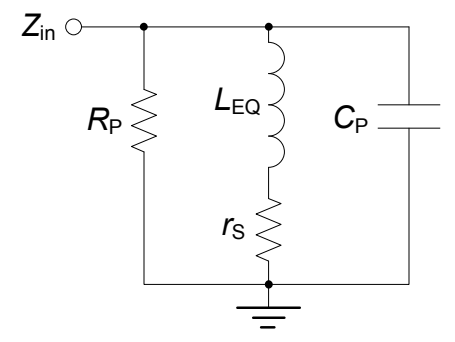

(b)

Figure 2-9 Cascode gate- $R$-II active inductor (Shinji Hara, 1988). (a) Primary circuit with ac coupling shown; (b) Equivalent circuit showing the inductor.

The circuit was proposed by Shinji Hara et al. in 1988 using an off-chip resistor $[24,25]$ and has seen many variations over the years, mostly in microwave circuits. Small-signal analysis yields [24, 25]

$$
L_{\mathrm{eq}}=\frac{R_{1} C_{\mathrm{gs}}}{g_{\mathrm{m}}}, r_{\mathrm{s}}=\frac{1}{g_{\mathrm{m}}}, C_{\mathrm{P}} \approx\left(\frac{\omega C_{\mathrm{gs}}}{g_{\mathrm{m}}}\right)^{2} C_{\mathrm{gs}}
$$

The improvement in $Q_{\mathrm{L}}$ is paltry, because $r_{\mathrm{s}}$ does not change. $Q_{\mathrm{R}}$, which is determined mainly by $R_{\mathrm{P}}$, can be improved, however, not due to the improvement of transistor $R_{\text {out }}$ from the use of cascode, as believed by many researchers, but from the $C_{\mathrm{gs}}$ of the cascode FET $M_{2}[26,27]$. The $C_{\mathrm{gs}}$ of $M_{2}$ introduces a parasitic pole to the circuit at its source, and this generates a phase lag in the gyrator loop. As we shall examine more closely in Section 2.5.3, a phase lag within the gyrator loop has the effect of improving $Q_{\mathrm{R}}$, and can even render the circuit unstable. 
Other efforts in compensating the loss and improving $Q$ include replacing the $R$ on the gate with another gate- $R$ active inductor (recursive topology) [28-30], as shown in Fig. 2-10.

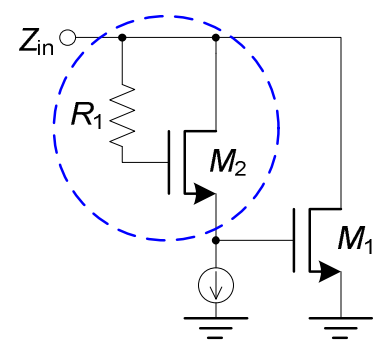

Figure 2-10 Recursive Gate- $R$-II active inductor.

The cascode gate- $R$ active inductors were used in optical receivers for wide-band pulse amplification [31], but also found use in RF filters [32-35] due to their potential for higher $Q_{\mathrm{R}}$, through the use of cascode and, more commonly, additional losscompensating circuitry.

\subsubsection{CG-CS Active Inductors}

As mentioned, many gate- $R$ active inductors are implemented by replacing the gate resistor with a linear-region MOSFET [14, 19, 21, 22], especially in optical receivers, where the prevalent technology is CMOS and usually does not allow resistors and large-value capacitors (for ac coupling) on chip. Another active inductor can be obtained by making the same gate-FET operating in the forward-active region in common-gate (CG) mode, as shown by Fig. 2-11, with a structure similar to that of the cascode gate- $R$-II circuit. Note that if only signal-path transistors are shown, it is not possible to tell whether the gate-FET $M_{2}$ is acting as a resistor or a CG transistor, and unfortunately, some papers do not include this information directly. One way to tell 
the operating region of the gate-FET is to observe the biasing. If there is a biasing current source connecting to the $M_{1}$ gate, $M_{2}$ is likely to be operating in CG mode; otherwise it has to be a resistor, because it has no dc biasing current path.

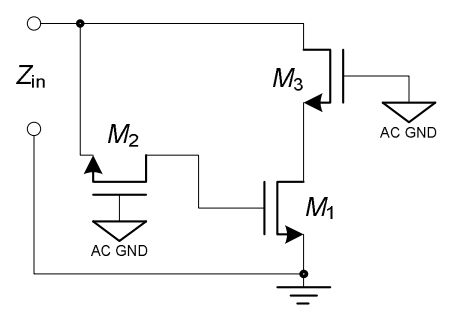

(a)

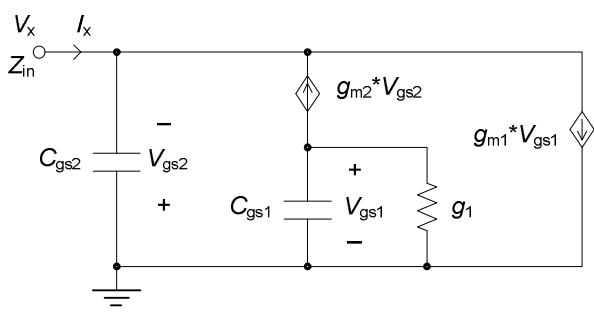

(b)

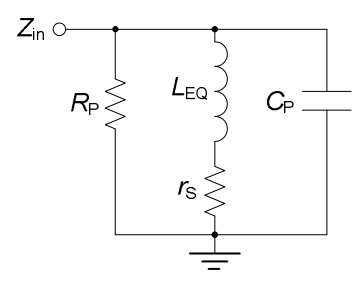

(c)

Figure 2-11 (a) CG-CS active inductor (Shinji Hara, 1989). (b) Small-signal ac circuit. $g_{1}$ is mainly from the $g_{\mathrm{ds}}$ of the biasing transistor (not shown) for $M_{2}$. (c) Equivalent circuit.

Shinji Hara et al. began their active inductor research $[24,25]$ with the gate- $R$ circuit and then replaced the external resistor with a common-gate MOSFET $M_{2}$ [36], as shown in Fig. 2-11. This circuit is named "CG-CS" active inductor because the two transistors along the signal path operate in common-gate $\left(M_{2}\right)$ and common-source $\left(M_{1}\right)$, respectively. The original motive was to eliminate the off-chip resistor in the gate- $R$ cirucit and fully integrate it; however, this circuit is very remotely related to the gate- $R$ active inductor.

$M_{2}$ is a noninverting $G_{\mathrm{M}}$ between $V_{\mathrm{in}}$ and $C_{\mathrm{gs}}$, and $M_{1,3}$ forms an inverting $G_{\mathrm{M}}$ from $C_{\mathrm{gs} 1}$ back to $V_{\mathrm{in}}$. Note that $M_{3}$ is optional and its main function is to increase the resistance looking into the $M_{1}$ drain. Using the small-signal ac equivalent circuit in Fig. 2-11(b), the circuit input impedance is calculated as

$$
Y_{\mathrm{in}}=g_{\mathrm{m} 2}+s C_{\mathrm{gs} 2}+\frac{g_{\mathrm{m} 1} g_{\mathrm{m} 2}}{s C_{\mathrm{gs} 1}+g_{1}}=g_{\mathrm{m} 2}+s C_{\mathrm{gs} 2}+\frac{1}{s \frac{C_{\mathrm{gs} 1}}{g_{\mathrm{m} 1} g_{\mathrm{m} 2}}+\frac{g_{1}}{g_{\mathrm{m} 1} g_{\mathrm{m} 2}}}
$$

which can be represented by the equivalent circuit in Fig. 2-11(c), with elements being 


$$
R_{\mathrm{p}}=\frac{1}{g_{\mathrm{m} 2}}, C_{\mathrm{p}}=C_{\mathrm{gs} 2}, L_{\mathrm{eq}}=\frac{C_{\mathrm{gs} 2}}{g_{\mathrm{m} 1} g_{\mathrm{m} 2}}, r_{\mathrm{s}}=\frac{g_{1}}{g_{\mathrm{m} 1} g_{\mathrm{m} 2}}
$$

The quality factors are

$$
Q_{\mathrm{R}}=R_{\mathrm{p}} \sqrt{\frac{C_{\mathrm{p}}}{L_{\mathrm{eq}}}}=\sqrt{\frac{g_{\mathrm{m} 1}}{g_{\mathrm{m} 2}}}, Q_{\mathrm{L}}(\omega)=\frac{\omega L_{\mathrm{eq}}}{r_{\mathrm{s}}}=\frac{\omega C_{\mathrm{gs} 2}}{g_{1}}
$$

The circuit is a very-low- $Q_{\mathrm{R}}\left(\approx 1\right.$ if $\left.g_{\mathrm{m} 1} \approx g_{\mathrm{m} 2}\right)$ active inductor due to $R_{\mathrm{p}}=1 / g_{\mathrm{m} 2}$. Another way to look at this is that the source of $M_{2}$ is connected to the inductor port and the resistance looking into the $M_{2}$ source is $1 / g_{\mathrm{m} 2}$, a small number compared with other equivalent elements. Therefore, the claim of "lossless" for this circuit [36] is not quite true, as confirmed by the low $Q_{\mathrm{R}}$ of about 2 at $3 \mathrm{GHz}$ [36].

On the other hand, if $g_{1}$ is sufficiently low, $r_{\mathrm{s}}$ can be low, too. And $Q_{\mathrm{L}}$ is larger than that of the gate- $R$ and its derivatives, because $g_{1}$ generally is much lower than $1 / R_{1}$ in the gate- $R$ circuits. So this circuit could implement an inductor with low series resistance $r_{\mathrm{s}}$. However, there is no easy way of improving $Q_{\mathrm{L}}$ besides reducing $g_{1}$.

The circuit's main drawback is that for the MMIC implementation proposed by the authors, multiple resistors and capacitors are needed to bias the transistors and complete the signal path, increasing parasitic components and making the circuit expensive.

Six months later, the authors reported the results of the same active inductor, with a cascode NMOS inserted to the drain of M2 in Fig. 2-11(a) in an effort to overcome $g_{\mathrm{ds}}$-limitations and increase $Q_{\mathrm{L}}[37,38]$. This over-compensated the circuit loss (mainly because of the extra phase shift from the parasitic poles of the two $G_{\mathrm{M}} \mathrm{S}$ that are now both cascoded, see Section 2.5.3) and yielded a negative resistor element 
in the equivalent circuit. The authors then put a resistor in shunt with the input port to cancel out the negative resistor and obtained a $Q_{\mathrm{R}}$ as high as 65 at $8 \mathrm{GHz}[37,38]$. This method of over-compensating the loss and then introducing another loss element is inefficient in terms of reducing circuit noise and power dissipation.

In 1996, H. Hayashi el al. introduced a similar scheme [26, 27] that overcompensates the circuit loss through the parasitic pole of the cascode transistor $M_{3}$, and then added a series, instead of shunt, resistor to the circuit to compensate the negative equivalent resistor and obtain a high $Q_{\mathrm{L}}$. W. Li et al. proposed a similar method of improving $Q$ [39], using instead an $R-C$ network on the gate of the cascode FET $M_{3}$, and demonstrated a simulated $Q$ near 8000 , which is of little significance in practical applications due to the ultra-high circuit sensitivities, which are proportional to $Q$.

\subsubsection{Cascode CG-CS Active Inductors}

One way of implementing the CG-CS active inductor is shown in Fig. 2-12(a), including the biasing circuit, with $M_{1}$ and $M_{2}$ configured as CG and CS, respectively. Since $I_{2}$ and $I_{3}$ are connected to the same node, $I_{3}$ can be omitted. Then if $I_{\mathrm{DS} 1}=I_{\mathrm{DS} 2}, I_{2}$ can also be omitted. The result is the Cascode CG-CS active inductor. This concept was proposed by Yang et al. using BJTs in 1997 [40] (Fig. 2-12(b)), and then by Wu and Ismail in CMOS technology [41-45] (Fig. 2-12(c)). The equivalent circuit is shown in Fig. 2-12(d). 


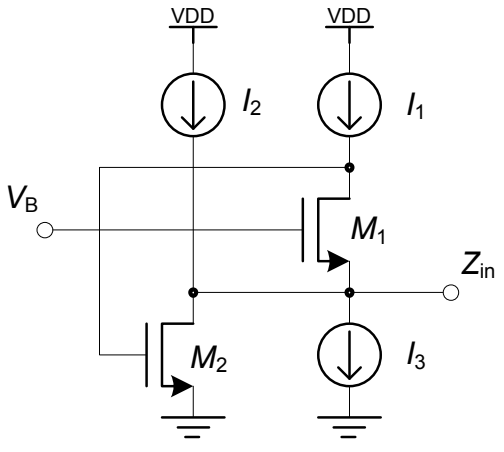

(a)

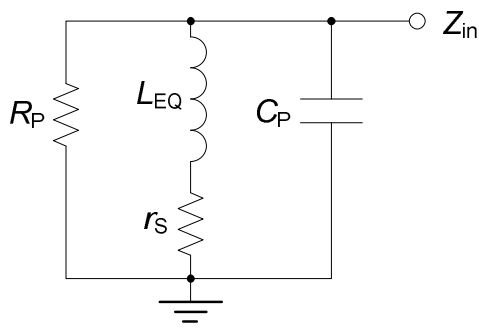

(d)

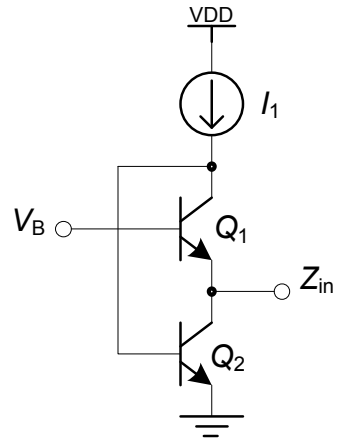

(b)

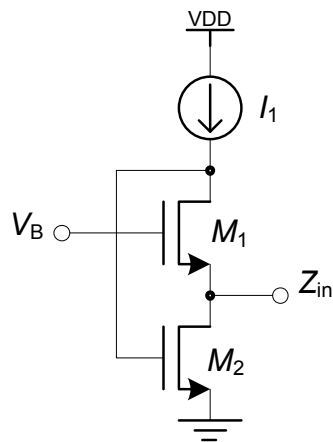

(c)

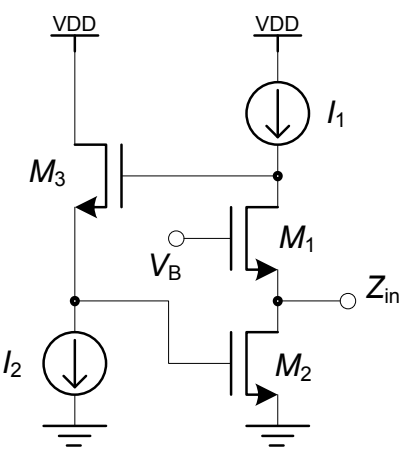

(e)

Figure 2-12 Derivation of the cascode CG-CS active inductor. (a) Circuit derived from signal flow. (b) BJT version. (c) CMOS version. (d) Equivalent circuit. (e) Modified cascode CG-CS.

Aside from a low $Q_{\mathrm{R}}$ that is determined by its CG-CS structure, the circuit's main limitation is the dc biasing. In the CMOS case, we have $V_{\mathrm{GS} 2}=V_{\mathrm{T}, \mathrm{n}}+V_{\mathrm{OD}}=V_{\mathrm{DS} 1}$ $+V_{\mathrm{DS} 2}$. Typically $V_{\mathrm{T}, \mathrm{n}} \approx 0.4-0.6 \mathrm{~V}$, and $V_{\mathrm{DS}}$ has to be greater than $V_{\mathrm{OD}}+100 \mathrm{mV} \approx 0.3$ $\mathrm{V}$, hence the $V_{\mathrm{DS}}$ margins are very limited since $V_{\mathrm{GS} 2}$ is not large, and the circuit may not work across process and temperature corners. A voltage level shifter, which adds parasitic poles and noise, is often used on this circuit to make $V_{\mathrm{DS} 1}+V_{\mathrm{DS} 2}=V_{\mathrm{GS} 2}+$ $\Delta V$. A voltage level shifter implemented by a source follower is shown in Figure 212(e) [46]. Wu and Ismail instead inject extra current to $M_{2}$ through a second current source $I_{2}$ to greatly increase $V_{\mathrm{OD} 2}$ and in turn $V_{\mathrm{GS} 2}$, without increasing $V_{\mathrm{OD} 1}$ and in turn the requirement on $V_{\mathrm{DS} 1}$ (Fig. 2-13). Part of $I_{2}$ is the "recycled" current used by the negative resistor (cross-connected differential pair) for $Q_{\mathrm{R}}$ improvement [45]. $I_{2}$ 
cannot be totally derived from the negative resistor, in order to obtain separate tuning for $Q_{\mathrm{R}}$ and $L_{\mathrm{eq}}$ (frequency).

The active inductor's signal swing is limited by the low $V_{\mathrm{DS}}$ of the FETs. A simple analysis will reveal that similar problem exists in the bipolar case without the use of a level shifter.

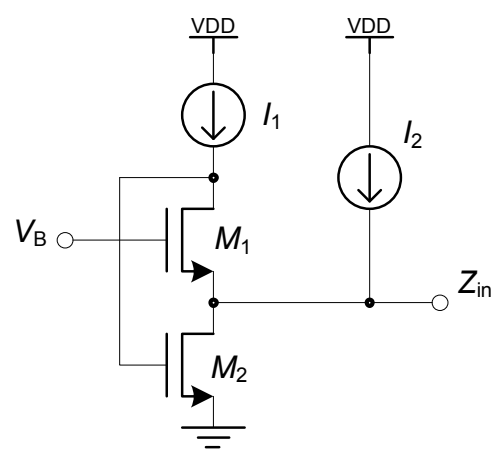

Figure 2-13 Wu and Ismail's solution to dc biasing difficulty: injecting extra current $I_{2}$ into $M_{2}$ to raise $V_{\mathrm{GS} 2}$ without increasing $V_{\mathrm{GS} 1}$, which will increase the $V_{\mathrm{DS} 1}$ requirement and defeat the margin gained through the increase in $V_{\mathrm{GS} 2}$.

\subsubsection{CS-CD Active Inductor and $Q$ Enhancement using $R_{\mathrm{f}}$}

The Common-source common-drain (CS-CD) active inductor [47] is shown in Fig. 214(a). It is fundamental to a popular class of active inductors called "cascode" and "regulated cascode" active inductors. Along the signal path, $M_{1}$ and $M_{2}$ are in CS and CD configuration, respectively, hence its name. It can be roughly explained using the gyrator principle, with $M_{1}$ being the inverting $G_{\mathrm{M}}$ and $M_{2}$ being the noninverting $G_{\mathrm{M}}$, though the load capacitor $C_{\mathrm{gs} 2}$ is connected across the two nodes of gyrator instead of grounded. 


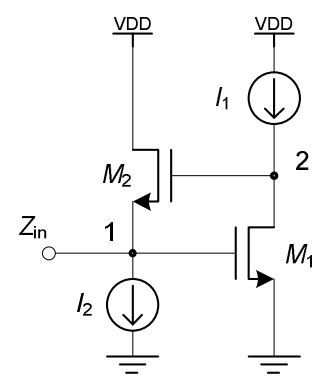

(a)

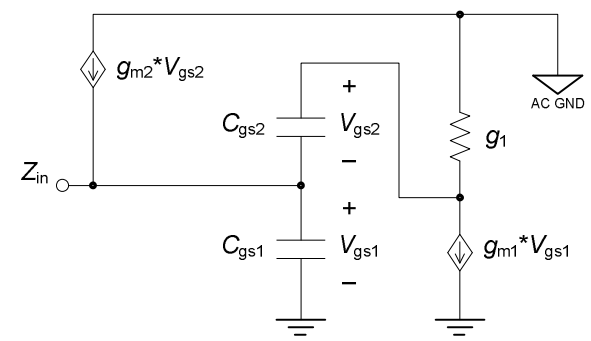

(b)

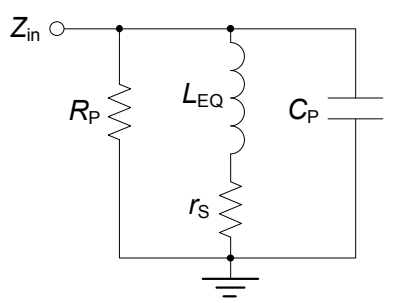

(c)

Figure 2-14 Common-Source Common-Drain (CS-CD) active inductor. (a) The circuit. (b) Small-signal ac equivalent circuit. $g_{1}$ is $g_{\mathrm{ds} 1}+g_{\mathrm{ds}, I_{1}}$. (c) Equivalent circuit showing the inductor.

Small-signal ac analysis reveals that its input impedance is

$$
\begin{aligned}
& Y_{\mathrm{in}}=s C_{\mathrm{gs} 1}+\frac{\left(g_{\mathrm{m} 1}+g_{1}\right)\left(g_{\mathrm{m} 2}+s C_{\mathrm{gs} 2}\right)}{g_{1}+s C_{\mathrm{gs} 2}} \approx s C_{\mathrm{gs} 1}+\frac{g_{\mathrm{m} 1}\left(g_{\mathrm{m} 2}+s C_{\mathrm{gs} 2}\right)}{g_{1}+s C_{\mathrm{gs} 2}} \\
& =s C_{\mathrm{gs} 1}+g_{\mathrm{m} 1}+\frac{g_{\mathrm{m} 1} g_{\mathrm{m} 2}-g_{\mathrm{m} 1} g_{1}}{g_{1}+s C_{\mathrm{gs} 2}} \approx s C_{\mathrm{gs} 1}+g_{\mathrm{m} 1}+\frac{g_{\mathrm{m} 1} g_{\mathrm{m} 2}}{g_{1}+s C_{\mathrm{gs} 2}} \\
& =s C_{\mathrm{gs} 1}+g_{\mathrm{m} 1}+\frac{1}{\frac{g_{1}}{g_{\mathrm{m} 1} g_{\mathrm{m} 2}}+s \frac{C_{\mathrm{gs} 2}}{g_{\mathrm{m} 1} g_{\mathrm{m} 2}}}
\end{aligned}
$$

which can be represented by the equivalent circuit shown in Fig. 2-14(c). The equivalent elements are

$$
R_{\mathrm{p}}=\frac{1}{g_{\mathrm{m} 1}}, C_{\mathrm{p}}=C_{\mathrm{gs} 1}, L_{\mathrm{eq}}=\frac{C_{\mathrm{gs} 2}}{g_{\mathrm{m} 1} g_{\mathrm{m} 2}}, r_{\mathrm{s}}=\frac{g_{1}}{g_{\mathrm{m} 1} g_{\mathrm{m} 2}}
$$

If $g_{1}$ is low, $r_{\mathrm{s}}$ can be very low; however, $R_{\mathrm{p}}=1 / g_{\mathrm{m} 1}$ is a low shunt resistance, and consequently, the CS-CD active inductor has an average $Q_{\mathrm{L}}$ but very low $Q_{\mathrm{R}}$. Nonetheless, this circuit is readily biased, with a low $V_{\mathrm{DD}}$ requirement and a decent maximum signal swing.

It can be shown that the CS-CD circuit exhibits a low- $Q$ lowpass filter characteristic from Node 1 to Node 2 in Fig. 2-14(a). The input impedance is low (= $\left.1 / g_{\mathrm{m} 1}\right)$ and can be $50 \Omega$ when properly designed. These two characteristics make CS- 
$\mathrm{CD}$ as well as its derivatives, cascode and regulated cascode active inductors, suitable for the low-noise amplifiers (LNAs) in broadband wireless communications [47-50], even though the "low-noise" designation is not entirely true, if compared with LNAs designed with passive inductors. The transfer function is a broadband lowpass; in contrast LNAs using passive inductors are always narrow-band bandpass. The broadband property also allows more noise at different frequencies pass through, so it should be used judiciously.

A common method of enhancing the low $Q_{\mathrm{R}}$ is to insert a resistor $R_{\mathrm{f}}$ in the signal loop [51-53], as shown in Fig. 2-15(b). We can include $R_{\mathrm{f}}$ in the equivalent circuit in Fig. 2-14(b) and reveal its effect through circuit analysis; however, a quicker way to understand the function is to recognize that $R_{\mathrm{f}}$, together with $C_{\mathrm{gs} 2}$, creates a phase lag in $V_{\mathrm{gs} 2}$ and in turn in $I_{\mathrm{d} 2}$. As we shall see later in Section 2.5.3, a phase lag in the gyrator loop has the effect of increasing $Q_{\mathrm{R}}$. However, there are two issues associated with this:

(1) Not all processes have large-value resistors available.

(2) Since $C_{\mathrm{gs} 2}$ is very small, to create an appreciable phase lag $\left(\sim 5-15^{\circ}\right)$, a resistor on the order of a few hundred $\mathrm{k} \Omega$ may be needed, and this means a large layout area. In reality, however, the required value of $R_{\mathrm{f}}$ is usually a little lower, because the poly resistors have distributed $R$ and $C$, and create extra phase lag. 


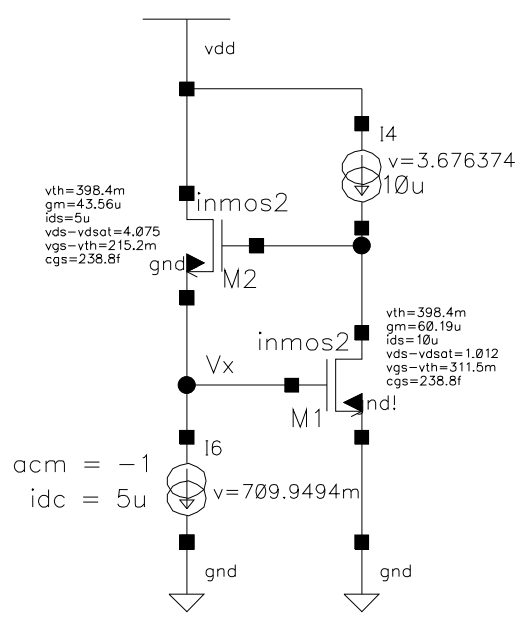

(a)

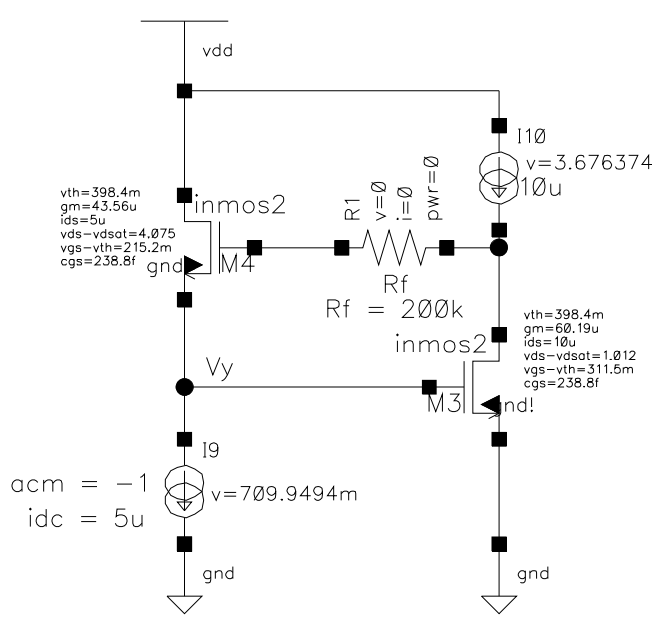

(b)

Figure 2-15 (a) A CS-CD active inductor simulation circuit. $g_{\mathrm{ds} 1}=0.597 \mathrm{pS}$. (b) With a $Q$-enhancing resistor $R_{\mathrm{f}}$.

The circuits in Fig. 2-15 were simulated and their $R_{\mathrm{p}}$ are shown in Fig. 2-16. For the circuit in Fig. 2-15(a), using Eq. (2.24) and the simulated operating points shown, we can calculate $R_{\mathrm{p}}=16.6 \mathrm{k} \Omega, L_{\mathrm{eq}}=91 \mu \mathrm{H}$, and $r_{\mathrm{s}}=0.23 \mathrm{~m} \Omega$; these numbers are close to the simulated results, which are $R_{\mathrm{p}}=16.7 \mathrm{k} \Omega, L_{\mathrm{eq}}=97.7 \mu \mathrm{H}$, and $r_{\mathrm{s}}=0.63$ $\mathrm{m} \Omega$.

Comparing the simulation results we find that $R_{\mathrm{f}}$ does improve $Q$, as measured by the $R_{\mathrm{p}}$ increase from $16.8 \mathrm{k} \Omega$ (cursor A) to $22.4 \mathrm{k} \Omega$ (cursor $\mathrm{B}$ ). Since $Q_{\mathrm{R}}$ is proportional to $R_{\mathrm{P}}$ to the first order (Eq. 2.8), $Q_{\mathrm{R}}$ will increase by about $35 \%$. $R_{\mathrm{f}}$ does not change the value of $L_{\mathrm{eq}}$ to the first order.

This method of creating a phase lag by inserting $R_{\mathrm{f}}$ to enhance $Q_{\mathrm{R}}$ is also used in cascode and regulated cascode, as well as differential active inductors, as we shall see later. 


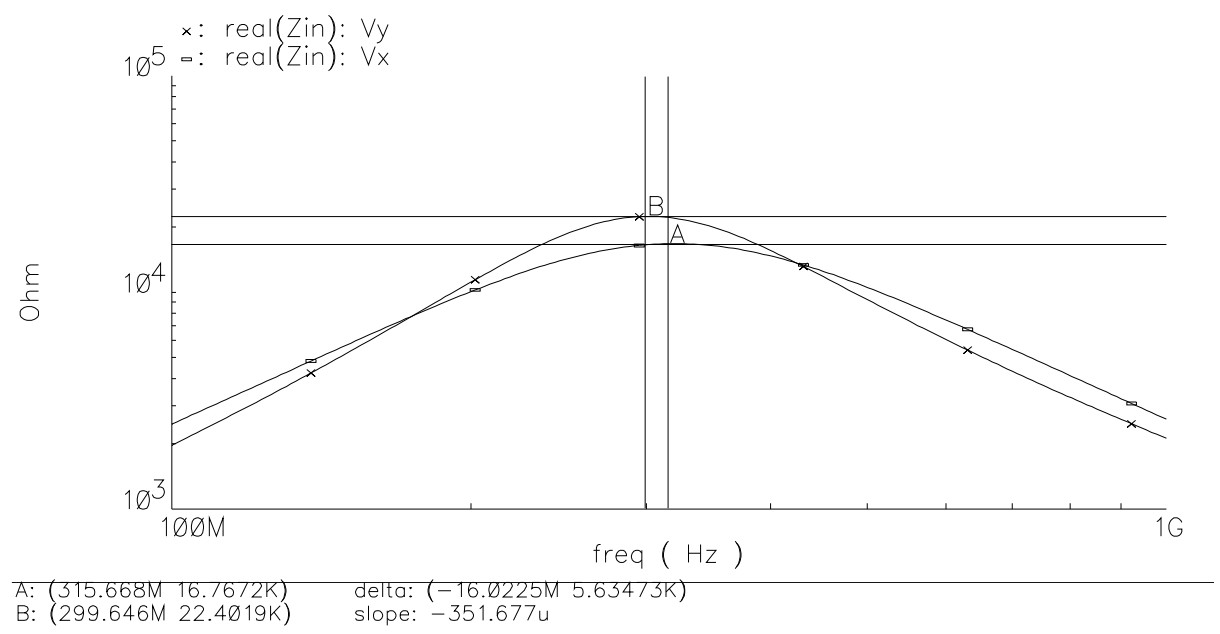

Figure 2-16 Simulated $R_{\mathrm{p}}$ of the circuits in Fig. 2-15. $V_{\mathrm{x}}$ and $V_{\mathrm{y}}$ are from the circuits in Fig. 2-15(a) and (b), respectively.

\subsubsection{Cascode and Regulated-Cascode Active Inductors}

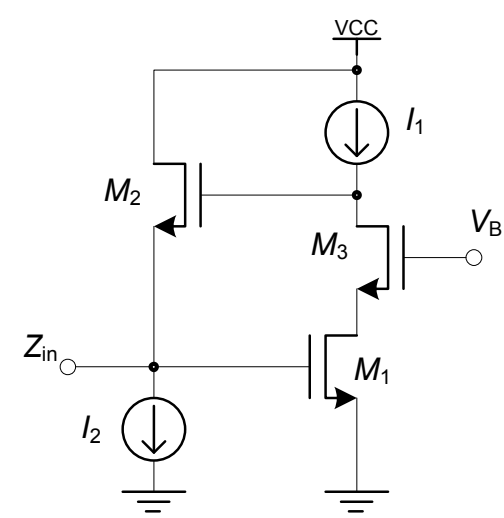

(a)

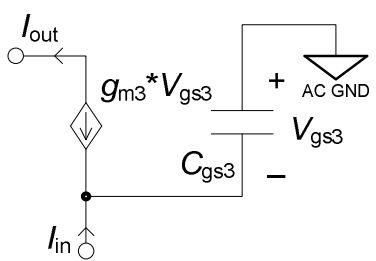

(b)

Figure 2-17 (a) Cascode active inductor. (b) small-signal equivalent circuit of $M_{3}$.

As seen from Eq. (2.24), to reduce the series resistor $r_{\mathrm{s}}$ of the CS-CD circuit we need to reduce $g_{1}$. In an effort to reduce $g_{1}$, in many designs [51, 53-59] a cascode transistor is introduced to the drain of $M_{1}$ of the CS-CD active inductor, resulting in the cascode active inductor as shown in Fig. 2-17(a). The common understanding is that the cascode NFET increases $R_{\text {out }}$ looking into the $M_{1}$ drain and hence increases $Q_{\mathrm{L}}$ and $Q_{\mathrm{R}}$. However, there are two apparent paradoxes to this claim: 
1. The PFET implementing the biasing current $I_{1}$ is usually not cascoded, limiting $g_{1}$ by the PFET's $g_{\mathrm{ds}}$. The introduction of the cascode NFET $M_{3}$ will therefore reduce $g_{1}$ by a maximum of about $50 \%$.

2. Per Eq. (2.24), $g_{1}$ is only related to the series resistance $r_{\mathrm{s}}$. Reducing $g_{1}$ does not affect the low-value parallel resistor $R_{\mathrm{p}}$ [57], which limits the $Q_{\mathrm{R}}$ to a low value. Nonetheless, the cascode scheme does improve $Q_{\mathrm{R}}$ and compensates for the parallel resistance $R_{\mathrm{p}}$, as verified in many papers. The true reason is that the cascode transistor $M_{3}$ creates a phase lag between its input and output current. Using the smallsignal ac circuit in Fig. 2-17(b), the relationship is

$$
\frac{I_{\text {out }}}{I_{\text {in }}}=\frac{1}{1+s C_{\mathrm{gs} 3} / g_{\mathrm{m} 3}}
$$

The active inductor $f_{\mathrm{R}}$ is usually $5-40 \%$ that of $g_{\mathrm{m}} / C_{\mathrm{gs}}$ of the FETs used, hence the cascode FET current transfer function pole frequency is conveniently located at a frequency of 2-20 times that of $f_{\mathrm{R}}$, with a phase lag of $3-20^{\circ}$ at $f_{\mathrm{R}}$. As we will see later in Section 2.5.3, this is the right amount of phase lag for $Q_{\mathrm{R}}$ compensation. Therefore, cascode is an effective way of improving the CS-CD active-inductor $Q_{\mathrm{R}}$, not because that it increases $R_{\text {out }}$ of the $G_{\mathrm{m}}$ cell, but because of its phase lag from the highfrequency parasitic pole. Note that the cascode FET does not significantly reduce $r_{\mathrm{s}}$ and improve $Q_{\mathrm{L}}$, as it cannot reduce $g_{1}$ much without inserting a cascode PFET to the current source $I_{1}$, which will increase the minimum $V_{\mathrm{DD}}$ requirement.

J. Yang et al. insert a gate-biasing resistor $R_{\mathrm{B}}$ (of unknown value) to the cascode circuit (Fig. 2-18(a)) and augment $C_{\mathrm{gs} 3}$ by putting a capacitor between $M_{3}$ 's gate and source, and claim that this compensates the device losses [58]. Using the ac small- 
signal equivalent circuit of $M_{3}$ in Fig. 2-18(b), we have

$$
\begin{aligned}
& \left(I_{\text {in }}-I_{\text {out }}\right) \frac{1}{s C_{\mathrm{gs} 3}} g_{\mathrm{m} 3}=I_{\text {out }}, \text { and } \\
& \frac{I_{\text {out }}}{I_{\text {in }}}=\frac{g_{\mathrm{m} 3}}{s C_{\mathrm{gs} 3}+g_{\mathrm{m} 3}}
\end{aligned}
$$

Comparing Eqs. (2.25) and (2.26), we find that $R_{\mathrm{b}}$ has no direct effect on the current transfer function of $M_{3}$, and therefore the circuit performance is not significantly different from that in Fig. 2-17. $R_{\mathrm{b}}$ only works through the $2^{\text {nd }}$-order effect from the $g_{\mathrm{ds}}$ of $M_{3}$. Nonetheless, $R_{\mathrm{b}}$ exists on most real circuits because the output resistance of biasing circuits is limited.

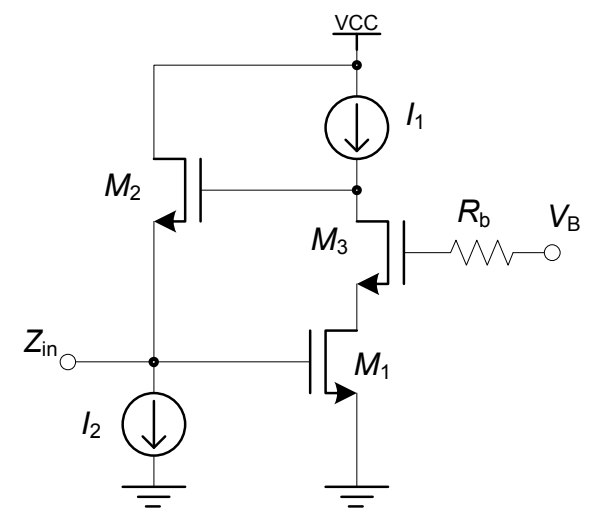

(a)

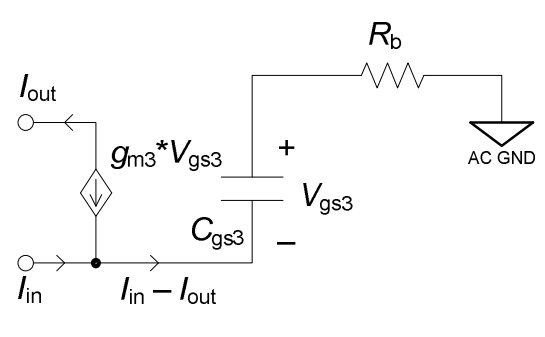

(b)

Figure 2-18 (a) Adding $R_{\mathrm{b}}$ to the cascode active inductor. (b) Small-signal equivalent circuit of $M_{3}$ and $R_{\mathrm{b}}$.

To examine the $Q$ enhancement of the cascode FET, a comparison simulation is performed on the circuits in Fig. 2-19. Note that ideal current sources are used, and the $r_{\mathrm{s}}$ reduction from the use of cascode is exaggerated because there is no current source leakage to dilute the $Q$ enhancement. The simulation results in Fig. 2-20 show that $R_{\mathrm{p}}$ is increased by $\sim 5.5 \mathrm{x}\left(R_{\mathrm{P}}\right.$ ratio $\left.=92.5 \mathrm{k} \Omega / 16.8 \mathrm{k} \Omega\right)$. 

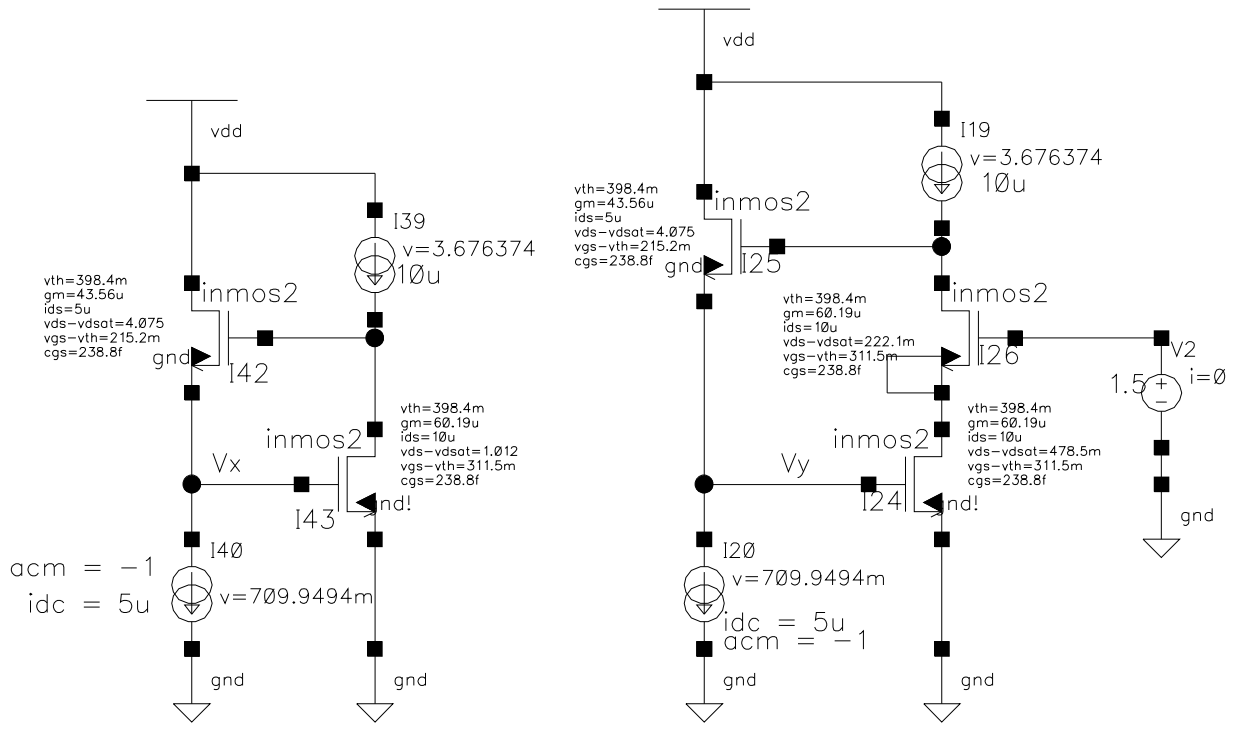

Figure 2-19 Simulation circuits to show the $Q$ enhancement achieved by cascode. (a) CS-CD active inductor; (b) Cascode active inductor.

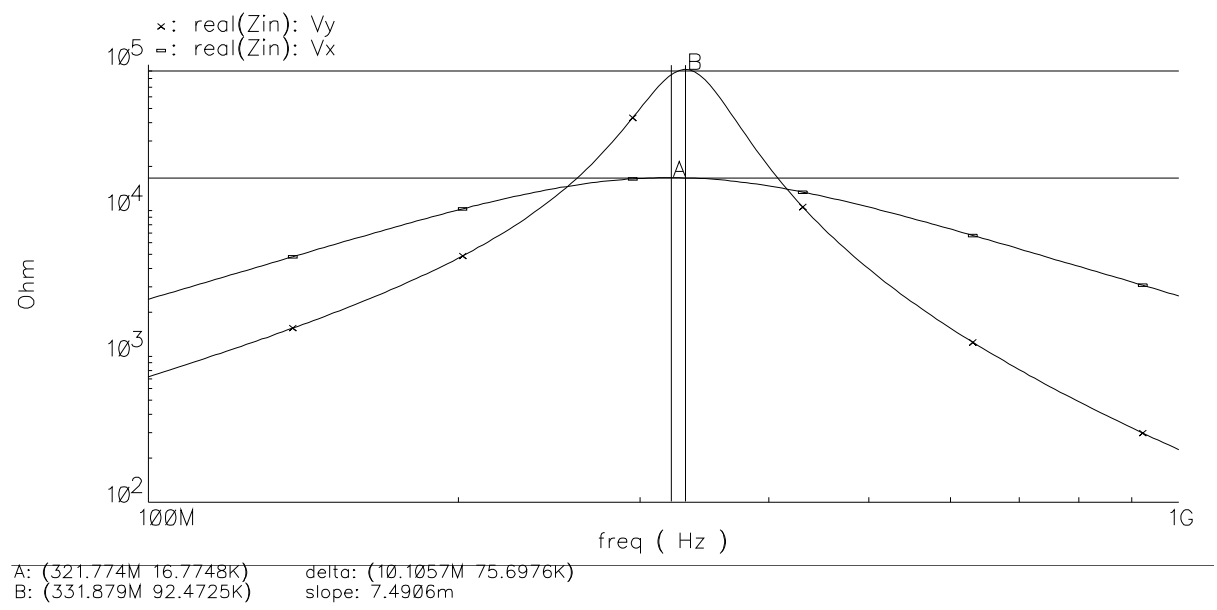

Figure 2-20 Simulated $R_{\mathrm{p}}$ of the circuits in Fig. 2-19. Cursor A and B are for circuit (a) and (b), respectively.

Finally, by introducing $M_{4}$ and $I_{3}$ to regulate the $M_{3}$ gate voltage, the regulated cascode active inductor can be derived $[53,55,60]$, as shown in Fig. 2-21. $M_{4}$ is biased at a fixed $I_{\mathrm{DS} 4}=I_{3}$, thus $V_{\mathrm{GS} 4}$, and in turn, $V_{\mathrm{DS} 1}$, is constant, increasing the resistance looking into the $M_{1}$ drain. The cascode FET $M_{3}$ further amplifies the resistance looking into the drain of $M_{3}$. The voltage regulation through $M_{4}$ only makes $M_{3}$ more effective. 
The regulated cascode active inductor can be understood as a CS-CD active inductor $+g_{1}$ reduction through cascode + further $g_{1}$ reduction from voltage regulation. Note that $g_{1}$ is limited by the biasing current $I_{1}$ in Fig. 2-17(a) when the resistance looking down into the cascode FET is very high, therefore, the improvement from voltage regulation will be negligible if the cascode active inductor is designed properly in the first place. The efficacy in improving $Q_{\mathrm{R}}$ researchers are seeing from the use of voltage regulation is more likely due to the extra currenttransfer-function delay in the cascode FET from the voltage regulation, rather than due to the $g_{1}$ reduction from the use of voltage regulation.

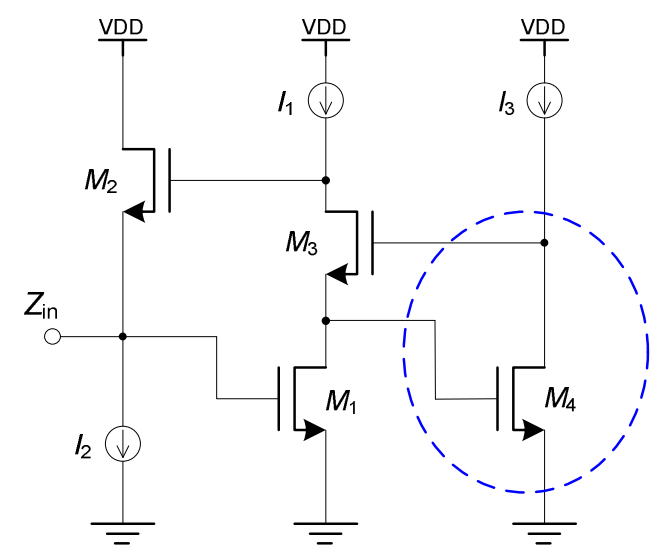

Figure 2-21 Regulated cascode active inductor circuit.

\subsubsection{Differential-Pair Active Inductor}

The differential-pair active inductor [61-64] is shown in Fig. 2-22(a). The differential pair is the simplest differential $G_{\mathrm{M}}$, and using the $G_{\mathrm{M}}$ symbol, the circuit is redrawn in Fig. 2-22(b). The circuit is recognized as a gyrator, with $C_{1}$ and $C_{2}$ being the parasitic capacitance (mainly $C_{\mathrm{gs}}$ ) across the drains of $M_{1,2}$ and $M_{3,4}$, respectively. The noninverting $G_{\mathrm{M}}$ is conveniently obtained by a cross connecting of one of the $G_{\mathrm{M}}$ 
cells.

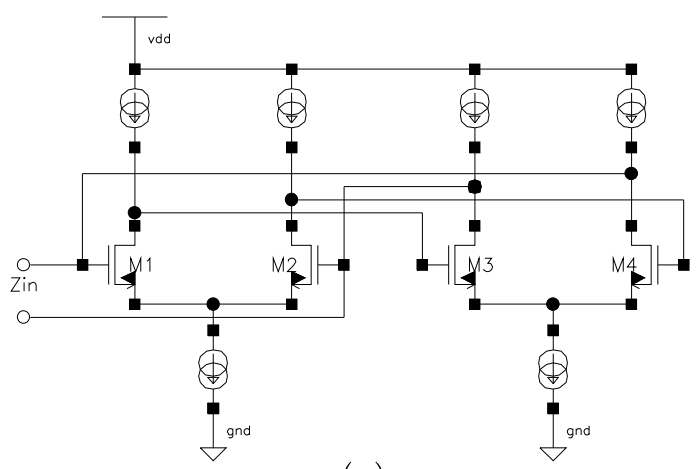

(a)
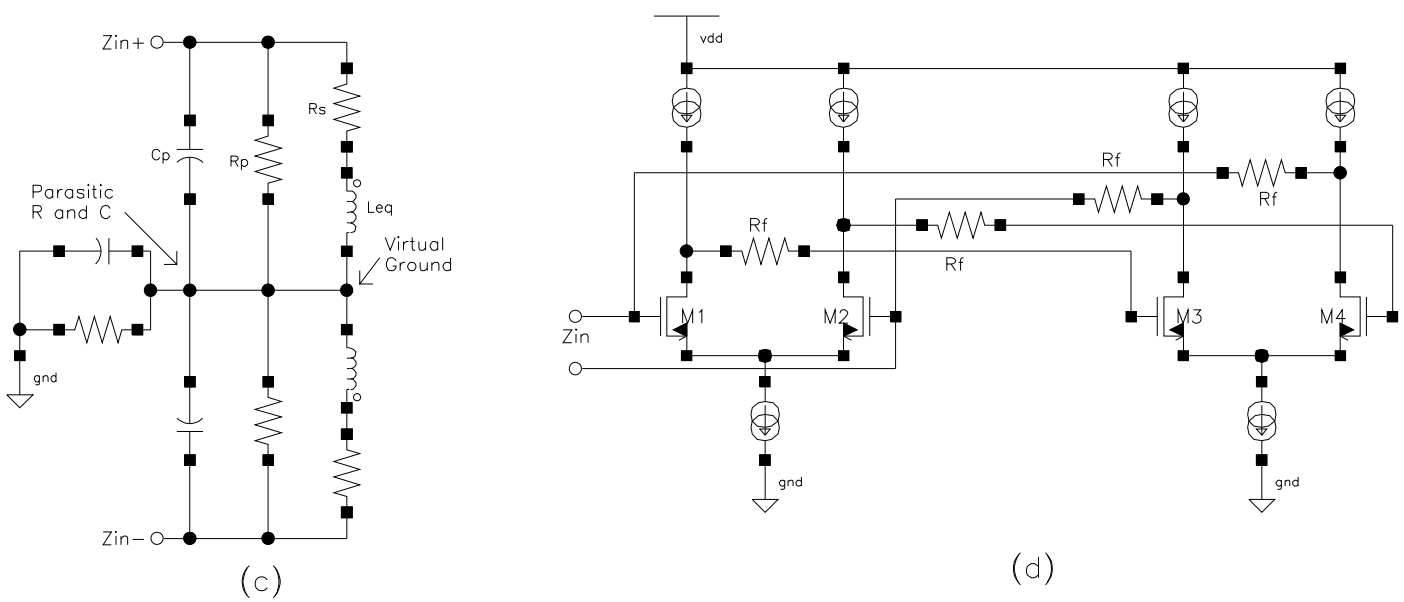

(d)

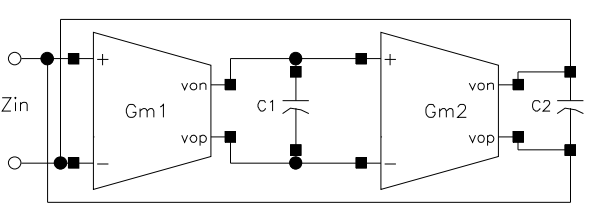

(b)

Figure 2-22 The differential-pair active inductor.

The circuit shall not be erroneously interpreted as a floating inductor, even though it may roughly operate as one. There is a virtual ground node between the two input terminals, as shown in Fig. 2-22(c), if the circuit is treated as a floating inductor, the virtual ground node will become a signal node, with a parasitic capacitor, so it will not be a good floating inductor.

The differential-pair active inductor does not have a low-impedance node in its gyrator loop, hence it can achieve a reasonably high $Q_{\mathrm{R}}$ without compensation. To further compensate for the device losses, a negative resistor made with a crossconnected differential pair can be introduced to the circuit [64]. However, very often 
the circuit is overly compensated and becomes unstable, because its $g_{1}$ and $g_{\mathrm{L}}$ are both from $g_{\mathrm{ds}} \mathrm{s}$, which are orders of magnitude smaller than $g_{\mathrm{m}}$. A more common method is to add some feedback resistors in series with the gates [61-63], as shown in Fig. 222(d). $R_{\mathrm{f}}$, together with the $C_{\mathrm{gs}}$ it connects to, creates a phase lag in the gyrator loop. As we shall see later in Section 2.5.3, a phase lag in the gyrator loop has the effect of increasing $Q_{\mathrm{R}}$.

The major drawback of this circuit is the difficulty of dc biasing, because it has positive feedback for common-mode voltages with loop gain much greater than 1 . It can be found from Fig. 2-22(a) that the common-mode signal path consists of two inverters in a loop, which has a large positive gain. Special measures such as biasing resistors must be used to stabilize the dc biasing, which as a result, degrade the circuit's performance.

\subsubsection{Karsilayan's Active Inductor}

During the design of a floating tunable voltage source for high-linearity $G_{\mathrm{M}}$ cells, Karsilayan and Schaumann [65] discovered that the circuit shown in Fig. 2-23(a) is in fact a high- $Q$ active inductor. The circuit is easily understood using the gyrator principle. The differential pair formed by $M_{1}$ and $M_{2}$ is a noninverting $G_{\mathrm{M}}$ cell with input voltage at node 1 and output current at node 3 , and $M_{3}$ is an inverting $G_{\mathrm{M}}$ with input voltage at node 3 and output current at node 1 . The parasitic capacitance, mainly $C_{\mathrm{gs} 3}$, is thus gyrated into an equivalent inductor at node 1 . Due to the inevitable

parasitic capacitance at node 1 and the circuit losses, the equivalent circuit is a resonator, as shown in Fig. 2-23(b). The series resistor originates from the load loss $g_{\mathrm{L}}$ 
at node 3 .

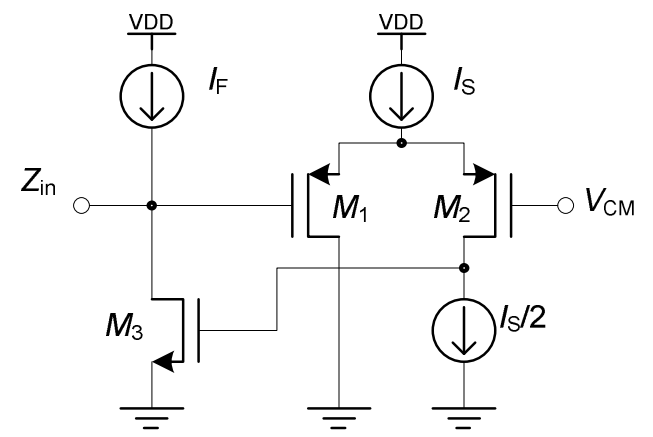

(a)

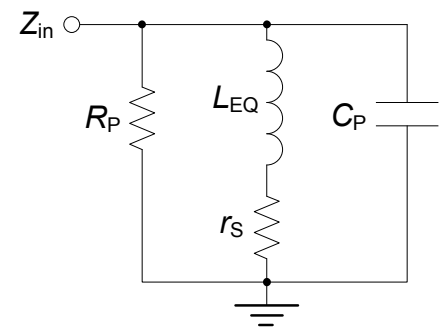

(b)

Figure 2-23 (a) Karsilayan's active inductor circuit. $V_{\mathrm{CM}}$ is a de voltage that sets the circuit's commonmode voltage. (b) Small-signal ac equivalent circuit.

Karsilayan's circuit, like the regulated cascode circuit, is readily biased, with low $V_{\mathrm{DD}}$ requirement $\left(2 V_{\mathrm{DS}}+V_{\mathrm{GS}}\right)$. Unlike the regulated cascode circuit and many other active inductors, the two nodes in the gyrator loop are both high-resistance, and consequently, the circuit has an intrinsically high $Q_{\mathrm{R}}$. It was also proved that the unavoidable device losses are readily compensated by tuning the parasitic capacitance at node $2, C_{2}$, via a varactor [65]. When $C_{2}$ increases, the phase lag of $M_{1}-M_{2}$ differential-pair $G_{\mathrm{M}}$ cell increases, and this has the effect of increasing the $Q_{\mathrm{R}}$.

It can be shown that if node 1 is driven by a current source, $I_{\text {in }}$, the output voltage at node 3 has lowpass characteristic. If the output is taken instead at node 1 , a bandpass function, $V_{\text {out }}=I_{\text {in }} Z_{\text {in }}$, is obtained. Alternatively, if the input voltage signal is injected at node $V_{\mathrm{CM}}$ together with a dc-voltage $V_{\mathrm{CM}}$, a lowpass voltage output can be derived at node 1 [66]. The active inductor circuit has also been used to design sinusoidal RF oscillators [67]. 


\subsubsection{Other Active Inductors}

There are many other active inductor circuits [68-79] that are variations of the basic topologies shown above, for example, by exchanging NFET with PFET in part or all of the circuits, combining the $Q$-enhancement techniques, etc. However, they can almost always be simplified down to the gyrator equivalent circuit shown in Fig. 2-3. The actual circuit can have more equivalent devices than that; however, the benefits from the extra devices rarely leverage the entailed disadvantages, such as lower $f_{\mathrm{R}}$ from extra parasitic capacitance, more parasitic poles/zeros from extra circuit nodes, higher noise, and more power dissipation.

\subsection{Methods of Increasing $Q_{\mathrm{R}}$}

There are three approaches for increasing $Q_{\mathrm{R}}$ : reducing $g_{1}$ and/or $g_{\mathrm{L}}$ in the gyrator equivalent circuit; introducing a negative resistor; and generating phase lag. It shall be noted that per Eq. (2.6), $r_{\mathrm{S}}$ is determined by $g_{\mathrm{L}}$, which is the loss at the load node, and the only way to reduce $r_{\mathrm{S}}$ and increase $Q_{\mathrm{L}}$ is to reduce $g_{\mathrm{L}}$. Phase lag in the gyrator loop will not reduce $r_{\mathrm{S}}$ and improve $Q_{\mathrm{L}}$.

\subsubsection{Reducing $g_{1}$ and/or $g_{\mathrm{L}}$ in Gyrator Equivalent Circuit}

From Eqs. (2.6) and (2.10) we have

$$
Q_{\mathrm{L}}(\omega) \approx \frac{\omega L_{\mathrm{eq}}}{r_{\mathrm{s}}}=\frac{\omega C_{\mathrm{L}} /\left(G_{\mathrm{M} 1} G_{\mathrm{M} 2}\right)}{g_{\mathrm{L}} /\left(G_{\mathrm{M} 1} G_{\mathrm{M} 2}\right)}=\frac{\omega C_{\mathrm{L}}}{g_{\mathrm{L}}}
$$

that is, to increase $Q_{\mathrm{L}}$ at a certain frequency we must increase $C_{\mathrm{L}} / g_{\mathrm{L}}$. Usually, the value of $C_{\mathrm{L}}$ is kept the same, but through circuit techniques $g_{\mathrm{L}}$ is reduced. Note that all 
circuits can be scaled up and down, but it is the ratio that matters. This is a very common method for enhancing $Q$ in many circuits, such as in the cascode active inductor. Note that reducing $g_{\mathrm{L}}$ alone is sufficient to enhance $Q_{\mathrm{L}}$, because as an inductor the circuit operates at a lower frequency than as a resonator, and the effect of $g_{\mathrm{L}}$ dominates that of $g_{1}$ in determining $Q_{\mathrm{L}}$.

On the other hand, reducing $g_{\mathrm{L}}$ is necessary, but not sufficient to obtain a high $Q_{\mathrm{R}}$, that is, a high- $Q$ resonator, to implement designs such as narrow-band bandpass filters. From Eqs. (2.6) and (2.8) we have

$$
Q_{\mathrm{R}}=\frac{\sqrt{\frac{1+g_{1} r_{\mathrm{S}}}{L_{\mathrm{eq}}}}}{\frac{r_{\mathrm{s}}}{L_{\mathrm{eq}}}+\frac{g_{1}}{C_{1}}} \approx \frac{\sqrt{L_{\mathrm{eq}} C_{1}}}{r_{\mathrm{s}} C_{1}+g_{1} L_{\mathrm{eq}}}=\frac{\sqrt{\frac{G_{\mathrm{M}} G_{\mathrm{M} 2}}{C_{1} C_{\mathrm{L}}}}}{\frac{g_{\mathrm{L}}}{C_{\mathrm{L}}}+\frac{g_{1}}{C_{1}}}=\frac{\omega_{0}}{\frac{g_{\mathrm{L}}}{C_{\mathrm{L}}}+\frac{g_{1}}{C_{1}}}
$$

$Q_{\mathrm{R}}$ is jointly determined by $g_{\mathrm{L}}$ and $g_{1}$. To enhance $Q_{\mathrm{R}}$, both $g_{\mathrm{L}}$ and $g_{1}$ must be reduced, a simple requirement, but not always easy to realize.

\subsubsection{Negative Resistors}

Many designers use negative resistors, such as cross-connected differential pairs, to cancel out the effect of $g_{1}$ or $g_{\mathrm{L}}$, or both, to improve $Q$. There are too many references to be included here. One example is [45]. Negative resistors consume power and introduce nonlinearities and noise; however, this method is easy to implement, and for some circuit structures, is the only option.

\subsubsection{Phase Lag in the Gyrator Loop}

Another way to enhance $Q_{\mathrm{R}}$ is to introduce a phase lag into the gyrator loop. This is a widely used method, but not always so realized by designers. For example, many 
papers attribute the higher $Q_{\mathrm{R}}$ of the cascode-active inductor solely to the $R_{\text {out }}$ enhancement of the cascode FET [57], and some researchers believe that the phase lag decreases $Q$ and hence is undesirable [59]. The following discussion will show that the opposite is true.

Figure 2-3 assumes that both $G_{\mathrm{M} 1}$ and $G_{\mathrm{M} 2}$ have zero phase shift at all frequencies concerned. This is not true in practice. The inverting $G_{\mathrm{M}}$ can be implemented by a single common-source FET and has almost zero phase shift; however, due to transistor (either FET or BJT) properties, a noninverting $G_{\mathrm{M}}$ has to be a common-gate (cascode) FET, which yields low- $Q$ circuits, or a differential pair [65] (= folded cascode), and has inevitably a phase lag due to the parasitic pole(s). Note that a single CS FET may have a phase advance at high frequencies due to $C_{\mathrm{gd}}$.

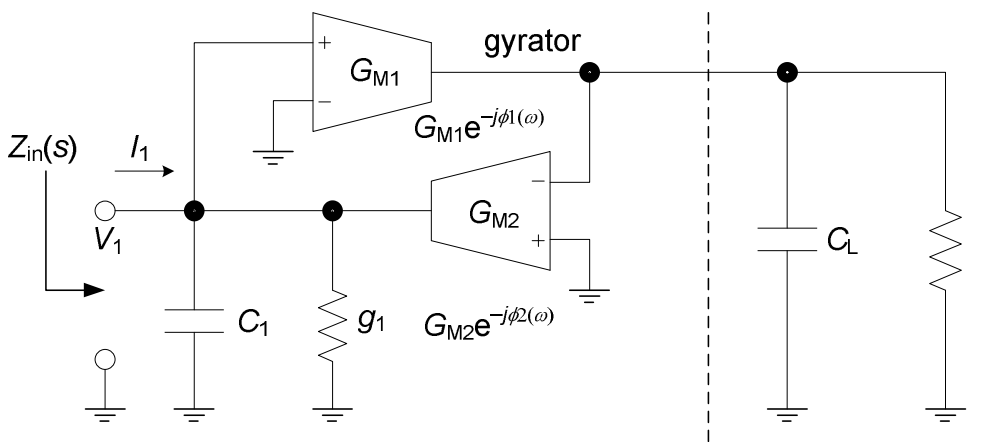

Figure 2-24 The gyrator equivalent circuit of an active inductor. The phase lags of the $G_{\mathrm{M}}$ cells are shown to analyze their effect on $Q$.

The gyrator equivalent circuit including the $G_{\mathrm{M}}$ phase shifts is shown in Fig. 224 , where each $G_{\mathrm{M}}$ cell has a frequency-dependent phase lag. The expression for input admittance is

$$
Y_{\text {in }}=s C_{1}+g_{1}+\frac{G_{\mathrm{M} 1} e^{-j \phi_{1}(\omega)} G_{\mathrm{M} 2} e^{-j \phi_{2}(\omega)}}{g_{\mathrm{L}}+s C_{\mathrm{L}}}=s C_{1}+g_{1}+\frac{G_{\mathrm{M} 1} G_{\mathrm{M} 2} e^{-j \phi(\omega)}}{g_{\mathrm{L}}+s C_{\mathrm{L}}}
$$


where

$$
\phi(\omega)=\phi_{1}(\omega)+\phi_{2}(\omega)
$$

We first concentrate on the last term of $Y_{\text {in }}$ and name it $Y^{\prime}{ }_{\text {in }}=1 / Z^{\prime}{ }_{\text {in }}$,

$$
\begin{aligned}
& Z_{\text {in }}^{\prime}=\frac{g_{\mathrm{L}}+s C_{\mathrm{L}}}{G_{\mathrm{M} 1} G_{\mathrm{M} 2} e^{-j \phi(\omega)}}=\frac{g_{\mathrm{L}}+s C_{\mathrm{L}}}{G_{\mathrm{M} 1} G_{\mathrm{M} 2}[\cos \phi(\omega)-j \sin \phi(\omega)]} \\
& =\frac{\left(g_{\mathrm{L}}+j \omega C_{\mathrm{L}}\right)[\cos \phi(\omega)+j \sin \phi(\omega)]}{G_{\mathrm{M} 1} G_{\mathrm{M} 2}} \\
& =\frac{j \omega\left(C_{\mathrm{L}} \cos \phi+\frac{g_{\mathrm{L}} \sin \phi}{\omega}\right)+g_{\mathrm{L}} \cos \phi-\omega C_{\mathrm{L}} \sin \phi}{G_{\mathrm{M} 1} G_{\mathrm{M} 2}} \\
& =j \omega L_{\mathrm{eq}}+r_{\mathrm{s}}
\end{aligned}
$$

where

$$
L_{\mathrm{eq}}=\frac{C_{\mathrm{L}} \cos \phi(\omega)+\frac{g_{\mathrm{L}} \sin \phi(\omega)}{\omega}}{G_{\mathrm{M} 1} G_{\mathrm{M} 2}}, \quad r_{\mathrm{s}}=\frac{g_{\mathrm{L}} \cos \phi(\omega)-\omega C_{\mathrm{L}} \sin \phi(\omega)}{G_{\mathrm{M} 1} G_{\mathrm{M} 2}}
$$

Therefore the circuit is equivalent to that in Fig. 2-23(b) with $C_{\mathrm{P}}=C_{1}, R_{\mathrm{P}}=1 / g_{1}$, and $L_{\text {eq }}$ and $r_{\mathrm{S}}$ given in Eq. (2.32).

The inductor $Q$ is

$$
Q_{\mathrm{L}}(\omega)=\frac{j \omega L_{\mathrm{eq}}}{r_{\mathrm{s}}}=j \omega \frac{C_{\mathrm{L}} \cos \phi(\omega)+\frac{g_{\mathrm{L}} \sin \phi(\omega)}{\omega}}{g_{\mathrm{L}} \cos \phi(\omega)-\omega C_{\mathrm{L}} \sin \phi(\omega)}
$$

When $\phi=0, Q_{\mathrm{L}}$ is identical to that in Eq. (2.27). As $\phi$ gradually increases, $Q_{\mathrm{L}}$ will increase, too, because of the cancellation in the denominator. When

$$
\phi(\omega)=\tan ^{-1} \frac{g_{\mathrm{L}}}{\omega C_{\mathrm{L}}}
$$

$Q_{\mathrm{L}}=\infty$. This conclusion is contrary to that in [59], which states that the phase lag will reduce $Q$. On the other hand, $\phi(\omega)$ is generally derived from poles, and rarely matches 
the $\tan ^{-1}(1 / \omega)$ curve. So this compensation scheme for active inductors when used as inductors can only work over a narrow frequency range.

The input impedance can also be written as

$$
\begin{aligned}
& Z_{\text {in }}=\frac{1}{g_{1}+s C_{1}+\frac{G_{\mathrm{M} 1} G_{\mathrm{M} 2} e^{-j \phi(\omega)}}{g_{\mathrm{L}}+s C_{\mathrm{L}}}} \\
& =\frac{g_{\mathrm{L}}+s C_{\mathrm{L}}}{s^{2} C_{1} C_{\mathrm{L}}+s\left(C_{\mathrm{L}} g_{1}+C_{1} g_{\mathrm{L}}-\frac{G_{\mathrm{M} 1} G_{\mathrm{M} 2} \sin \phi}{\omega}\right)+g_{1} g_{\mathrm{L}}+G_{\mathrm{M} 1} G_{\mathrm{M} 2} \cos \phi} \\
& =\frac{\frac{s}{C_{1}}+\frac{g_{\mathrm{L}}}{C_{1} C_{\mathrm{L}}}}{s^{2}+s\left(\frac{g_{1}}{C_{1}}+\frac{g_{\mathrm{L}}}{C_{\mathrm{L}}}-\frac{G_{\mathrm{M} 1} G_{\mathrm{M} 2} \sin \phi}{\omega C_{1} C_{\mathrm{L}}}\right)+\frac{g_{1} g_{\mathrm{L}}+G_{\mathrm{M} 1} G_{\mathrm{M} 2} \cos \phi}{C_{1} C_{\mathrm{L}}}}=\frac{\frac{s}{C_{1}}+\frac{g_{\mathrm{L}}}{C_{1} C_{\mathrm{L}}}}{s^{2}+s \frac{\omega_{\mathrm{R}}}{Q_{\mathrm{R}}}+\omega_{\mathrm{R}}^{2}}
\end{aligned}
$$

with

$$
\begin{aligned}
& \omega_{\mathrm{R}}=\sqrt{\frac{g_{1} g_{\mathrm{L}}+G_{\mathrm{M} 1} G_{\mathrm{M} 2} \cos \phi}{C_{1} C_{\mathrm{L}}}} \approx \sqrt{\frac{G_{\mathrm{M} 1} G_{\mathrm{M} 2} \cos \phi}{C_{1} C_{\mathrm{L}}}} \\
& Q_{\mathrm{R}}=\frac{\omega_{\mathrm{R}}}{\frac{g_{1}}{C_{1}}+\frac{g_{\mathrm{L}}}{C_{\mathrm{L}}}-\frac{G_{\mathrm{M} 1} G_{\mathrm{M} 2} \sin \phi\left(\omega_{\mathrm{R}}\right)}{\omega_{\mathrm{R}} C_{1} C_{\mathrm{L}}}} \approx \frac{\omega_{\mathrm{R}}}{\frac{g_{1}}{C_{1}}+\frac{g_{\mathrm{L}}}{C_{\mathrm{L}}}-\omega_{\mathrm{R}} \tan \phi\left(\omega_{\mathrm{R}}\right)}=\frac{\omega_{\mathrm{R}}}{a-b}
\end{aligned}
$$

where

$$
a=\frac{g_{1}}{C_{1}}+\frac{g_{\mathrm{L}}}{C_{\mathrm{L}}}, \quad b=\omega_{\mathrm{R}} \tan \phi\left(\omega_{\mathrm{R}}\right)
$$

The terms $a$ and $b$ represent the intrinsic circuit loss and the compensation effect through the total loop phase lag, respectively. There are a few observations:

1. As $\phi$ increases from 0 to $\leq \pi / 2, Q_{\mathrm{R}}$ will increase. By our notation, $\phi>0$ represents a phase lag.

2. The $\phi$ value near $\omega_{\mathrm{R}}$ determines $Q_{\mathrm{R}} . \phi$ values at other frequencies have no direct effect on $Q_{\mathrm{R}}$. 
3. Only the total phase lag $\phi=\phi_{1}+\phi_{2}$ matters. The $\phi$ distribution between $G_{\mathrm{M} 1}$ and $G_{\mathrm{M} 2}$ does not matter.

4. $\phi$ compensates both $g_{1}$ and $g_{\mathrm{L}}$ for $Q_{\mathrm{R}}$.

When

$$
\phi\left(\omega_{\mathrm{R}}\right) \geq \sin ^{-1} \frac{\left(\frac{g_{1}}{C_{1}}+\frac{g_{\mathrm{L}}}{C_{\mathrm{L}}}\right) \omega_{\mathrm{R}} C_{1} C_{\mathrm{L}}}{G_{\mathrm{M} 1} G_{\mathrm{M} 2}}
$$

$Q_{\mathrm{R}}$ will be $\infty$ or negative, an inadvisable situation, as the implemented circuit will be unstable and self oscillate. Nonetheless, this indicates that $\phi$ can fully compensate losses contributed by $g_{1}$ and $g_{\mathrm{L}}$ at $\omega_{\mathrm{R}}$.

The phase lag in the gyrator loop can be created through the use of a cascode transistor, a resistor to create $R-C$ delay, or a tunable capacitor. These methods are discussed in the survey of active inductors above.

\subsection{4 $Q_{\mathrm{R}}$ Sensitivities}

Using Eq. (2.37) we can calculate the $Q_{\mathrm{R}}$ sensitivities with respect to $a$ and $b$ as

$$
\begin{gathered}
S_{a}^{Q_{\mathrm{R}}}=\frac{a}{Q_{\mathrm{R}}} \frac{\partial Q_{\mathrm{R}}}{\partial a}=-Q_{\mathrm{R}} \frac{a}{\omega_{\mathrm{R}}} \\
S_{b}^{Q_{\mathrm{R}}}=\frac{b}{Q_{\mathrm{R}}} \frac{\partial Q_{\mathrm{R}}}{\partial b}=Q_{\mathrm{R}} \frac{b}{\omega_{\mathrm{R}}}
\end{gathered}
$$

which are both proportional to $Q_{\mathrm{R}}$, and restrict the maximum realizable $Q_{\mathrm{R}}$ in practice. $a$ and $b$ are both related to circuit biasing and temperature. If $Q_{\mathrm{R}}$ is designed overly high, e.g., at 400, it is going to be highly sensitive to circuit variations and disturbances, and a very small change may push $Q_{\mathrm{R}}$ to thousands or negative 
(unstable), or down to 200s. This is not surprising, since a high $Q_{\mathrm{R}}$ is derived from a delicate and measured cancellation between the effects of intrinsic circuit losses and $Q_{\mathrm{R}}$ compensation, that is, $Q_{\mathrm{R}}=1 /\left(\operatorname{large}^{1}\right.$ number - large number $)$. When the two numbers are close, a small error in either will dramatically change $Q_{\mathrm{R}}$. This is the well-known problem entailed by setting a critical circuit parameter, here $Q$, via the measured coordination of different effects. If the parameter is set by the coordination of the same effect, for example, $Q$ by a capacitor ratio, then the parameter will be very insensitive to circuit errors [10].

\subsection{Noise of Gyrator-Based Active Inductors}

Inevitably, all active inductors are noisy, since they are build with transistors (and resistors in some circuits), which generate noise. In most cases, noise is an undesirable attribute, and we would like to understand the generation mechanism and the fundamental limits, and reduce it if possible. The noise of active inductors can be represented as an equivalent shunt noise current, $i_{\mathrm{n}}{ }^{2}$ in Fig. 2-25. The noise current $i_{\mathrm{n}}{ }^{2}$ has a unit of $A^{2} / \mathrm{Hz}$, and is generally a function of frequency, i.e., $i_{\mathrm{n}}^{2}(f)$. This is sufficient in the noise analysis of typical active inductor applications, limiting/lownoise amplifiers, oscillators, and bandpass filters, and is more convenient than a series noise voltage, since in these applications, the active inductor is used as a shunt element rather than a series element.

\footnotetext{
1 "Large" is relative to the difference between the two numbers.
} 


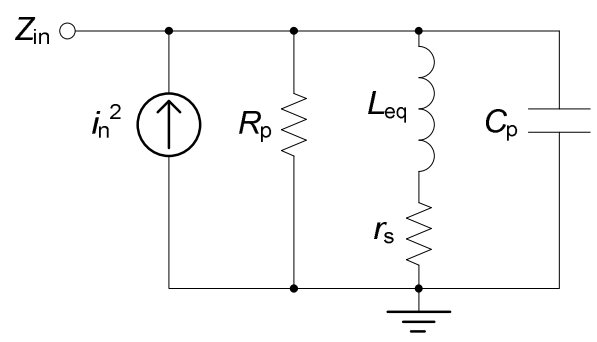

Figure 2-25 The noise model of an active inductor.

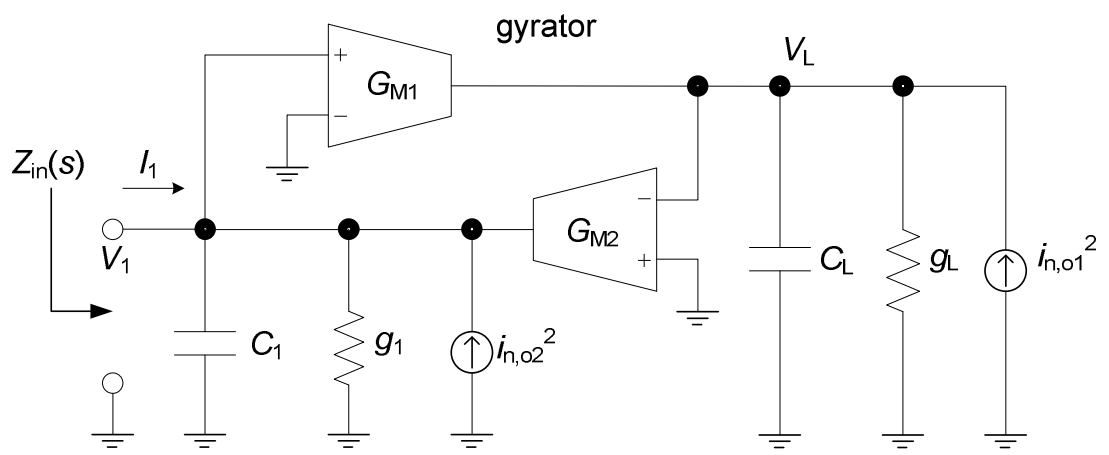

Figure 2-26 A gyrator-based active inductor showing noise elements.

The active inductor based on a gyrator is shown again in Fig. 2-26, including the noise elements from the transconductors. The noise of transconductors can be modeled as an equivalent input noise voltage, $v_{\mathrm{n}, \mathrm{i}}{ }^{2}\left(\mathrm{~V}^{2} / \mathrm{Hz}\right)$, in series with the input port or an equivalent shunt noise current, $i_{\mathrm{n}, \mathrm{o}}{ }^{2}$, at the output, and they are related by

$$
i_{\mathrm{n}, \mathrm{o}}^{2}=G_{\mathrm{M}}^{2} v_{\mathrm{n}, \mathrm{i}}^{2}
$$

The noise voltage $v_{n, i}{ }^{2}$ has a unit of $\mathrm{V}^{2} / \mathrm{Hz}$, and generally is a function of frequency, too. We choose to use the equivalent current. A higher value $G_{\mathrm{M}}$ will have higher noise level; however, a complicated circuit structure may also yield a higher noise level. A measure of transconductor noise level is herein defined as noise-factor coefficient, by dividing its squared noise to that of a resistor of equal value,

$$
K_{\mathrm{NF}}=\frac{i_{\mathrm{n}, \mathrm{o}}^{2}}{4 k T G_{\mathrm{M}}}
$$


It can be shown that $K_{\mathrm{NF}}$ is unitless, and for a resistor, $K_{\mathrm{NF}}=1$. Note that a high $K_{\mathrm{NF}}$ may be a byproduct of other desirable features, such as high linearity. The conductors $g_{1}$ and $g_{\mathrm{L}}$ represent transconductor output losses; they are not physical resistors and their noise contributions have been included in $i_{\mathrm{n}, 01}$ and $i_{\mathrm{n}, 02}$ (both in $\mathrm{A} / \mathrm{Hz}^{1 / 2}$ ), hence their noises are not shown separately in the circuit.

Next we use node equations to derive the equivalent noise of the active inductor. From the node equation at $V_{\mathrm{L}}$,

$$
V_{\mathrm{L}}\left(s C_{\mathrm{L}}+g_{\mathrm{L}}\right)=G_{\mathrm{M} 1} V_{1}+i_{\mathrm{n}, 01}
$$

we have

$$
V_{\mathrm{L}}=\frac{G_{\mathrm{M} 1}}{s C_{\mathrm{L}}+g_{\mathrm{L}}} V_{1}+\frac{i_{\mathrm{n}, 01}}{s C_{\mathrm{L}}+g_{\mathrm{L}}}
$$

Note that the node voltages $V_{\mathrm{L}}$ and $V_{1}$ are actually noise voltages, since we are using node equations for noise analysis, and have a unit of $\mathrm{V} / \mathrm{Hz}^{1 / 2}$. The same is true for the voltage and current signals in other noise analysis equations. Plugging this into the node equation at $V_{1}$,

$$
V_{1}\left(s C_{1}+g_{1}\right)+G_{\mathrm{M} 2} V_{\mathrm{L}}=i_{\mathrm{n}, 02}
$$

we have

$$
V_{1}\left(s C_{1}+g_{1}\right)+\frac{1}{\left(s C_{\mathrm{L}}+g_{\mathrm{L}}\right) /\left(G_{\mathrm{M} 1} G_{\mathrm{M} 2}\right)} V_{1}+\frac{G_{\mathrm{M} 2}}{s C_{\mathrm{L}}+g_{\mathrm{L}}} i_{\mathrm{n}, 01}=i_{\mathrm{n}, 02}
$$

where $\left(s C_{\mathrm{L}}+g_{\mathrm{L}}\right) /\left(G_{\mathrm{M} 1} G_{\mathrm{M} 2}\right)$ is recognized as the lossy inductor converted from the load by the gyrator, and the third term is an equivalent shunt noise current. In other words, the effect of noise source $i_{\mathrm{n}, 01}$ can be moved to the output of $G_{\mathrm{M} 2}$ by a transfer 
function, despite the existence of a feedback loop formed by $G_{\mathrm{M} 1}$ and $G_{\mathrm{M} 2}$. Thus, the total equivalent noise current in shunt with the input port of the active inductor is

$$
i_{\mathrm{n}}^{2}=i_{\mathrm{n}, 02}^{2}+\frac{G_{\mathrm{M} 2}^{2}}{\omega^{2} C_{\mathrm{L}}^{2}+g_{\mathrm{L}}^{2}} i_{\mathrm{n}, \mathrm{o} 1}^{2}=i_{\mathrm{n}, 02}^{2}+\left|T_{\mathrm{N} 1}(\omega)\right|^{2} i_{\mathrm{n}, 01}^{2}
$$

where $T_{\mathrm{N} 1}(\omega)$ is the noise transfer function for $i_{\mathrm{n}, \mathrm{o} 1}$.

Therefore, the noise contribution from $i_{\mathrm{n}, \mathrm{o} 1}$ has been shaped by a low pass transfer function. As a special but representative case, consider $G_{\mathrm{M} 1}=G_{\mathrm{M} 2}, C_{1}=C_{\mathrm{L}}$, and ignore the effects of $g_{1}$ and $g_{\mathrm{L}}$, at the frequency $\omega=\omega_{\mathrm{R}}=G_{\mathrm{M} 1} / C_{1}$, we have

$$
i_{\mathrm{n}}^{2}=i_{\mathrm{n}, 02}^{2}+\frac{G_{\mathrm{M} 1}^{2}}{\left(G_{\mathrm{M} 1} / C_{1}\right)^{2} C_{1}^{2}+0} i_{\mathrm{n}, \mathrm{o} 1}^{2}=i_{\mathrm{n}, 02}^{2}+1 \cdot i_{\mathrm{n}, \mathrm{o} 1}^{2}
$$

i.e., the noise transfer function for $i_{\mathrm{n}, \mathrm{o} 1}$ is 1 . For frequencies $\omega<\omega_{\mathrm{R}}$, the magnitude of the noise transfer function for $i_{\mathrm{n}, \mathrm{o} 1}$ will be greater than 1 :

$$
\left|T_{\mathrm{N} 1}(\omega)\right|^{2}=\frac{G_{\mathrm{M} 2}^{2}}{\omega^{2} C_{\mathrm{L}}^{2}+g_{\mathrm{L}}^{2}} \approx \frac{G_{\mathrm{M} 2}^{2} / C_{\mathrm{L}}^{2}}{\omega^{2}} \approx \frac{\omega_{\mathrm{R}}^{2}}{\omega^{2}}>1
$$

Using the definition of Eq. (2.42), we have

$$
i_{\mathrm{n}}^{2}=i_{\mathrm{n}, 02}^{2}+\frac{\omega_{\mathrm{R}}^{2}}{\omega^{2}} i_{\mathrm{n}, 01}^{2}=4 k T\left(K_{\mathrm{NF} 2} G_{\mathrm{M} 2}+\frac{\omega_{\mathrm{R}}^{2}}{\omega^{2}} K_{\mathrm{NF} 1} G_{\mathrm{M} 1}\right)
$$

Therefore, for a given $\omega_{\mathrm{R}}$ and $\omega\left(<\omega_{\mathrm{R}}\right)$, to reduce overall equivalent noise, we should reduce $i_{\mathrm{n}, \mathrm{o} 1}$; whether this means $G_{\mathrm{M} 1}$ should be less than $G_{\mathrm{M} 2}$ depends on the $G_{\mathrm{M}}$ structure's noise factors $K_{\mathrm{NF} 1}$ and $K_{\mathrm{NF} 2}$. Note that for practical circuits, the $G_{\mathrm{M} 1}$ and $G_{\mathrm{M} 2}$ values cannot be too different, for example, more than an order of magnitude, because the parasitic effects (e.g. $C_{\mathrm{in}}, C_{\mathrm{out}}$, phase shift) of the larger $G_{\mathrm{M}}$ cell can overwhelm the smaller $G_{\mathrm{M}}$ cell. 
Similarly, in the case of $K_{\mathrm{NF} 1}=K_{\mathrm{NF} 2}$, we cannot make $G_{\mathrm{M} 1}=0$ to minimize the overall noise. In other words, depending on the particular circuits, a $G_{\mathrm{M} 1}$ to $G_{\mathrm{M} 2}$ ratio optimum for low noise is likely to be outside of practicality.

Per Fig. 2-26, when the active inductor port is floating, the equivalent output noise voltage, $v_{\mathrm{n}, \mathrm{o}}\left(\mathrm{V} / \mathrm{Hz}^{1 / 2}\right)$, on the port will be

$$
v_{\mathrm{n}, \mathrm{o}}^{2}(\omega)=\left|Z_{\mathrm{in}}(\omega)\right|^{2} i_{\mathrm{n}, \mathrm{o}}^{2}(\omega) \text { or } v_{\mathrm{n}, \mathrm{o}}(\omega)=\left|Z_{\mathrm{in}}(\omega)\right| i_{\mathrm{n}, \mathrm{o}}(\omega)
$$

which is the equivalent shunt noise current shaped by the active inductor $Z_{\text {in }}$ profile.

\subsection{The All-NMOS Active Inductor (New in This Work)}

\subsubsection{The All-NMOS Active-Inductor Circuit}

This circuit is developed from Karsilayan's circuit (Fig. 2-23) and is shown in Fig. 227 [80-82]. Its $Z_{\text {in }}$ is equivalent to that of a grounded inductor. It is new in this work, and the main difference from Karsilayan's circuit is that the noninverting $G_{\mathrm{M}}$ is implemented using an NMOS differential pair rather than a PMOS differential pair. The inverting $G_{\mathrm{M}}$ is not changed. NFETs have higher mobility than PFETs and thus the active inductor has higher self-resonance frequency $f_{\mathrm{R}}$ due to its all-NMOS signal path. The frequency improvement is about $30 \%^{2}$.

\footnotetext{
${ }^{2} f_{\mathrm{R}}$ of the all-NMOS active inductor is measured to be $5.7 \mathrm{GHz}$ at $0.2-\mu \mathrm{m}$ gate length, while in Karsilayan's circuit $f_{\mathrm{R}}$ was measured to be $4.1 \mathrm{GHz}$ for the same technology and gate length. Results of the latter are unpublished.
} 


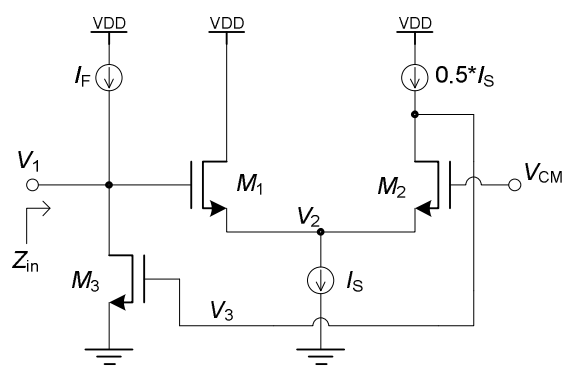

(a)

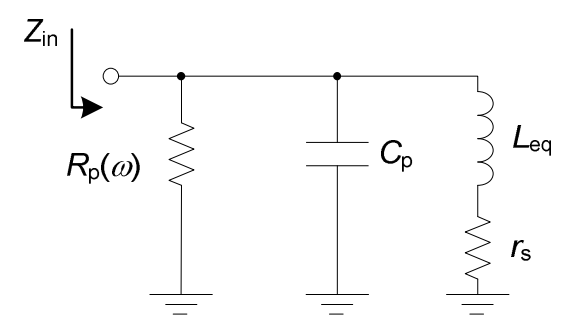

(b)

Figure 2-27 (a) The all-NMOS signal-path active-inductor circuit. (b) Equivalent circuit.

The circuit's operation can be understood by tracing the signal flow. Transistors $M_{1}$ and $M_{2}$ implement a noninverting $G_{\mathrm{M}}$, labeled $G_{\mathrm{M} 1}\left(=0.5 g_{\mathrm{m} 1}\right.$, if $g_{\mathrm{m} 1}=g_{\mathrm{m} 2}$ and the loss at node $2, g_{2}$, is zero), with input voltage $V_{1}$ and output current at $V_{3} . M_{3}$ is an inverting transconductor, labeled $-G_{\mathrm{M} 2}\left(=-g_{\mathrm{m} 3}\right)$, with input voltage $V_{3}$ and output current at $V_{1}$. Thus, $G_{\mathrm{M} 1}$ and $-G_{\mathrm{M} 2}$ form a gyrator, and convert the parasitic capacitor $C_{3}$ at Node 3 into a grounded inductor, $L_{\mathrm{eq}}=C_{3} /\left(G_{\mathrm{M} 1} G_{\mathrm{M} 2}\right)$, at node 1 . The signal path consists of only NMOS transistors to take advantage of their higher mobility for the same device geometry. Therefore, the circuit can operate at a higher frequency [80, 81] than those built with a CMOS signal path [65-67]. The minimum $V_{\mathrm{DD}}$ is $V_{\mathrm{DS}, \mathrm{p}}+$ $V_{\mathrm{GS}, \mathrm{n}}+V_{\mathrm{DS}, \mathrm{n}}$, which is around $1 \mathrm{~V}$ depending on the IC process and biasing conditions. For comparison, the minimum $V_{\mathrm{DD}}$ requirement of Karsilayan's circuit is $V_{\mathrm{DS}, \mathrm{p}}+V_{\mathrm{GS}, \mathrm{p}}$ $+V_{\mathrm{DS}, \mathrm{n}}$. The difference in $V_{\mathrm{DD}}$ requirement of the two circuit is $V_{\mathrm{T}, \mathrm{n}}-V_{\mathrm{T}, \mathrm{p}}$, which is determined by the process, when the transistors have the same overdrive voltages. 


\subsubsection{Small-Signal Analysis}

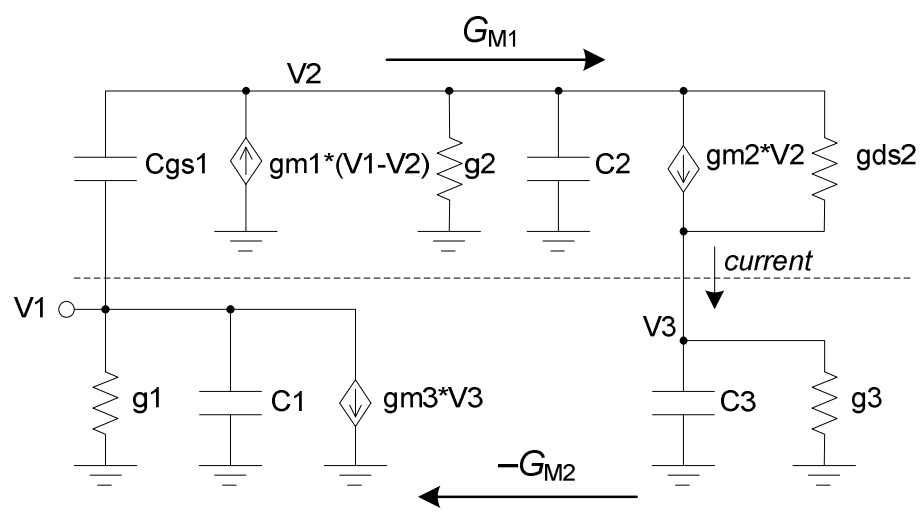

Figure 2-28 Small-signal equivalent circuit of the active inductor in Fig. 2-27(a). $g_{\mathrm{m} 1-3}$ are the transconductances of $M_{1-3}$, and $C_{1-3}$ and $g_{1-3}$ are the total parasitic capacitances/conductances at nodes 1-3, respectively. $C_{2}$ also includes the capacitance from a small varactor for $Q$ tuning.

The input impedance $Z_{\text {in }}$ can be derived from an analysis of the small-signal ac equivalent circuit in Fig. 2-28. A mathematically accurate expression including all terms can of course be obtained; however, to provide designers with better insight, a few small parasitic terms are neglected when summed with dominant terms. The approximate expression is

$$
Z_{\text {in }}(s) \approx \frac{\frac{s}{C_{1}}+\frac{g_{3}}{C_{1} C_{3}}}{s^{2}+s\left(\frac{g_{1}}{C_{1}}+\frac{g_{3}}{C_{3}}+\frac{g_{1} g_{3} C_{2}}{C_{1} C_{3} G}-\omega^{2} \frac{C_{2}}{G}\right)+\frac{g_{\mathrm{m} 2}}{G} \frac{g_{\mathrm{m} 1} g_{\mathrm{m} 3}}{C_{1} C_{3}}}
$$

where $G=g_{\mathrm{m} 1}+g_{\mathrm{m} 2}+g_{2}$, and the equivalence $s^{3}=-\omega^{2} s$ has been used, because the effect of the negative real pole from the $s^{3}$ term is not dominant, compared with that of the dominant conjugate complex poles. The format of $Z_{\text {in }}$ shows that it is equivalent to an $R L C$ network, as shown in Fig. 2-27(b): the denominator together with the $s$ term in the numerator indicates an $L C$ resonance with limited $Q$, and the real term in the numerator indicates a resistor in series with the inductor. The element values can be determined from $Z_{\text {in }}$ as follows. At dc $(s=0)$, 


$$
\begin{aligned}
& Z_{\text {in }}(0)=\frac{g_{3} G}{g_{\mathrm{m} 1} g_{\mathrm{m} 2} g_{\mathrm{m} 3}}=\frac{g_{3}\left(g_{\mathrm{m} 1}+g_{\mathrm{m} 2}+g_{2}\right)}{g_{\mathrm{m} 1} g_{\mathrm{m} 2} g_{\mathrm{m} 3}}=\frac{g_{3}\left(g_{\mathrm{m} 1} / g_{\mathrm{m} 2}+1+g_{2} / g_{\mathrm{m} 2}\right)}{g_{\mathrm{m} 1} g_{\mathrm{m} 3}} \\
& \approx \frac{g_{3}}{0.5 g_{\mathrm{m} 1} g_{\mathrm{m} 3}}=r_{\mathrm{s}} \text {, when } g_{\mathrm{m} 1} \approx g_{\mathrm{m} 2} \text { and } g_{2}<<g_{\mathrm{m} 1}, g_{\mathrm{m} 2}
\end{aligned}
$$

where $r_{\mathrm{s}}$ is the resistor in series with $L_{\mathrm{eq}}$; it dominates $R_{\mathrm{p}}(\omega)$ at dc. At very high frequencies, $Z_{\text {in }} \propto 1 /\left(s C_{1}\right)$, hence the parallel capacitor is

$$
C_{\mathrm{p}}=C_{1}
$$

The resonance frequency is

$$
\omega_{\mathrm{R}}=\sqrt{\frac{g_{\mathrm{m} 1} g_{\mathrm{m} 2} g_{\mathrm{m} 3}}{G C_{1} C_{3}}} \approx \sqrt{\frac{0.5 g_{\mathrm{m} 1} g_{\mathrm{m} 3}}{C_{1} C_{3}}}=\frac{1}{\sqrt{L_{\mathrm{eq}} C_{1}}}
$$

At intermediate frequencies between $g_{3} / C_{3}$ and $\omega_{\mathrm{R}}, Z_{\text {in }}(s)$ can be transformed into

$$
\begin{aligned}
& Z_{\text {in }}(s)=\frac{\frac{s G C_{3}}{g_{\mathrm{m} 1} g_{\mathrm{m} 2} g_{\mathrm{m} 3}}\left(1+\frac{g_{3} / C_{3}}{s}\right)}{\frac{s^{2}}{\omega_{\mathrm{R}}^{2}}+\frac{s}{\omega_{\mathrm{R}}^{2}}\left(\frac{g_{1}}{C_{1}}+\frac{g_{3}}{C_{3}}+\frac{g_{1} g_{3} C_{2}}{C_{1} C_{3} G}-\omega^{2} \frac{C_{2}}{G}\right)+1} \approx \frac{\frac{s G C_{3}}{g_{\mathrm{m} 1} g_{\mathrm{m} 2} g_{\mathrm{m} 3}}}{0+0+1}(1+0) \\
& =s \frac{G C_{3}}{g_{\mathrm{m} 1} g_{\mathrm{m} 2} g_{\mathrm{m} 3}}=s L_{\mathrm{eq}}, \quad \text { when } \frac{g_{3}}{C_{3}}<<|s|<<\omega_{\mathrm{R}}
\end{aligned}
$$

which represents an inductor. $L_{\mathrm{eq}}$ can also be derived from Eqs. (2.54) and (2.55),

$$
L_{\mathrm{eq}}=\frac{G C_{3}}{g_{\mathrm{m} 1} g_{\mathrm{m} 2} g_{\mathrm{m} 3}} \approx \frac{C_{3}}{0.5 g_{\mathrm{m} 1} g_{\mathrm{m} 3}} \text {, when } g_{\mathrm{m} 1} \approx g_{\mathrm{m} 2} \text { and } g_{2}<<g_{\mathrm{m} 1}, g_{\mathrm{m} 2}
$$

The quality factor at $\omega_{\mathrm{R}}$ is

$$
Q_{\mathrm{R}}=Q\left(\omega_{\mathrm{R}}\right)=\frac{\sqrt{g_{\mathrm{m} 1} g_{\mathrm{m} 2} g_{\mathrm{m} 3} /\left(G C_{1} C_{3}\right)}}{\frac{g_{1}}{C_{1}}+\frac{g_{3}}{C_{3}}-\frac{C_{2}}{G}\left(\omega_{\mathrm{R}}^{2}-\frac{g_{1} g_{3}}{C_{1} C_{3}}\right)}=\frac{\omega_{\mathrm{R}}}{a-b}
$$

where $a$ and $b$ are 


$$
a=\frac{g_{1}}{C_{1}}+\frac{g_{3}}{C_{3}}, b=\frac{C_{2}}{G}\left(\omega_{\mathrm{R}}^{2}-\frac{g_{1} g_{3}}{C_{1} C_{3}}\right)
$$

$a$ represents again the intrinsic loss of the circuit and $b$ represents the compensation effected through $C_{2}$. $C_{2}$ is at the internal node of the noninverting $G_{\mathrm{M}}$. A larger $C_{2}$ will yield a larger phase lag $\phi$ in the noninverting $G_{\mathrm{M}}$ and hence a larger $Q_{\mathrm{R}}$, per Eq. (2.37). Since $g_{i}<<g_{\mathrm{m} i}, i=1,3$, we always have

$$
\omega_{\mathrm{R}}^{2}-\frac{g_{1} g_{3}}{C_{1} C_{3}}=\frac{1}{C_{1} C_{3}}\left(\frac{g_{\mathrm{m} 1} g_{\mathrm{m} 2} g_{\mathrm{m} 3}}{G}-g_{1} g_{3}\right) \approx \frac{1}{C_{1} C_{3}}\left(\frac{g_{\mathrm{m} 1} g_{\mathrm{m} 3}}{2}-g_{1} g_{3}\right)>0
$$

hence the loss compensation through $C_{2}$ is guaranteed. When

$$
C_{2}=\left(\frac{g_{1}}{C_{1}}+\frac{g_{3}}{C_{3}}\right) \frac{G}{\omega_{\mathrm{R}}^{2}-g_{1} g_{3} /\left(C_{1} C_{3}\right)} \approx\left(\frac{g_{1}}{C_{1}}+\frac{g_{3}}{C_{3}}\right) \frac{G}{\omega_{\mathrm{R}}^{2}}
$$

$Q_{\mathrm{R}}$ will be infinite. Although $Q_{\mathrm{R}}=\infty$ is not advisable in practice since the circuit will oscillate, it nevertheless indicates that there is no upper limit on $Q_{\mathrm{R}}$ with this activeinductor structure because $C_{2}$ can compensate the effects of $g_{1}, g_{2}$, and $g_{3}$, that is, of all losses in the circuit, around $f_{\mathrm{R}}$. But observe the potentially huge sensitivities in Eq. (2.40) as $Q_{\mathrm{R}}$ increases.

The shunt equivalent resistor $R_{\mathrm{p}}$ can be derived from Eq. (2.8),

$$
R_{\mathrm{p}}=Q_{\mathrm{R}} \sqrt{\frac{L_{\mathrm{eq}}}{C_{\mathrm{p}}}}=\frac{1}{g_{1}+g_{3} \frac{C_{1}}{C_{3}}-\frac{C_{1} C_{2}}{G}\left(\omega_{\mathrm{R}}^{2}-\frac{g_{1} g_{3}}{C_{1} C_{3}}\right)}
$$




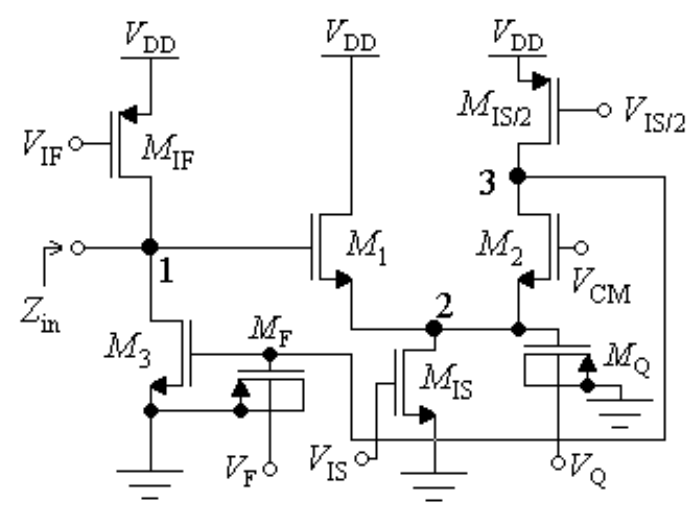

(a)

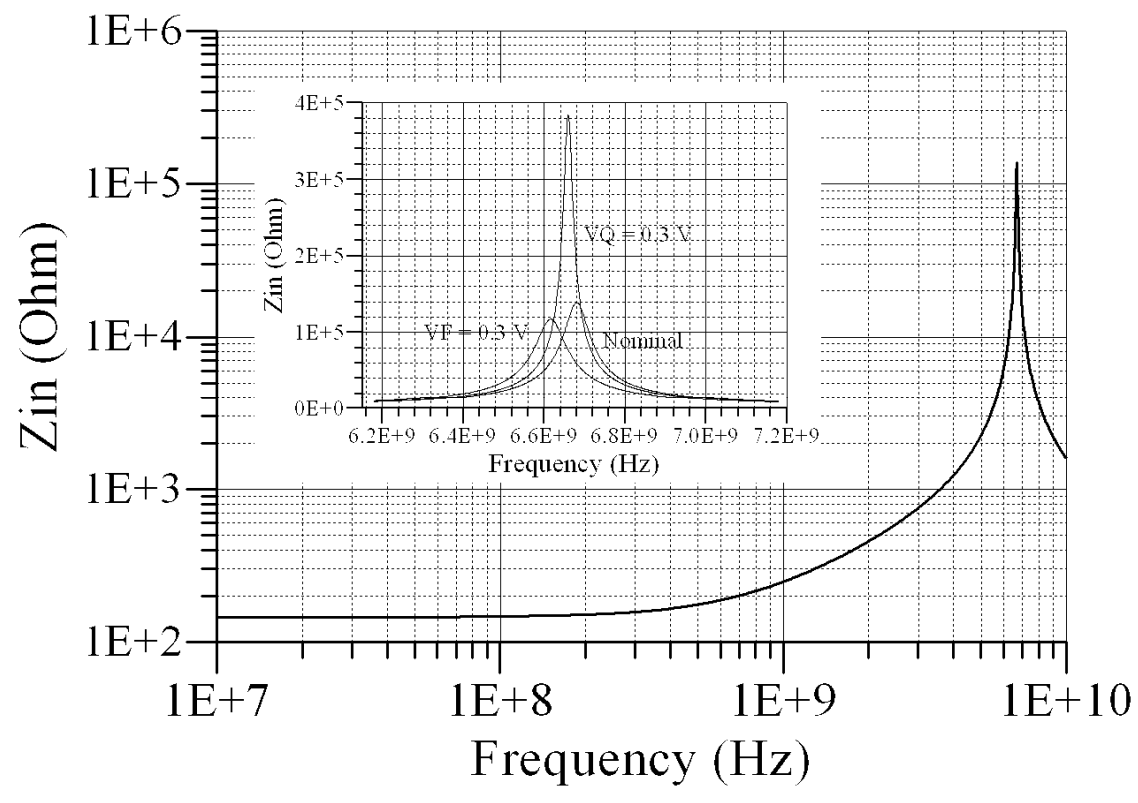

(b)

Figure 2-29 Example simulations of the all-NMOS signal-path active inductor in a pseudo-differential structure. (a) The active inductor circuit with biasing and tuning components shown. (b) The magnitude of $Z_{\text {in }}$ as simulated in the TSMC $0.18-\mu \mathrm{m}$ standard digital CMOS process. Transistor sizes $(\mathrm{W} / \mathrm{L}$ in $\mu \mathrm{m})$ and nominal bias values are: $M_{1,2,3} 3 / 0.2, M_{\mathrm{IF}, \mathrm{IS} / 2} 6 / 0.2, M_{\mathrm{IS}} 8.4 / 0.2, M_{\mathrm{F}}$ $1.5 / 0.4, M_{\mathrm{Q}} 3 / 0.4, I_{\mathrm{M}_{\mathrm{IF}}}=I_{\mathrm{M}_{\mathrm{IS}}}=250 \mu \mathrm{A}, V_{\mathrm{F}}=V_{\mathrm{Q}}=0.6 \mathrm{~V}, V_{\mathrm{CM}}=1.325 \mathrm{~V}, V_{\mathrm{DD}}=1.8 \mathrm{~V}, f_{\mathrm{R}}=6.68$ $\mathrm{GHz}, Q_{\mathrm{R}}=106$. Shown in the insert are the ("Nominal") plot of $Z_{\text {in }}$ "zoomed-in" around $f_{\mathrm{R}}$ at the above biasing conditions, and two others showing $f_{\mathrm{R}}$ and $Q_{\mathrm{R}}$ tuning. When $V_{\mathrm{F}}$ is reduced to 0.3 $\mathrm{V}, f_{\mathrm{R}}=6.613 \mathrm{GHz}$, and $Q_{\mathrm{R}}$ becomes 87 ; when $V_{\mathrm{Q}}$ is reduced to $0.3 \mathrm{~V}, Q_{\mathrm{R}}=289$ and $f_{\mathrm{R}}$ becomes $6.658 \mathrm{GHz}$. Other conditions remain the same in both cases.

The $Q_{\mathrm{R}}$ enhancement and tuning are achieved solely through the capacitor $C_{2}$ (tuned by the varactor $M_{\mathrm{Q}}$, see Fig. 2-29), whose major portion is already in the circuit as a parasitic component; no additional loss-compensation circuitry, such as negative 
resistors, is used. Hence, there is no power and only a small frequency penalty ${ }^{3}$ for obtaining a large $Q_{\mathrm{R}}$, and the circuit structure is very concise and efficient.

The complete circuit is shown in Fig. 2-29(a) and some representative simulation results are contained in Fig. 2-29(b). Two varactors, $M_{\mathrm{F}}$ and $M_{\mathrm{Q}}$, are added for tuning $f_{\mathrm{R}}$ and $Q_{\mathrm{R}}$, respectively. Tuning of $f_{\mathrm{R}}$ and $Q_{\mathrm{R}}$ via varactors is relatively independent within a moderate tuning range; hence there will be little or no need for tuning iterations. Note that single-transistor current sources can be used because their higher output losses are readily compensated by the choice of $C_{2}$. This saves devices, power and makes the active inductor/resonator suitable for low-voltage designs. As seen from Eqs. (2.55) and (2.58), $f_{\mathrm{R}}$ and $Q_{\mathrm{R}}$ are also tunable via the bias currents $I_{\mathrm{F}}$ and $I_{\mathrm{S}}$ over a wider range, since they change the values of $g_{\mathrm{m}}$ and $g_{\mathrm{ds}}$.

To verify the equations, the circuit was simulated. As shown in Fig. 2-29(b), below $40 \mathrm{MHz}, Z_{\text {in }}$ is roughly equal to a $146-\Omega$ resistor; between $500 \mathrm{MHz}$ and $4 \mathrm{GHz}$, $\left|Z_{\text {in }}\right| \propto \omega$ and is equivalent to an inductor of $31 \mathrm{nH}$ in series with the $146-\Omega$ resistor; above $12 \mathrm{GHz},\left|Z_{\text {in }}\right| \propto 1 / \omega$ : it behaves like a capacitor of $16.8 \mathrm{fF}$. All frequency ranges are approximate as there is no clear cutoff between the regions. Its resonance frequency is $f_{\mathrm{R}}=6.68 \mathrm{GHz}$, and $Q_{\mathrm{R}}=106$.

The small-signal parameters, $g_{\mathrm{m} 1}=1.15 \mathrm{mS}, g_{\mathrm{m} 2}=1.14 \mathrm{mS}, g_{\mathrm{m} 3}=1.15 \mathrm{mS}, C_{1}=$ $16.8 \mathrm{fF}, C_{2}=16.4 \mathrm{fF}, C_{3}=20.4 \mathrm{fF}, g_{1}=132 \mu \mathrm{S}, g_{3}=91 \mu \mathrm{S}$, and $G=2.44 \mathrm{mS}$ are found from the operating points. Using these parameters in the equations, the circuit

\footnotetext{
${ }^{3}$ Unlike $C_{1}$ and $C_{3}, C_{2}$ has only a second-order effect on $f_{\mathrm{R}}$, as shown by Eq. (2.55). However, as expected, increasing $C_{2}$ does reduce $f_{\mathrm{R}}$ by a relatively small amount due to second- and higher-order effects through the parasitic elements in the equivalent circuit (Fig. 2.28).
} 
parameters, $L_{\mathrm{eq}}, r_{\mathrm{s}}, f_{\mathrm{R}}, Q_{\mathrm{R}}$ and $R_{\mathrm{p}}$ can be calculated, as shown in Table 2-1. The calculated parameters are very close to the simulation results. Nevertheless, it is noted again that due to the potential high circuit sensitivities when $Q_{\mathrm{R}}$ is high and the approximation made in the small-signal circuit, the equations are not given for obtaining precise circuit performance, but to provide insight into the circuit's operation and to facilitate an informed design process. More accurate circuit performance in design should be obtained from circuit simulations, based on accurate device modeling and layout parasitics extraction.

Table 2-1 Calculated versus Simulated Results for the All-NMOS Active Inductor

\begin{tabular}{|l|l|r|l|l|}
\hline Parameter & Unit & \multicolumn{1}{l|}{ Sims } & \multicolumn{1}{l|}{ Calc'ed } & Equation \\
\hline$g_{\mathrm{m} 1}$ & $\mathrm{mS}$ & 1.15 & & \\
\hline$g_{\mathrm{m} 2}$ & $\mathrm{mS}$ & 1.14 & & \\
\hline$g_{\mathrm{m} 3}$ & $\mathrm{mS}$ & 1.15 & & \\
\hline$C_{1}$ & $\mathrm{fF}$ & 16.8 & & \\
\hline$C_{2}$ & $\mathrm{fF}$ & 16.4 & & \\
\hline$C_{3}$ & $\mathrm{fF}$ & 20.4 & & \\
\hline$g_{1}$ & $\mathrm{uS}$ & 132 & & \\
\hline$g_{3}$ & $\mathrm{uS}$ & 91 & & \\
\hline$G$ & $\mathrm{mS}$ & 2.44 & & \\
\hline & & & & \\
\hline$L_{\mathrm{eq}}$ & $\mathrm{nH}$ & $\mathbf{3 1}$ & $\mathbf{3 3 . 0}$ & $(2.57)$ \\
\hline$r_{\mathrm{s}}$ & $\mathrm{Ohm}$ & $\mathbf{1 4 6}$ & $\mathbf{1 4 7 . 3}$ & $(2.53)$ \\
\hline$f_{\mathrm{R}}$ & $\mathrm{GHz}$ & $\mathbf{6 . 6 8}$ & $\mathbf{6 . 7 6}$ & $(2.55)$ \\
\hline$\omega_{\mathrm{R}}$ & $\mathrm{Grad} / \mathrm{s}$ & & 42.5 & $(2.55)$ \\
\hline$Q_{\mathrm{R}}$ & & $\mathbf{1 0 6}$ & $\mathbf{9 7}$ & $(2.58)$ \\
\hline$R_{\mathrm{p}}$ & $\mathrm{kOhm}$ & $\mathbf{1 4 0}$ & $\mathbf{1 3 7}$ & $(2.62)$ \\
\hline
\end{tabular}

\subsubsection{Noise Analysis}

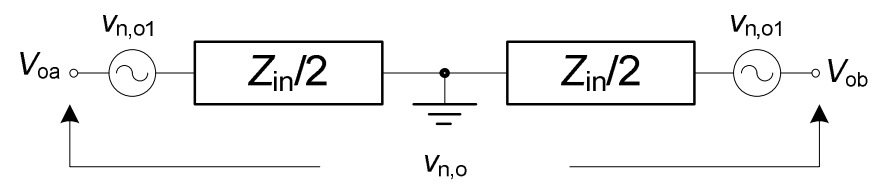

Figure 2-30 The noise model of a pseudo-differential active inductor. 
The noise model of the pseudo-differential inductor is shown in Fig. 2-30. From

$$
v_{\mathrm{n}, \mathrm{o}}^{2}=v_{\mathrm{n}, 01}^{2}+v_{\mathrm{n}, 01}^{2}=2 \cdot v_{\mathrm{n}, 01}^{2}
$$

we have

$$
v_{\mathrm{n}, \mathrm{o} 1}=\frac{v_{\mathrm{n}, \mathrm{o}}}{\sqrt{2}}
$$

Thus, the equivalent shunt noise current for each half of the active inductor is

$$
i_{\mathrm{n}, 01}=\frac{v_{\mathrm{n}, \mathrm{o} 1}}{\left|Z_{\mathrm{in}} / 2\right|}=\sqrt{2} \frac{v_{\mathrm{n}, \mathrm{o}}}{\left|Z_{\mathrm{in}}\right|}=\sqrt{2} i_{\mathrm{n}, \mathrm{o}}
$$

This is verified by circuit simulation results shown in Fig. $2-31$, as $i_{\mathrm{n}, \mathrm{o}}=10.51$ $\mathrm{pA} / \mathrm{Hz}^{1 / 2}$ at $6.68 \mathrm{GHz}$, while $\sqrt{2} i_{\mathrm{n}, \mathrm{o}}=9.73 \mathrm{pA} / \mathrm{Hz}^{1 / 2}$ per Fig. $2-31$. The difference is about $7.4 \%$.

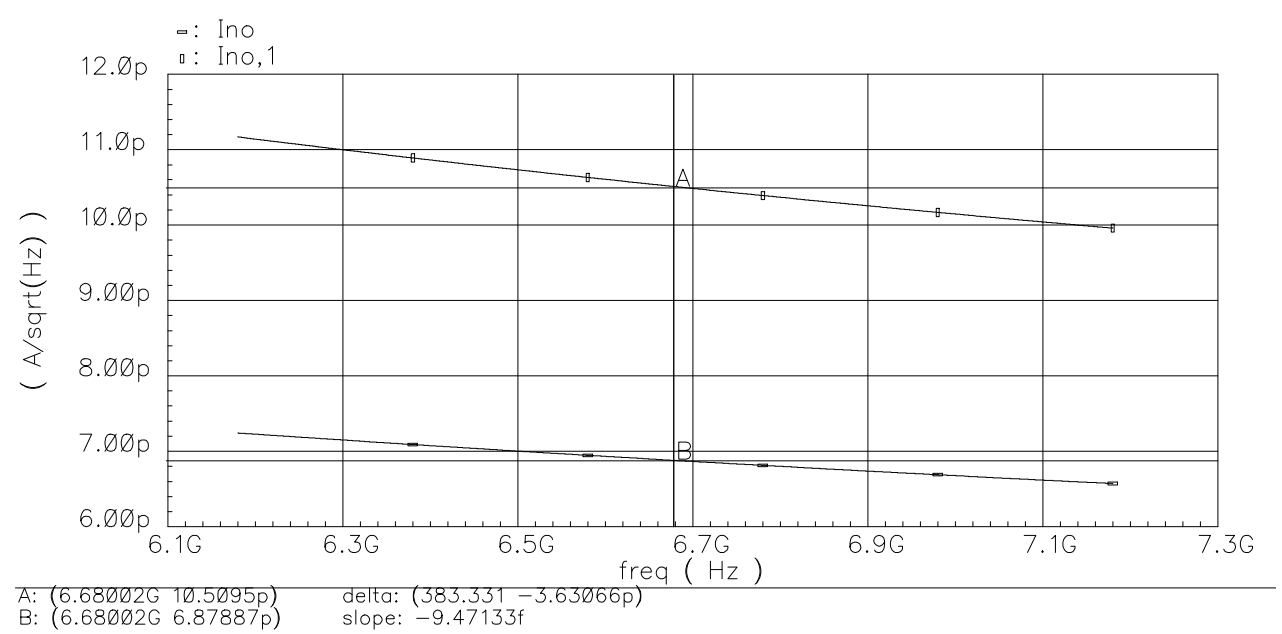

Figure 2-31 The equivalent output noise currents of the all-NMOS active inductor. The biasing conditions are the same as those in Fig. 2-29.

Next we will use the theory in Section 2.6 to derive the noise equations for the two $G_{\mathrm{M}}$ cells in the all-NMOS active inductor. For an NFET and a PFET carrying the same $I_{\mathrm{D}}$, for example, $M_{3}$ and $M_{\mathrm{IF}}$, we typically choose the similar gate overdrive voltage, to even the dynamic-range limiting and as a recommended circuit design 
practice, that is

$$
\frac{V_{\text {od,p }}}{V_{\text {od,n }}}=\sqrt{\frac{2 I_{\mathrm{D}}}{\mu_{\mathrm{p}} C_{\mathrm{ox}, \mathrm{p}}\left(\frac{W}{L}\right)_{\mathrm{p}}}} / \sqrt{\frac{2 I_{\mathrm{D}}}{\mu_{\mathrm{n}} C_{\mathrm{ox}, \mathrm{n}}\left(\frac{W}{L}\right)_{\mathrm{n}}}}=\sqrt{\frac{\mu_{\mathrm{n}} C_{\mathrm{ox, \textrm {n }}}\left(\frac{W}{L}\right)_{\mathrm{n}}}{\mu_{\mathrm{p}} C_{\mathrm{ox}, \mathrm{p}}\left(\frac{W}{L}\right)_{\mathrm{p}}}} \approx 1
$$

Thus we have

$$
\frac{g_{\mathrm{m}, \mathrm{p}}}{g_{\mathrm{m}, \mathrm{n}}}=\sqrt{\frac{2 I_{\mathrm{D}} \mu_{\mathrm{p}} C_{\mathrm{ox}, \mathrm{p}}\left(\frac{W}{L}\right)_{\mathrm{p}}}{2 I_{\mathrm{D}} \mu_{\mathrm{n}} C_{\mathrm{ox}, \mathrm{n}}\left(\frac{W}{L}\right)_{\mathrm{n}}}}=1 \text {, when } I_{\mathrm{D}, \mathrm{p}}=I_{\mathrm{D}, \mathrm{n}}
$$

Therefore, for $G_{\mathrm{M} 1}\left(M_{1}\right.$ and $M_{2}$ in Fig. 2-29(a)), assuming $g_{\mathrm{m} 1}=g_{\mathrm{m} 2}=0.5 g_{\mathrm{m}, \mathrm{IS}}$ (because they have the same $V_{\mathrm{OD}}$ ) and ignoring flicker noise and the effect of $C_{2}$ at node 2 , we have

$$
\begin{aligned}
& i_{\mathrm{n}, \mathrm{gm} 1}^{2} \approx\left(i_{\mathrm{n}, \mathrm{M} 1}^{2}+i_{\mathrm{n}, \mathrm{M} 2}^{2}+i_{\mathrm{n}, \mathrm{MIS}}^{2}\right)\left(\frac{1}{g_{\mathrm{m} 1}+g_{\mathrm{m} 2}}\right)^{2} g_{\mathrm{m} 2}^{2}+i_{\mathrm{n}, \mathrm{MIS} / 2}^{2} \\
& \approx\left[4 k T \Gamma_{\mathrm{n}} g_{\mathrm{m} 1}+4 k T \Gamma_{\mathrm{n}} g_{\mathrm{m} 2}+4 k T \Gamma_{\mathrm{n}}\left(2 g_{\mathrm{m} 2}\right)\right]\left(\frac{1}{2 g_{\mathrm{m} 2}}\right)^{2} g_{\mathrm{m} 2}^{2}+4 k T \Gamma_{\mathrm{p}} g_{\mathrm{m} 2} \\
& \approx 4 k T \Gamma_{\mathrm{n}} \cdot 4 g_{\mathrm{m} 1} \cdot \frac{1}{4}+4 k T \Gamma_{\mathrm{p}} g_{\mathrm{m} 1}=4 k T\left(\Gamma_{\mathrm{n}}+\Gamma_{\mathrm{p}}\right) g_{\mathrm{m} 1}
\end{aligned}
$$

where $\Gamma_{\mathrm{n}}$ and $\Gamma_{\mathrm{p}}$ are approximately $2 / 3$ for long-channel $(L \geq 1.0 \mu \mathrm{m})$ devices, but could be $1-2$ in short-channel devices. Since $G_{\mathrm{M} 1} \approx 0.5 g_{\mathrm{m} 1}$, the noise factor coefficient, $K_{\mathrm{NF}, 1}$, is calculated per Eq. (2.42)

$$
K_{\mathrm{NF}, 1}=\frac{4 k T\left(\Gamma_{\mathrm{n}}+\Gamma_{\mathrm{p}}\right) g_{\mathrm{m} 1}}{4 k T 0.5 g_{\mathrm{m} 1}}=2\left(\Gamma_{\mathrm{n}}+\Gamma_{\mathrm{p}}\right)
$$

Similarly, for $G_{\mathrm{M} 2}\left(M_{3}\right.$ in Fig. 2-29(a)), using the conclusion from Eq. (2.67), we have

$$
i_{\mathrm{no}, \mathrm{gm} 2}^{2}=i_{\mathrm{n}, \mathrm{M} 3}^{2}+i_{\mathrm{n}, \mathrm{MIF}}^{2}=4 k T \Gamma_{\mathrm{n}} g_{\mathrm{m} 3}+4 k T \Gamma_{\mathrm{p}} g_{\mathrm{mIF}} \approx 4 k T\left(\Gamma_{\mathrm{n}}+\Gamma_{\mathrm{p}}\right) g_{\mathrm{m} 3}
$$


Since $G_{\mathrm{M} 2}=-g_{\mathrm{m} 3}$, we have

$$
K_{\mathrm{NF}, 2}=\frac{4 k T\left(\Gamma_{\mathrm{n}}+\Gamma_{\mathrm{p}}\right) g_{\mathrm{m} 3}}{4 k T g_{\mathrm{m} 3}}=\Gamma_{\mathrm{n}}+\Gamma_{\mathrm{p}}
$$
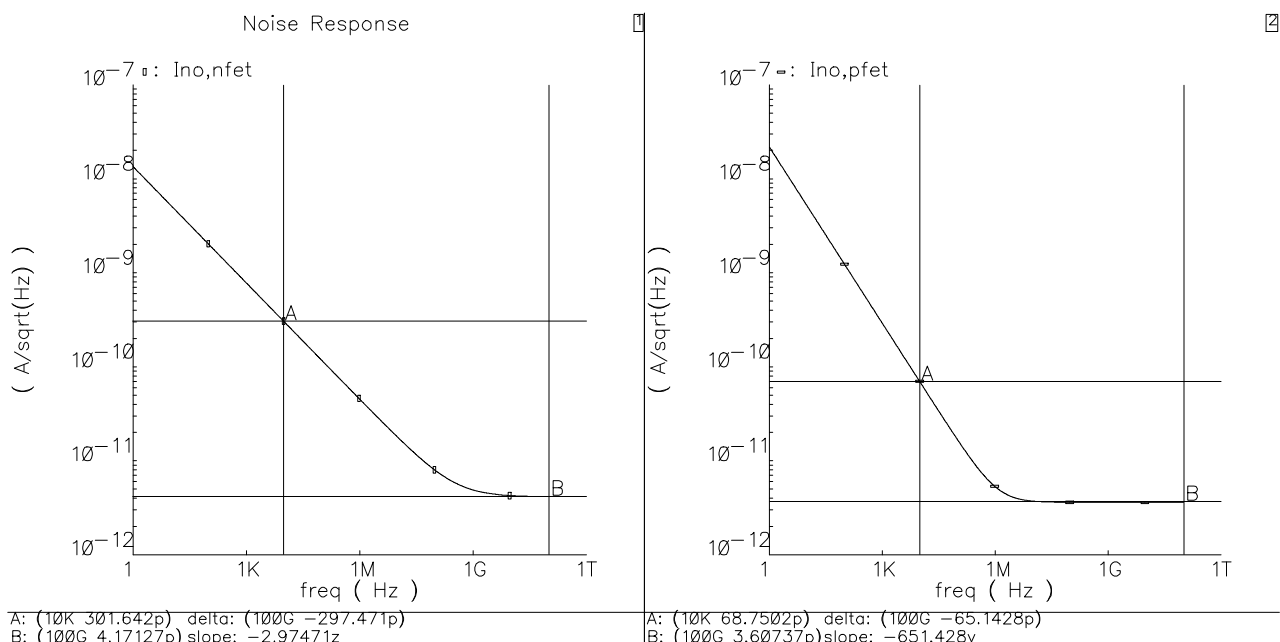

Figure 2-32 The output noise currents of an NFET and a PFET in TSMC 0.18- $\mu \mathrm{m}$ CMOS. Cursor A rests on $1 / f$ noise region; cursor $\mathrm{B}$ rests on thermal noise region.

To use the above results, we need to know the noise parameters of the MOSFETs used in the all-NMOS active inductor. The TSMC device BSIM3 models have noise parameters available, of course; however, the equations for calculating noise using them are very complex, and we need something simpler for hand calculation. Therefore, we will extract simple noise model parameters from simulations. Figure 2-32 shows the output noise current of a 3.0/0.2 $\mu \mathrm{m}$ NFET and 6.0/0.2 $\mu \mathrm{m}$ PFET, which are used in the active-inductor circuit (varactor noise is not considered). Using the following MOSFET noise model [46],

$$
\frac{i_{\mathrm{dn}}^{2}}{\Delta f}=\frac{K_{\mathrm{g}}}{W \cdot L \cdot f^{t}} g_{\mathrm{m}}^{2}+4 k T \Gamma g_{\mathrm{m}}
$$

the noise model parameters can be extracted from simulation results: 


$$
\begin{aligned}
& i_{\mathrm{dn}, \mathrm{nfet}}^{2}=\frac{7.622 \times 10^{-23}}{W \cdot L \cdot f^{0.825}} g_{\mathrm{m}, \mathrm{n}}^{2}+4 k T \cdot 0.8786 \cdot g_{\mathrm{m}, \mathrm{n}}, \text { extracted on } 3.0 / 0.2 \mu \text { NFET } \\
& i_{\mathrm{dn}, \mathrm{pfet}}^{2}=\frac{8.599 \times 10^{-22}}{W \cdot L \cdot f^{1.25}} g_{\mathrm{m}, \mathrm{p}}^{2}+4 k T \cdot 0.9685 \cdot g_{\mathrm{m}, \mathrm{p}}, \text { extracted on } 6.0 / 0.2 \mu \text { PFET }
\end{aligned}
$$

The flicker noise corner frequencies are $318 \mathrm{MHz}$ and $1.1 \mathrm{MHz}$ for the NFET and PFET at the above W/Ls, respectively. Ignoring the effect of flicker noise, the noise factor coefficients for $G_{\mathrm{M} 1}$ and $G_{\mathrm{M} 2}$ are calculated to be $K_{\mathrm{NF} 1}=3.6942$ and $K_{\mathrm{NF} 2}$ $=1.8471$, respectively. Plugging in $G_{\mathrm{M} 1} \approx g_{\mathrm{m} 2}=1.1 \mathrm{mS}, G_{\mathrm{M} 2} \approx g_{\mathrm{m} 3}=1.2 \mathrm{mS}$, we expect their output noise currents being

$$
\begin{aligned}
& i_{\mathrm{no}, \mathrm{gm} 1}=\sqrt{4 k T G_{\mathrm{M} 1} K_{\mathrm{NF} 1}}=8.20 \mathrm{pA} / \sqrt{\mathrm{Hz}} \\
& i_{\mathrm{n}, \mathrm{gm} 2}=\sqrt{4 k T G_{\mathrm{M} 2} K_{\mathrm{NF} 1}}=6.06 \mathrm{pA} / \sqrt{\mathrm{Hz}}
\end{aligned}
$$

which are close to the simulation results of $6.47 \mathrm{pA} / \mathrm{Hz}^{1 / 2}$ and $5.89 \mathrm{pA} / \mathrm{Hz}^{1 / 2}$ shown in Fig. 2-33. The difference for $i_{\text {no,gm1 }}$ is mainly because the lowpass filtering effect of $C_{2}$ at node 2 is ignored in the above analysis. $C_{2}$ will reduce the noise appearing at the output of $G_{\mathrm{M} 1}$.

The noise of the active inductor in Fig. 2-29(a) built in a pseudo-differential structure was simulated and extracted for half of the circuit, as shown in Fig. 2-32. $I_{\mathrm{no}, \mathrm{gm} 1}$ and $i_{\mathrm{no,gm} 2}$ are the equivalent output noise current of $G_{\mathrm{M} 1}\left(M_{1}\right.$ and $M_{2}$ in Fig. 229(a)) and $G_{\mathrm{M} 2}\left(M_{3}\right.$ in Fig. 2-29(a)), respectively, and can be approximately obtained by ac grounding the input node and placing a current probe with correct de voltage at the output node. Also shown is the single-ended output noise current $i_{\mathrm{n}, \mathrm{o} 1}$ (panel 2). 


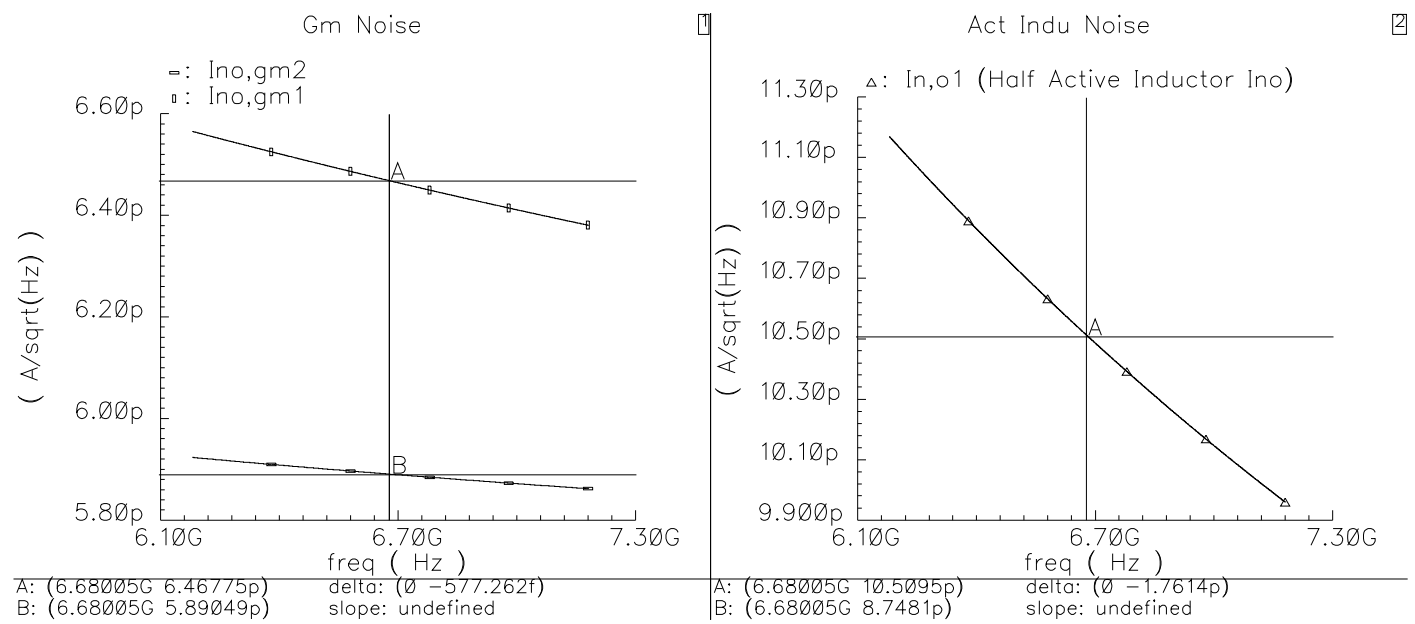

Figure 2-33 Noise simulation results of the $G_{\mathrm{M}}$ cells in the active inductor in Fig. 2-29(a). The biasing conditions are given in Fig. 2-29. Panel 1 shows the output noise current of $G_{\mathrm{M} 1}$ and $G_{\mathrm{M} 2}$, respectively. Panel 2 shows the output noise current of the active inductor. All data are extracted from half of the circuit in a pseudo-differential structure.

Using Eq. (2.50) in Section 2.6, the equivalent shunt noise current of the active inductor at $f_{\mathrm{R}}$ is expected to be

$$
i_{\mathrm{n}}=\sqrt{4 k T\left(K_{\mathrm{NF} 2} G_{\mathrm{M} 2}+\frac{\omega_{\mathrm{R}}^{2}}{\omega_{\mathrm{R}}^{2}} K_{\mathrm{NF} 1} G_{\mathrm{M} 1}\right)}=10.2 \mathrm{pA} / \sqrt{\mathrm{Hz}}
$$

very close to the simulation results of $10.51 \mathrm{pA} / \mathrm{Hz}^{1 / 2}$ shown in Fig. 2-33 (Panel 2).

Per Eq. (2.51), the noise voltage across the active inductor port is the equivalent shunt noise current times the port impedance. This is confirmed by the noise simulation results of the prototype active inductor shown in Fig. 2-34. $i_{\mathrm{n}, \mathrm{o}}\left(\mathrm{A} / \mathrm{Hz}^{1 / 2}\right)$ is the equivalent shunt noise current measured across the two terminals, $V_{\mathrm{oa}}$ and $V_{\mathrm{ob}}$, using a current probe. $\operatorname{Mag}\left(Z_{\text {in }}\right)$ is the magnitude of $Z_{\text {in }}$ measured across terminals $V_{\text {oa }}$ and $V_{\mathrm{ob}}$. It is found that the equivalent output noise voltage, $v_{\mathrm{n}, \mathrm{o}}\left(\mathrm{V} / \mathrm{Hz}{ }^{1 / 2}\right)$, completely overlays the product of $\operatorname{mag}\left(Z_{\text {in }}\right)$ and $i_{\mathrm{n}, \mathrm{o}}$, as predicted by Eq. (2.51). 


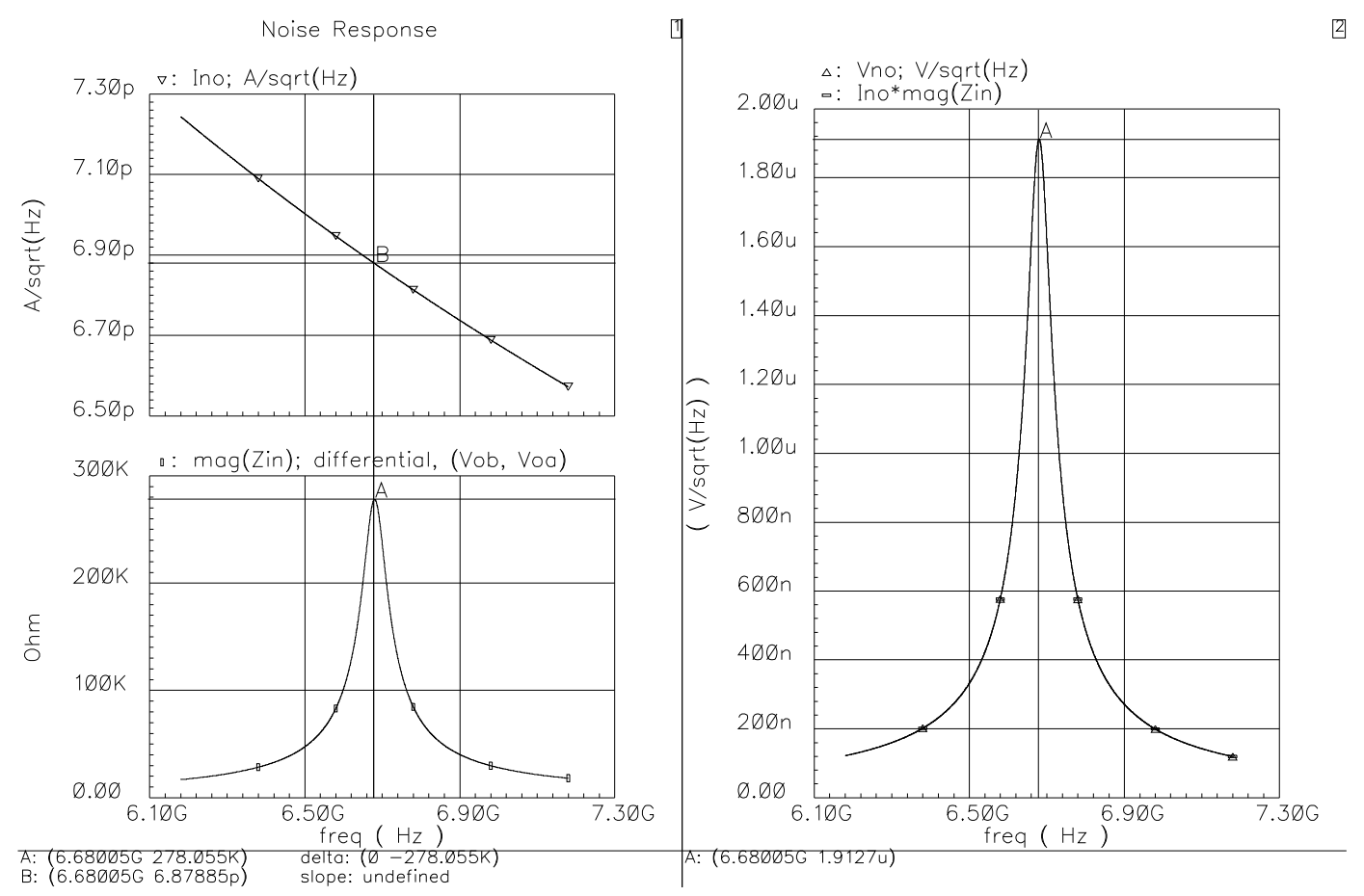

Figure 2-34 The noise of the active inductor shown in Fig. 2-29.

Table 2-2 All-NMOS active inductor noise summary

\begin{tabular}{|l|l|r|r|l|}
\hline \hline Parameter & Unit & \multicolumn{1}{|c|}{ Sims } & \multicolumn{1}{c|}{ Calc'ed } & Equation \\
\hline GammaN & & 0.8786 & & $(2.73)$ \\
\hline GammaP & & 0.9685 & & $(2.73)$ \\
\hline KNF1 & & 3.6942 & & $(2.69)$ \\
\hline KNF2 & & 1.8471 & & $(2.71)$ \\
\hline$G_{\mathrm{M} 1}$ & $\mathrm{mS}$ & 1.14 & & $\mathrm{Tab}(2.1)$ \\
\hline$G_{\mathrm{M} 2}$ & $\mathrm{mS}$ & 1.15 & & $\mathrm{Tab}(2.1)$ \\
\hline$i_{\mathrm{n}, \mathrm{gm} 1}$ & $\mathrm{pA} / \mathrm{sqrt}(\mathrm{Hz})$ & 6.47 & 7.82 & $(2.74)$ \\
\hline$i_{\mathrm{n}, \mathrm{gm} 2}$ & $\mathrm{pA} / \mathrm{sqrt}(\mathrm{Hz})$ & 5.89 & 5.53 & $(2.74)$ \\
\hline$i_{\mathrm{n}, 01}$ & $\mathrm{pA} / \mathrm{sqrt}(\mathrm{Hz})$ & 10.51 & 10.24 & $(2.75)$ \\
\hline$i_{\mathrm{n}, \mathrm{o}}$ & $\mathrm{pA} / \mathrm{sqrt}(\mathrm{Hz})$ & 6.88 & 7.24 & $(2.65)$ \\
\hline$Z_{\mathrm{in}}(\mathrm{between}$ Voa and & & & & \\
Vob) & $\mathrm{kOhm}$ & 278.0 & 273.3 & $(2.62)$ \\
\hline$v_{\mathrm{n}, \mathrm{o}}$ & $\mathrm{uV} / \mathrm{sqrt}(\mathrm{Hz})$ & 1.91 & 1.98 & $(2.51)$ \\
\hline All at $f_{\mathrm{R}}=6.68 \mathrm{GHz}$ & & & & \\
\hline
\end{tabular}

Table 2-2 summarizes the all-NMOS active-inductor noise simulation and calculated results. The $Z_{\text {in }}$ is about twice that of the value in Table $2-1$, because it is taken differentially here between $V_{\mathrm{oa}}$ and $V_{\mathrm{ob}}$. The calculated results match the 
simulation results.

\subsection{All-NMOS-II Active-Inductor Circuit (New in This Work)}

This circuit was initially developed as a precision analog signal driver, with a constant unity gain and very high linearity. But when configured differently, it can be an active inductor with an all-NMOS signal path and hence was designated "all-NMOS-II active inductor". It has the same high-frequency capability of the previous circuit with a 1.414 times improvement in $f_{\mathrm{R}}$.

\subsubsection{The Circuit as a Precision Analog Signal Driver}

The primary circuit is shown in Fig. 2-35. $M_{\mathrm{B} 1-3}$ are biasing transistors acting as current sources. $M_{\mathrm{B} 1}$ and $M_{\mathrm{B} 2}$ are matched, and $M_{\mathrm{B} 3}$ can be different depending on the application requirement. For reasons we will see below, $M_{\mathrm{B} 1}$ and $M_{\mathrm{B} 2}$ have almost identical $V_{\mathrm{ds}} \mathrm{s}$ with low dynamic variation, hence cascode is not necessary to ensure their $I_{\mathrm{DS}}$ match. And we will find that the $g_{\mathrm{ds}}$ of $M_{\mathrm{B} 3}$ does not affect the circuit's performance and hence cascode is not necessary on $M_{\mathrm{B} 3}$ either. $M_{1}$ and $M_{2}$ have identical aspect ratios and their $W$ and $L$ are chosen based on the requirements on the maximum operating frequency and $V_{\mathrm{IN}}-V_{\mathrm{OUT}} \mathrm{dc}$ mismatch.

$M_{3}$ and $M_{4}$ have identical length, which is chosen based on frequency requirement and process variation, and their widths are chosen according to

$$
\frac{W_{\mathrm{M} 4}}{W_{\mathrm{M} 3}}=\frac{I_{1}+I_{2}}{I_{3}}=\frac{2 I_{1}}{I_{3}}
$$




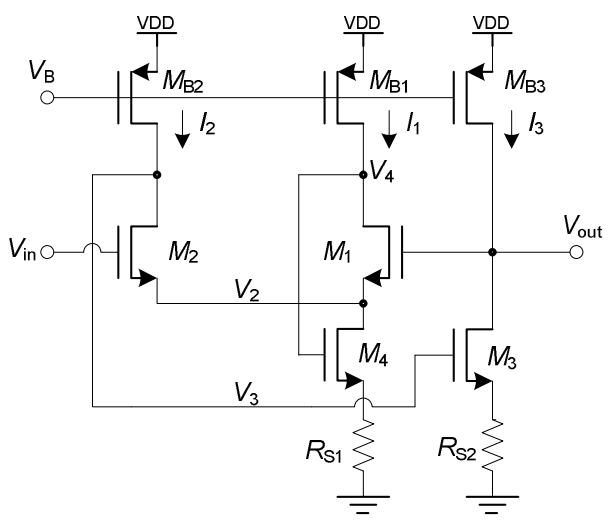

Figure 2-35 The all-NMOS-II circuit configured as a precision analog signal driver.

The source degeneration resistors $R_{\mathrm{S} 1}$ and $R_{\mathrm{S} 2}$ are optional, and merely create an extra degree of freedom in the circuit design. $R_{\mathrm{S} 1}$ raises the source voltage of $M_{4}$ by a fixed amount, and $R_{\mathrm{S} 2}$ degenerates $M_{3}$ and reduces the capacitance looking into the $M_{3}$ gate. Their values are chosen to satisfy

$$
R_{\mathrm{S} 1}\left(I_{1}+I_{2}\right)=R_{\mathrm{S} 2} I_{3} \quad \text { or } \quad V_{\mathrm{S} 4}=V_{\mathrm{S} 3}
$$

Therefore,

$$
V_{3}=V_{\mathrm{gs} 3}+V_{\mathrm{S} 3}=V_{\mathrm{gs} 4}+V_{\mathrm{S} 4}=V_{4}
$$

Since $M_{1}$ and $M_{2}$ 's sources are connected to the same node $V_{2}$, we have two desired outcomes at the same time:

$$
V_{\mathrm{ds} 1}=V_{\mathrm{ds} 2} \quad \text { and } \quad V_{\mathrm{ds}, \mathrm{MB} 1}=V_{\mathrm{ds}, \mathrm{MB} 2}
$$

The latter fulfills our identical $V_{\mathrm{ds}}$ assumption on $M_{\mathrm{B} 1}$ and $M_{\mathrm{B} 2}$ above and makes $I_{1}=$ $I_{2}$, since $M_{\mathrm{B} 1}$ and $M_{\mathrm{B} 2}$ have the same geometry and $V_{\mathrm{GS}}$. The former, together with

$$
I_{\mathrm{d}, \mathrm{M} 1}=I_{\mathrm{d}, \mathrm{M} 2}, \quad V_{\mathrm{t}, \mathrm{M} 1}=V_{\mathrm{t}, \mathrm{M} 2}, \quad W_{\mathrm{M} 1}=W_{\mathrm{M} 2}, L_{\mathrm{M} 1}=L_{\mathrm{M} 2}
$$

yields

$$
V_{\mathrm{gs}, \mathrm{M} 1}=V_{\mathrm{gs}, \mathrm{M} 2} \text {, or } V_{\mathrm{gs} 1}=V_{\mathrm{gs} 2}
$$


after simplifying the notation. This is guaranteed by the feedbacks within the circuit, of course, since $V_{\mathrm{gs}}$ controls $I_{\mathrm{d}}$ and $I_{\mathrm{d}}$ does not control $V_{\mathrm{gs}}$. Since

$$
V_{\text {in }}-V_{2}=V_{\mathrm{gs} 2}, V_{\text {out }}-V_{2}=V_{\mathrm{gs} 1}
$$

we have

$$
V_{\text {in }}=V_{\text {out }}
$$

and this is enforced by the feedback through $M_{3}$.

Assuming that when the circuit is in dynamic operation, $V_{\text {in }}$ increases by $\Delta V_{\text {in }}(>$ 0 ), and consequently $V_{\text {out }}$ and $V_{2}$ both increase by (approximately) $\Delta V_{\text {in. }}$. Using the MOSFET I-V equation, when their $I_{\mathrm{DS}}$ 's are constant ${ }^{4}$, the $V_{\mathrm{gs}}$ change of $M_{3}$ and $M_{4}$ from their $V_{\mathrm{ds}}$ change through the channel-length modulation effect is solved as

$$
\frac{d V_{\mathrm{gs}}}{d V_{\mathrm{ds}}}=\frac{d\left(V_{\mathrm{T}}+V_{\mathrm{od}}\right)}{d V_{\mathrm{ds}}}=-\frac{\lambda V_{\mathrm{od}}}{2\left(1+\lambda V_{\mathrm{ds}}\right)} \approx-\frac{\lambda V_{\mathrm{od}}}{2}=-\frac{g_{\mathrm{ds}} V_{\mathrm{od}}}{2 I_{\mathrm{ds}}}
$$

Note that $\lambda$ is a very small number, and hence $d V_{\mathrm{gs}} / d V_{\mathrm{ds}}$ is very small. Since $M_{3}$ and $M_{4}$ have identical $V_{\text {od }}$ and $\lambda$, they have the identical $V_{\mathrm{gs}}$ change of

$$
\Delta V_{\mathrm{gs} 3,4}=\Delta V_{3}=\Delta V_{4} \approx-\frac{\lambda V_{\mathrm{od} 3}}{2} \Delta V_{\mathrm{in}}
$$

again, a very small number. The $V_{\mathrm{ds}}$ changes for $M_{1}$ and $M_{2}$ will be

$$
\begin{aligned}
& \Delta V_{\mathrm{ds} 1}=\Delta V_{4}-\Delta V_{2} \approx-\frac{\lambda V_{\mathrm{od} 4}}{2} \Delta V_{\mathrm{in}}-\Delta V_{\mathrm{in}}=-\Delta V_{\mathrm{in}}\left(1+\frac{\lambda V_{\mathrm{od} 4}}{2}\right) \\
& \Delta V_{\mathrm{ds} 2}=\Delta V_{3}-\Delta V_{2} \approx-\frac{\lambda V_{\mathrm{od} 3}}{2} \Delta V_{\mathrm{in}}-\Delta V_{\mathrm{in}}=-\Delta V_{\text {in }}\left(1+\frac{\lambda V_{\mathrm{od} 3}}{2}\right)=\Delta V_{\mathrm{ds} 1}
\end{aligned}
$$

\footnotetext{
${ }^{4}$ The $I_{\mathrm{DS}}$ 's of $M_{3}$ and $M_{4}$ will change slightly due to the channel-length modulation effect on $M_{\mathrm{B} 1-3}$ that is determined by the solution of the next few equations. This has very little effect and is neglected to simplify the discussion.
} 
$\Delta V_{3}$ and $\Delta V_{4}$, both $<0$, will increase $I_{1}$ and $I_{2}$ slightly by the same amount through the channel-length modulation of $M_{\mathrm{B} 1}$ and $M_{\mathrm{B} 2}$, and $\Delta V_{\mathrm{ds} 1}$ and $\Delta V_{\mathrm{ds} 2}$, both $<0$, will increase $V_{\mathrm{gs} 1}$ and $V_{\mathrm{gs} 2}$ slightly by the same amount. Per Eq. (2.82),

$$
\begin{aligned}
& \Delta\left(V_{\text {out }}-V_{\text {in }}\right)=\Delta\left[\left(V_{2}+V_{\mathrm{gs} 1}\right)-\left(V_{2}+V_{\mathrm{gs} 2}\right)\right]=\Delta\left(V_{\mathrm{gs} 1}-V_{\mathrm{gs} 2}\right) \\
& =\Delta\left(\Delta V_{\mathrm{gs} 1}-\Delta V_{\mathrm{gs} 2}\right)=0
\end{aligned}
$$

Hence, the $2^{\text {nd }}$-order effects of MOSFETs have no direct effect on $\Delta\left(V_{\text {out }}-V_{\text {in }}\right)$. The $2^{\text {nd }}$-order effects are repeatedly subtracted from each other before they reach $\Delta\left(V_{\text {out }}-\right.$ $V_{\text {in), }}$, that is

$$
A_{\mathrm{V}}=\frac{d V_{\text {out }}}{d V_{\text {in }}}=\frac{d}{d V_{\text {in }}}\left(V_{\mathrm{gs} 1}-V_{\mathrm{gs} 2}+V_{\text {in }}\right)=\frac{d}{d V_{\text {in }}}\left(V_{\mathrm{gs} 1}-V_{\mathrm{gs} 2}\right)+1=1
$$

Therefore, we have $V_{\text {out }}=V_{\text {in }}$ and $\Delta V_{\text {out }}=\Delta V_{\text {in, }}$ and three interesting results ensue:

1. The dc (or common-mode) voltages $V_{\text {OUT }}$ and $V_{\text {IN }}$ are the same, less the $V_{\mathrm{T}}$ mismatch between $M_{1}$ and $M_{2}$.

2. $\Delta V_{\text {out }}=\Delta V_{\text {in }}$ and the gain is equal to 1.0. $V_{\mathrm{T}}$ mismatches have no direct impact on the gain (Eq. 2.85) and consequently the gain is constant across processtemperature corners.

3. Since $V_{\text {out }}$ follows $V_{\text {in }}$ exactly, the small-signal linearity (IIP3) is high and the linearity is preserved over a wide input signal range.

\subsubsection{Analysis using Feedback Theory}

The constant gain and high linearity of the circuit may also be explained using the 
feedback theory. Considering small signals only, the drain current of $M_{1}$ is $g_{\mathrm{m} 1}\left(V_{\text {out }}-\right.$ $V_{2}$ ), and it is converted into voltage $V_{4}$ by multiplying the total conductance looking into node $V_{4}, g_{4} . V_{4}$ is further transferred to $V_{2}$ by $g_{\mathrm{m} 4} /\left(g_{\mathrm{m} 1}+g_{\mathrm{m} 2}\right)$, as shown by the block diagram in Fig. 2-36:

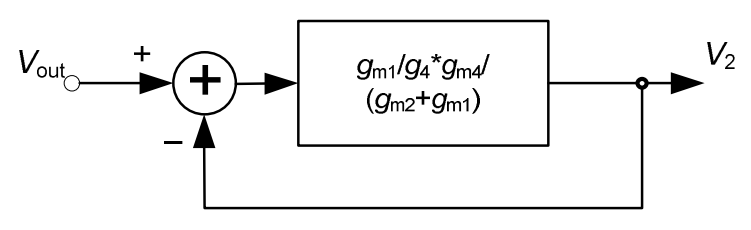

Figure 2-36 The local feedback loop within the circuit.

The transfer function is

$$
\frac{V_{2}}{V_{\text {out }}}=\left.\frac{\frac{g_{\mathrm{ml}}}{g_{4}} \frac{g_{\mathrm{m} 4}}{g_{\mathrm{m}}+g_{\mathrm{m} 2}}}{1+\frac{g_{\mathrm{ml}}}{g_{4}} \frac{g_{\mathrm{m} 4}}{g_{\mathrm{ml}}+g_{\mathrm{m} 2}}}\right|_{V_{\text {od1 }}=V_{\text {od } 2} \approx V_{\text {od }} \text {, and Eq(2.67) }} \approx \frac{\frac{g_{\mathrm{ml}}}{g_{4}} \frac{2 g_{\mathrm{ml}}}{g_{\mathrm{ml}}+g_{\mathrm{ml}}}}{1+\frac{g_{\mathrm{ml}}}{g_{4}} \frac{2 g_{\mathrm{m}}}{g_{\mathrm{ml}}+g_{\mathrm{ml}}}}=\left.\frac{\frac{g_{\mathrm{ml}}}{g_{4}} \cdot 1}{1+\frac{g_{\mathrm{ml}}}{g_{4}} \cdot 1}\right|_{g_{\mathrm{ml}} / g_{4}>>1} \approx 1
$$

Next $V_{\text {in }}$ and $V_{2}$ are subtracted by $M_{2}$, whose drain current is converted into the voltage $V_{3}$ by the total conductance looking into node $V_{3}, g_{3}$, and $V_{3}$ is transferred to $V_{\text {out }}$ by $g_{\mathrm{m} 3}^{\prime} / g_{\text {out }}\left(g_{\mathrm{m} 3}^{\prime}=g_{\mathrm{m} 3} /\left(1+g_{\mathrm{m} 3} R_{\mathrm{S} 2}\right)\right.$ is $g_{\mathrm{m} 3}$ with the degeneration effect of $\left.R_{\mathrm{S} 2}\right) \cdot g_{\text {out }}$ is the total conductance looking into node $V_{\text {out }}$. This process can be represented by the diagram in Fig. 2-37:

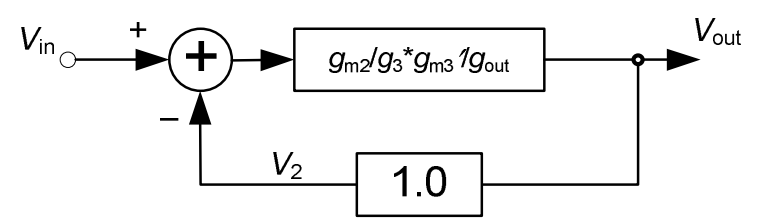

Figure 2-37 The main feedback loop within the circuit.

Its transfer function is

$$
A_{\mathrm{V}}=\left.\frac{g_{\mathrm{m} 2} g_{\mathrm{m} 3}^{\prime} /\left(g_{3} g_{\text {out }}\right)}{1+1 \cdot g_{\mathrm{m} 2} g_{\mathrm{m} 3}^{\prime} /\left(g_{3} g_{\text {out }}\right)}\right|_{\frac{g_{\mathrm{m}} g_{3}^{\prime} 3}{g_{3} g_{\text {out }}>1}} \approx 1
$$


The loop gain of the main feedback is $\propto\left(g_{\mathrm{m}} / g_{\mathrm{ds}}\right)^{2}$, that is, very high; the local feedback loop gain is $\propto\left(g_{\mathrm{m}} / g_{\mathrm{ds}}\right)$ and lower, but sufficient. The voltage gain of the circuit is 1 because the feedback factors of the two loops are both 1, determined by the circuit architecture, not by component matching, and the high loop gain ensures that the voltage gain is constant with process, temperature variations, and device mismatches.

\subsubsection{AC Small-Signal Analysis}

For this circuit, we may of course draw the entire ac equivalent circuit and conduct a symbolic circuit analysis. However, due to the high number of parasitic capacitors, the transfer function will be high-order and the mathematics will be too complex to bring any design insights. Therefore, we will break the circuit into functional blocks with unilateral signal transfer functions, and judiciously neglect parasitic terms in the circuit equations.

The circuit is decomposed into three blocks: voltage transfer block from $V_{\text {out }}$ to $V_{2} ; M_{2}$ as a noninverting transconductor with input being $V_{2}$ and output at $V_{3} ; M_{3}$ as a source-degenerated inverting transconductor with input being $V_{3}$ and output at $V_{\text {out. }}$ The ac small-signal equivalent circuit of the voltage transfer block is shown in Fig. 238, where $1 / g_{\mathrm{m} 2}$ is the resistance looking into the $M_{2}$ source and is the load of the block, $g_{4}$ and $C_{4}$ are the parasitic conductance and capacitance at $V_{4}$, respectively. From the node equations for $V_{4}$ and $V_{2}$, we solve the transfer function as ${ }^{5}$

\footnotetext{
${ }^{5}$ The circuit is assumed to be driven by a voltage source with zero source impedance, a reasonable and acceptable assumption for this circuit, since the capacitive loading effect of the $M_{2}$ gate to the $M_{3}$ drain is considered in the following analysis.
} 


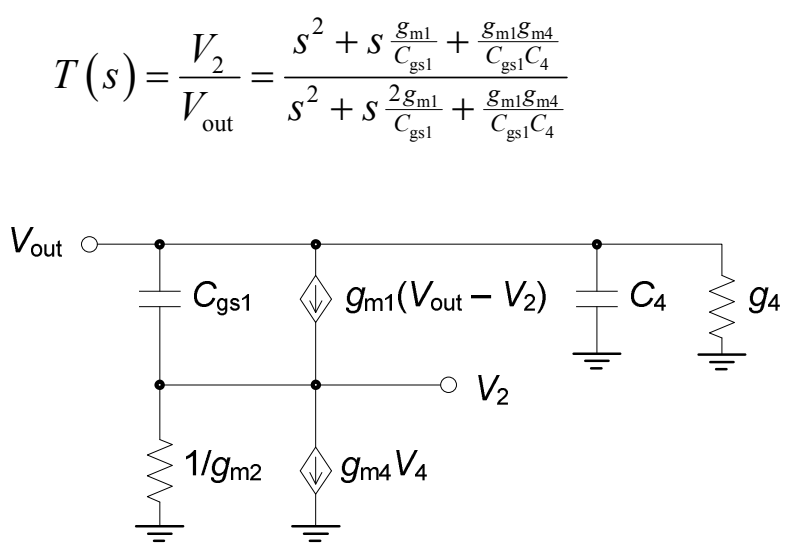

Figure 2-38 Small-signal equivalent circuit from $V_{\text {out }}$ to $V_{2}$.

Its pole frequency and $Q$ s are

$$
\omega_{\mathrm{p} 1}=\sqrt{\frac{g_{\mathrm{m} 1} g_{\mathrm{m} 4}}{C_{\mathrm{gs} 1} C_{4}}}, \quad Q_{\mathrm{p} 1}=\frac{1}{2} \sqrt{\frac{g_{\mathrm{m} 4} C_{\mathrm{gs} 1}}{g_{\mathrm{m} 1} C_{4}}}, \quad Q_{\mathrm{z} 1}=\sqrt{\frac{g_{\mathrm{m} 4} C_{\mathrm{gs} 1}}{g_{\mathrm{m} 1} C_{4}}}=2 Q_{\mathrm{p} 1}
$$

$\omega_{\mathrm{p} 1}$ is the ratio of transistor $g_{\mathrm{m}}$ to parasitic capacitor, and as we shall see later, is much larger than the pole frequency of the whole circuit, which contains the large load capacitor at $V_{\text {out }} Q_{\mathrm{p} 1}$ and $Q_{\mathrm{z} 1}$ are both fairly low for practical circuits $\left(Q_{\mathrm{p} 1} \approx 1\right.$ in most cases). Therefore, within the frequency range of interest, it operates almost like a unity-gain filter, that is

$$
T(s)=\frac{V_{2}}{V_{\text {out }}}=\frac{s^{2}+s \frac{g_{\mathrm{m} 1}}{C_{\mathrm{gs} 1}}+\frac{g_{\mathrm{m}} g_{\mathrm{m} 4}}{C_{\mathrm{gs} 1} C_{4}}}{s^{2}+s \frac{2 g_{\mathrm{m} 1}}{C_{\mathrm{gs} 1}}+\frac{g_{\mathrm{m} 1} g_{\mathrm{m} 4}}{C_{\mathrm{gs} 1} C_{4}}} \approx 1, \text { or } V_{2}=V_{\text {out }}
$$

The $Q_{\mathrm{p} 1}$ and $Q_{\mathrm{z} 1}$ difference of a factor of 2 will distort the frequency profile a little bit, but will have little effect since $\omega_{\mathrm{p} 1}$ is much higher than the circuit's operating frequency and $Q_{\mathrm{p} 1}$ and $Q_{\mathrm{z} 1}$ are both low. 


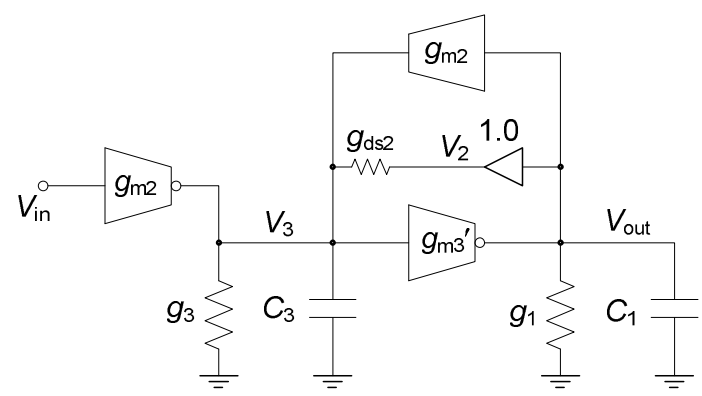

Figure 2-39 Small-signal equivalent circuit of the entire circuit.

Using this result, the small-signal equivalent of the entire circuit is drawn in Fig. 2-39. $g_{\mathrm{m} 2}$ is the $g_{\mathrm{m}}$ of $M_{2}$ and $g^{\prime}{ }_{\mathrm{m} 3}$ is the $g_{\mathrm{m}}$ of $M_{3}$ degenerated by $R_{\mathrm{S} 2}$ :

$$
g_{\mathrm{m} 3}{ }^{\prime}=\frac{g_{\mathrm{m} 3}}{1+g_{\mathrm{m} 3} R_{\mathrm{S} 2}}
$$

The unity-gain buffer on $g_{\mathrm{ds} 2}$ in the figure is from the unilateral isolation effect of $M_{1}$ and $M_{4}$ for $V_{\text {out, }}$, that is, $V_{2}\left(\approx V_{\text {out }}\right)$ can generate current into $V_{3}$ through $g_{\mathrm{ds} 2}$, but the current generated by $V_{3}$ through $g_{\mathrm{ds} 2}$ into $V_{2}$ is totally sunk without affecting $V_{2}$ since $V_{2}$ is low-impedance.

Since the impedance looking into the $M_{3}$ gate is very high and $g_{\mathrm{ds} 2}$ has been taken into account separately, we have

$$
g_{3} \approx g_{\mathrm{ds}, \mathrm{MB} 2}
$$

Using a simple small-signal analysis, the capacitance looking into $M_{3}$ gate with source-degeneration resistor $R_{\mathrm{S} 2}$ is

$$
C_{\mathrm{gs} 3}^{\prime}=\frac{C_{\mathrm{gs} 3}}{1+R_{\mathrm{S} 2}\left(s C_{\mathrm{gs} 3}+g_{\mathrm{m} 3}\right)} \approx \frac{C_{\mathrm{gs} 3}}{1+R_{\mathrm{s} 2} g_{\mathrm{m} 3}}
$$

because at the circuit's pole frequency, $\left|s C_{\mathrm{gs} 3}\right|<<g_{\mathrm{m} 3}$, typically at about $1 \%$.

$C_{3}$, the total capacitance at node $V_{3}$, is the sum of junction capacitance and $C_{\mathrm{gs} 3}{ }^{\prime}$, 


$$
C_{3}=C_{\mathrm{jd}, \mathrm{MB} 2}+C_{\mathrm{jd} 2}+\frac{C_{\mathrm{gs} 3}}{1+R_{\mathrm{s} 2} g_{\mathrm{m} 3}}
$$

$C_{1}$ and $g_{1}$ are the total capacitance (mostly the load capacitor) and conductance at node $V_{\text {out }}$, and they are

$$
C_{1}=C_{\mathrm{L}}+C_{\mathrm{jd} 3}+C_{\mathrm{jd}, \mathrm{MB} 3}, \quad g_{1}=g_{\mathrm{ds}, \mathrm{MB} 3}+\frac{1}{R_{\mathrm{S} 2}+\left(1+g_{\mathrm{m} 3} R_{\mathrm{S} 2}\right) / g_{\mathrm{ds} 3}}
$$

Using node analysis, the transfer function of the circuit can be solved as

$$
T(s)=\frac{V_{\text {out }}}{V_{\text {in }}}=\frac{\frac{g_{\mathrm{m} 2} g_{\mathrm{m} 3}^{\prime}}{C_{1} C_{3}}}{s^{2}+s\left(\frac{g_{1}}{C_{1}}+\frac{g_{3}+g_{\mathrm{ds}}}{C_{3}}\right)+\frac{\left(g_{\mathrm{m} 2}+g_{\mathrm{d} 2}\right) g_{\mathrm{m}_{3}+g_{1}\left(g_{3}+g_{\mathrm{d} 2}\right)}}{C_{1} C_{3}}}
$$

$T(s)$ is recognized as a lowpass function, with pole frequency, pole $Q$, and dc gain being

$$
\begin{aligned}
& \omega_{\mathrm{p}}=\sqrt{\frac{\left(g_{\mathrm{m} 2}+g_{\mathrm{ds} 2}\right) g_{\mathrm{m} 3}^{\prime}+g_{1}\left(g_{3}+g_{\mathrm{ds} 2}\right)}{C_{1} C_{3}}} \approx \sqrt{\frac{g_{\mathrm{m} 2} g_{\mathrm{m} 3}^{\prime}}{C_{1} C_{3}}}, \quad Q_{\mathrm{p}}=\frac{\omega_{\mathrm{p}}}{\frac{g_{1}}{C_{1}}+\frac{g_{3}+g_{\mathrm{ds} 2}}{C_{3}}}, \\
& H_{0}=\frac{g_{\mathrm{m} 2} g_{\mathrm{m} 3}^{\prime}}{\left(g_{\mathrm{m} 2}+g_{\mathrm{ds} 2}\right) g_{\mathrm{m} 3}^{\prime}+g_{1}\left(g_{3}+g_{\mathrm{ds} 2}\right)} \approx \frac{g_{\mathrm{m} 2} g_{\mathrm{m} 3}^{\prime}}{g_{\mathrm{m} 2} g_{\mathrm{m} 3}^{\prime}}=1
\end{aligned}
$$




\subsubsection{Design Example}

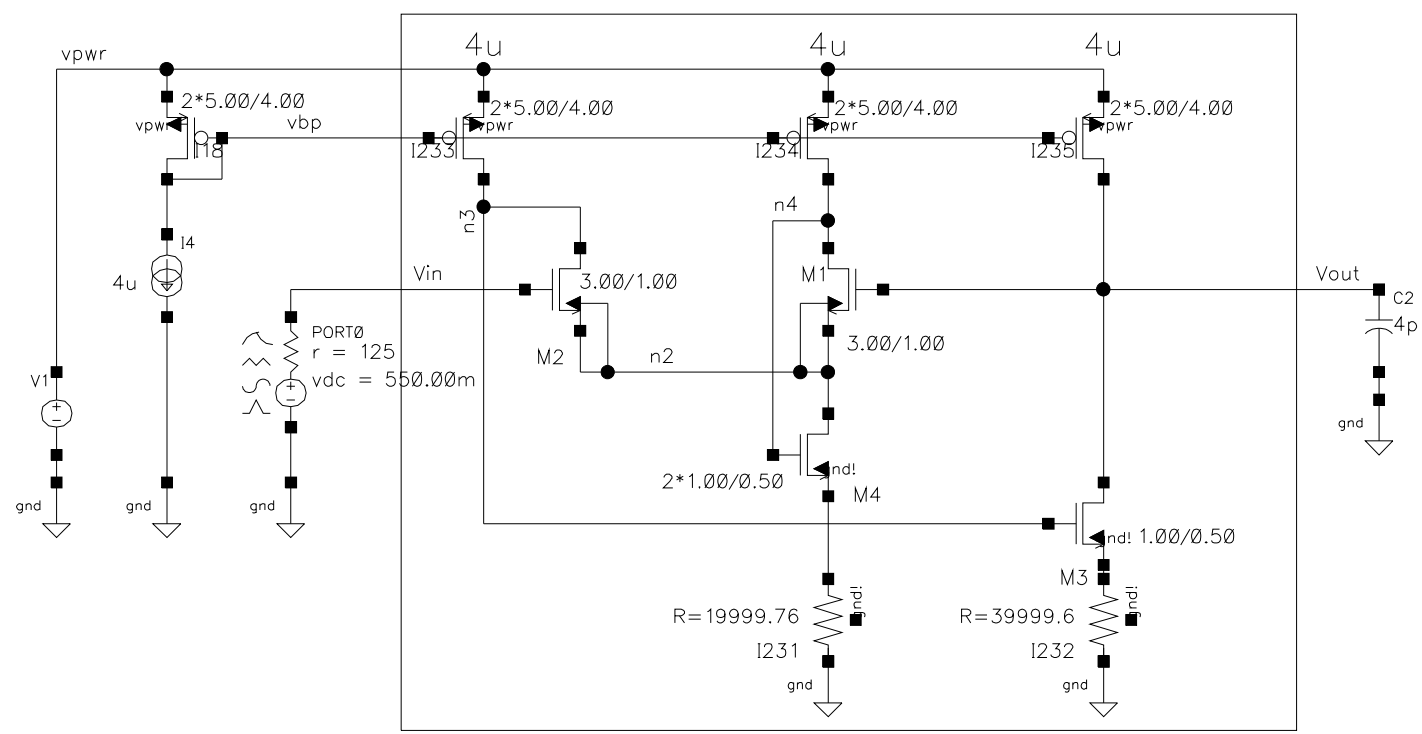

Figure 2-40 Example of All-NMOS-II circuit configured as an analog signal driver.

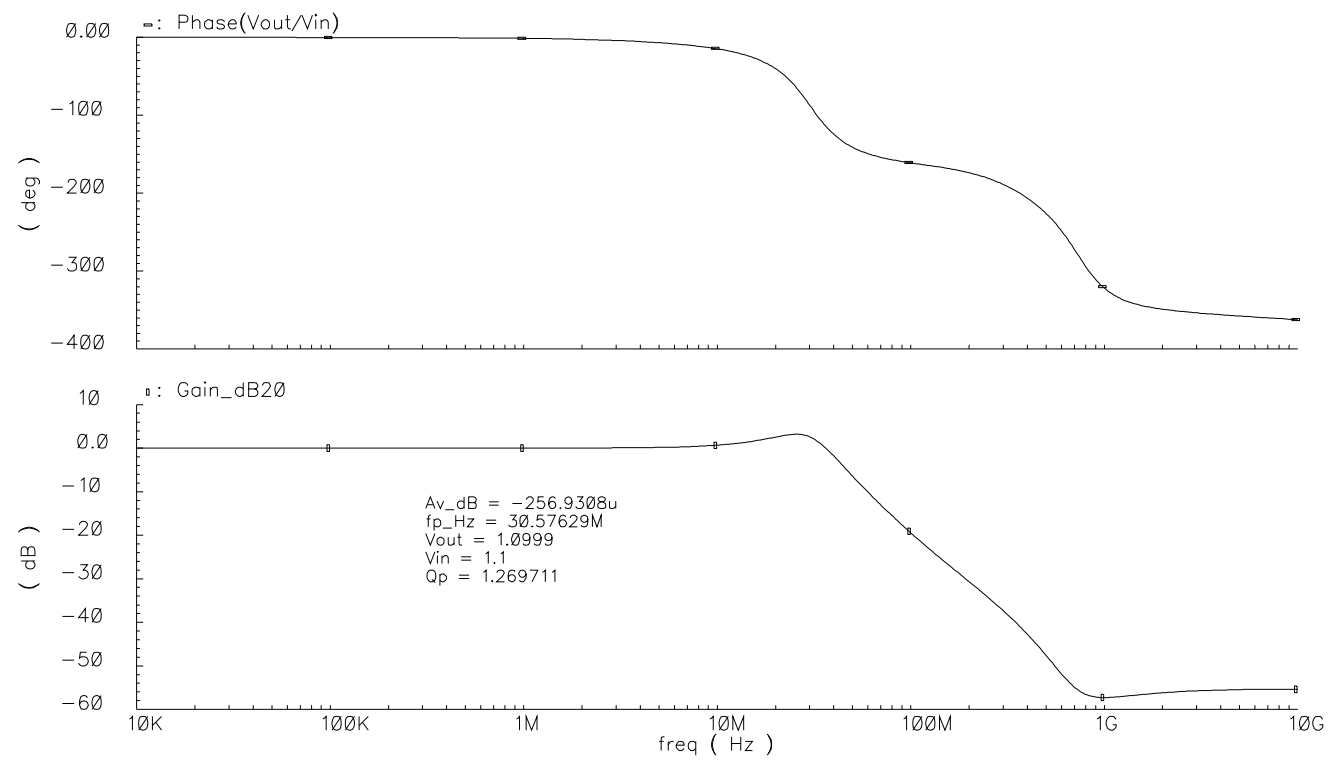

Figure 2-41 The frequency response of the analog signal driver.

Figure 2-40 shows an example of the circuit as an analog signal driver, designed in a submicron CMOS process. As expected, $V_{\mathrm{IN}}$ and $V_{\text {OUT }}$ are identical. The gain is -0.26 $\mathrm{mdB}$, and the pole frequency and $Q$ are $30.6 \mathrm{MHz}$ and 1.27 , respectively, at a $4-\mathrm{pF}$ load. To verify the circuit equations, equivalent-circuit-component parameters are 
obtained from the operating points: $g_{\mathrm{m} 2}=65.32 \mu \mathrm{S}, g_{\mathrm{m} 3}=51.96 \mu \mathrm{S}, C_{1}=4.003 \mathrm{pF}, C_{3}$ $=8 \mathrm{fF}, g_{\mathrm{ds} 2}=1.11 \mu \mathrm{S}, g_{\mathrm{ds}, \mathrm{MB} 2}=80 \mathrm{nS}, g_{\mathrm{ds}, \mathrm{MB} 3}=90 \mathrm{nS}, g_{\mathrm{ds} 3}=0.48 \mu \mathrm{S}, R_{\mathrm{S} 2}=40 \mathrm{k} \Omega$. Using these, we can calculate $g_{1}=0.245 \mu \mathrm{S}, g_{3}=80 \mathrm{nS}, g_{\mathrm{m}{ }^{\prime}}=16.88 \mu \mathrm{S}$. Plugging these into Eq. (2.95), we get $f_{\mathrm{p}}=29.8 \mathrm{MHz}, Q_{\mathrm{p}}=1.26, H_{0}=0.98$, very close to the simulation results. $H_{0}$ is more pessimistic because the cancellation of MOSFET second-order effects discussed in Section 2.8.1 is not included in the small-signal circuit model in Fig. 2-37 for simplicity. The simulation and calculated results are summarized in Table 2-3.

Table 2-3 Calculated versus simulated results for the All-NMOS-II circuit when used an analog signal driver or lowpass filter

\begin{tabular}{|l|l|r|l|l|l|l|r|r|}
\hline Parameter & Unit & Value & Equation & Parameter & Unit & Sims & Calc'ed & Equation \\
\hline$g_{\mathrm{m} 1}$ & $\mathrm{uS}$ & 65.31 & & $f_{\mathrm{p}}$ & $\mathrm{MHz}$ & $\mathbf{3 0 . 5 8}$ & $\mathbf{2 9 . 8}$ & $(2.98)$ \\
\hline$g_{\mathrm{m} 2}$ & $\mathrm{uS}$ & $\mathbf{6 5 . 3 2}$ & & $Q_{\mathrm{p}}$ & & $\mathbf{1 . 2 7}$ & $\mathbf{1 . 2 6}$ & $(2.98)$ \\
\hline$g_{\mathrm{m} 3}$ & $\mathrm{uS}$ & $\mathbf{5 1 . 9 6}$ & & $H_{0}$ & & $\mathbf{1 . 0 0}$ & $\mathbf{0 . 9 8}$ & $(2.98)$ \\
\hline$C_{1}$ & $\mathrm{pF}$ & $\mathbf{4 . 0 0 3}$ & $(2.98)$ & & & & & \\
\hline$C_{3}$ & $\mathrm{fF}$ & $\mathbf{8}$ & $(2.97)$ & $V_{\mathrm{DD}}$ & $\mathrm{V}$ & $\mathbf{1 . 8}$ & & \\
\hline & & & \multicolumn{1}{|c|}{$\Delta V_{\mathrm{in}}($ gain } \\
$g_{\mathrm{ds} 2}$ & $\mathrm{uS}$ & $\mathbf{1 . 1 1}$ & & varies by $1 \%)$ & $\mathrm{mV}$ & $\mathbf{5 7 0}$ & & \\
\hline$g_{\mathrm{ds}, \mathrm{MB} 1}$ & $\mathrm{nS}$ & 80 & & IIP3 & $\mathrm{Vp}$ & $\mathbf{3 9 . 5}$ & & \\
\hline$g_{\mathrm{ds}, \mathrm{MB} 2}$ & $\mathrm{nS}$ & 80 & & & & & & \\
\hline$g_{\mathrm{ds}, \mathrm{MB} 3}$ & $\mathrm{nS}$ & 90 & & & & & & \\
\hline$g_{\mathrm{d} 33}$ & $\mathrm{uS}$ & 0.48 & & & & & & \\
\hline$R_{\mathrm{S} 2}$ & $\mathrm{kOhm}$ & 40 & & & & & & \\
\hline$g_{1}$ & $\mathrm{uS}$ & $\mathbf{0 . 2 4 5}$ & $(2.98)$ & & & & & \\
\hline$g_{3}$ & $\mathrm{nS}$ & $\mathbf{8 0}$ & $(2.95)$ & & & & & \\
\hline$g_{\mathrm{m} 3}^{\prime}$ & $\mathrm{uS}$ & $\mathbf{1 6 . 8 8}$ & $(2.94)$ & & & & \\
\hline
\end{tabular}



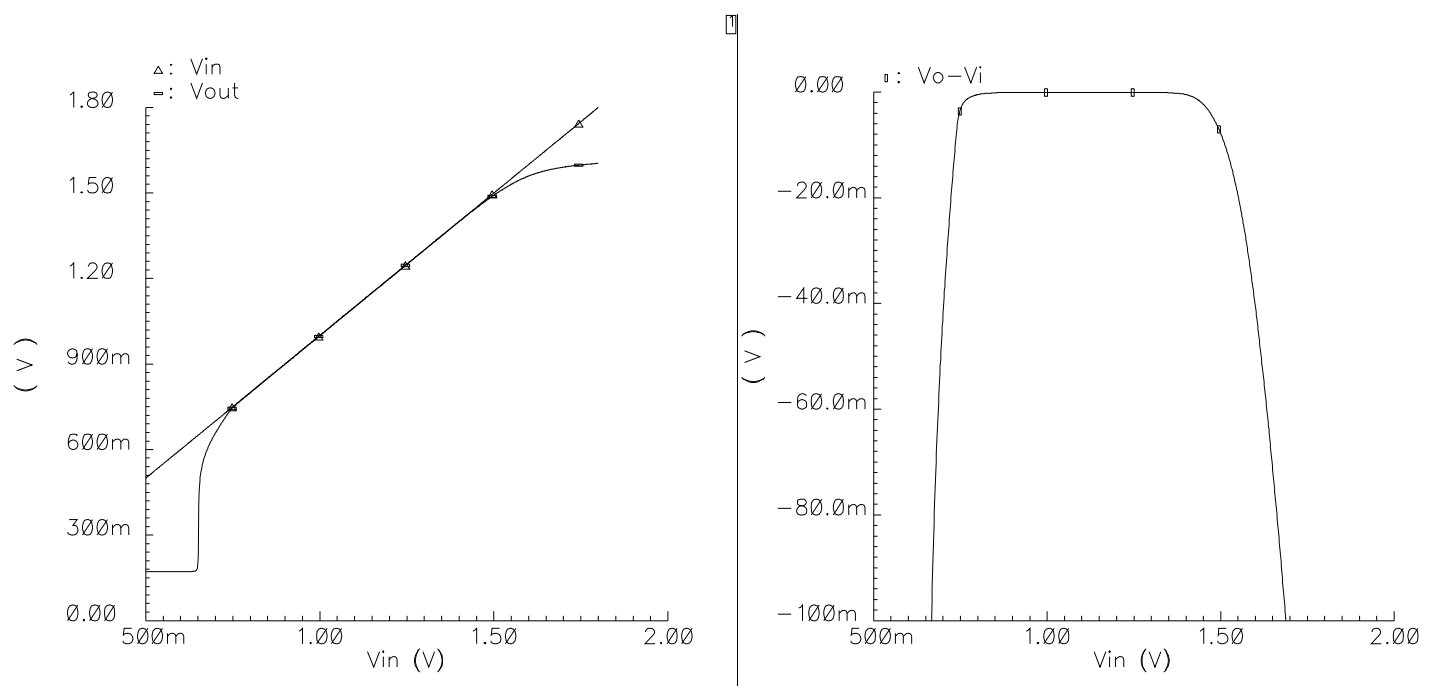

Figure 2-42 dc characteristics of the analog signal driver.

Figure 2-42 shows the de characteristics of the circuit. The $1 \%$ dc gain-variation range is about $570 \mathrm{mV}$ at $1.8-\mathrm{V}$ supply, and $V_{\text {out }}$ tracks $V_{\text {in }}$ closely. To examine the linearity from another aspect, Fig. 2-43 shows the IIP3 of the circuit, which is 32 $\mathrm{dBVp}$ or $39.5 \mathrm{Vp}$, a very high number for a CMOS circuit at $1.8 \mathrm{~V}$.

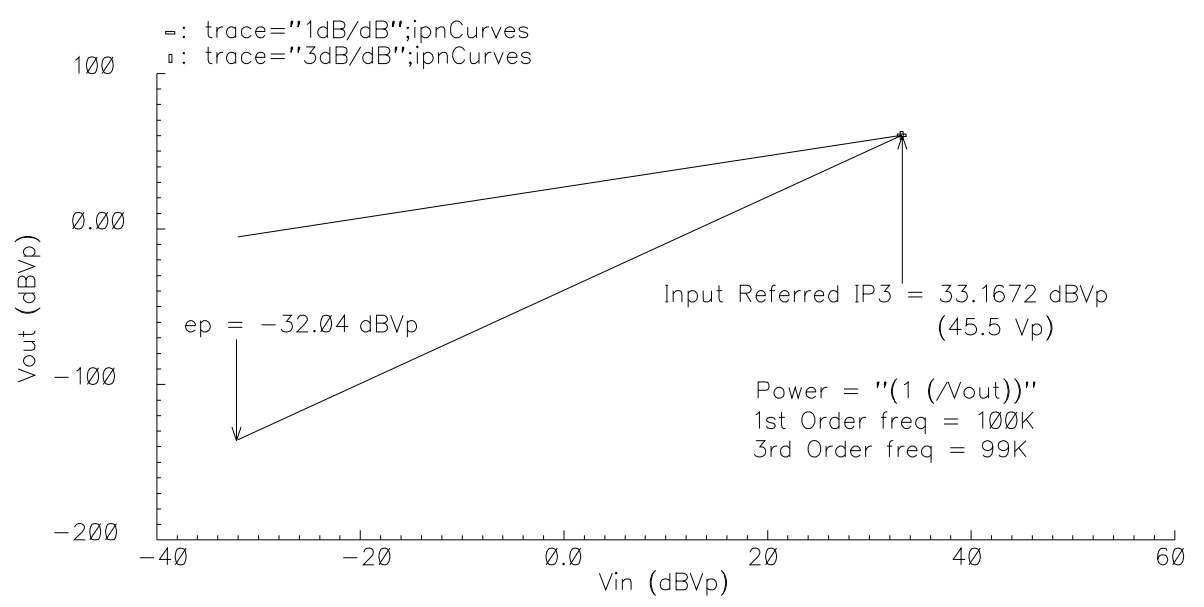

Figure 2-43 The IIP3 of the circuit is $33 \mathrm{dBVp}$ or $45.5 \mathrm{Vp}$, at $1.8 \mathrm{~V}$ power supply, single-ended. 

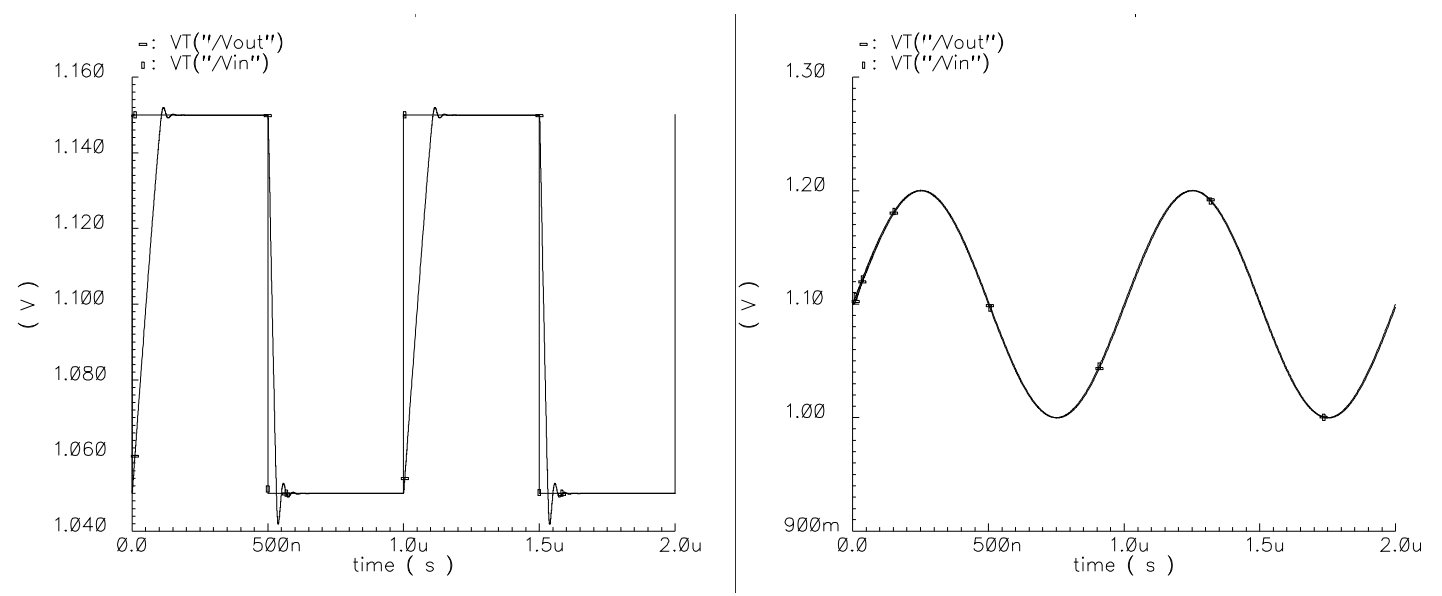

Figure 2-44 Transient simulation of the circuit, sinusoidal and pulse input.

Figure 2-44 shows the transient simulations of the circuit, for a sinusoidal input and a pulse input, both at $1 \mathrm{MHz}$. The sinusoidal output is almost an exact replica of the input; the rise time of the pulse output, however, is limited by the circuit's slew rate,

$$
S R_{\text {rising }}=\frac{d V_{\text {out }}}{d t}=\frac{I_{3}}{C_{\mathrm{L}}}
$$

The slew rate can be increased by increasing $I_{3}$ for a given load. $I_{1}\left(=I_{2}\right)$ can be kept unchanged to be more power efficient. The fall-time slew rate is primarily determined by the source-degeneration resistor $R_{\mathrm{S} 2}$, and unlike $S R_{\text {rising, }}$, is not constant with respect to $V_{\text {out}}$,

$$
S R_{\text {falling }}=\frac{d V_{\text {out }}}{d t} \approx \frac{V_{\text {out }} / R_{\mathrm{S} 2}}{C_{\mathrm{L}}}=\frac{V_{\text {out }}}{R_{\mathrm{S} 2} C_{\mathrm{L}}}
$$

The equation is exact when $V_{\text {out }}=V_{\text {OUT }}$. When $R_{\mathrm{S} 2}=0, S R_{\text {falling }}$ is indeed only limited by the intrinsic $R_{\mathrm{S}}$ and $R_{\mathrm{D}}$ of $M_{3}$ and could be very large. The sinusoidal signal is also subject to the slew rate limitation, of course, albeit at a higher amplitude.

To verify the circuit's robustness, Monte Carlo simulation results are shown in 
Fig. 2-45. The $6-\sigma$ gain variation range is only about $1.8 \mathrm{mdB}$, due to the dual feedback loops discussed above. The dc voltage mismatch, $V_{\mathrm{OUT}}-V_{\mathrm{IN}}$, is larger, with a 6- $\sigma$ range of $19 \mathrm{mV}$, i.e., $V_{\text {OUT }}$ could deviate as far as $V_{\mathrm{IN}} \pm 9.5 \mathrm{mV}$, due to the $V_{\mathrm{TH}}$ mismatch in $M_{1}$ and $M_{2}$, which differs from process to process. The mismatch can be reduced by increasing $M_{1}$ and $M_{2}$ 's $W$ and $L$ proportionally, with low impact to circuit $f_{\mathrm{p}}$, since $f_{\mathrm{p}}$ is mainly limited by $C_{\mathrm{L}}$, not the parasitic capacitors. The 6- $\sigma$ variation range of $f_{\mathrm{p}}$ is $30.6 \mathrm{MHz} \pm 0.57 \mathrm{MHz}$, or $3.7 \%$ of $f_{\mathrm{p}}$. The frequency profile of the gain is stable against process variations.

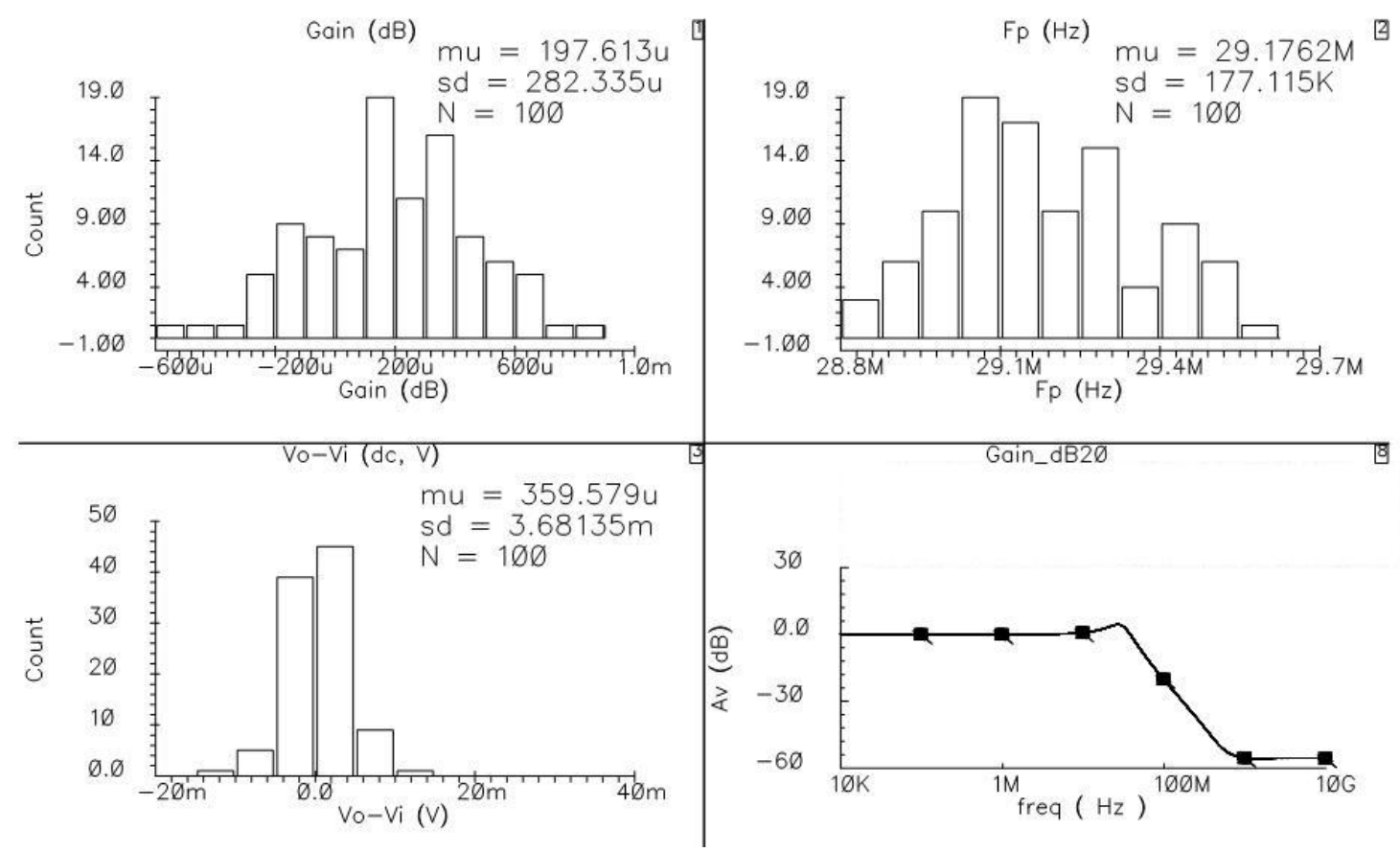

Figure 2-45 Monte Carlo simulation results.

\subsubsection{The all-NMOS-II Circuit Configured as an Active Inductor}

The all-NMOS-II circuit can be configured as an active inductor, as shown in Fig. 246 , by connecting the gate of $M_{2}$ to a common-mode voltage $V_{\mathrm{CM}}$, and using $V_{1}$ as 
input port. Per the previous analysis, $V_{2}$ replicates $V_{1}$, thus $M_{2}$ becomes a noninverting $G_{\mathrm{M}}$, with input at $V_{2}$ and output at $V_{3} . M_{3}$, degenerated by the optional $R_{\mathrm{S} 2}$, is an inverting $G_{\mathrm{M}}$, with input at $V_{3}$ and output at $V_{1}$. Thus a gyrator is obtained, converting the parasitic capacitor $C_{3}$ at node $V_{3}$ into an equivalent inductor at $V_{1}$, as shown by Fig. 2-47.

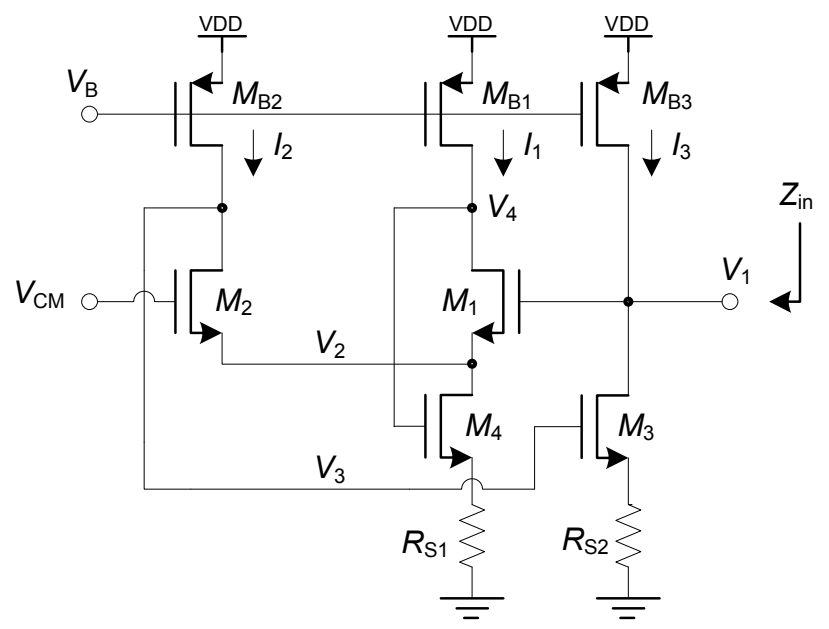

Figure 2-46 The all-NMOS-II circuit configured as an active inductor.

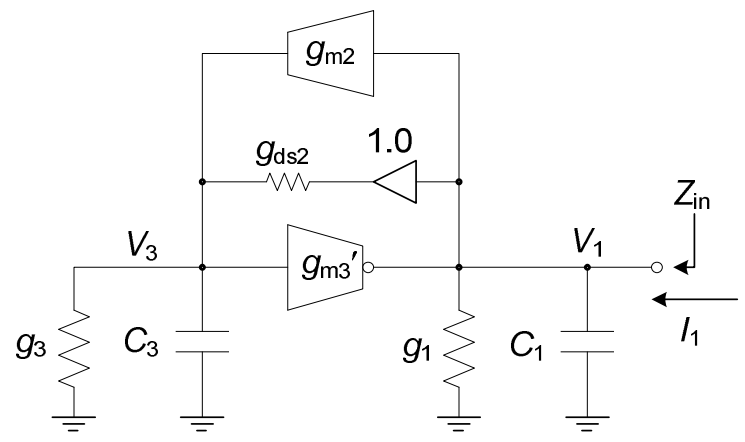

Figure 2-47 Small-signal equivalent circuit of the all-NMOS-II active inductor.

The small-signal elements are the same as in the above analysis. The input impedance $Z_{\text {in }}$ can be obtained through circuit analysis:

$$
Z_{\text {in }}(s)=\frac{V_{1}}{V_{1}}=\frac{\frac{s}{C_{1}}+\frac{g_{3}+g_{\mathrm{d} 2}}{C_{1} C_{3}}}{s^{2}+s\left(\frac{g_{1}}{C_{1}}+\frac{g_{\mathrm{ds} 2}+g_{3}}{C_{3}}\right)+\frac{\left(g_{\mathrm{m} 2}+g_{\mathrm{d} 2}\right) g_{\mathrm{m} 3}{ }^{\prime}+g_{1}\left(g_{\mathrm{d} 2}+g_{3}\right)}{C_{1} C_{3}}}
$$

Compared with the format of Eq. (2.7), $Z_{\text {in }}$ is recognized as the input impedance 
of an $R-L-C$ network, as shown in Fig. 2-27(b). At dc (s=0), we have

$$
r_{\mathrm{s}}=\frac{g_{3}+g_{\mathrm{ds} 2}}{\left(g_{\mathrm{m} 2}+g_{\mathrm{ds} 2}\right) g_{\mathrm{m} 3}{ }^{\prime}+g_{1}\left(g_{\mathrm{ds} 2}+g_{3}\right)} \approx \frac{g_{3}+g_{\mathrm{ds} 2}}{g_{\mathrm{m} 2} g_{\mathrm{m} 3}^{\prime}}
$$

As $s \rightarrow \infty$, we have

$$
\lim _{s \rightarrow \infty} Z_{\text {in }}(s)=\frac{1}{s C_{1}}
$$

Hence the shunt capacitor is

$$
C_{\mathrm{P}}=C_{1}
$$

Using the pole (self-resonance) frequency,

$$
\omega_{\mathrm{R}}=\sqrt{\frac{\left(g_{\mathrm{m} 2}+g_{\mathrm{ds} 2}\right) g_{\mathrm{m} 3}^{\prime}+g_{1}\left(g_{\mathrm{ds} 2}+g_{3}\right)}{C_{1} C_{3}}} \approx \sqrt{\frac{g_{\mathrm{m} 2} g_{\mathrm{m} 3}^{\prime}}{C_{1} C_{3}}}
$$

the equivalent inductor can be calculated as

$$
L_{\mathrm{eq}}=\frac{1}{\omega_{\mathrm{R}}^{2} C_{1}}=\frac{C_{3}}{\left(g_{\mathrm{m} 2}+g_{\mathrm{ds} 2}\right) g_{\mathrm{m} 3}^{\prime}+g_{1}\left(g_{\mathrm{ds} 2}+g_{3}\right)} \approx \frac{C_{3}}{g_{\mathrm{m} 2} g_{\mathrm{m} 3}^{\prime}}
$$

The quality factor at $\omega_{\mathrm{R}}$ is

$$
Q_{\mathrm{R}}=\frac{\omega_{\mathrm{R}}}{\frac{g_{1}}{C_{1}}+\frac{g_{\mathrm{ds} 2}+g_{3}}{C_{3}}}
$$

Using Eq. (2.8), the shunt resistance is calculated as

$$
R_{\mathrm{p}} \approx \frac{1}{g_{1}+\frac{C_{1}}{C_{3}}\left(g_{\mathrm{ds} 2}+g_{3}\right)}
$$

To appreciate the higher $\omega_{\mathrm{R}}$ of the all-NMOS-II active inductor, we compare Eqs. (2.107) and (2.55), and find that the all-NMOS-II circuit's $f_{\mathrm{R}}$ is 1.414 times that of the all-NMOS circuit. The reason is that due to the local feedback introduced by $M_{4}$ 
in Fig. 2-45, $V_{2}$ is no longer approximately $0.5 V_{1}$, but equal to $V_{1}$. Thus, the noninverting transconductor, $G_{\mathrm{M} 1}$, implemented by $M_{2}$, is doubled. Note that this frequency enhancement does not increase the circuit's power dissipation, because $M_{4}$ will exist as a biasing FET in the circuit anyway. The insertion of $M_{\mathrm{B} 1}$ does not require extra current or a $V_{\mathrm{dd}}$ increase; it merely shares part of the more than sufficient $V_{\mathrm{ds}}$ of $M_{1}$ in the all-NMOS circuit.

This voltage segmentation technique is sometimes dubbed "current recycling" in the industry. From another aspect, $V_{\mathrm{DD}}$ and $I_{\mathrm{DD}}$ are both resources, and can be broken down into a matrix, as shown in Fig. 2-48. The more devices we can fit into a given dimension set by $V_{\mathrm{DD}}$ and $I_{\mathrm{DD}}$, the more functionality and/or performance we can potentially obtain. The vertical size of the matrix is determined by $V_{\mathrm{DD}}$ and device $V_{\mathrm{TH}} \mathrm{S}$. The horizontal size is determined by $I_{\mathrm{DD}}$ and specific technology. The current per column cannot be infinitely reduced, when technology constraints, parasitic capacitance, and device matching are considered. Of course, we should not add a device just to "patch a hole" in the matrix without serving a useful purpose, as it is going to introduce parasitic elements and degrade the circuit performance. However, by inspecting a circuit for "holes" in the matrix, we can quickly locate places where a device may be introduced without increasing $I_{\mathrm{DD}}$.

As a very important side benefit obtained from the insertion of $M_{\mathrm{B} 1}$, the drain voltages of $M_{1}$ and $M_{2}$ are now equal, and this is the key reason for the high linearity of the circuit when used as an analog signal driver. 


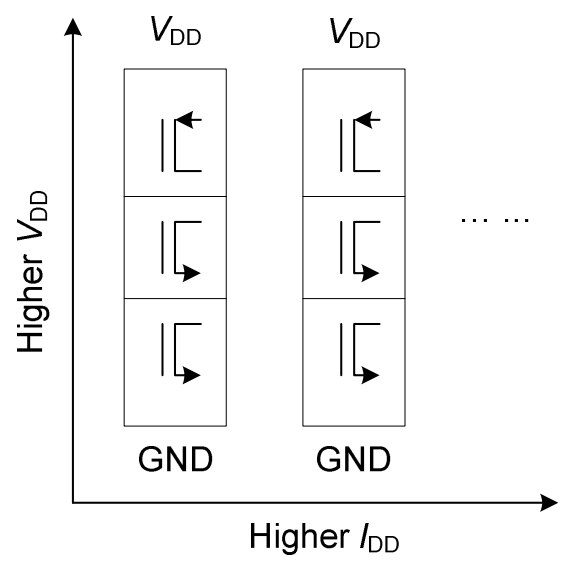

Figure 2-48 Lateral and vertical development of analog circuits.

\subsubsection{Example of the all-NMOS-II Active Inductor Circuit}

An all-NMOS-II active inductor example is shown in Fig. 2-49. All MOSFETs are kept at the same operating point, but the 4-pF load capacitor is separated into two parts at $V_{1}$ and $V_{3}$. The ac simulation results are shown in Fig. 2-50.

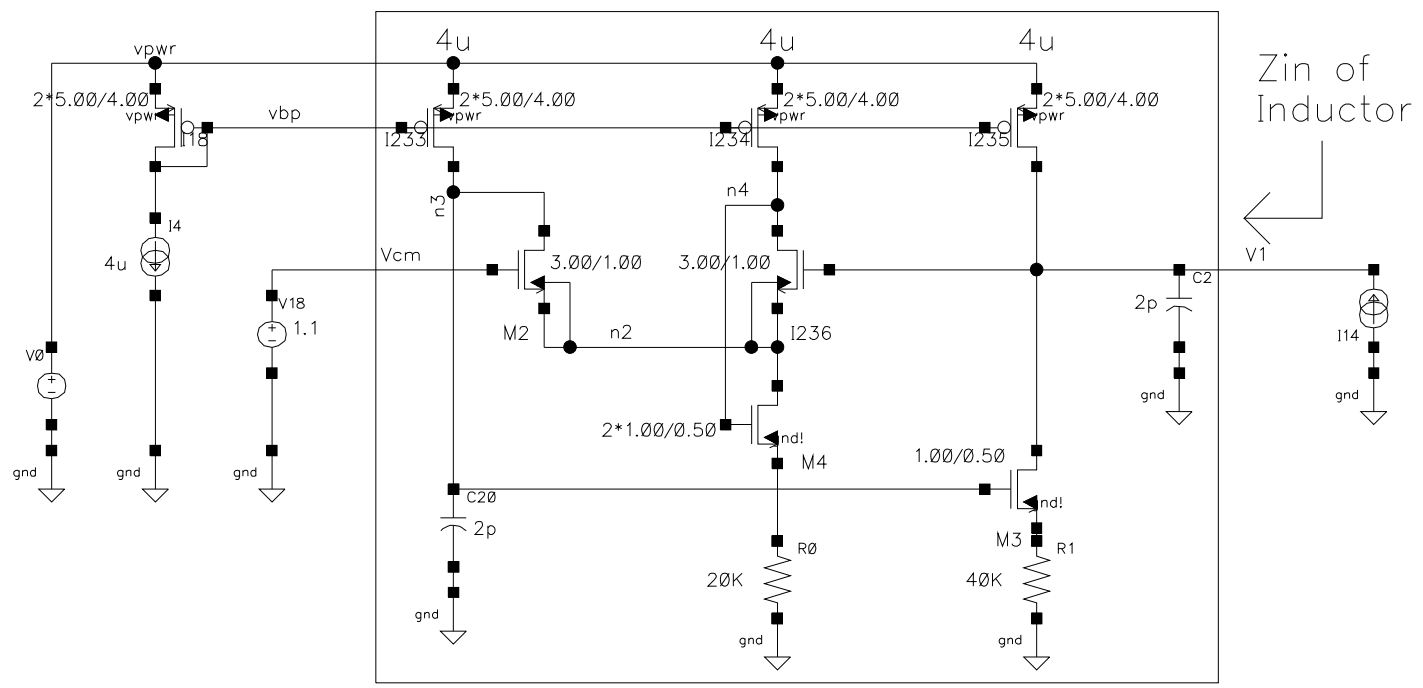

Figure 2-49 The all-NMOS-II circuit configured as an active inductor. 

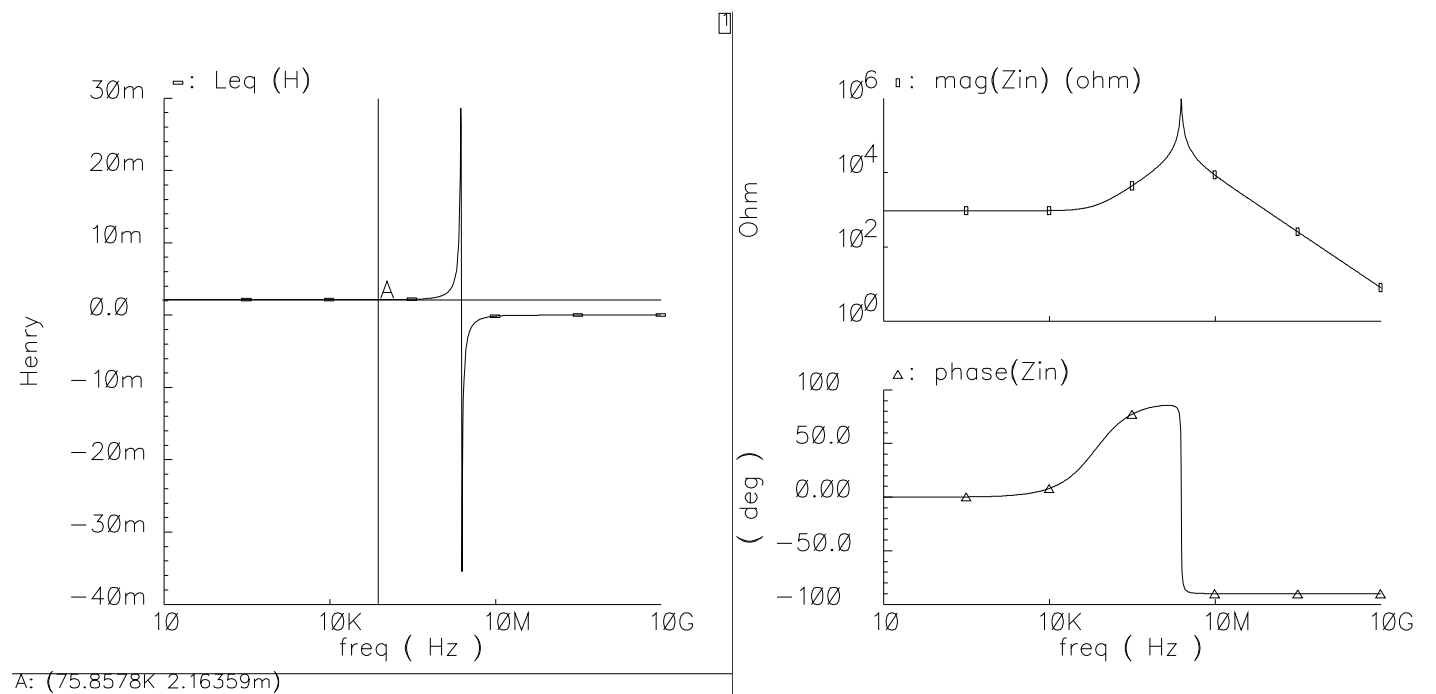

Figure 2-50 Example ac simulation results of the all-NMOS-II active inductor.

The component values of the equivalent circuit are extracted from the operating point: $g_{\mathrm{m} 2}=65.32 \mu \mathrm{S}, g_{\mathrm{m} 3}=51.96 \mu \mathrm{S}, C_{1}=2.017 \mathrm{pF}, C_{3}=2.007 \mathrm{pF}, g_{\mathrm{ds} 2}=1.11 \mu \mathrm{S}$, $g_{3}=80 \mathrm{nS}, g_{1}=0.245 \mu \mathrm{S}, R_{\mathrm{S} 2}=40 \mathrm{k} \Omega$. Using the above equations, the circuit parameters are calculated as $f_{\mathrm{R}}=2.65 \mathrm{MHz}, Q_{\mathrm{R}}=23.3, L_{\mathrm{eq}}=1.79 \mathrm{mH}, r_{\mathrm{s}}=1.06 \mathrm{k} \Omega$, very close to the simulated results shown in Fig. 2-50. The results are summarized in Table 2-4.

It is noted that when $C_{1}$ and $C_{3}$ are reduced to the parasitic capacitance of the devices and layout in order to obtain very high operating frequencies, the design for a certain $Q_{\mathrm{R}}$ becomes less straightforward than the above equations appear to indicate, because the parasitic pole and zero frequencies of the $V_{1} \rightarrow V_{2}$ transfer function of the $M_{4}$ and $M_{1}$ local feedback loop, as given by Eq. (2.91), become close to the circuit $f_{\mathrm{R}}$, and the phase shift of the noninverting $G_{\mathrm{M}}$ formed by $M_{2}-M_{1}-M_{4}$ modifies $Q_{\mathrm{R}}$ per section 2.5.3, Eq. (2.37). 
Table 2-4 Calculated versus Simulated Results for the All-NMOS-II circuit when used as an active inductor

\begin{tabular}{|c|c|c|c|c|c|c|c|c|}
\hline Parameter & Unit & Value & Equation & Parameter & Unit & Sims & Calc'ed & Equation \\
\hline$g_{\mathrm{m} 1}$ & uS & 65.31 & & $f_{\mathrm{R}}$ & $\mathrm{MHz}$ & 2.42 & 2.65 & $(2.107)$ \\
\hline$g_{\mathrm{m} 2}$ & $\mathrm{uS}$ & 65.32 & & $Q_{\mathrm{R}}$ & & 22.00 & 23.30 & $(2.109)$ \\
\hline$g_{\mathrm{m} 3}$ & $\mathrm{uS}$ & 51.96 & & $L_{\mathrm{eq}}$ & $\mathrm{mH}$ & 2.16 & 1.79 & $(2.108)$ \\
\hline$C_{1}$ & $\mathrm{pF}$ & 2.017 & $(2.98)$ & $r_{\mathrm{s}}$ & $\mathrm{kOhm}$ & 0.940 & 1.061 & $(2.104)$ \\
\hline$C_{3}$ & $\mathrm{pF}$ & 2.007 & $(2.97)$ & $R_{\mathrm{p}}$ & MOhm & 0.759 & 0.694 & $(2.110)$ \\
\hline$g_{\mathrm{ds} 2}$ & uS & 1.11 & & & & & & \\
\hline$g_{\mathrm{ds}, \mathrm{MB} 1}$ & $\mathrm{nS}$ & 80 & & & & & & \\
\hline$g_{\mathrm{ds}, \mathrm{MB} 2}$ & $\mathrm{nS}$ & 80 & & & & & & \\
\hline$g_{\mathrm{ds}, \mathrm{MB} 3}$ & $\mathrm{nS}$ & 90 & & & & & & \\
\hline$g_{\mathrm{ds} 3}$ & uS & 0.48 & & & & & & \\
\hline$R_{\mathrm{S} 2}$ & $\mathrm{kOhm}$ & 40 & & & & & & \\
\hline$g_{1}$ & uS & 0.245 & $(2.98)$ & & & & & \\
\hline$g_{3}$ & $\mathrm{nS}$ & 80 & $(2.95)$ & & & & & \\
\hline$g_{\mathrm{m} 3}{ }^{\prime}$ & $\mathrm{uS}$ & 16.88 & $(2.94)$ & & & & & \\
\hline
\end{tabular}

\subsection{Summary of the Active-Inductor Circuits}

The active-inductor circuits discussed in this chapter are summarized in Table 2-5.

The circuits can be classified into two types: those that are best used as an inductor, such as the load of the limiting amplifier in optic receivers; and those that are best used as a resonator, in obtaining high- $Q$ bandpass filters or oscillators.

It shall be noted that in designing $\mathrm{GHz}$ filters, the circuits are not used to directly replace the inductors in prototype $L-C$ filters, as this method cannot fully exploit the high-frequency potential of the active-inductor circuits, but rather directly or indirectly as a filter, after adding the necessary circuitry. This will be discussed in more detail in Chapter 3. When used as inductors, the active-inductor circuits are typically used as a load in wide-band pulse amplifiers and oscillators to mimic passive inductors. 
Table 2-5 Summary of active-inductor circuits

\begin{tabular}{|c|c|c|c|c|c|c|c|}
\hline $\begin{array}{l}\text { Active } \\
\text { Inductor }\end{array}$ & Section & $Q_{\mathrm{L}}$ & $Q_{R}$ & $\begin{array}{l}\text { Good for } \\
\text { inductor }\end{array}$ & $\begin{array}{l}\text { Good for } \\
\text { resonator }\end{array}$ & typical application & Note \\
\hline $\begin{array}{l}\text { Gate-R and } \\
\text { Gate-R-II }\end{array}$ & 2.4 .1 & $\begin{array}{l}\text { low to } \\
\text { moderate }\end{array}$ & low & yes & no & $\begin{array}{l}\text { wideband pulse } \\
\text { amplifier }\end{array}$ & \\
\hline $\begin{array}{l}\text { Cascode } \\
\text { Gate-R }\end{array}$ & 2.4 .2 & $\begin{array}{l}\text { low to } \\
\text { moderate }\end{array}$ & low & yes & no & $\begin{array}{l}\text { wideband pulse } \\
\text { amplifier }\end{array}$ & \\
\hline CG-CS & 2.4 .3 & $\begin{array}{l}\text { moderate } \\
\text { to high }\end{array}$ & low & yes & no & $\begin{array}{l}\text { wideband pulse } \\
\text { amplifier }\end{array}$ & easy to bias \\
\hline $\begin{array}{l}\text { Cascode CG- } \\
\text { CS }\end{array}$ & 2.4 .4 & $\begin{array}{l}\text { moderate } \\
\text { to high }\end{array}$ & low & yes & no & $\begin{array}{l}\text { wideband pulse } \\
\text { amplifier }\end{array}$ & $\begin{array}{l}\text { derived from } \\
\text { CG-CS }\end{array}$ \\
\hline CS-CD & 2.4 .5 & $\begin{array}{l}\text { moderate } \\
\text { to high }\end{array}$ & low & yes & no & $\begin{array}{l}\text { wideband/"low-noise" } \\
\text { amplifier }\end{array}$ & \\
\hline $\begin{array}{l}\text { Cascode and } \\
\text { Regulated } \\
\text { Cascode }\end{array}$ & 2.4 .6 & $\begin{array}{l}\text { moderate } \\
\text { to high }\end{array}$ & low & yes & no & $\begin{array}{l}\text { wideband/"low-noise" } \\
\text { amplifier }\end{array}$ & $\begin{array}{l}\text { Derived from } \\
\text { CS-CD, } \\
\text { easy to bias }\end{array}$ \\
\hline $\begin{array}{l}\text { Differential- } \\
\text { Pair }\end{array}$ & 2.4 .7 & $\begin{array}{l}\text { moderate } \\
\text { to high }\end{array}$ & low & yes & yes & bandpass filters & $\begin{array}{l}\text { very difficult } \\
\text { to bias }\end{array}$ \\
\hline Karsilayan's & 2.4 .8 & $\begin{array}{l}\text { moderate } \\
\text { to high }\end{array}$ & high & yes & yes & $\begin{array}{l}\text { bandpass/lowpass } \\
\text { filters }\end{array}$ & easy to bias \\
\hline $\begin{array}{l}\text { All-NMOS } \\
\text { (new in this } \\
\text { work) }\end{array}$ & 2.7 & $\begin{array}{l}\text { moderate } \\
\text { to high }\end{array}$ & high & yes & yes & $\begin{array}{l}\text { bandpass/lowpass } \\
\text { filters }\end{array}$ & $\begin{array}{l}\text { all-NMOS } \\
\text { signal path, } \\
\text { high } f_{\mathrm{R}} \text {, easy } \\
\text { to bias }\end{array}$ \\
\hline $\begin{array}{l}\text { all-NMOS-II } \\
\text { (new in this } \\
\text { work) }\end{array}$ & 2.8 & $\begin{array}{l}\text { moderate } \\
\text { to high }\end{array}$ & high & yes & yes & $\begin{array}{l}\text { analog signal driver, } \\
\text { voltage follower, } \\
\text { lowpass filter }\end{array}$ & $\begin{array}{l}\text { very high } \\
\text { linearity }\end{array}$ \\
\hline
\end{tabular}




\section{CHAPTER 3 \\ DESIGN OF RF ANALOG FILTERS}

\subsection{Designing RF Analog Filters with Active Inductors}

Analog filters can be constructed using active inductors and capacitors in ladder structures [10]. Since the active inductors are used as inductors, the filters will have to operate at frequencies well below (1-10\%) the inductor self-resonance frequency $f_{\mathrm{R}}$, so that the active inductors will behave as inductors rather than resonators. To obtain RF filters that operate near or at $f_{\mathrm{R}}$, we will have to treat the active inductor as a resonator and use different methods.

\subsubsection{Bandpass Filters}

If floating active inductors are available, bandpass filters can be obtained by replacing the passive inductors in an $L-C$ ladder bandpass filter with active inductors [10]. However, the bandpass filter's center frequency $f_{0}$ has to be much lower than the active inductors' self-resonance frequency $f_{\mathrm{R}}$, because the active inductor will behave like an inductor only at frequencies much lower than $f_{\mathrm{R}}$. To obtain the highest possible $f_{0}$, we may use the active inductor as a resonator, as shown in Fig. 3-1, where the $R-L-$ $C$ network is the equivalent circuit of an active inductor. 


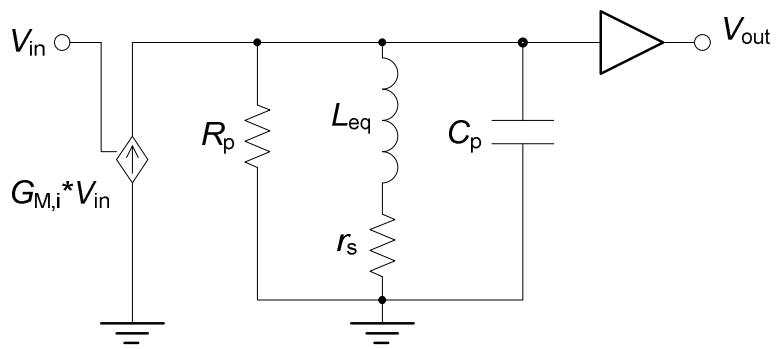

Figure 3-1 Constructing an HF active-inductor bandpass filter.

From Section 2.2 we know that the input impedance $Z_{\text {in }}$ of an active inductor has a bandpass profile when $Q_{\mathrm{R}}$ is high $(>2)$ and $r_{\mathrm{s}}$ 's effect at dc is ignored. Thus, when an input current is applied to $Z_{\text {in }}$, the voltage across the $Z_{\text {in }}$ port will have a bandpass profile, too. A transconductor $G_{\mathrm{M}, \mathrm{i}}$ can be used to convert $V_{\text {in }}$ into a current. A voltage follower is needed at the output to drive the resistive/capacitive load, preventing it from reducing the active inductor's $f_{\mathrm{R}}$ and/or $Q_{\mathrm{R}}$. Real transconductors inevitably have an output capacitance $C_{\mathrm{o}}$ and leakage $g_{\mathrm{o}}$, and the voltage follower has an input capacitance $C_{\mathrm{i}}$. $C_{\mathrm{i}}$ and $C_{\mathrm{o}}$ can be absorbed by $C_{\mathrm{p}}$, reducing $f_{\mathrm{R}}$ slightly, and $g_{\mathrm{o}}$ can be absorbed by $R_{\mathrm{p}}$, reducing $Q_{\mathrm{R}}$. Therefore, the parasitic elements of $G_{\mathrm{M}, \mathrm{i}}$ and the output buffer do not change the order or the function format of $Z_{\text {in }}$, but merely change its parameters to some extent. If the active inductor is designed with these parasitic elements included, the desired frequency and $Q$ can be obtained.

Assuming that $f_{\mathrm{R}}$ becomes $f_{0}$ and $Q_{\mathrm{R}}$ becomes $Q_{0}$ after taking into account the parasitic elements, the bandpass filter transfer function is

$$
T(s)=\frac{V_{\text {out }}}{V_{\text {in }}}=G_{\mathrm{M}, \mathrm{i}} Z_{\text {in }}=\frac{G_{\mathrm{M}, \mathrm{i}} \omega_{0}^{2}}{s^{2}+s \frac{\omega_{0}}{Q_{0}}+\omega_{0}^{2}}
$$

where the effect of $r_{\mathrm{s}}$ has been neglected. Including $r_{\mathrm{s}}, T(0)=G_{\mathrm{M}, \mathrm{i}}{ }^{*} r_{\mathrm{s}} \neq 0$, which can be neglected when $Q_{\mathrm{R}}$ is very high. The mid-band gain of the bandpass filter is 


$$
H_{\mathrm{M}}=G_{\mathrm{M}, \mathrm{i}}\left(R_{\mathrm{p}} \| g_{\mathrm{o}}\right)
$$

In practice, $G_{\mathrm{M}, \mathrm{i}}$ may be implemented by a single FET or a differential pair; and the output buffer may be implemented by a source follower.

High-order filters may be constructed by cascading several of the $2^{\text {nd }}$-order bandpass filters shown in Fig. 3-1. For example, by tuning the $f_{0}$ and $Q_{0}$ of three filters properly, a maximally flat passband may be obtained. However, there are two issues limiting its practicality:

1. Tuning $f_{0}$ and $Q_{0}$ of several filters together is difficult at a few $\mathrm{GHz}$, if possible at all;

2. Due to the feed-through capacitances, such as $C_{\mathrm{gd}}$ of $G_{\mathrm{M}, \mathrm{i}}$, as well as substrate coupling at several $\mathrm{GHz}$, the maximum stopband attenuation is going to be limited to $30-50 \mathrm{~dB}$, depending on the particular design and operating frequency. Thus, the higher stopband attenuation benefit of a higher-order filter will be defeated.

It may be possible to design a flat passband with cascaded low- $Q$ bandpass filters and at low frequencies (e.g., $<100 \mathrm{MHz}$ ). The resulting filter cannot be narrowband, of course.

\subsubsection{Lowpass Filters}

Similar to the bandpass filter, if floating active inductors are available lowpass filters can be constructed by replacing the passive inductors in an $L-C$ lowpass filter with active inductors. The lowpass filter's cutoff frequency $f_{\mathrm{c}}\left(\approx f_{\mathrm{p}}\right)$ must be much less than 
$f_{\mathrm{R}}$ of the active inductors. To obtain a lowpass filter with the highest possible $f_{\mathrm{p}}$, we must use the active inductor directly.

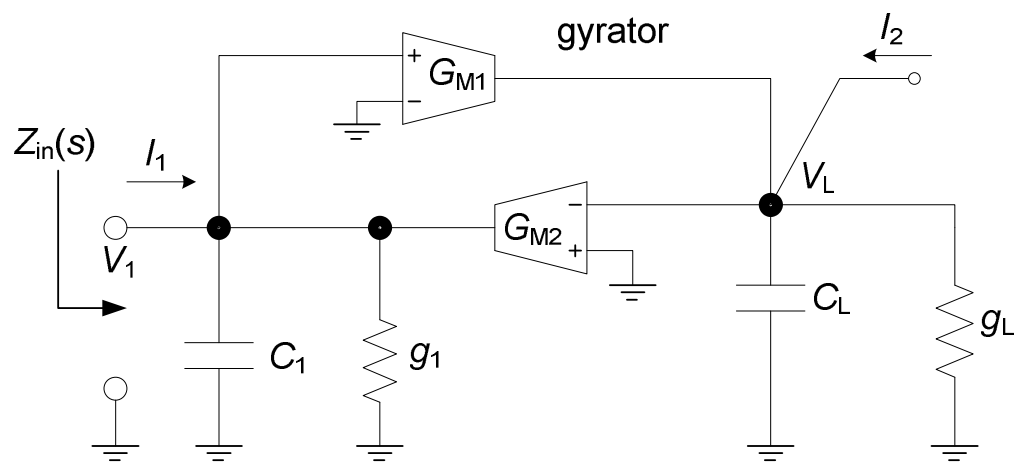

Figure 3-2 Implementing a lowpass filter using the active inductor.

The lowpass function is in fact already provided by the gyrator used to implement the active inductor, as shown in Fig. 3-2. This will be obvious after the following discussion. For the circuit in Fig. 3-2, if $I_{2}=0$, and an input current $I_{1}$ is injected into node $V_{1}$, using circuit analysis methods, the trans-impedance transfer function may be obtained as

$$
T(s)=\frac{V_{\mathrm{L}}}{I_{1}}=\frac{\frac{G_{\mathrm{M} 1}}{C_{1} C_{\mathrm{L}}}}{s^{2}+s\left(\frac{g_{\mathrm{L}}}{C_{\mathrm{L}}}+\frac{g_{1}}{C_{1}}\right)+\frac{G_{\mathrm{M} 1} G_{\mathrm{M} 2}+g_{1} g_{\mathrm{L}}}{C_{1} C_{\mathrm{L}}}}=\frac{H_{0} \omega_{\mathrm{p}}^{2}}{s^{2}+s \frac{\omega_{\mathrm{p}}}{Q_{\mathrm{p}}}+\omega_{\mathrm{p}}^{2}}
$$

which is a lowpass function. The transfer function parameters are

$$
\begin{aligned}
& H_{0}=\frac{G_{\mathrm{M} 1}}{G_{\mathrm{M} 1} G_{\mathrm{M} 2}+g_{1} g_{\mathrm{L}}} \approx \frac{1}{G_{\mathrm{M} 2}}, \quad \omega_{\mathrm{p}}=\sqrt{\frac{G_{\mathrm{M} 1} G_{\mathrm{M} 2}+g_{1} g_{\mathrm{L}}}{C_{1} C_{\mathrm{L}}}} \approx \sqrt{\frac{G_{\mathrm{M} 1} G_{\mathrm{M} 2}}{C_{1} C_{\mathrm{L}}}}=\omega_{\mathrm{R}} \\
& Q_{\mathrm{p}}=\frac{\omega_{\mathrm{p}}}{\frac{g_{\mathrm{L}}}{C_{\mathrm{L}}}+\frac{g_{1}}{C_{1}}}=Q_{\mathrm{R}}
\end{aligned}
$$

In other words, the pole frequency $f_{\mathrm{p}}$ and $Q_{\mathrm{p}}$ of the lowpass filter are the same as the self-resonance frequency $f_{\mathrm{R}}$ and $Q_{\mathrm{R}}$ of the active inductor. $T(s)$ is recognized as a $2^{\text {nd }}$-order function with conjugate poles, and has the advantage of wider bandwidth 
with an appropriate $Q_{\mathrm{p}}$ (e.g., between 0.707 and 2) compared with a $1^{\text {st }}$-order filter or a cascade of $1^{\text {st }}$-order sections. This is equivalent to the "shunt peaking" bandwidth enhancement technique $[16,83]$ when a transistor is loaded with a capacitor, without the need for a passive inductor.

Alternatively, if $I_{1}=0$, and $I_{2}$ is the input current, it can be found that the transimpedance transfer function

$$
T(s)=\frac{V_{1}}{I_{2}}=\frac{-\frac{G_{\mathrm{M} 2}}{C_{1} C_{\mathrm{L}}}}{s^{2}+s\left(\frac{g_{\mathrm{L}}}{C_{\mathrm{L}}}+\frac{g_{1}}{C_{1}}\right)+\frac{G_{\mathrm{Ml}} G_{\mathrm{M} 2}+g_{1} g_{\mathrm{L}}}{C_{1} C_{\mathrm{L}}}}=\frac{H_{0} \omega_{\mathrm{p}}^{2}}{s^{2}+s \frac{\omega_{\mathrm{p}}}{Q_{\mathrm{p}}}+\omega_{\mathrm{p}}^{2}}
$$

is also a lowpass function, but with inverting gain. The transfer function parameters are

$$
\begin{aligned}
& H_{0}=\frac{-G_{\mathrm{M} 2}}{G_{\mathrm{M} 1} G_{\mathrm{M} 2}+g_{1} g_{\mathrm{L}}} \approx-\frac{1}{G_{\mathrm{M} 1}}, \omega_{\mathrm{p}}=\sqrt{\frac{G_{\mathrm{M} 1} G_{\mathrm{M} 2}+g_{1} g_{\mathrm{L}}}{C_{1} C_{\mathrm{L}}}} \approx \sqrt{\frac{G_{\mathrm{M} 1} G_{\mathrm{M} 2}}{C_{1} C_{\mathrm{L}}}}=\omega_{\mathrm{R}} \\
& Q_{\mathrm{p}}=\frac{\omega_{\mathrm{p}}}{\frac{g_{\mathrm{L}}}{C_{\mathrm{L}}}+\frac{g_{1}}{C_{1}}}=Q_{\mathrm{R}}
\end{aligned}
$$

The two lowpass functions are dual functions of each other, and there is no reason to favor one over the other.

To utilize the lowpass trans-impedance functions, we may expect the need of an input transconductor to convert the input voltage into a current. However, in many cases, during transistor-level circuit design, by some clever circuit techniques, it is possible to free one of the grounded terminals of $G_{\mathrm{M} 1}$ or $G_{\mathrm{M} 2}$ in Fig. 3-2 and use it as the input terminal, thus eliminate the need for an input $G_{\mathrm{M}}$. An example is shown in Fig. 3-3. The transfer function is 


$$
T(s)=\frac{V_{1}}{V_{\text {in }}}=\frac{\frac{G_{\mathrm{M} 1} G_{\mathrm{M} 2}}{C_{1} C_{\mathrm{L}}}}{s^{2}+s\left(\frac{g_{\mathrm{L}}}{C_{\mathrm{L}}}+\frac{g_{1}}{C_{1}}\right)+\frac{G_{\mathrm{M} 1} G_{\mathrm{M} 2}+g_{1} g_{\mathrm{L}}}{C_{1} C_{\mathrm{L}}}}=\frac{H_{0} \omega_{\mathrm{p}}^{2}}{s^{2}+s \frac{\omega_{\mathrm{p}}}{Q_{\mathrm{p}}}+\omega_{\mathrm{p}}^{2}}
$$

with transfer function parameters being

$$
\begin{aligned}
& H_{0}=\frac{G_{\mathrm{M} 1} G_{\mathrm{M} 2}}{G_{\mathrm{M} 1} G_{\mathrm{M} 2}+g_{1} g_{\mathrm{L}}} \approx 1, \omega_{\mathrm{p}}=\sqrt{\frac{G_{\mathrm{M} 1} G_{\mathrm{M} 2}+g_{1} g_{\mathrm{L}}}{C_{1} C_{\mathrm{L}}}} \approx \sqrt{\frac{G_{\mathrm{M} 1} G_{\mathrm{M} 2}}{C_{1} C_{\mathrm{L}}}}=\omega_{\mathrm{R}} \\
& Q_{\mathrm{p}}=\frac{\omega_{\mathrm{p}}}{\frac{g_{\mathrm{L}}}{C_{\mathrm{L}}}+\frac{g_{1}}{C_{1}}}=Q_{\mathrm{R}}
\end{aligned}
$$

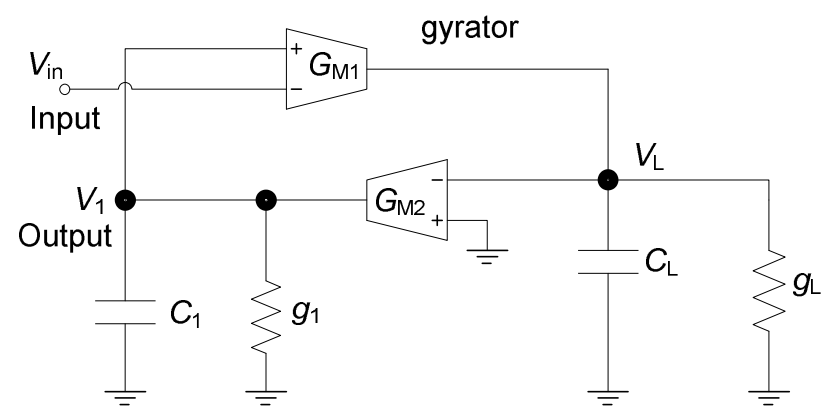

Figure 3-3 Reusing $G_{\mathrm{M} 1}$ as input $G_{\mathrm{M}}$ to implement a lowpass filter.

This is a very efficient circuit structure, which has been used in the all-NMOS-II analog signal driver circuit in Chapter 2.

\subsection{The RF Bandpass Filter in this Work}

\subsubsection{The Bandpass Filter Circuit}

The high- $Q$ bandpass filter circuit is shown in Fig. 3-4. The right half side is recognized as the active-inductor circuit, whose input impedance has a bandpass frequency profile. Transistor $M_{\mathrm{i}}$ converts the input voltage, $V_{\mathrm{in}}$, into a current, and applies it to the inductor port. The output voltage is taken from that port as $V_{\text {out. }}$ A very-small-size source follower connects to $V_{\text {out }}$, to isolate the load and reduce the stray capacitance from interconnects. 


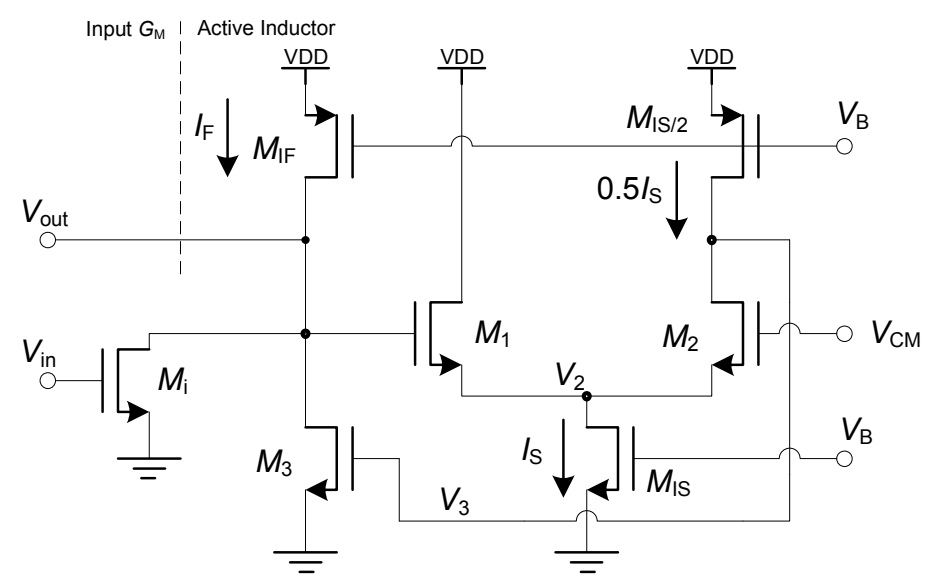

Figure 3-4 The bandpass filter circuit.

The transfer function is derived by multiplying the transconductance of $M_{\mathrm{i}}, G_{\mathrm{M}, \mathrm{i}}$, with $Z_{\text {in }}$ of the active inductor (Eq. 2.52)

$$
T(s)=\frac{G_{\mathrm{M}, \mathrm{i}}\left(\frac{s}{C_{1}}+\frac{g_{3}}{C_{1} C_{3}}\right)}{s^{2}+s\left(\frac{g_{1}}{C_{1}}+\frac{g_{3}}{C_{3}}+\frac{g_{1} g_{3} C_{2}}{C_{1} C_{3} G}-\omega^{2} \frac{C_{2}}{G}\right)+\frac{g_{\mathrm{m} 2}}{G} \frac{g_{\mathrm{m} 1 g_{\mathrm{m} 3}}}{C_{1} C_{3}}}
$$

The transfer-function parameters are

$$
\begin{aligned}
& H_{\mathrm{M}}=\frac{G_{\mathrm{M}, \mathrm{i}}}{g_{1}+\frac{C_{1}}{C_{3}} g_{3}+\frac{C_{2} g_{1} g_{3}}{C_{3} G}-\omega^{2} \frac{C_{1} C_{2}}{G}} \approx G_{\mathrm{M}, \mathrm{i}} R_{\mathrm{p}}, \quad \omega_{0}=\sqrt{\frac{g_{\mathrm{m} 1} g_{\mathrm{m} 2} g_{\mathrm{m} 3}}{G C_{1} C_{3}}} \approx \sqrt{\frac{0.5 g_{\mathrm{m} 1} g_{\mathrm{m} 3}}{C_{1} C_{3}}} \\
& Q_{0}=\frac{\sqrt{g_{\mathrm{m} 1} g_{\mathrm{m} 2} g_{\mathrm{m} 3} /\left(G C_{1} C_{3}\right)}}{\frac{g_{1}}{C_{1}}+\frac{g_{3}}{C_{3}}-\frac{C_{2}}{G}\left(\omega_{0}^{2}-\frac{g_{1} g_{3}}{C_{1} C_{3}}\right)}=\frac{\omega_{0}}{\frac{g_{1}}{C_{1}}+\frac{g_{3}}{C_{3}}-\frac{C_{2}}{G}\left(\omega_{0}^{2}-\frac{g_{1} g_{3}}{C_{1} C_{3}}\right)} \\
& H_{0}=T(0)=\frac{G_{\mathrm{M}, \mathrm{i}} g_{3} G}{g_{\mathrm{m} 1} g_{\mathrm{m} 2} g_{\mathrm{m} 3}} \approx \frac{G_{\mathrm{M}, \mathrm{i}} g_{3}}{0.5 g_{\mathrm{m} 1} g_{\mathrm{m} 3}} \neq 0
\end{aligned}
$$

Note that $C_{1}$ and $g_{1}$ should be adjusted to reflect the additional parasitic capacitance and conductance from $M_{\mathrm{i}}$ and the output buffer. Different from an ideal bandpass filter, the filter attenuation at dc is not infinity, but determined by the circuit loss $g_{3}$, which is transformed into the equivalent series resistor $r_{\mathrm{s}}$.

Transistor $M_{\mathrm{i}}$ draws current out of the active-inductor circuit for its own biasing, 
and $M_{\mathrm{IF}}$ needs to be augmented to allow this. The final circuit is designed in pseudodifferential form, and $M_{\mathrm{i}}$ together with its dual component becomes a common-source differential pair.

\subsubsection{Noise Analysis of the Bandpass Filter}

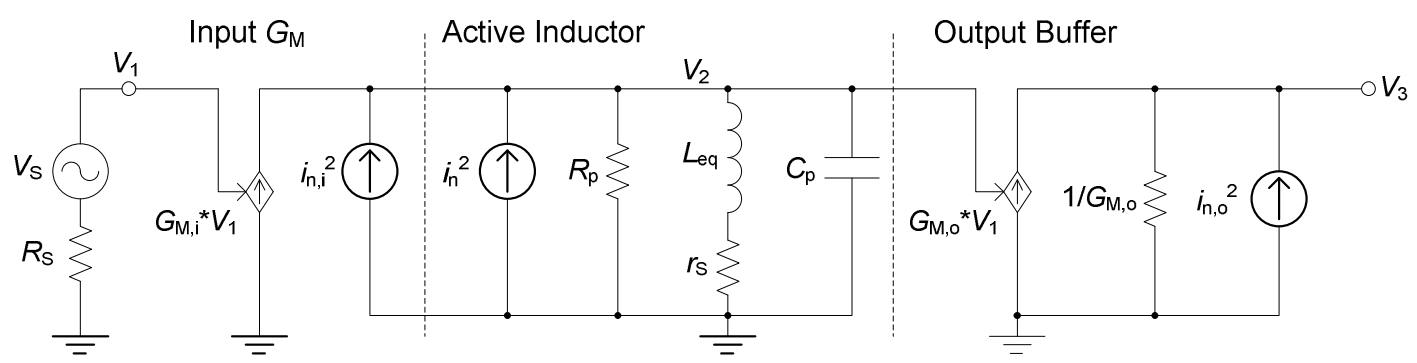

Figure 3-5 Small-signal equivalent circuit of the bandpass filter with noise elements.

Figure 3-5 shows the small-signal equivalent circuit of the bandpass filter with noise elements, where $i_{\mathrm{n}, \mathrm{i}}{ }^{2}$ and $i_{\mathrm{n}, \mathrm{o}}{ }^{2}$ represent the noise currents from the input $G_{\mathrm{M}}$ and output buffer, respectively, and $i_{\mathrm{n}}{ }^{2}$ is the equivalent noise current of the active inductor (Section 2.6). It would be easy to assume that $i_{\mathrm{n}, \mathrm{i}}{ }^{2}$ is $4 k T \Gamma_{\mathrm{n}} G_{\mathrm{M}, \mathrm{i}}$; however, we shall remember that by hooking $M_{\mathrm{i}}$ to the active inductor in Fig. 3-4, we have to increase $M_{\mathrm{IF}}$ accordingly so that the active inductor's biasing currents are not changed. In addition, per Eq. (2.67), for equal $V_{\text {od, }}$, a PFET and a NFET have the same $g_{\mathrm{m}}$ value when $I_{\mathrm{D}}$ is the same. Therefore, we have

$$
i_{\mathrm{n}, \mathrm{i}}^{2} \approx 4 k T \Gamma_{\mathrm{n}} G_{\mathrm{M}, \mathrm{i}}+4 k T \Gamma_{\mathrm{p}} G_{\mathrm{M}, \mathrm{i}}=4 k T\left(\Gamma_{\mathrm{n}}+\Gamma_{\mathrm{p}}\right) G_{\mathrm{M}, \mathrm{i}}
$$

The output buffer is modeled as $G_{\mathrm{M}, \mathrm{o}}$ driving a resistor of $1 / G_{\mathrm{M}, \mathrm{o}}$. The load capacitor at $V_{3}$ is ignored since the pole frequency of the output buffer is assumed to be much greater than the filter's $f_{0}$. Similarly, the noise current of the output buffer is twice that of the main FET due to the load current source, assuming $V_{\text {od }}$ is the same for 
both,

$$
i_{\mathrm{n}, \mathrm{o}}^{2} \approx 4 k T \Gamma_{\mathrm{n}} G_{\mathrm{M}, \mathrm{o}}+4 k T \Gamma_{\mathrm{n}} G_{\mathrm{M}, \mathrm{o}}=8 k T \Gamma_{\mathrm{n}} G_{\mathrm{M}, \mathrm{o}}
$$

At $f_{0}, j \omega L_{\mathrm{eq}}$ cancels $1 /\left(\omega C_{\mathrm{p}}\right)$. Ignoring $r_{\mathrm{s}}$, the filter gain is

$$
\left|T\left(\omega_{0}\right)\right|=G_{\mathrm{M}, \mathrm{i}} R_{\mathrm{p}}
$$

When the filter is driven by a port element of resistance $R_{\mathrm{S}}$, using Eq. (2.50), the total output noise voltage at $f_{0}$ is

$$
\begin{aligned}
& v_{\mathrm{n}, \mathrm{o}}^{2}=\left[\left(4 k T R_{\mathrm{S}} G_{\mathrm{M}, \mathrm{i}}^{2}+i_{\mathrm{n}, \mathrm{i}}^{2}+i_{\mathrm{n}}^{2}\right) R_{\mathrm{p}}^{2} G_{\mathrm{M}, \mathrm{o}}^{2}+i_{\mathrm{n}, \mathrm{o}}^{2}\right] \frac{1}{G_{\mathrm{M}, \mathrm{o}}^{2}} \\
& =\left[4 k T R_{\mathrm{S}} G_{\mathrm{M}, \mathrm{i}}^{2}+4 k T\left(\Gamma_{\mathrm{n}}+\Gamma_{\mathrm{p}}\right) G_{\mathrm{M}, \mathrm{i}}+4 k T\left(K_{\mathrm{NF} 1} G_{\mathrm{M} 1}+K_{\mathrm{NF} 2} G_{\mathrm{M} 2}\right)\right] R_{\mathrm{p}}^{2}+\frac{8 k T \Gamma_{\mathrm{n}}}{G_{\mathrm{M}, \mathrm{o}}}
\end{aligned}
$$

If only the noise from $R_{\mathrm{S}}$ is considered, the output noise becomes

$$
v_{\mathrm{n}, \mathrm{o}, \mathrm{Rs} \text { only }}^{2}=4 k T R_{\mathrm{S}} G_{\mathrm{M}, \mathrm{i}}^{2} R_{\mathrm{p}}^{2} G_{\mathrm{M}, \mathrm{o}}^{2} \frac{1}{G_{\mathrm{M}, \mathrm{o}}^{2}}=4 k T R_{\mathrm{S}} G_{\mathrm{M}, \mathrm{i}}^{2} R_{\mathrm{p}}^{2}
$$

Thus, the noise factor is

$$
\begin{aligned}
& N F=\frac{v_{\mathrm{n}, \mathrm{o}}^{2}}{v_{\mathrm{n}, \mathrm{R}, \mathrm{Rs} \text { only }}}=\frac{\left[4 k T R_{\mathrm{S}} G_{\mathrm{M}, \mathrm{i}}^{2}+4 k T\left(\Gamma_{\mathrm{n}}+\Gamma_{\mathrm{p}}\right) G_{\mathrm{M}, \mathrm{i}}+4 k T\left(K_{\mathrm{NF} 1} G_{\mathrm{M} 1}+K_{\mathrm{NF} 2} G_{\mathrm{M} 2}\right)\right] R_{\mathrm{p}}^{2}+\frac{8 k T \Gamma_{\mathrm{n}}}{G_{\mathrm{M}, \mathrm{o}}}}{4 k T R_{\mathrm{S}} G_{\mathrm{M}, \mathrm{i}}^{2} R_{\mathrm{p}}^{2}} \\
& =1+\frac{\left(\Gamma_{\mathrm{n}}+\Gamma_{\mathrm{p}}\right) G_{\mathrm{M}, \mathrm{i}}+\left(K_{\mathrm{NF} 1} G_{\mathrm{M} 1}+K_{\mathrm{NF} 2} G_{\mathrm{M} 2}\right)+\frac{2 \Gamma_{\mathrm{n}}}{G_{\mathrm{M}, 0} R_{\mathrm{p}}^{2}}}{R_{\mathrm{S}} G_{\mathrm{M}, \mathrm{i}}^{2}}
\end{aligned}
$$

For a given active inductor, increasing $G_{\mathrm{M}, \mathrm{i}}$ can reduce $N F$, at the cost of reducing the maximum input signal level, since $H_{\mathrm{M}}=G_{\mathrm{M}, \mathrm{i}} R_{\mathrm{p}}$.

Equation (3.17) is derived for a single-ended circuit. If the BPF circuit in Fig. 35 is built in a pseudo-differential structure, and $R_{\mathrm{S}}$ connects to the filter through a balun, the NF will be $3 \mathrm{~dB}$ higher than the single-ended circuit, since every squared 
noise source within the circuit is doubled (see also Section 2.7.3).

\subsubsection{Bandpass Filter Example}

Using Fig. 3-4, a BPF example is built based on the all-NMOS active inductor presented in Section 2.7, in a pseudo-differential structure, as shown in Fig. 3-6. A differential-pair input transconductor $G_{\mathrm{M}, \mathrm{i}}$ and a pair of source followers are added to form a bandpass filter per the previous discussion. The biasing conditions are identical to those of the active inductor shown in Fig. 2.29: $I_{\mathrm{M}_{\mathrm{IF}}}=I_{\mathrm{M}_{\mathrm{IS}}}=250 \mu \mathrm{A}, V_{\mathrm{F}}=V_{\mathrm{Q}}=0.6$ $\mathrm{V}, V_{\mathrm{CM}}=1.325 \mathrm{~V}, V_{\mathrm{DD}}=1.8 \mathrm{~V}$, and $I_{\mathrm{B}}=7 \mu \mathrm{A}$. The circuit simulation results are: $f_{0}=$ $6.4 \mathrm{GHz}, Q_{0}=90, H_{\mathrm{M}, V 2}=1.868, H_{\mathrm{M}, V 3}=1.362$.
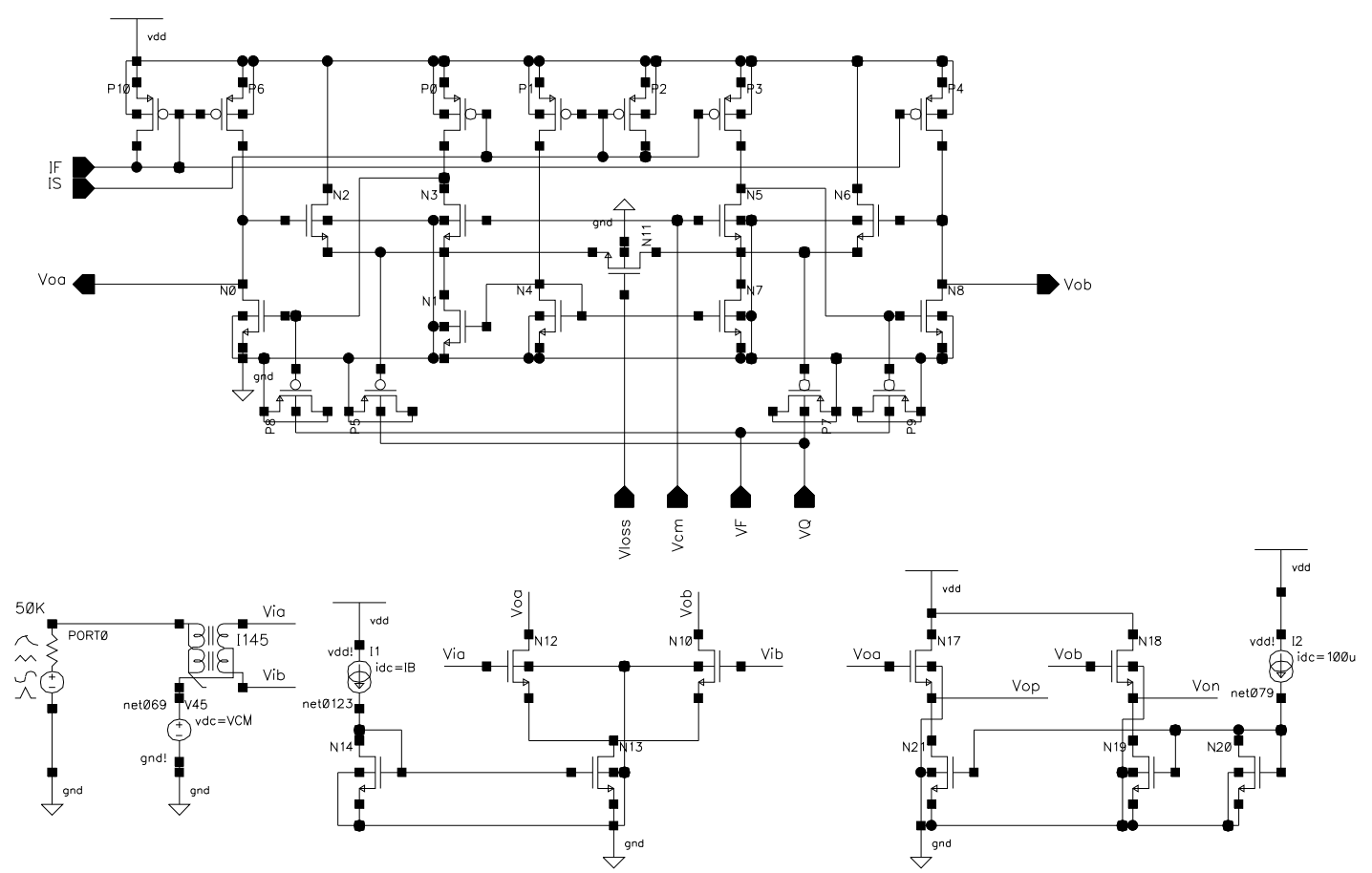

Figure 3-6 Active-inductor bandpass filter example. 


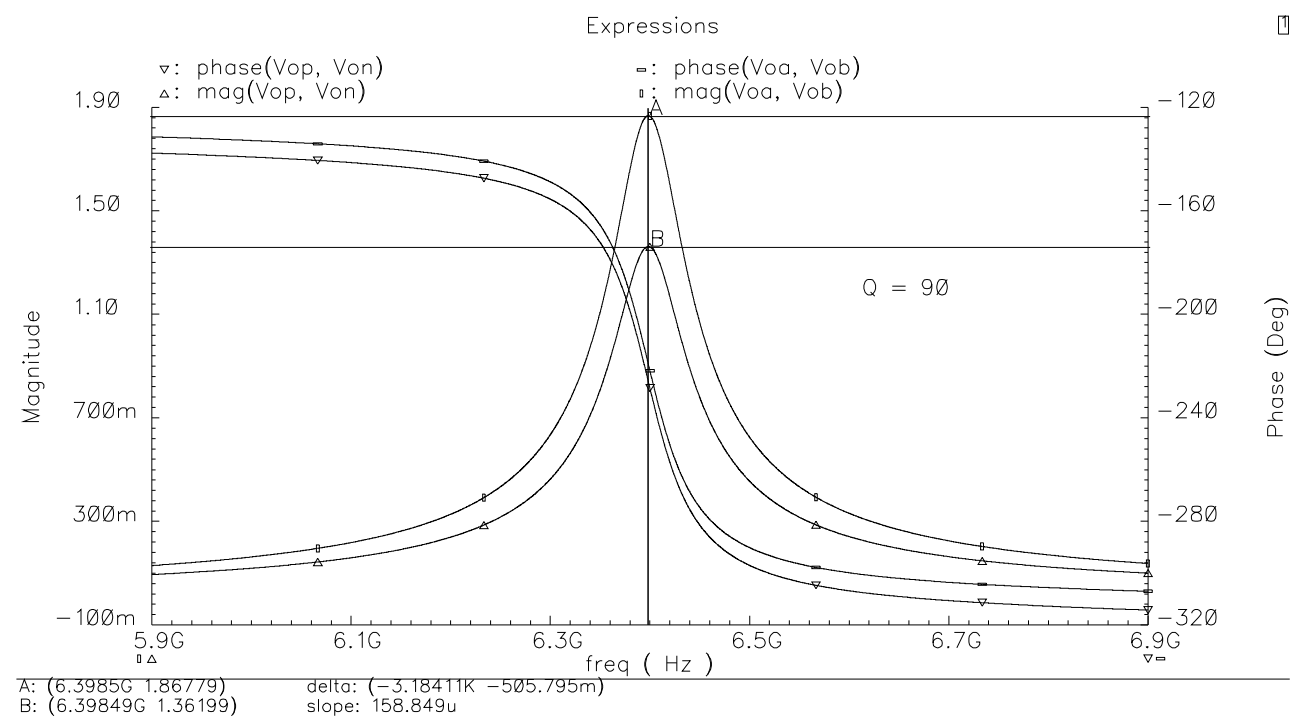

Figure 3-7 Transfer function from ac simulations (pseudo-differential).

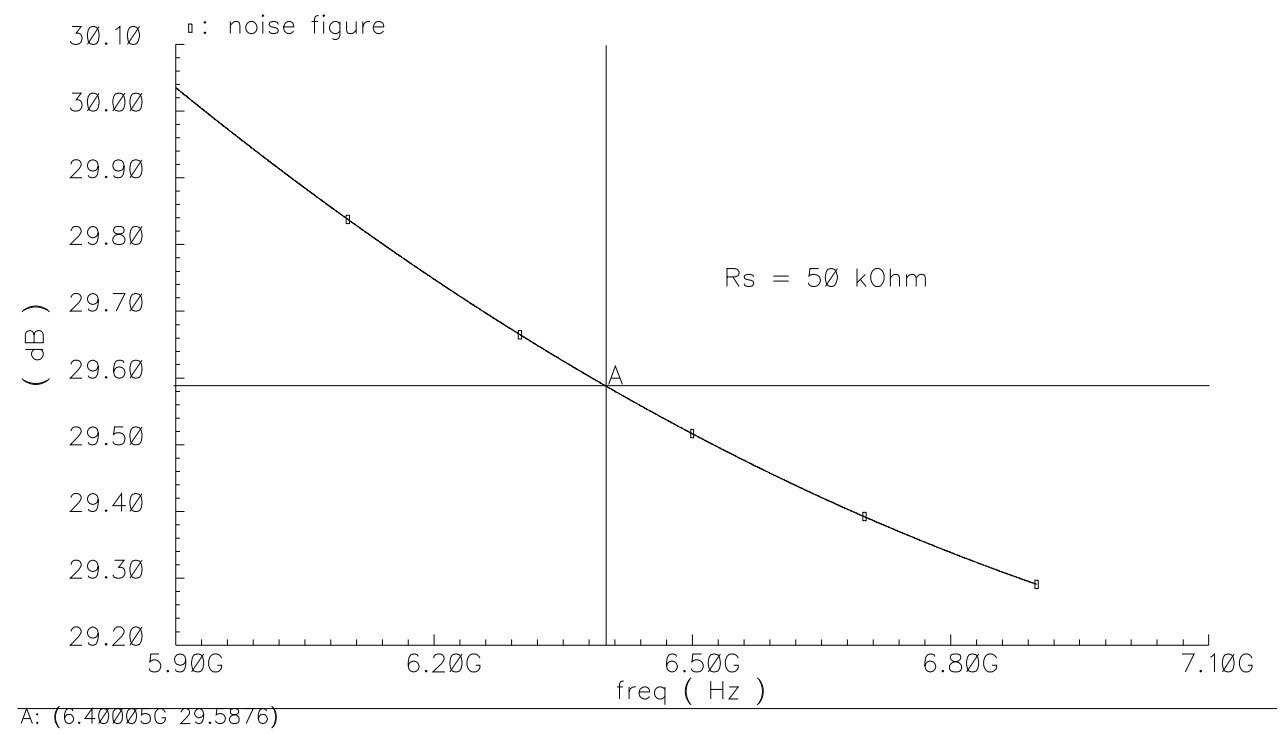

Figure 3-8 Noise simulation results, pseudo-differential, outputs taken at the source-follower output.

The small-signal parameters are found from the circuit's operating points: $g_{\mathrm{m} 1}=$ $1.2 \mathrm{mS}, g_{\mathrm{m} 2}=1.1 \mathrm{mS}, g_{\mathrm{m} 3}=1.2 \mathrm{mS}, C_{1}=21 \mathrm{fF}, C_{2}=18 \mathrm{fF}, C_{3}=19 \mathrm{fF}, g_{1}=132 \mu \mathrm{S}$, $g_{3}=112 \mu \mathrm{S}, G=g_{\mathrm{m} 1}+g_{\mathrm{m} 2}+g_{2}=2.44 \mathrm{mS}, G_{\mathrm{M}, \mathrm{i}}=17.25 \mu \mathrm{S}, G_{\mathrm{M}, \mathrm{o}}=481.2 \mu \mathrm{S}, G_{\mathrm{M}, \mathrm{o}, \text { load }}$ $=632.2 \mu \mathrm{S}$. The only parameters changed from the active inductor circuit are $g_{1}$ and $C_{1}$, due to the parasitic capacitance and leakage from the input $G_{\mathrm{M}}$ and output source 
follower. The circuit's performances are calculated using a spreadsheet using the equations in this work, as shown in Table 3-1. The calculated results match the simulation results very closely.

Table 3-1 Calculation of Active-Inductor Bandpass Performances

\begin{tabular}{|l|l|r|l|l|l|l|l|l|l|}
\hline $\begin{array}{l}\text { Para- } \\
\text { meter }\end{array}$ & Unit & Sims & $\begin{array}{l}\text { Calc- } \\
\text { 'ed }\end{array}$ & $\begin{array}{l}\text { Equ- } \\
\text { ation }\end{array}$ & $\begin{array}{l}\text { Para- } \\
\text { meter }\end{array}$ & Unit & Sims & $\begin{array}{l}\text { Calc- } \\
\text { 'ed }\end{array}$ & $\begin{array}{l}\text { Equ- } \\
\text { ation }\end{array}$ \\
\hline$g_{\mathrm{m} 1}$ & $\mathrm{mS}$ & 1.2 & & & GammaN & & 0.8786 & & $(2.73)$ \\
\hline$g_{\mathrm{m} 2}$ & $\mathrm{mS}$ & 1.1 & & & GammaP & & 0.9685 & & $(2.73)$ \\
\hline$g_{\mathrm{m} 3}$ & $\mathrm{mS}$ & 1.2 & & & KNF1 & & 3.6942 & & $(2.69)$ \\
\hline$C_{1}$ & $\mathrm{fF}$ & 21 & & & KNF2 & & 1.8471 & & $(2.71)$ \\
\hline$C_{2}$ & $\mathrm{fF}$ & 18 & & & & & & & \\
\hline$C_{3}$ & $\mathrm{fF}$ & 19 & & & $\mathrm{Rs}$ & $\mathrm{Mohm}$ & 0.05 & & \\
\hline$g_{1}$ & $\mathrm{uS}$ & 132 & & & & & & & \\
\hline$g_{3}$ & $\mathrm{uS}$ & 112 & & & & & & & \\
\hline$G$ & $\mathrm{mS}$ & 2.44 & & & & & & & \\
\hline$G_{\mathrm{M}, \mathrm{i}}$ & $\mathrm{uS}$ & 17.25 & & & & & & & \\
\hline$G_{\mathrm{M}, \mathrm{o}}$ & $\mathrm{uS}$ & 481.2 & & & & & & & \\
\hline$G_{\mathrm{M}, \mathrm{load}}$ & $\mathrm{uS}$ & 632.15 & & & & & & & \\
\hline & & & & & & & & & \\
\hline$f_{0}$ & $\mathrm{GHz}$ & $\mathbf{6 . 4}$ & $\mathbf{6 . 4 2 0}$ & $(3.10)$ & $\mathrm{NF}$ & & & 450.08 & $(3.17)$ \\
\hline$\omega_{0}$ & $\mathrm{Grad} / \mathrm{s}$ & & 40.34 & $(3.10)$ & $\mathrm{NF}$ & $\mathrm{dB}$ & $\mathbf{2 9 . 5 9}$ & 26.53 & $(3.17)$ \\
\hline$Q_{0}$ & & $\mathbf{9 0}$ & $\mathbf{8 9 . 4}$ & $(3.11)$ & $\mathrm{NF}+3 \mathrm{~dB}$ & $\mathrm{~dB}$ & & $\mathbf{2 9 . 5 4}$ & $(3.17)$ \\
\hline$H_{\mathrm{M}, \mathrm{V} 2}$ & & $\mathbf{1 . 8 7}$ & $\mathbf{1 . 8 2}$ & $(3.11)$ & & & & & \\
\hline$H_{\mathrm{M}, \mathrm{V} 3}$ & & $\mathbf{1 . 3 6}$ & $\mathbf{1 . 3 9}$ & $(3.11)$ & & & & & \\
\hline$R_{\mathrm{p}}$ & $\mathrm{MOhm}$ & & 0.106 & $(3.10)$ & & & & & \\
\hline
\end{tabular}

\subsection{The RF Lowpass Filters in this Work}

\subsubsection{All-NMOS-II Active Inductor Lowpass Filter}

The all-NMOS-II active inductor circuit, when configured as an analog signal driver, has the characteristics of a lowpass filter. Details were discussions in Section 2.8.3. The gate of the non-inverting transconductor in the all-NMOS-II circuit is freed from ac ground and used as a voltage input, and per Section 3.1.2, it will behave as a lowpass filter. Incidentally, due to the special circuit architecture, the lowpass filter has very high linearity. 


\subsubsection{All-NMOS Active Inductor Lowpass Filter}

The all-NMOS active inductor circuit can be readily converted into a lowpass filter by using the $V_{\mathrm{CM}}$ terminal on the $M_{2}$ gate as input and taking the output voltage on the drain of $M_{3}$, as shown in Fig. 3-9. It uses the principle shown in Fig. 3-2, by converting $V_{\text {in }}$ into a current into node $V_{\mathrm{L}}$ and using $V_{1}$ as output voltage. Similar to the bandpass filter, $V_{\text {out }}$ needs to be buffered, to prevent the load and interconnect capacitance and conductance from loading the active inductor core. The circuit has similar properties as the all-NMOS-II analog signal driver, such as $V_{\mathrm{IN}}=V_{\text {OUT }}$. However, since $M_{1}$ and $M_{2}$ have different voltage $V_{\mathrm{ds}}, V_{\text {out }}$ does not exactly follow $V_{\text {in }}$ in dynamic operation: as a result, the circuit's linearity is not as good, and $V_{\mathrm{IN}} \approx V_{\mathrm{OUT}}$ instead of being exactly equal.

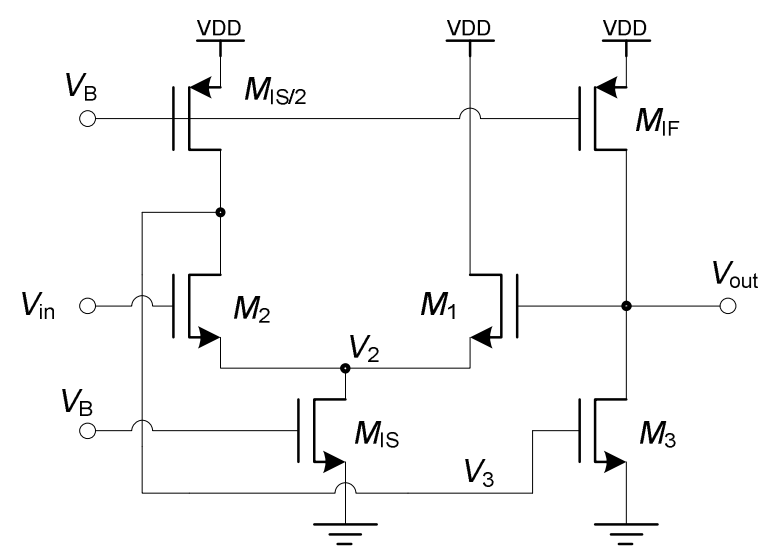

Figure 3-9 Implementing a lowpass filter using the all-NMOS active inductor. 


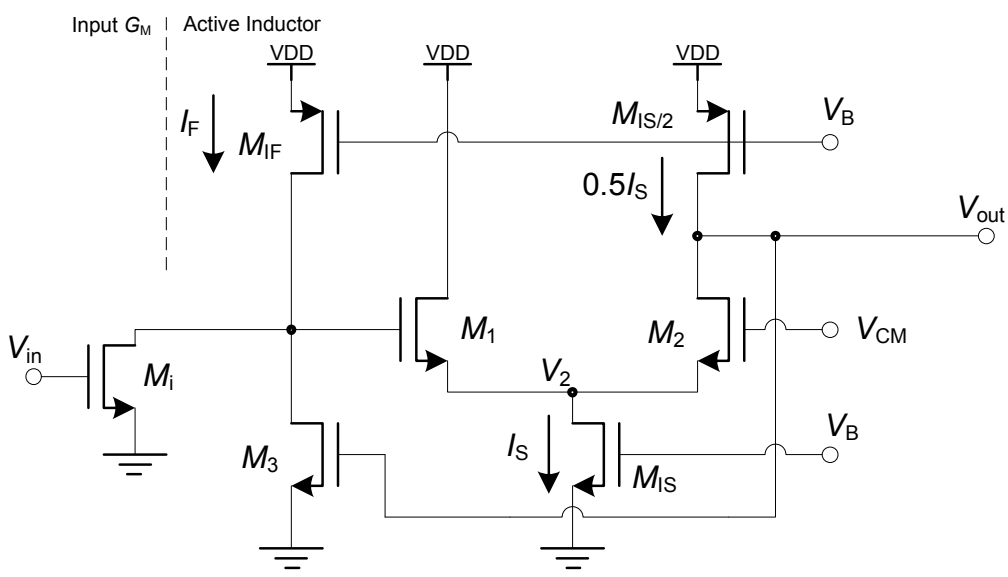

Figure 3-10 Another method of implementing a lowpass filter using the all-NMOS active inductor.

Another method of implementing a lowpass filter is shown in Fig. 3-10, where $M_{\mathrm{i}}$ converts $V_{\text {in }}$ into a current on node $V_{1}$, and $V_{\text {out }}$ is taken from the "load" node of the gyrator, as described in Fig. 3-2. This method allows the common-mode of $V_{\text {in }}$ and $V_{\text {out }}$ to be different, especially when $M_{\mathrm{i}}$ is implemented as a differential pair, at the cost of adding the $M_{\mathrm{i}}$ circuitry and slightly higher power dissipation and noise.

\subsection{Conclusion}

Bandpass and lowpass filters can be readily implemented with active inductors. To obtain high operating frequency, the filter function must be incorporated into the active-inductor circuit itself, rather than treating the active inductor as a pure inductor. For an arbitrarily given active-inductor circuit, by locating the two nodes of the gyrator, lowpass filters can be readily obtained using the principles given in this chapter.

The all-NMOS active-inductor bandpass filter is discussed in detail, and the performance equations are verified by simulation results. 


\section{CHAPTER 4}

\section{DESIGN OF RF ANALOG OSCILLATORS}

\subsection{Designing RF Oscillators with Active Inductors}

\subsubsection{A Passive $L-C$ Oscillator}

There are two types of oscillators operating at several $\mathrm{GHz}$ : ring oscillators and $L-C$ oscillators. A ring oscillator is a loop of an odd number of inverters, and each inverter can be a CMOS inverter or a resistor-loaded differential amplifier [84]. The inverter acts as both the gain- and amplitude-limiting element, thus its output voltage has high harmonic distortion and appears typically as rounded pulses. This is not an issue for digital circuitry since their clocks are pulses; however, for many other applications, such as modulation/demodulation, sinusoidal signals are required with low output harmonics and low phase noise, and this is almost always done with oscillators based on passive $L-C$ elements, labeled here simply as passive $L-C$ oscillators.

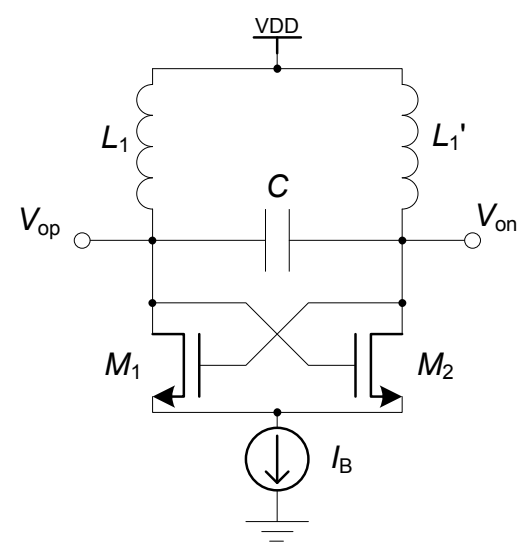

Figure 4-1 A passive $L-C$ oscillator.

Figure 4-1 shows a typical passive $L-C$ oscillator for operations between 1-10 GHz. The passive inductors $L_{1}$ and $L_{1}{ }^{\prime}$ are symmetrical and contained within one single spiral inductor. $C$ is usually a pair of varactors connected back to back to 
facilitate frequency tuning. To obtain a certain frequency tuning range, $C$ has to vary between $C_{\max }$ and $C_{\min }$ and cannot be zero, and this limits the oscillation frequency from approaching the self-resonance frequency of the inductors. $M_{1}, M_{2}$ and $I_{\mathrm{B}}$ implement a negative resistor, providing the gain for the oscillation to start and continue. $M_{1}$ and $M_{2}$ also limit the oscillation amplitude. The output voltage (power) is available through the $V_{\text {op }}$ and $V_{\text {on }}$ terminals. The oscillator can only be differential due to the cross connection of $M_{1}$ and $M_{2}$, and this happens to be desirable and not an issue for most applications. Depending on the particular design, the oscillation may be current- or voltage-limited:

1. Current limited. The voltage amplitudes on $V_{\text {op }}$ and $V_{\text {on }}$ can fully turn on and off $M_{1}$ and $M_{2}$ and make them operating in switching mode, but is smaller than $V_{\mathrm{DD}}$. The biasing current $I_{\mathrm{B}}$ is alternated between $L_{1}$ and $L_{1}{ }^{\prime} . I_{\mathrm{B}}$ and the "tank" loss determine the voltage amplitude.

2. Voltage limited. The voltage amplitudes on $V_{\text {op }}$ and $V_{\text {on }}$ are so high that they are reaching $\sim 2 \cdot V_{\mathrm{DD}}$ at their high points and turning off $M_{1}$ and $M_{2}$ at low points.

\subsubsection{An Active-Inductor Oscillator}

Active inductors can be used to replace the passive inductors in Fig. 4-1 to implement oscillators. The varactor $C$ is not needed, since the active inductor is tunable, and its $f_{\mathrm{R}}$ determines the oscillation frequency $f_{0}$. The circuit is shown in Fig. 4-2 where each active inductor is represented by its equivalent circuit. Since the active-inductor circuit can be designed differentially or pseudo-differentially, it naturally provides a dual $R$ $L-C$ tank for the differential structure. The output voltages are obtained from the 
inductor port, and just like the active-inductor filters, a voltage buffer, e.g., a source follower, is needed to prevent the load from reducing $f_{\mathrm{R}}$ and $Q$ of the tank.

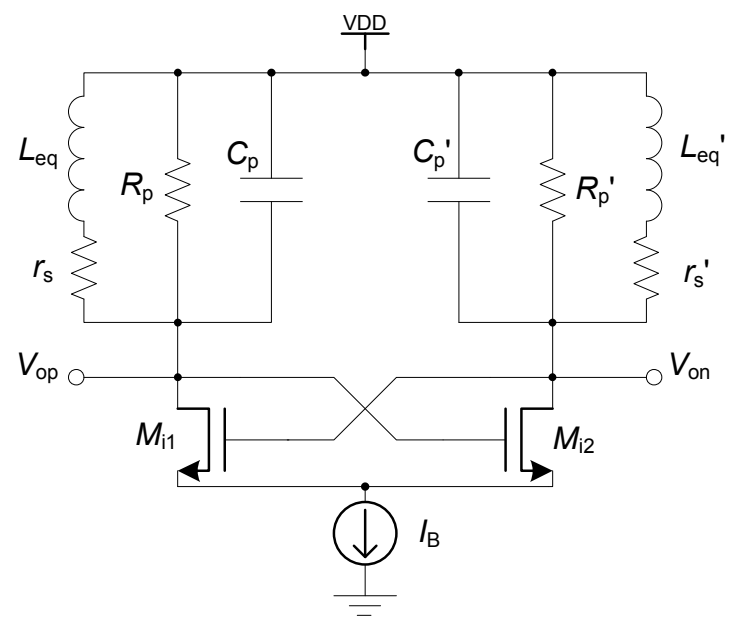

Figure 4-2 An active-inductor oscillator.

The equivalent active-inductor components in Fig. 4-2 are calculated with the extra parasitic capacitances and conductances of $M_{\mathrm{i} 1}$ and $M_{\mathrm{i} 2}$ as well as of the output buffers (not shown in figure) absorbed.

\subsubsection{Reducing Output Voltage Harmonic Distortion}

Equating $M_{1}$ and $M_{2}$ in Fig. 4-2 circuit to a negative resistor is convenient and is the common microwave-design method $[85,86]$. However, different from the microwave design, where the negative resistor is almost always the amplitude limiting element, since the impedance-transformation network to which it is connected is usually passive and has much higher linearity, amplitude limiting in active-inductor oscillators can happen anywhere, since the whole circuit is active.

This active-inductor oscillator operates in voltage mode, and we need the differential output voltage, $V_{\text {op }}-V_{\text {on }}$, to be low in harmonic distortion. However, 
different from a passive $L-C$ oscillator, the active inductor is nonlinear and has a low limit on its maximum signal amplitude, since it is made of transistors. Thus, besides the negative resistor $M_{1}-M_{2}$, the active inductor can also limit the oscillation amplitude. Further design analysis is needed.

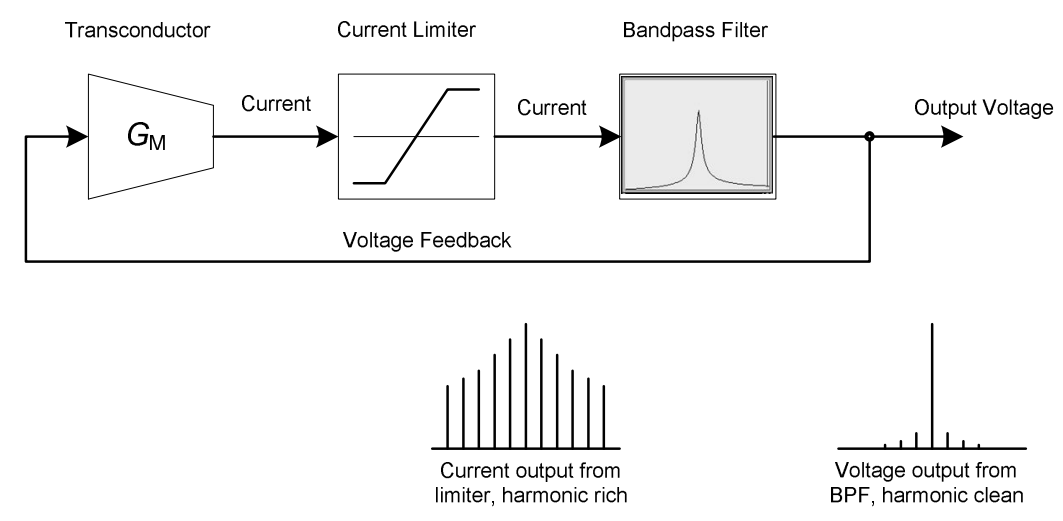

Figure 4-3 Active-inductor oscillator block diagram.

Figure 4-3 shows the block diagram of the active-inductor oscillator. The signals are drawn as single lines for simplicity, and are differential in a real circuit implementation. The $G_{\mathrm{M}}$ (the $M_{1}-M_{2}$ differential pair) converts the output voltage into a current. The bandpass filter is in fact the active inductor by itself, and has current input and voltage output, that is $T(s)=Z_{\text {in }}(s)$. In between is a current limiter, whose output is clamped and rich in harmonics due to its limiting effect. The bandpass filter eliminates most of the harmonics in the input current and outputs a harmonically clean voltage. The current limiter, as we shall see in more detail, is actually part of the function of the differential pair $M_{1}-M_{2}$. Qualitatively, the minimum and maximum output currents from $M_{1}$ or $M_{2}$ drains are 0 and $I_{\mathrm{B}}$, respectively, therefore $M_{1}$ and $M_{2}$ implement the $G_{\mathrm{M}}$ and the current limiter at the same time.

To ensure the above scenario happens as planned, $I_{\mathrm{B}}$ must be kept at a low level, 
such that at the output current peak at $G_{\mathrm{M}}$, the active-inductor BPF output voltage is not clamped or distorted. If $I_{\mathrm{B}}$ is high, the active inductor will become the limiting element in the feedback loop, and its output voltage will be distorted. It should be noted that due to the high operating frequency and effects from parasitic capacitances, we will not see clipped current or voltage signals; instead, they will be compressed at their peaks when limited.

The transfer function of the bandpass filter has the impedance profile of the active inductor. For the circuit in Fig. 4-2, neglecting $r_{\mathrm{s}}$ since at $f \approx f_{\mathrm{R}}\left|R_{\mathrm{p}}\right|>>r_{\mathrm{s}}$, we have

$$
T(s)=Z_{\text {in }}(s)=\frac{s / C_{\mathrm{p}}}{s^{2}+s /\left(R_{\mathrm{p}} C_{\mathrm{p}}\right)+1 /\left(L_{1} C_{\mathrm{p}}\right)}=\frac{s \frac{\omega_{\mathrm{R}}}{Q_{\mathrm{R}}} R_{\mathrm{p}}}{s^{2}+s \frac{\omega_{\mathrm{R}}}{Q_{\mathrm{R}}}+\omega_{\mathrm{R}}^{2}}
$$

with the magnitude being

$$
|T(\omega)|=\frac{\omega \frac{\omega_{\mathrm{R}}}{Q_{\mathrm{R}}} R_{\mathrm{p}}}{\sqrt{\left(\omega_{\mathrm{R}}^{2}-\omega^{2}\right)^{2}+\left(\omega \frac{\omega_{\mathrm{R}}}{Q_{\mathrm{R}}}\right)^{2}}}
$$

Thus we have

$$
\left|T\left(\omega_{\mathrm{R}}\right)\right|=R_{\mathrm{p}}, \quad\left|T\left(2 \omega_{\mathrm{R}}\right)\right|=\frac{R_{\mathrm{p}}}{\sqrt{\frac{9}{4} Q_{\mathrm{R}}^{2}+1}}, \quad\left|T\left(3 \omega_{\mathrm{R}}\right)\right|=\frac{R_{\mathrm{p}}}{\sqrt{\frac{64}{9} Q_{\mathrm{R}}^{2}+1}}
$$

The higher $Q_{\mathrm{R}}$ is, the higher is the attenuation of the harmonic components in the input current, with a maximum limit on $Q_{\mathrm{R}}$ as we will see in the next section. The phase of this bandpass filter is

$$
\angle T(s)=\frac{\pi}{2}-\tan ^{-1} \frac{\omega \omega_{\mathrm{R}} / Q_{\mathrm{R}}}{\omega_{\mathrm{R}}^{2}-\omega^{2}}=0 \text {, when } \omega=\omega_{\mathrm{R}}
$$




\subsubsection{Designing the Oscillator Loop Gain}

Common perception has it that since the oscillator in Fig. 4-3 has a high- $Q$ activeinductor BPF as "frequency-selecting element," the oscillation frequency $f_{0}$ will automatically be the BPF center frequency, $f_{\mathrm{R}}$, because the BPF "selects" the oscillation frequency. In reality, $f_{0}$ usually does not equal $f_{\mathrm{R}}$, due to the phase shifts from the parasitic elements inside and outside of the devices. There are two necessary conditions to build up and sustain a stable oscillation:

1. The loop gain is greater than 1 , that is, $G_{\mathrm{M}}>1 / R_{\mathrm{p}}$, with margin, as we will see below.

2. The loop phase shift is a multiple of $2 \pi$.

The final steady-state frequency is determined by the phase condition, and not because the frequency-selecting element "selected" the frequency. Passive- and activeinductor resonators come in handy for designing oscillators, because when placed in the feedback loop, as in Fig. 4-3, they happen to have the maximum impedance (and in turn, gain) at $f_{\mathrm{R}}$, when their phase is $0^{\circ}$. The rejection of frequencies away from $f_{\mathrm{R}}$ is hence a desirable coincidence. For active inductors, the maximum impedance, $R_{\mathrm{p}}$, is approximately proportional to $Q_{\mathrm{R}}$ (Eq. 2.8). Therefore, the higher $Q_{\mathrm{R}}$ is, the better does the resonator filter out unwanted harmonics per Eq. (4.3). However, for GHzoscillator design, besides the high circuit sensitivities associated with high $Q_{\mathrm{R}}$, there is another issue.

Figure 4-4 shows the magnitude and phase profile of an example resonator, with a very much scaled down $f_{\mathrm{R}}$. At $f_{\mathrm{R}}=3 \mathrm{kHz}$, its phase $\phi=0$ and $R_{\mathrm{P}}=100 \Omega$. When 
placed in the loop in Fig. 4-3, if $G_{\mathrm{M}}$ and current limiter both have $0^{\circ}$ phase shift, we expect the oscillation frequency $f_{0}=f_{\mathrm{R}}=3 \mathrm{kHz}$, and to make the loop gain $>1$ we need $G_{\mathrm{M}}>10 \mathrm{mS}$. However, if due to parasitic capacitance, $G_{\mathrm{M}}$, current limiter and signal wires have a total phase shift of, say, $-35^{\circ}$, to satisfy the $0^{\circ}$-loop-phase requirement, $f_{0}$ will shift down to approximately $2.85 \mathrm{kHz}$, and the magnitude is reduced to $83 \Omega$. To oscillate, $G_{\mathrm{M}}$ will have to be greater than $12 \mathrm{mS}$. If $G_{\mathrm{M}}$ is not designed with sufficient margin, and/or $Q_{\mathrm{R}}$ is overly high, the oscillator may not oscillate. When $f_{0} \neq f_{\mathrm{R}}$, the resonator will amplify the desired signal less and the circuit noise more, another unwanted result.

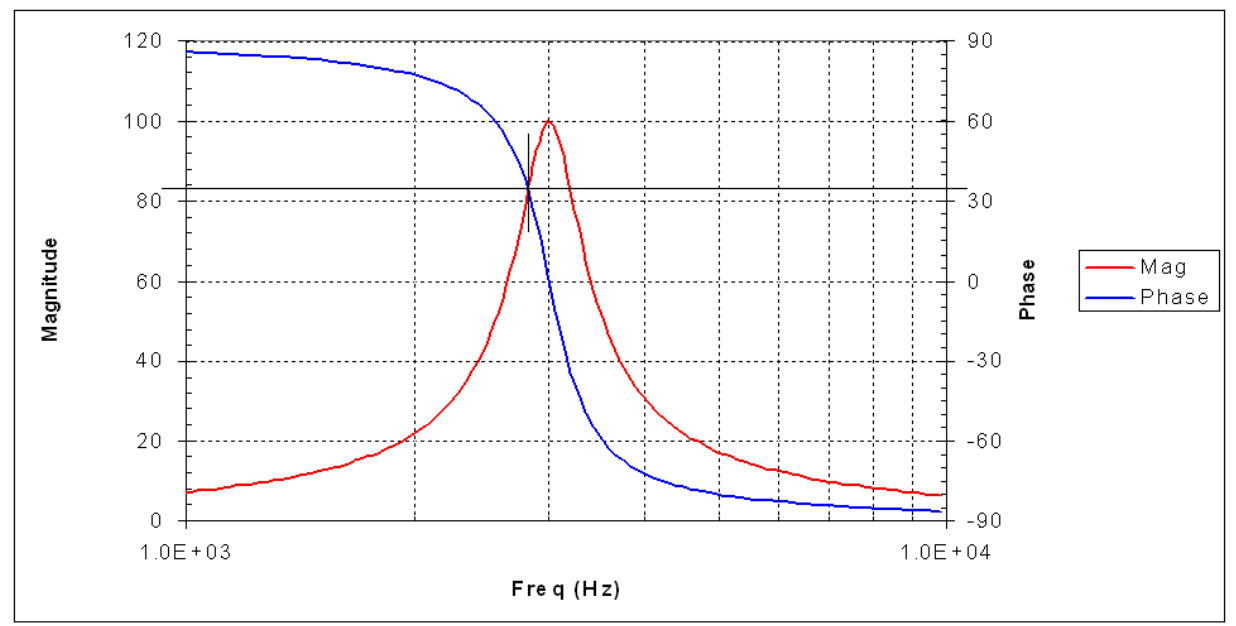

Figure 4-4 The magnitude and phase of an example shunt $R-L-C$ resonator.

This phenomenon is highly visible in $\mathrm{GHz}$ designs because of the high frequency. To generate $35^{\circ}$ phase lag at $3 \mathrm{kHz}$ and $3 \mathrm{GHz}$, delays of $32.4 \mu$ s and 32.4 ps, respectively, are needed. Therefore, the distributed- $R$ - $C$ or transmission-line delays in IC interconnections will be much more sensitive in the $3 \mathrm{GHz}$ design than in the 3 $\mathrm{kHz}$ design. In addition, if $G_{\mathrm{M}}$ has an internal pole, its phase will increase with frequency. 


\subsection{RF Oscillators in This Work}

\subsubsection{The Circuit and AC Analysis}

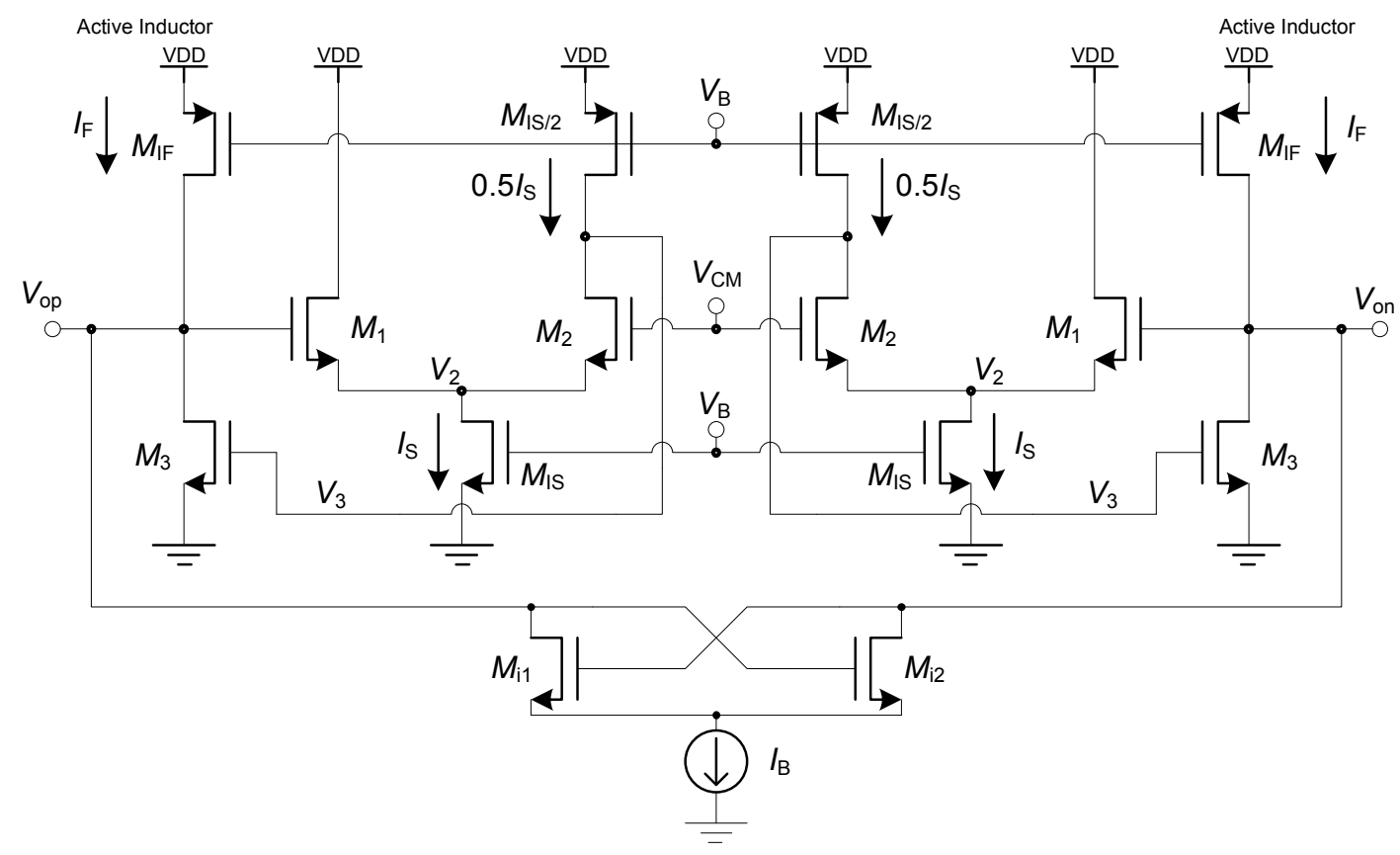

Figure 4-5 The all-NMOS active-inductor oscillator in a pseudo-differential structure.

Figure 4-5 shows the all-NMOS active-inductor oscillator in a pseudo-differential structure. The differential pair $M_{\mathrm{i} 1}-M_{\mathrm{i} 2}$ implements both the gain element and the current limiter in the feedback loop. The required source followers at $V_{\text {op }}$ and $V_{\text {on }}$ are not shown for clarity. $M_{\mathrm{i} 1}$ and $M_{\mathrm{i} 2}$ can be assumed to have very low phase shift since their parasitic capacitance can be considered as extrinsic. When $Q_{\mathrm{R}}$ of the active inductor is sufficiently high $(>\sim 20)$, the oscillation frequency of the circuit is approximately the active inductor self resonance frequency $f_{\mathrm{R}}$ (Eq. 2.55),

$$
\omega_{0} \approx \omega_{\mathrm{R}}=\sqrt{\frac{g_{\mathrm{m} 1} g_{\mathrm{m} 2} g_{\mathrm{m} 3}}{G C_{1} C_{3}}} \approx \sqrt{\frac{0.5 g_{\mathrm{m} 1} g_{\mathrm{m} 3}}{C_{1} C_{3}}}=\frac{1}{\sqrt{L_{\mathrm{EQ}} C_{1}}}
$$

The active-inductor impedance at $f_{\mathrm{R}}$ is (Eq. 2.62), 


$$
R_{\mathrm{p}}=\frac{1}{g_{1}+\frac{C_{1}}{C_{3}} g_{3}+\frac{C_{2} g_{1} g_{3}}{C_{3} G}-\omega^{2} \frac{C_{1} C_{2}}{G}}=\frac{Q_{\mathrm{R}}}{\omega_{\mathrm{R}} C_{1}}=Q_{\mathrm{R}} \sqrt{\frac{L_{\mathrm{eq}}}{C_{1}}}
$$

When $M_{\mathrm{i} 1}$ and $M_{\mathrm{i} 2}$ operate as current limiter as discussed before, the single-ended and differential output-signal amplitudes are approximately

$$
\begin{aligned}
& V_{\mathrm{o}, \mathrm{a}} \approx \frac{I_{\mathrm{B}} \cdot R_{\mathrm{p}}}{2}=\frac{I_{\mathrm{B}} / 2}{g_{1}+\frac{C_{1}}{C_{3}} g_{3}+\frac{C_{2} g_{1} g_{3}}{C_{3} G}-\omega^{2} \frac{C_{1} C_{2}}{G}}=\frac{I_{\mathrm{B}} Q_{\mathrm{R}}}{2 \omega_{\mathrm{R}} C_{1}}=\frac{I_{\mathrm{B}} Q_{\mathrm{R}}}{2} \sqrt{\frac{L_{\mathrm{eq}}}{C_{1}}} \text {, single-ended } \\
& V_{\mathrm{o}, \mathrm{d}}=2 \cdot V_{\mathrm{o}, \mathrm{a}}, \text { differentially. }
\end{aligned}
$$

The factor of 2 in the denominator of $V_{\mathrm{oa}}$ is because $2 V_{\mathrm{o}, \mathrm{a}}=I_{\mathrm{B}} R_{\mathrm{p}}-0 R_{\mathrm{p}}$.

The circuit parameters used above need to be updated to reflect the extra parasitic capacitance and conductance from the differential pair and output drivers:

$$
\begin{aligned}
& C_{1}=C_{1, \text { active inductor }}+4 \cdot C_{\mathrm{gd}, \mathrm{Mi}}+C_{\mathrm{jd}, \mathrm{Mi}}+C_{\mathrm{gs}, \mathrm{Mi}} \\
& g_{1}=g_{1, \text { active inductor }}+g_{\mathrm{ds}, \mathrm{Mi}}
\end{aligned}
$$

\subsubsection{Oscillator-Noise Analysis}

Since oscillators, whether built with passive or active inductors, must have active elements to replenish energy, they inevitably generate noise (a passive inductor generates noise, too, but at a lower level compared with active devices). The output noise of oscillators can be projected on two dimensions, amplitude noise and phase noise. Amplitude noise represents the random signal amplitude variation and is usually less of a concern since all oscillators have amplitude-regulating mechanism, and the noise contribution from active elements to the oscillation amplitude is largely dissipated before reaching the output. Phase noise represents the short-term periodic 
fluctuation of the oscillation, and regularly gains designers' attention for a couple of reasons:

1. It is detrimental since it can introduce adjacent channel interference in communication systems $[83,87]$ and it cannot be easily reduced.

2. It is not thoroughly modeled and unanimously understood, even though simulators have progressed in predicting the phase noise in recent years.

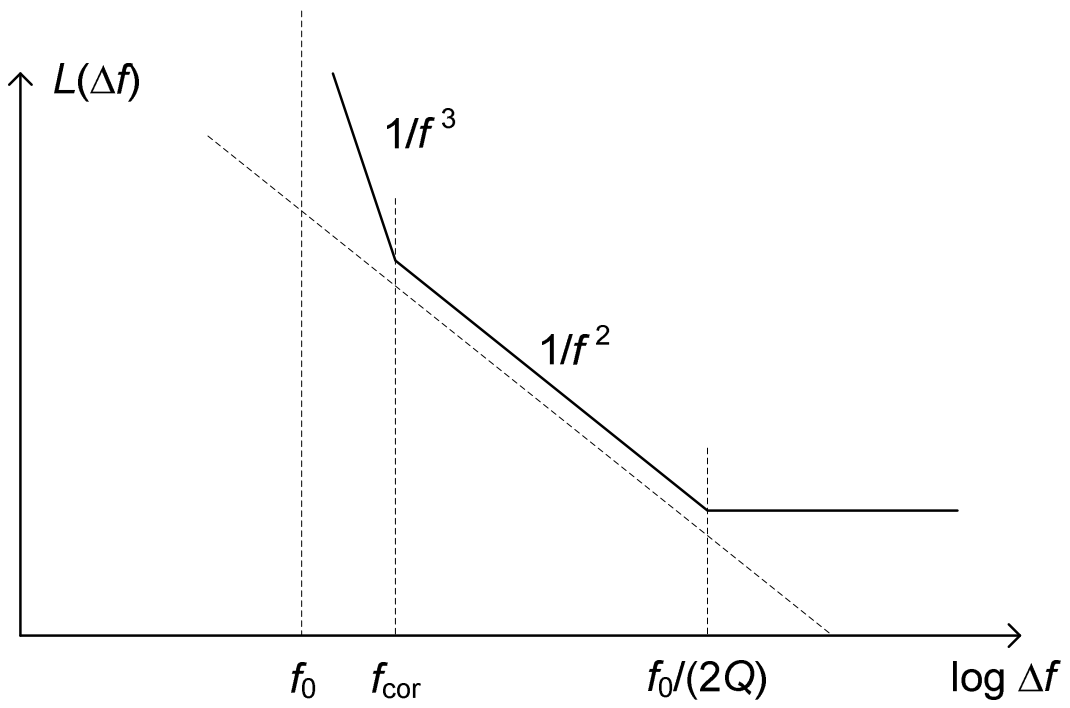

Figure 4-6 A typical oscillator phase-noise profile.

A typical phase-noise profile is shown in Fig. 4-6, where $L(\Delta f)$ represents the squared phase-noise voltage or current, or power. At frequencies close to the oscillation frequency $f_{0}, L(\Delta f)$ is proportional to $1 / f^{\beta}$ due to flicker noise; at intermediate frequencies, $L(\Delta f)$ is proportional to $1 / f^{2}$ due to thermal noise; and beyond a certain frequency, phase noise reaches a floor and does not reduce any further. Phase noise does not become infinite when $\Delta f$ approaches 0 , but shows a Lorentzian spectrum profile at or near $f_{0}[83]$.

Leeson introduced a phenomenological model of phase noise in 1966 [88] 
modeling the $1 / f^{2}$ and the flat portions of phase noise and has been generously referred in the study of phase noise in recent years. Leeson's model, however, contains a few empirical parameters that are circuit- and process-dependent and must be determined experimentally or empirically. In the past decade or so, Razavi, Lee and Abidi contributed independent theories that treat the phase noise from different approaches $[1,83,87,89,90]$.

There are two steps in providing an analytical expression of oscillator phase noise: (1) how is the thermal noise converted into phase noise; (2) up-conversion of the flicker $(1 / f)$ noise.

Lee solved the thermal noise conversion by examining the impedance profile of an inductor resonator with loss compensation [83], which is infinite at the oscillator frequency $f_{0}$ in steady state, since the compensation must cancel the circuit loss perfectly, no more, no less. The thermal noise is modeled as a shunt white noise current, which is shaped by the impedance profile of the resonator. Razavi approaches this issue by studying the closed-loop transfer-function sensitivity to noise, and shows the identical result, with the added benefit of being able to apply the theory on nonresonator oscillators, such as ring oscillators.

The flicker noise of active devices near $\mathrm{dc}$ is up-converted into $1 / f^{3}$ phase noise near $f_{0}$ due to circuit nonlinearities. Lee solved this problem by introducing periodic time-varying impulse sensitivity functions; Razavi's method is similar, but done in the frequency domain. The flicker noise conversion is important for low-noise oscillators, such as passive-inductor oscillators. The phase-noise level of active-inductor oscillators is dominated by thermal noise which is $30-40 \mathrm{~dB}$ higher than that of 
passive inductors at practical power levels [90], therefore the $1 / f^{3}$ region is hardly visible. Thus we concentrate on the $1 / f^{2}$ phase noise, or thermal noise conversion only.

The phase noise floor is usually empirically modeled and not analyzed by the researchers. It is not modeled in circuit simulators either. Therefore, we will not try to analyze it.

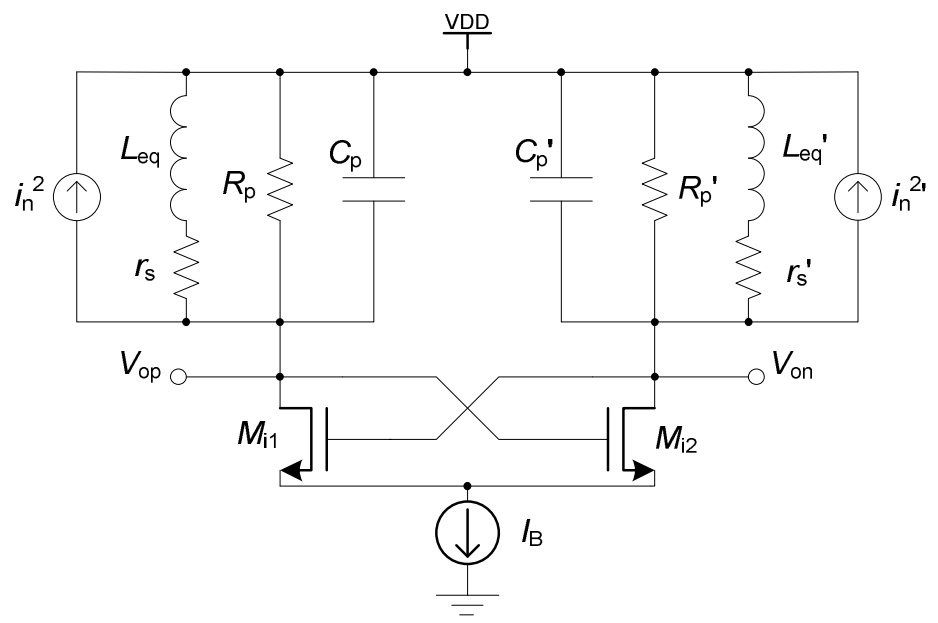

Figure 4-7 The equivalent circuit of the active-inductor oscillator showing the noise elements.

Figure 4-7 shows the equivalent circuit of the active-inductor oscillator with noise elements. Per discussions in Chapter 2, the shunt noise current $i_{\mathrm{n}}{ }^{2}$ sufficiently represents the active-inductor noise and is given by Eq. (2.50). For the oscillator, $i_{\mathrm{n}}{ }^{2}$ must also include the noise from $M_{\mathrm{i} 1}$ and $M_{\mathrm{i} 2}$,

$$
\begin{aligned}
& i_{\mathrm{n}}^{2}=4 k T\left(K_{\mathrm{NF} 2} G_{\mathrm{M} 2}+\frac{\omega_{\mathrm{R}}^{2}}{\omega^{2}} K_{\mathrm{NF} 1} G_{\mathrm{M} 1}+\Gamma_{\mathrm{n}} G_{\mathrm{Mi1}}\right) \\
& \approx 4 k T\left(K_{\mathrm{NF} 2} G_{\mathrm{M} 2}+K_{\mathrm{NF} 1} G_{\mathrm{M} 1}+\Gamma_{\mathrm{n}} G_{\mathrm{Mi1}}\right)
\end{aligned}
$$

when $\omega \approx \omega_{\mathrm{R}} . K_{\mathrm{NF} 1}$ and $K_{\mathrm{NF2}}$ for the all-NMOS active inductor are given by Eqs. (2.69) and (2.71), respectively. The noise from the tail current source $I_{\mathrm{B}}$ generates mostly a common-mode voltage and is neglected.

The loss of the active-inductor resonator at steady oscillation is $100 \%$ 
compensated by the negative resistor, no more, no less, and as a result, the impedance looking into the oscillator output is $\infty$, just like in a lossless $L-C$ resonator:

$$
Z(\omega)=\frac{1}{j \omega C_{1}+\frac{1}{j \omega L_{\mathrm{eq}}}}=\frac{j \omega L_{\mathrm{eq}}}{1-\frac{\omega^{2}}{\omega_{0}^{2}}}=\frac{j\left(\omega_{0}+\Delta \omega\right) L_{\mathrm{eq}}}{1-\frac{\left(\omega_{0}+\Delta \omega\right)^{2}}{\omega_{0}^{2}}}=\frac{j\left(\omega_{0}+\Delta \omega\right) L_{\mathrm{eq}}}{1-1-2 \frac{\Delta \omega}{\omega_{0}}-\frac{\Delta \omega^{2}}{\omega_{0}^{2}}} \approx-\frac{j \omega_{0} L_{\mathrm{eq}}}{2 \frac{\Delta \omega}{\omega_{0}}}
$$

Using the relationship of $\omega_{0}^{2}=1 /\left(L_{\mathrm{eq}} C_{1}\right)$, it becomes

$$
Z(\omega)=-j \frac{\omega_{0} L_{\mathrm{eq}}}{2 \frac{\Delta \omega}{\omega_{0}}}=-j \frac{1}{2 \Delta \omega C_{1}}
$$

The noise voltage at the oscillator output therefore is

$$
\begin{aligned}
& \frac{v_{\mathrm{n}}^{2}}{\Delta f}=\frac{i_{\mathrm{n}}^{2}}{\Delta f}|Z(\omega)|^{2}=\frac{i_{\mathrm{n}}^{2}}{\Delta f} \frac{1}{\left(2 \Delta \omega C_{1}\right)^{2}} \\
& \approx 4 k T\left(K_{\mathrm{NF} 2} G_{\mathrm{M} 2}+K_{\mathrm{NF} 1} G_{\mathrm{M} 1}+\Gamma_{\mathrm{n}} G_{\mathrm{Mi} 1}\right) \frac{1}{\left(2 \Delta \omega C_{1}\right)^{2}} \\
& =\frac{k T\left(K_{\mathrm{NF} 2} G_{\mathrm{M} 2}+K_{\mathrm{NF} 1} G_{\mathrm{M} 1}+\Gamma_{\mathrm{n}} G_{\mathrm{Mi} 1}\right)}{\left(\Delta \omega C_{1}\right)^{2}}
\end{aligned}
$$

Phase noise is the ratio of noise voltage to the signal amplitude. Using Eq. (4.7), we have,

$$
\begin{aligned}
& L(\omega)=10 \log \left(\frac{\frac{v_{\mathrm{n}}^{2}}{\Delta f}}{v_{\mathrm{a}}^{2} / 2}\right) \\
& =10 \log \frac{8 \omega_{0}^{2} k T\left(K_{\mathrm{NF} 2} G_{\mathrm{M} 2}+K_{\mathrm{NF} 1} G_{\mathrm{M} 1}+\Gamma_{\mathrm{n}} G_{\mathrm{Mi} 1}\right)}{\Delta \omega^{2} I_{\mathrm{B}}^{2} Q_{\mathrm{R}}^{2}}, \mathrm{dBc} / \mathrm{Hz}
\end{aligned}
$$

The division by two in the denominator, $V_{\mathrm{a}}^{2} / 2$, is because $V_{\mathrm{a}}$ is a peak value and needs to be converted into RMS, since noise is an RMS value.

In deriving the phase noise (Eq. 4.13), we use half of the pseudo-differential circuit to calculate both the noise and signal, therefore, unlike the bandpass filter (end 
of Section 3.2.2), we do not need to add $3 \mathrm{~dB}$ to the phase noise for a pseudodifferential circuit.

Lee [83] states that the thermal noise in Eq. (4.12) contributes equally to the fluctuations of oscillation amplitude and phase, i.e., AM noise and PM noise, per the equipartition theorem of thermodynamics; further, when an amplitude-limiting scheme exists, as in every practical oscillator, only half of the noise (PM noise) shows up as phase noise, and the other half (AM noise) disappears (presumably into heat). On the other hand, Ken Kundert, the primary designer of the Cadence ${ }^{\circledR}$ SpectreRF® simulator which we use to simulate the phase noise, states that when an amplitudelimiting scheme exists, the AM noise actually mostly turns into PM noise rather than disappearing; consequently, almost $100 \%$ of the noise in Eq. (4.12) shows up as phase noise [91]. The simulator is implemented assuming that the thermal noise is $100 \%$ converted into phase noise. The difference between Lee's theory and that of Kundert is $3 \mathrm{~dB}$. As shown by Eq. (4.13), we use Kundert's theory here, since this is how the simulator is implemented, and several RF engineers whom the author surveyed stated that they did not see a (3-dB) phase noise discrepancy between silicon and SpectreRF®.

Once again, Eq. (4.13) does not take into account the noise-to-frequency translation due to the oscillator nonlinearity and the phase noise floor. However, as we shall see below, it yields results with reasonable accuracy, mainly because the thermal noise of active-inductor oscillators is $30-40 \mathrm{~dB}$ higher than that of passive $L-C$ oscillators and it dominates all other sources and mechanism in overall phase noise. 


\subsubsection{Example of Active-Inductor Oscillator}

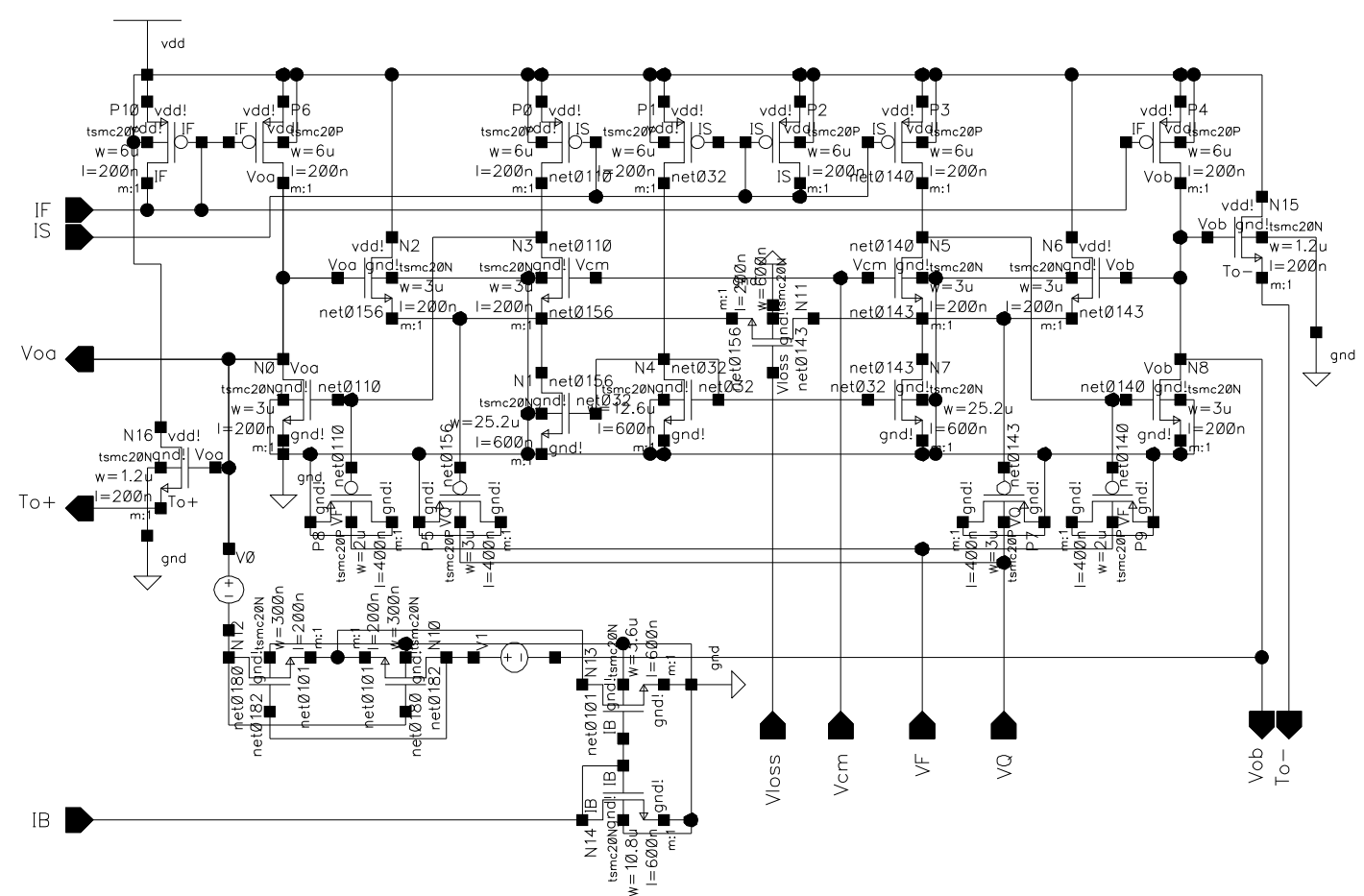

Figure 4-8 The oscillator circuit. $I_{\mathrm{S}}=I_{\mathrm{F}}=250 \mu \mathrm{A}, I_{\mathrm{B}}=50 \mathrm{uA}, V_{\mathrm{F}}=V_{\mathrm{Q}}=0.5, V_{\mathrm{CM}}=1.35, V_{\text {loss }}=0$.

The oscillator circuit in this design is shown in Fig. 4-8. $I_{\mathrm{F}}$ and $I_{\mathrm{S}}$ are external biasing currents that provide the biasing voltages for the single-transistor current sources P4, P6, P0, P3 and P1. The current from P1 is duplicated by NFETs N4, N1 and N7 to provide the tail currents for the differential pairs N2-N3 and N5-N6. PFETs P8-P9 and P5-P7 implement the varactors for frequency and $Q$ tuning, respectively. NFETs N0, $\mathrm{N} 2$ and N3 form an all-NMOS active inductor, and N5, N6, N8 form the mirrored active inductor to realize a pseudo-differential circuit.

$\mathrm{N} 11$, whose gate voltage is controlled by $V_{\text {loss }}$, operates in the linear region since its source and drain are connected to equal voltages. By increasing $V_{\text {loss, }}$ N11 can be turned on and acts as a resistor $R_{\text {loss }}$ across the differential circuit. $R_{\text {loss }}$ is equivalent to a grounded resistor of value $R_{\text {loss }} / 2$ for each half of the circuit. Since $R_{\text {loss }} / 2$ is in 
parallel with $g_{2}$ in Fig. 2-28, per Eq. (2.58), it has the effect of decreasing the circuit $Q_{\mathrm{R}}$. N11 is designed as a risk mitigation measure in case the circuit is unstable due to inaccurate transistor modeling or parasitic extractions and is normally turned off.

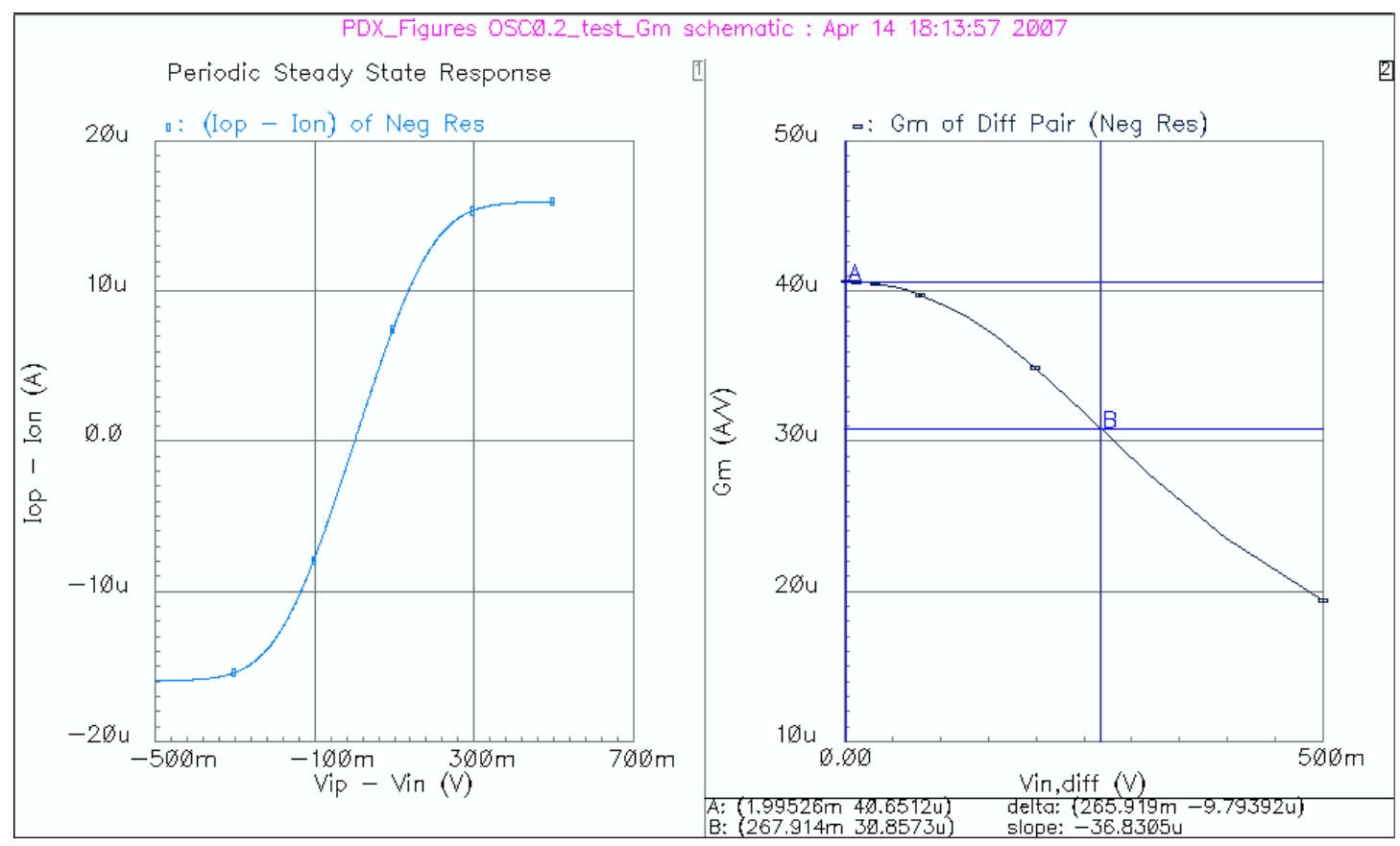

Figure 4-9 The limiting effect of the differential-pair negative resistor. (a) dc simulation results; (b) Periodical Steady State (PSS) simulation results. Markers A and B show the maximum $G_{\mathrm{M}}$ and steady-oscillator $G_{\mathrm{M}}$, respectively.

The IB terminal connects to an external biasing current $I_{\mathrm{B}}$, and generates the gate voltage for the tail-current source of the differential pair N10-N12. N10 and N12 implement a negative resistor due to their cross connection. As shown in Fig. 4-3, the differential pair doubles as a current limiter by proper transistor sizing and biasing. This is shown in Fig. 4-9. Under biasing conditions identical to its operation within the oscillator circuit, the $V-I$ transfer function of the differential pair was simulated. The de simulation shows that the output current is clipped at about $14 \mathrm{uA}$, and the $G_{\mathrm{M}}$ plot shows that as the input signal amplitude increases, the effective $G_{\mathrm{M}}$ decreases smoothly to allow for smooth oscillation amplitude settling. To make the loop gain $>1$ 
and start an oscillation, the active inductor $R_{\mathrm{p}}$ must be greater than $1 / 73 \mu \mathrm{S}=13.7 \mathrm{k} \Omega$.

By tuning $I_{\mathrm{B}}$, the oscillation amplitude can be adjusted, since $I_{\mathrm{B}}$ determines the "starving" point of $G_{\mathrm{M}}$ in the oscillation loop. However, as seen in Fig. 4-5, $I_{\mathrm{B}}$ diverts a small portion of current out of $\mathrm{N} 0$ and $\mathrm{N} 8$, and will therefore change $f_{\mathrm{R}}$ a little bit. This effect can be reduced by adjusting $I_{\mathrm{F}}$ and $I_{\mathrm{B}}$ jointly. As discussed before, if $I_{\mathrm{B}}$ is overly large, the oscillation will be voltage limited by the active inductor and the output harmonic distortion will be high.

\subsubsection{Design Considerations}

Per Eq. (4.3), it is desirable to have a higher $Q_{\mathrm{R}}$ so that the oscillator-output harmonics are low. On the other hand, a higher $Q_{\mathrm{R}}$ will increase the sensitivity to the $G_{\mathrm{M}}$ phase error. Considering both, a $Q_{\mathrm{R}} \approx 100$ is chosen for the active inductor. $Q_{\mathrm{R}}$ will be decreased by the $g_{\mathrm{ds}}$ of $\mathrm{N} 10$ and N12. If the terminal currents and voltages of the cross-connected differential pair N10 and N12 are monitored, significant phase shifts are found on their drain currents, due to the two $C_{\mathrm{gd}}$ terms. However, both can be considered as extrinsic to the two NFETs and absorbed by $C_{\mathrm{P}}$ in the active inductor (Fig. 4-2). With this treatment, when N10 and N12 are closely placed in layout, the phase shift of their intrinsic $G_{\mathrm{M}}$ is very close to $0^{\circ}$. Similarly, their $C_{\mathrm{gs}} \mathrm{S}$ and $C_{\mathrm{jd}} \mathrm{S}$ can be absorbed into $C_{\mathrm{P}}$, reducing $f_{0}$ a little bit. To keep the parasitic capacitance balanced in the differential circuit, the cross connections of N10 and N12 are made fully symmetrical in layout through the use of dummy interconnects.

To prevent the oscillator load from reducing $f_{0}$, the source followers N15 and 
N16 are placed within the oscillator layout to reduce their gate-connection lengths.

\subsubsection{Simulation Results and Analysis}

Figure 4-10 shows the transient simulation results from Cadence SpectreRFTM, for both the single-ended and differential output voltages. Figure 4-11 shows the PSS results, with $f_{0}=5.929 \mathrm{GHz}, V_{\mathrm{pp}, \text { diff }}=536.7 \mathrm{mV}$, THD $=0.6 \%(-44.4 \mathrm{~dB})$. The output current of $G_{\mathrm{M}}$, in contrast, has a THD of $6.2 \%(-24.2 \mathrm{~dB})$, showing that $G_{\mathrm{M}}$ is acting as the amplitude limiter as designed. $I_{\text {out }}$ of $G_{\mathrm{M}}$ lags the $G_{\mathrm{M}}$ input voltage $\left(V_{\mathrm{oa}}-V_{\mathrm{ob}}\right)$ by $41.7^{\circ}$, because the effect of the $C_{\mathrm{gd}} \mathrm{S}$ is included in $I_{\text {out }}$ of $G_{\mathrm{M}}$, which cannot be extracted from simulation. The output current of $G_{\mathrm{M}}$ is

$$
I_{\text {out }}=\left(g_{\mathrm{m}}-j \omega_{0} 2 C_{\mathrm{gd}}\right) V_{\text {Gate }}
$$

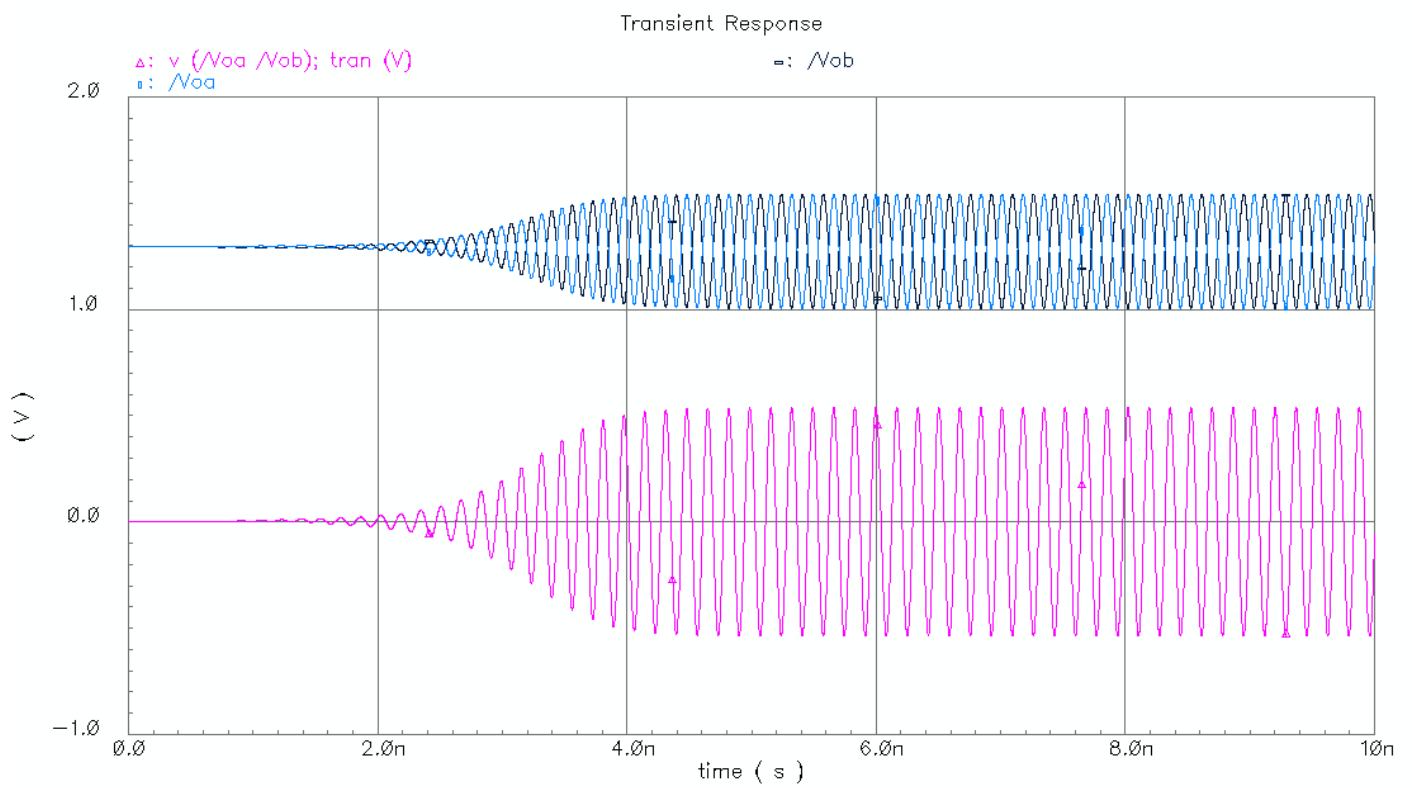

Figure 4-10 Transient simulation results of the oscillator (OSC0.2). 


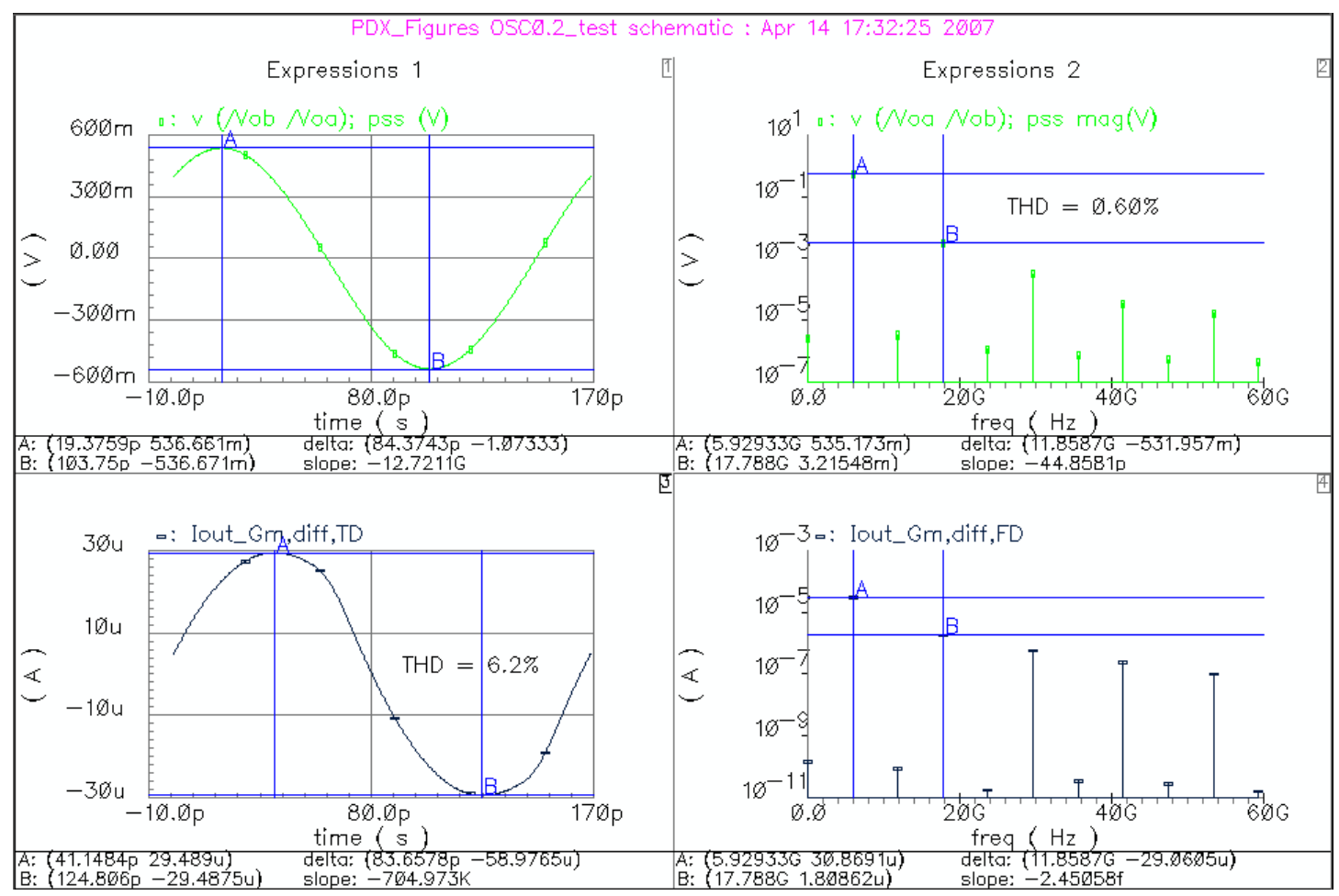

Figure 4-11 PSS simulation results of the oscillator. (1) Output voltage $V_{\mathrm{oa}}-V_{\mathrm{ob}}$ in time domain. (2) Spectrum of $V_{\mathrm{oa}}-V_{\mathrm{ob}}$. (3) Output current of differential-pair $G_{\mathrm{M}}$ in time domain. (4) Spectrum of $G_{\mathrm{M}}$ output current.

The factor of 2 arises from the fact that due to the cross connection, $V_{\text {gate }}$ and $V_{\text {drain }}$ of the $G_{\mathrm{M}}$ cell are complementary. Per the discussion in Section 4.2.2, the $C_{\mathrm{gd}} \mathrm{S}$ can be absorbed by active inductor $C_{\mathrm{p}}$ and thus the $G_{\mathrm{M}}$ phase lag is in fact much less than the apparent value $41.7^{\circ}$.

In Fig. 4-11, when dividing $I_{\mathrm{out}}=29.5 \mu \mathrm{A}$ by the $G_{\mathrm{M}}$ input voltage $V_{\mathrm{oa}}-V_{\mathrm{ob}}=$ $536.7 \mathrm{mV}$ we get the effective transconductance $G_{\mathrm{M} \text {,eff }}=54.97 \mu \mathrm{S}$, contradicting the value of $57.3 \mu \mathrm{S}$ at the signal amplitude of $236 \mathrm{mV}$, as predicted by Fig. 4-9(b). The apparent paradox is again due to the parasitic capacitances $C_{\mathrm{gd}} \mathrm{S}$ of N10 and N12, which generate an orthogonal current and inflate the observed $I_{\text {out }}$, per Eq. (4.14). Since we know that the angle of $I_{\text {out }}$ is about $41.7^{\circ}$ from simulation, we have 


$$
G_{\mathrm{M}, \mathrm{eff}}=\frac{29.5 \mu \mathrm{A} \cdot \cos 41.7^{\circ}}{536.7 \mathrm{mV}}=41 \mu \mathrm{S}
$$

very close to the Fig. 4-9(b) prediction of $40.65 \mu \mathrm{S}$.

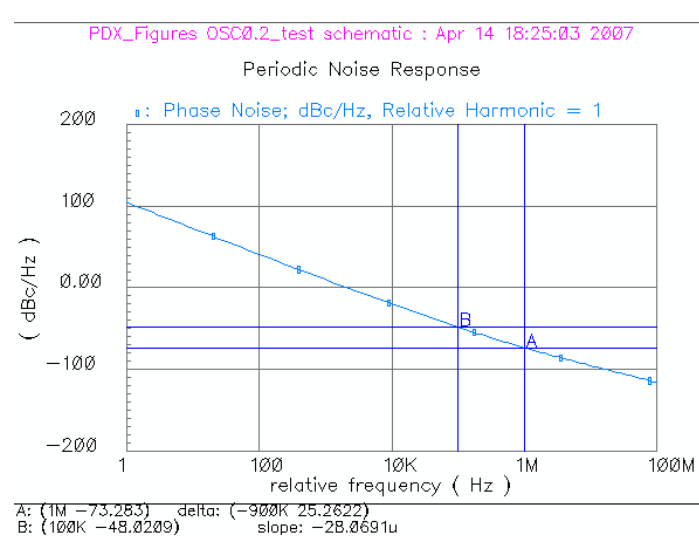

(a)

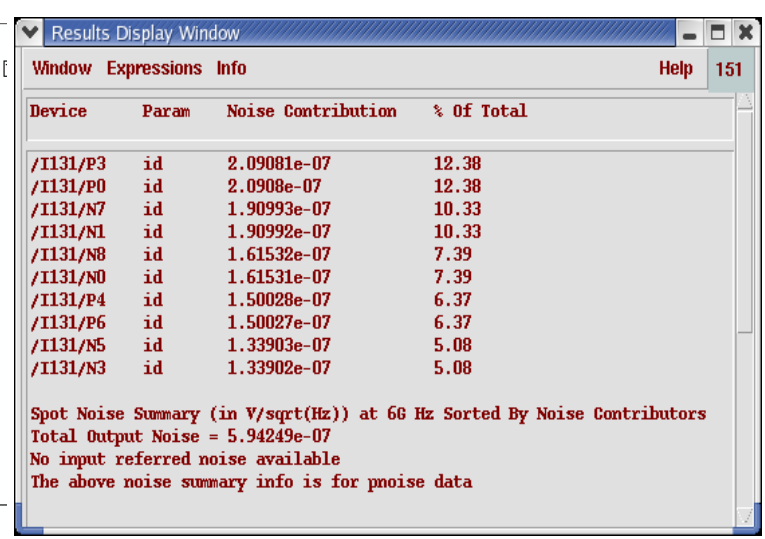

(b)

Figure 4-12 (a) Phase noise of the oscillator. (b) Noise summary.

Figure 4-12(a) shows the phase noise, which is $-73.28 \mathrm{dBc} / \mathrm{Hz}$ at $1 \mathrm{MHz}$ offset from $f_{0}$, not very good compared with passive $L-C$ oscillators, whose phase noise is around -100 to $-110 \mathrm{dBc} / \mathrm{Hz}$ at $1 \mathrm{MHz}$ offset when $f_{0}=1.6-2.4 \mathrm{GHz}$. But we will look at this issue further and see where the limitation to good phase noise comes from. Figure 4-12(b) shows the noise summary. The top ten noise contributors are all primary FETs within the active inductor, therefore, there is not much one can do to reduce the phase noise without sacrificing something else.

Table 4-1 shows the calculated versus simulation results. The small-signal parameters are extracted from the dc operating points, and are almost the same as those of the active inductor, as the dc biasing is the same. There are a few minor differences because of the addition of the negative-resistor differential pair, which modifies the circuit biasing current by a small amount. The addition of the crosscoupled differential pair does change the biasing slightly. It is found that the 
calculated $f_{0}$, oscillation amplitude $V_{\mathrm{o}, \mathrm{a}}$, and phase noise are all very close to the simulation results, confirming the validity of the analysis.

Table 4-1 Calculated versus Simulated Oscillator Parameters

\begin{tabular}{|c|c|c|c|c|c|c|c|c|}
\hline & $\begin{array}{l}\text { Para- } \\
\text { meter }\end{array}$ & Unit & Value & $\begin{array}{l}\text { Para- } \\
\text { meter }\end{array}$ & Unit & Sims & Calc'd & $\begin{array}{l}\text { Equ- } \\
\text { ation }\end{array}$ \\
\hline \multirow{9}{*}{ 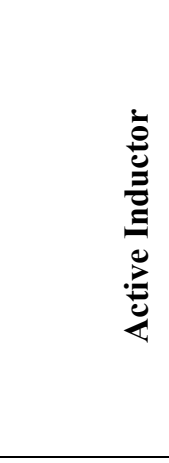 } & $g_{\mathrm{m} 1}$ & $\mathrm{mS}$ & 1.22 & GammaN & & 0.8786 & & (2.73) \\
\hline & $g_{\mathrm{m} 2}$ & $\mathrm{mS}$ & 1.09 & GammaP & & 0.9685 & & $(2.73)$ \\
\hline & $g_{\mathrm{m} 3}$ & $\mathrm{mS}$ & 1.18 & $K_{\mathrm{NF} 1}$ & & 3.6942 & & (2.69) \\
\hline & $C_{1}$ & $\mathrm{fF}$ & 19.77 & $K_{\mathrm{NF} 2}$ & & 1.8471 & & $(2.71)$ \\
\hline & $C_{2}$ & $\mathrm{fF}$ & 22.35 & & & & & \\
\hline & $C_{3}$ & $\mathrm{fF}$ & 20.30 & $I_{\mathrm{n}}$ & $\begin{array}{l}\mathrm{pA} / \\
\operatorname{sqrt}(\mathrm{Hz})\end{array}$ & 10.85 & 10.448 & $(4.9)$ \\
\hline & $g_{1}$ & $\mathrm{uS}$ & 126.16 & & & & & \\
\hline & $g_{3}$ & $\mathrm{uS}$ & 170 & $f_{0}$ & $\mathrm{GHz}$ & 5.929 & 5.930 & $(4.5)$ \\
\hline & $G$ & $\mathrm{mS}$ & 2.39 & $\omega_{0}$ & $\mathrm{Grad} / \mathrm{s}$ & & 37.26 & $(4.5)$ \\
\hline \multirow{4}{*}{ 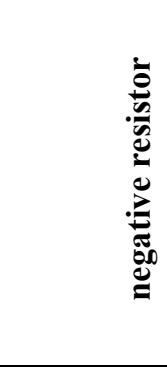 } & $\begin{array}{l}C_{1} \text { extra, } \\
\left(M_{\mathrm{i} 1}, M_{\mathrm{i} 2}\right)\end{array}$ & $\mathrm{fF}$ & 3.52 & $R_{\mathrm{p}}$ & Kohm & & 33.35 & $(4.6)$ \\
\hline & $\begin{array}{l}g_{1} \text { extra, } \\
\left(M_{\mathrm{i} 1}, M_{\mathrm{i} 2}\right)\end{array}$ & $\mathrm{uS}$ & 1.4 & $Q_{\mathrm{R}}$ & & & 28.94 & $(2.58)$ \\
\hline & $I_{\mathrm{B}}$ & $\mathrm{uA}$ & 15.8 & $V_{\mathrm{o}, \mathrm{a}}$ & $\mathrm{mV}$ & 267.6 & 263.47 & $(4.7)$ \\
\hline & $G_{\mathrm{mi}}$ & $\mathrm{uS}$ & 81.4 & $\begin{array}{l}\text { Phase } \\
\text { Noise@ } 1 \\
\text { MHz offset }\end{array}$ & $\mathrm{dBc} / \mathrm{Hz}$ & -73.28 & -74.35 & $(4.13)$ \\
\hline \multirow{2}{*}{$\begin{array}{c}\text { Adjusted } \\
\text { Values } \\
\end{array}$} & $C_{1}$ & $\mathrm{fF}$ & 23.29 & & & & & \\
\hline & $g_{1}$ & $\mathrm{uS}$ & 127.56 & & & & & \\
\hline
\end{tabular}

\subsection{Scaling of the Active-Inductor Oscillator}

The active-inductor oscillation frequency $f_{0}$ is roughly proportional to the ratio of $g_{\mathrm{m}} / C$, where $g_{\mathrm{m}}$ is the average FET transconductance, and $C$ is the total effect of $C_{\mathrm{gs}}$, $C_{\mathrm{jd}}$, interconnect, etc. If the gate-overdrive voltage $V_{\mathrm{OD}}=V_{\mathrm{GS}}-V_{\mathrm{TH}}$ is to be kept the same because $V_{\mathrm{DD}}$ is the same, we need to have $W \propto L$. Since [92]

$$
g_{\mathrm{m}}=\mu_{\mathrm{n}} C_{\mathrm{ox}} \frac{W}{L}\left(V_{\mathrm{GS}}-V_{\mathrm{TH}}\right), C_{\mathrm{gs}} \approx \frac{2}{3} W \cdot L \cdot C_{\mathrm{ox}}
$$

we have 


$$
f_{0} \propto \frac{g_{\mathrm{m}}}{C_{\mathrm{gs}}} \propto \frac{1}{L^{2}}
$$

In reality, $C_{\mathrm{gs}}$ is only about $25-30 \%$ of the total capacitance. $C_{\mathrm{jd}}$ is proportional to $W$, not to $W \cdot L \propto L^{2}$ when $V_{\mathrm{OD}}$ is fixed. For this design, all high-frequency signal interconnect widths are at the minimum required by the topology design rules (TDRs), and the dc currents they carry are all well under electron migration (EM) limits, hence within the ranges in this design, the parasitic capacitance from interconnects does not scale. The overall effect is that when $L$ increases, the $f_{0}$ reduction is less than the $1 / L^{2}$ prediction. Put this in another way, if we further reduce all gate lengths by half, $f_{0}$ will increase, but to a value less than quadruple.

To validate the $L$ scaling and provide a remedial plan in case device modeling and parasitic extractions are incorrect, the active-inductor oscillator was also designed with $L=0.4 \mu \mathrm{m}$, with the same power dissipation. The simulation results are shown in Fig. 4-13.

Another dimension of circuit scaling is power, which translates into transistor $W$. If $V_{\mathrm{DD}}$, transistor $V_{\mathrm{OD}}$ and $L$ are not changed, for the same circuit, we can double all transistor $W_{\mathrm{s}}$, make the circuit consume doubled power and occupy doubled silicon area, and reduce the noise level by $3 \mathrm{~dB}$ [1]. Alternatively, we can scale $W$ down, reducing the power dissipation and area, at the cost of a higher noise level. However, there are a few practical considerations that limit how small the oscillator can be. 


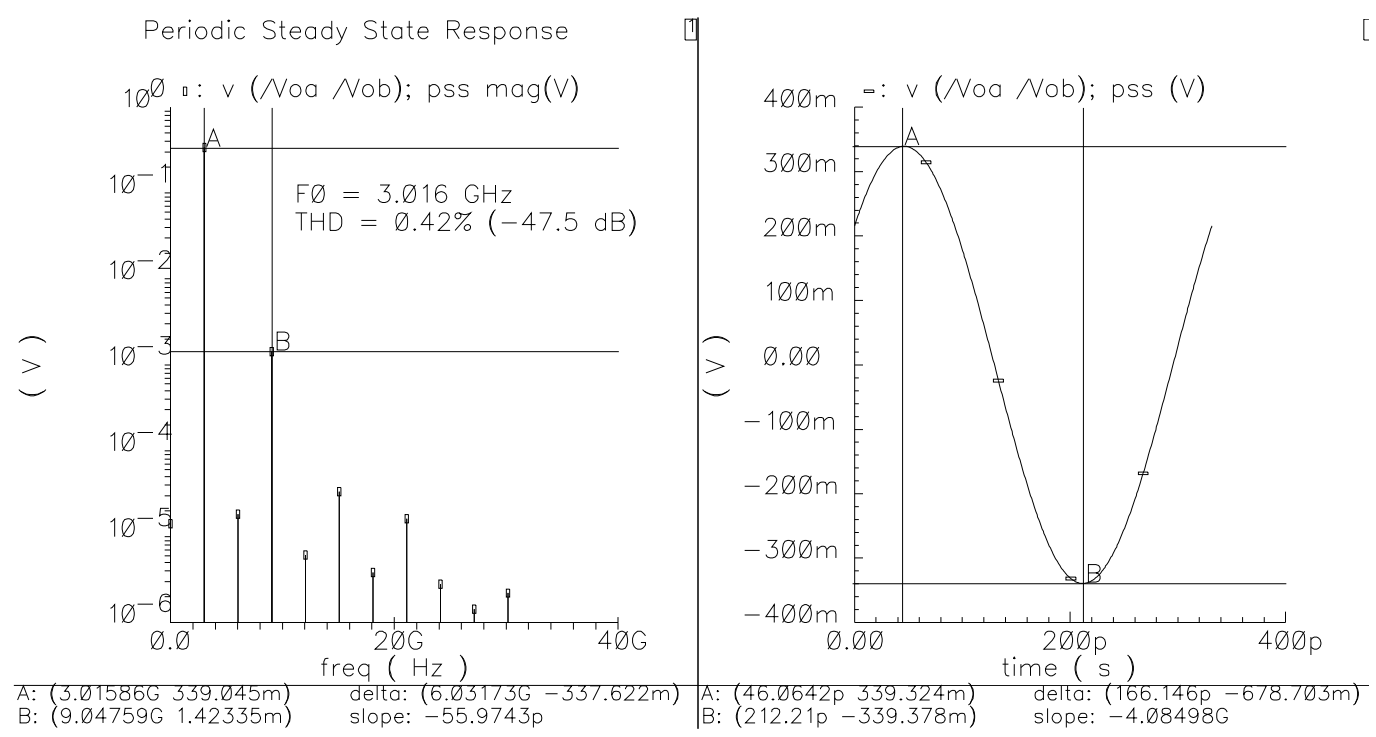

Figure 4-13 Simulation results of the $L=0.4 \mu \mathrm{m}$ oscillator (OSC0.4).

First, for each particular IC process, TDRs set a limit on the minimum circuit feature size, be it a MOSFET or connection, and consequently transistor $W$ s cannot be arbitrarily small. Second, the external loading of $50-\Omega$ from instruments does not scale and requires the same driving capability on the output buffer, thus a smaller core circuit will require a higher-performance buffer, since the driving capability of the core decreases and the input capacitance of the output buffer must reduce correspondingly. Third, the minimum-width interconnects can carry currents for MOSFETs much larger than their minimums, and the interconnect capacitance does not scale with $W$ in the operating frequency equation:

$$
f_{0} \propto \frac{g_{\mathrm{m}}}{C_{\mathrm{gs}}+C_{\mathrm{jd}}+C_{\text {interconnect }}}
$$

Thus, within a certain range $(W \approx 0.2-16 \mu \mathrm{m}$ and $L=0.2 \mu \mathrm{m}), g_{\mathrm{m}}$ scales with $C_{\mathrm{gs}}$ and $C_{\mathrm{jd}}$ linearly, with $C_{\text {interconnect }}$ being a constant, and circuits using minimum $W \mathrm{~s}$ will be penalized with a lower $f_{0}$, because $C_{\text {interconnect }}$ is a major portion in the total 
capacitance and does not change. On the other hand, if $W \mathrm{~s}$ are close to the upper limit ( $\approx 16 \mu \mathrm{m}), f_{0}$ will be higher and closer to the limit set by the active devices, but the circuit consumes more current. The OSC 0.2 example uses $W=3 \mu \mathrm{m}$ for primary NFETs, so we are closer to the low end of $f_{0}$ and did not fully explore the device potential. The reason of not using large $W \mathrm{~s}$ is that due to the high $f_{0}$, power dissipation will become excessive ( $>20 \mathrm{~mW}$ ) when $W$ s close to the high end is used.

\subsection{Conclusion}

Active inductors can be used to implement oscillators by means of a feedback scheme or negative resistor. The phase shift of the feedback scheme must be kept small, or the circuit may not oscillate at several GHz. To keep the harmonic distortion of the output voltage low, the negative resistor must be the amplitude limiter, not the active inductor. The oscillation frequency, amplitude, and phase noise of the active-inductor oscillator have been analyzed and the calculated results match the simulated results. 


\section{CHAPTER 5}

\section{IMPLEMENTATION AND LAB TESTING}

\subsection{The Need for Differential Signaling}

All filters and oscillators are designed differentially to suppress noise coupling and double the dynamic range. This is especially important at several $\mathrm{GHz}$, because the substrate and interconnects can act as a feed-forward "bridges" between filter input and output due to the parasitic capacitive coupling of the signals, as illustrated in Fig. 5-1(a). If all signals are differential and closely placed in layout, as shown in Fig. 51(b), the positive and negative signal lines will tug the local substrate in opposite directions and inject minimum capacitance-coupled currents into the substrate; thus there is very little substrate coupling. The cost is that the parasitic capacitances appearing to the differential signal nodes become larger, since they are now connected to virtual grounds instead of resistive substrate or other parts of the circuit through a metal connection.

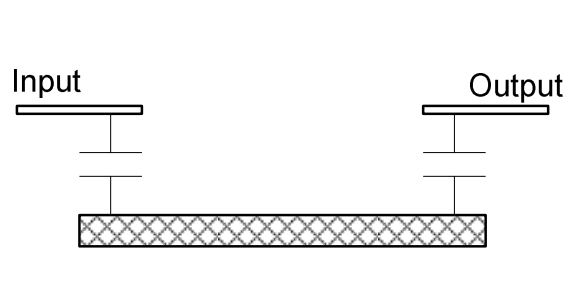

(a)

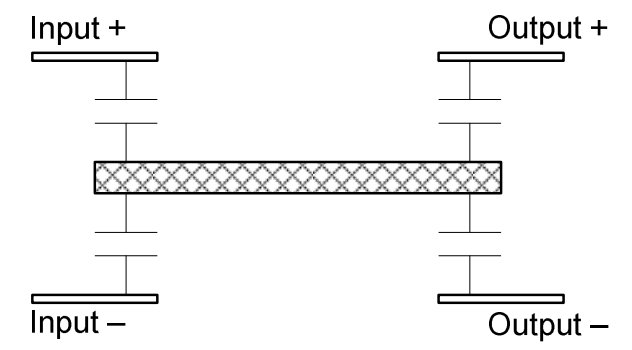

(b)

Figure 5-1 Substrate and interconnect coupling. (a) Single-ended circuit. (b) Differential circuit.

This principle in fact applies to any interconnects, such as power rails, that have to bridge two differential signals. It also applies to any signals that require isolation, not only between the inputs and outputs. The general rule is to keep differential signals 
symmetrical and reasonably close (not so close as to incur unnecessarily large parasitic capacitance); if there is any coupling to other interconnects, the coupling should be kept symmetrical, even at the cost of placing dummy metals and incurring extra capacitances.

\subsection{The Input and Output Buffers Design}

\subsubsection{The Need for Input and Output Buffers}

Since all the core circuits are designed differentially and the testing instruments are single-ended (differential Vector Network Analyzers, VNAs, are commercially available, but expensive at this time), we need to convert the input signals to the filters from single-ended to differential. This is commonly done by "baluns" at RF. Since we do not have access to differential (GSGSG, G-ground, S-signal) microprobes, to use a balun, the differential input signals will have to be connected to bondpads that will have to be accurately characterized. Further, a bondwire will have a bandpass effect due to its inductance. To mitigate the risks, we decided to use a single-ended (GSG) microprobe to inject the input signal, and design an on-chip input buffer to implement the single-ended-to-differential conversion. The input buffer must provide the proper termination resistance to the input to prevent signal reflections and the correct signal common-mode voltage to the filter-core inputs, as shown in Fig. 5-2. Ideally, the input buffer should provide a pair of differential output signals that are equal in amplitude, and out of phase by $180^{\circ}$, for all frequencies processed by the RF filter. 


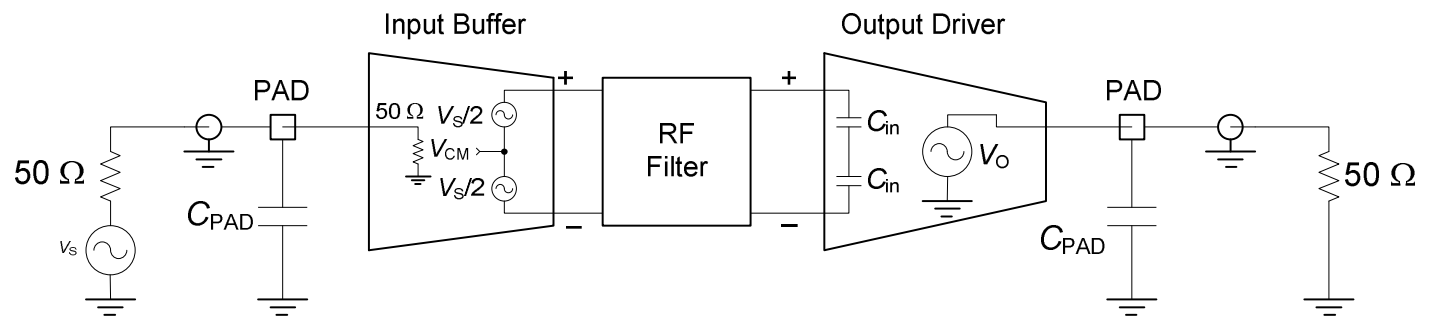

Figure 5-2 The need for input and output buffers/drivers.

An on-chip output driver is needed to convert the differential signals back into a single-ended signal, and more importantly, to drive the $50-\Omega$ load plus the large probepad parasitic capacitance $(\approx 170 \mathrm{fF})$. Its input capacitance presented to the RF filter core must be very low so as to minimize reducing $f_{0}$. Ideally, its transfer function from the inverting and noninverting inputs to the output should be equal in amplitude and phase (excluding the $180^{\circ}$ ), at all frequencies of interest to the filter.

The filters will need both the input buffer and output driver, and the oscillators will need the output driver only.

\subsubsection{The Input Buffer Design}

The most difficult part of the input-buffer design is to obtain fully symmetrical differential output signals at 1-7 GHz using only active devices, i.e., the function of a balun. Between roughly 20 and $500 \mathrm{MHz}$, transformers with double secondary winding can be used to implement baluns. For higher frequencies, microwave devices, such as the $180^{\circ}$ Hybrid [85], can be used to implement balun functions. Both can produce theoretically fully symmetrical differential outputs. However, for this design, the differential outputs cannot be fully symmetrical since only transistors can be used.

As shown in Fig. 5-3, a signal-inverting mechanism is required for the inverting 
output path. It could be part of the signal driver function or separate, but in either case, the two signal paths are not fully symmetrical, because the basic MOSFET circuitry cannot implement inverting and non-inverting functions using the same topology. The inverting output will experience more delay, and a different (usually higher) attenuation, thus for a certain sinusoidal input test signal, $V_{\mathrm{O}^{+}}$and $V_{\mathrm{O}_{-}}$will be different in amplitude and phase. The amplitude and phase differences will vary with frequency as well, as the signal-inverting block, its loading to the input and loading by the signal driver are all functions of frequency. When the differential inputs are not fully symmetrical, the filter operation is degraded due to the equivalent common-mode RF signal input. Our goal is to make the amplitude and phase differences as small as possible from 1 to $7 \mathrm{GHz}$.

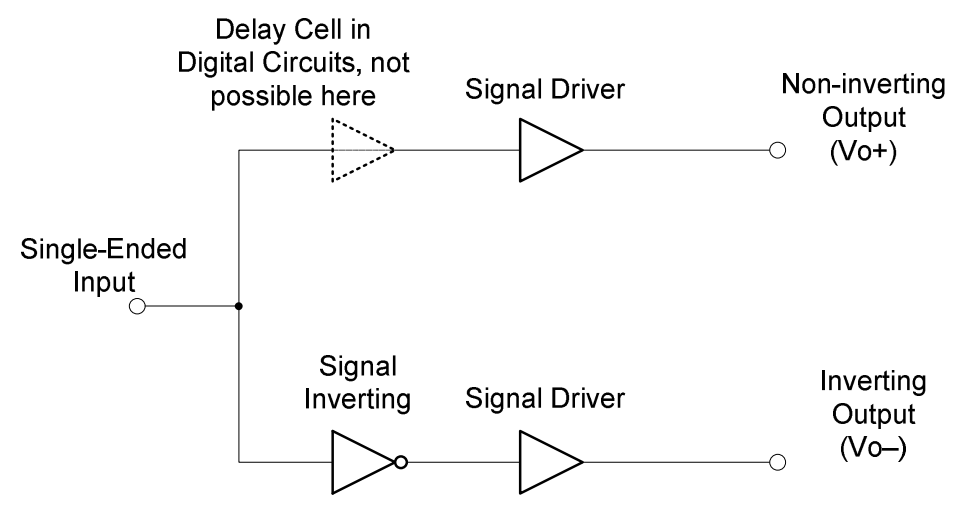

Figure 5-3 The signal path of single-ended to differential conversion.

Note that a common technique used in the digital circuitry is to insert a delay cell in the non-inverting path, as shown in Fig. 5-3, to balance the delay from the inverting block, which is a CMOS inverter. The delay cell is a CMOS pass-gate switch in the ON state, with identical MOSFETs as those of the CMOS inverter. This technique can improve the differential pulse signal matching significantly, but cannot be used here 
since the signals are sinusoidal and inverter outputs will become pulses.

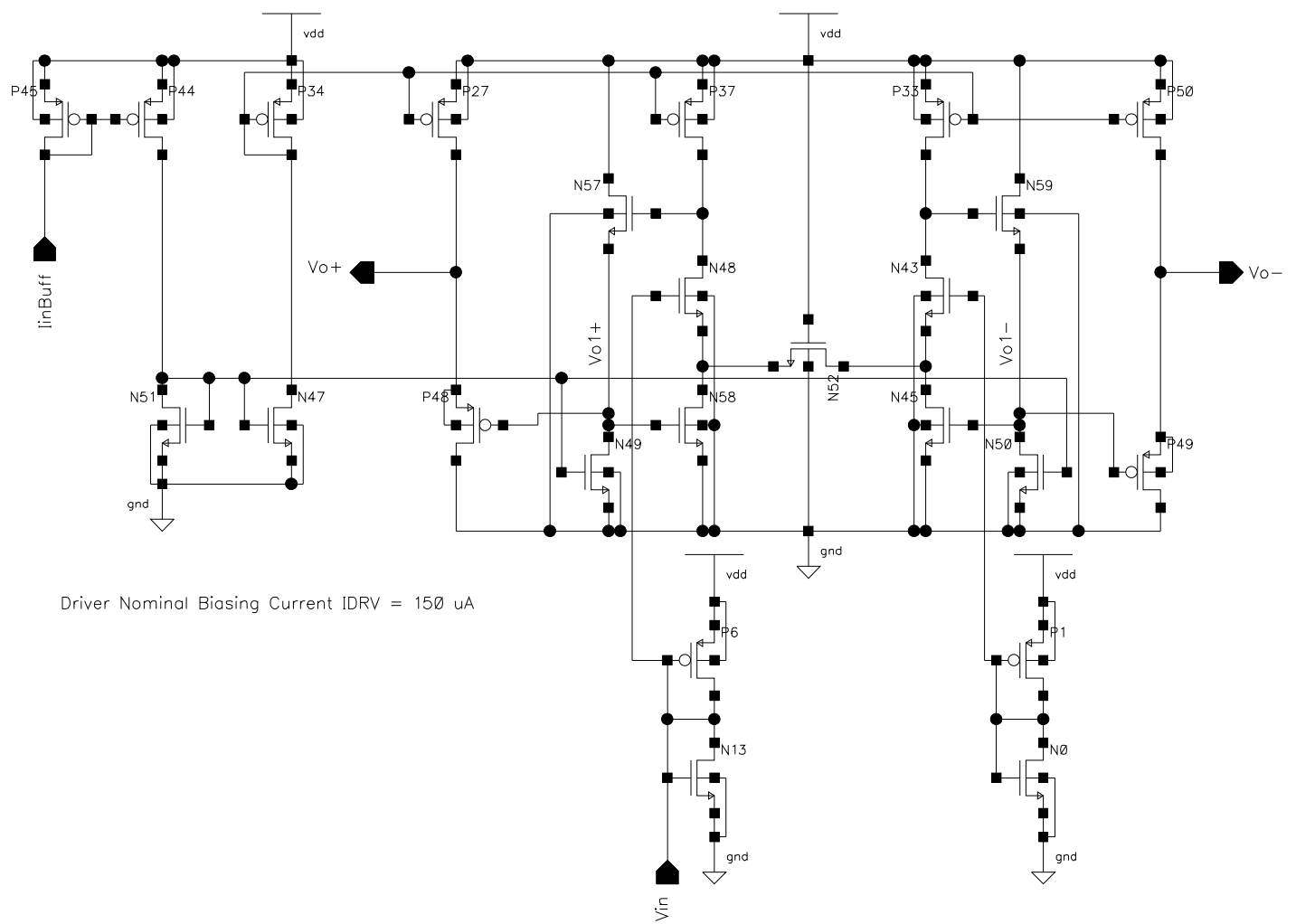

Figure 5-4 The input buffer circuit schematic.

The complete schematic of the input buffer is shown in Fig. 5-4. The input signal is connected to the drains of N13 and P6, which are both diode-connected. By proper biasing and choice of aspect ratio, N13 and P6 provide a resistance around $50 \Omega$ for input matching and a common-mode voltage of $1.1 \mathrm{~V}$ to N48 and N43. The core of the circuit is a differential transconductor [46], with one input connected to ac ground at P1 and N0. The signal-inverting function is implemented through the differential structure. N52 operates in the linear region and is a resistor. Different from the transconductor, which has NFETs mirroring the $I_{\mathrm{ds}}$ of N58 and N45, the gate voltages of N58 and N45 are buffered using source followers (P48 and P49) as output voltages. P48 and P49 also provide the desired common-mode voltage of $1.35 \mathrm{~V}$ to the filter. 


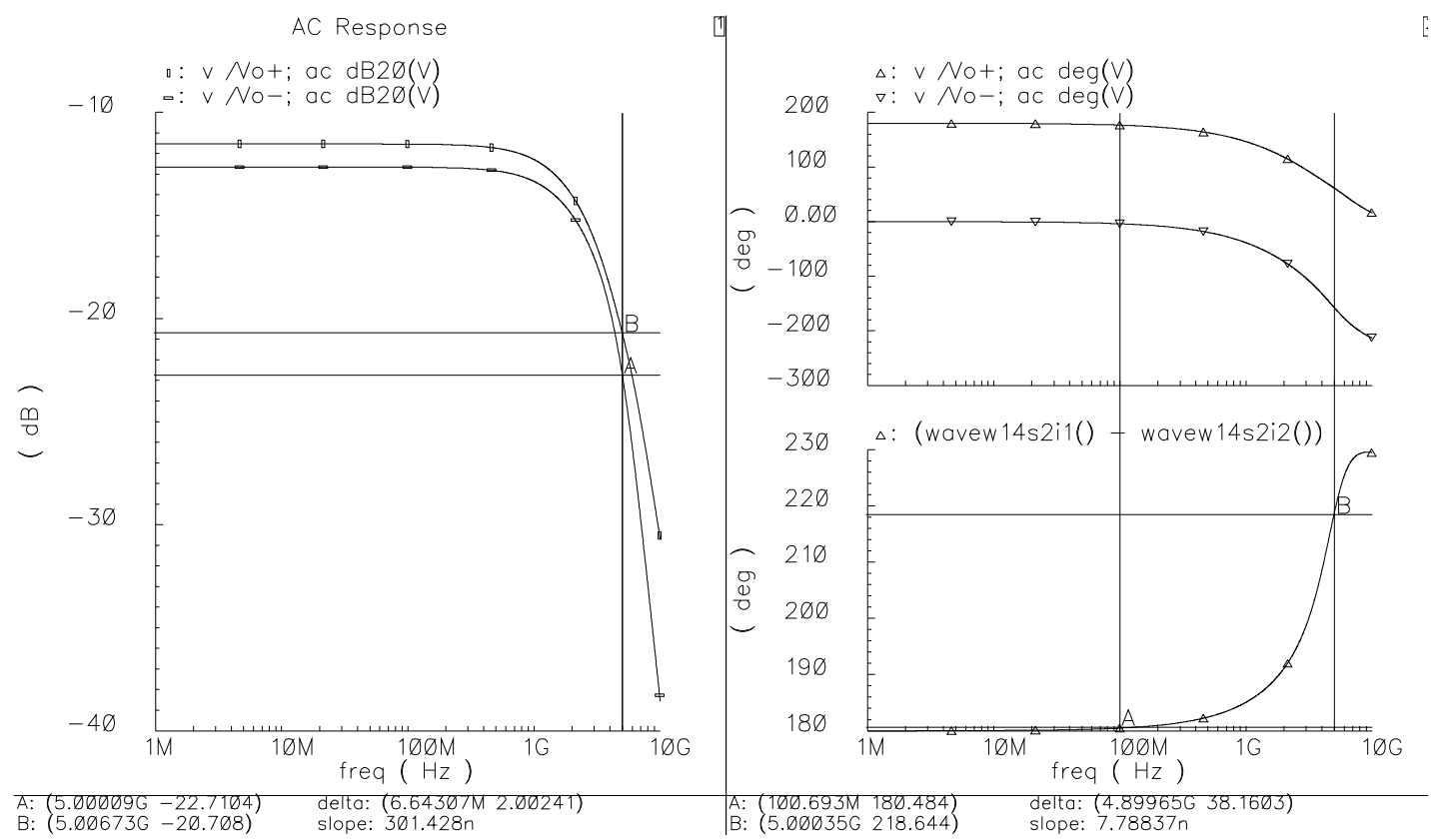

Figure 5-5 ac simulation results of the input buffer: magnitude (left) and phase (right).

This circuit is chosen mainly for its symmetry of the differential outputs. The simulation results are shown in Fig. 5-5. The two output magnitude and phase differences at $5 \mathrm{GHz}$ are $2 \mathrm{~dB}$ and $38^{\circ}$, respectively, not ideal, but close enough and are the best among the possible choices. The balun function design is non-trivial due to the high operating frequency.

\subsubsection{The Output Buffer Design}

The fundamental issue in designing the output buffer is to obtain some voltage gain, or rather, an acceptable level of attenuation, at 1-7 GHz, when driving a load of $50 \Omega$ plus a capacitance of a few hundred $\mathrm{fF}$ with a low $C_{\text {in }}$ of less than $10 \mathrm{fF}$. To a lesser extent, the individual transfer functions from the two differential inputs to the single output should have equal amplitude and be $180^{\circ}$ out of phase, and the gain profile should be as flat as possible from 1 to $7 \mathrm{GHz}$. MOSFETs need gate voltage $\left(V_{\mathrm{gs}}\right)$ to 
generate output current, and in turn, gain. However, at these frequencies, the load, $\left|1 /\left(j \omega C_{\mathrm{gs}}\right)\right|$ becomes very low, and hardly any voltage can be generated; hence it is difficult to obtain gain from cascade stages.

A $10 \mathrm{M}-18 \mathrm{GHz}$ dc blocker will be inserted in the output path, so the $50-\Omega$ load will not draw de currents due to a non-zero signal common-mode voltage. Passive inductors cannot be used to neutralize the load capacitance and expand the bandwidth through "shunt peaking" $[16,83]$ because the resulting buffer will be narrow-band and inductors are not available in the TSMC LOG018 process. A wideband buffer is required since a large simulation-silicon discrepancy is expected and we do not know beforehand what $f_{0}$ shift to expect.

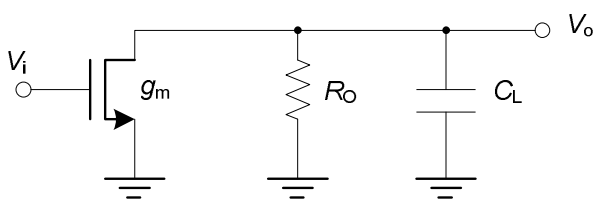

(a)

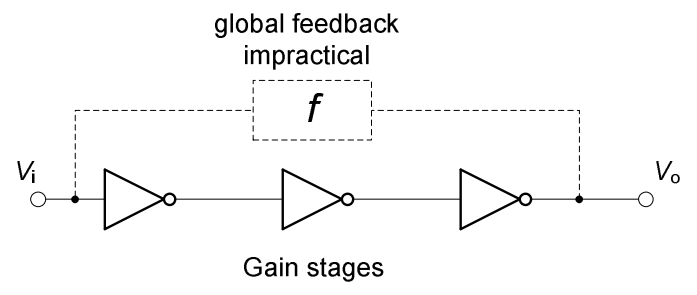

(b)

Figure 5-6 (a) A single gain stage. (b) Cascaded gain stages.

Figure 5-6(a) shows the simplified equivalent circuit of a single-amplifier stage. The FET has to be in CS configuration, since CG or CD configurations have less gain. The output is loaded with $R_{\mathrm{O}}$, which includes $g_{\mathrm{ds}}$ terms, but more likely, also an intentional small resistance from other transistors. The transfer function is

$$
T(s)=\frac{g_{\mathrm{m}} / C_{\mathrm{L}}}{s+\frac{1}{R_{\mathrm{o}} C_{\mathrm{L}}}}
$$

and two examples are shown in Fig. 5-7. A lower $R_{\mathrm{O}} \approx 1 / g_{\mathrm{m}}$ will yield a wider bandwidth $\left(f_{-3 \mathrm{~dB}}\right)$ but also incur some extra parasitic capacitance because the $C_{\mathrm{jd}}$ from 
a current-source load will become $C_{\mathrm{gs}}$ or some other larger capacitance, but it will never increase the absolute gain at frequencies from 1-7 GHz. However, relatively low $R_{\mathrm{O}}$ has to be chosen because otherwise the de gain will be too high, and possibly saturate the dc operating point of the driver when multiple stages are cascaded, as illustrated in Fig. 5-6(b).

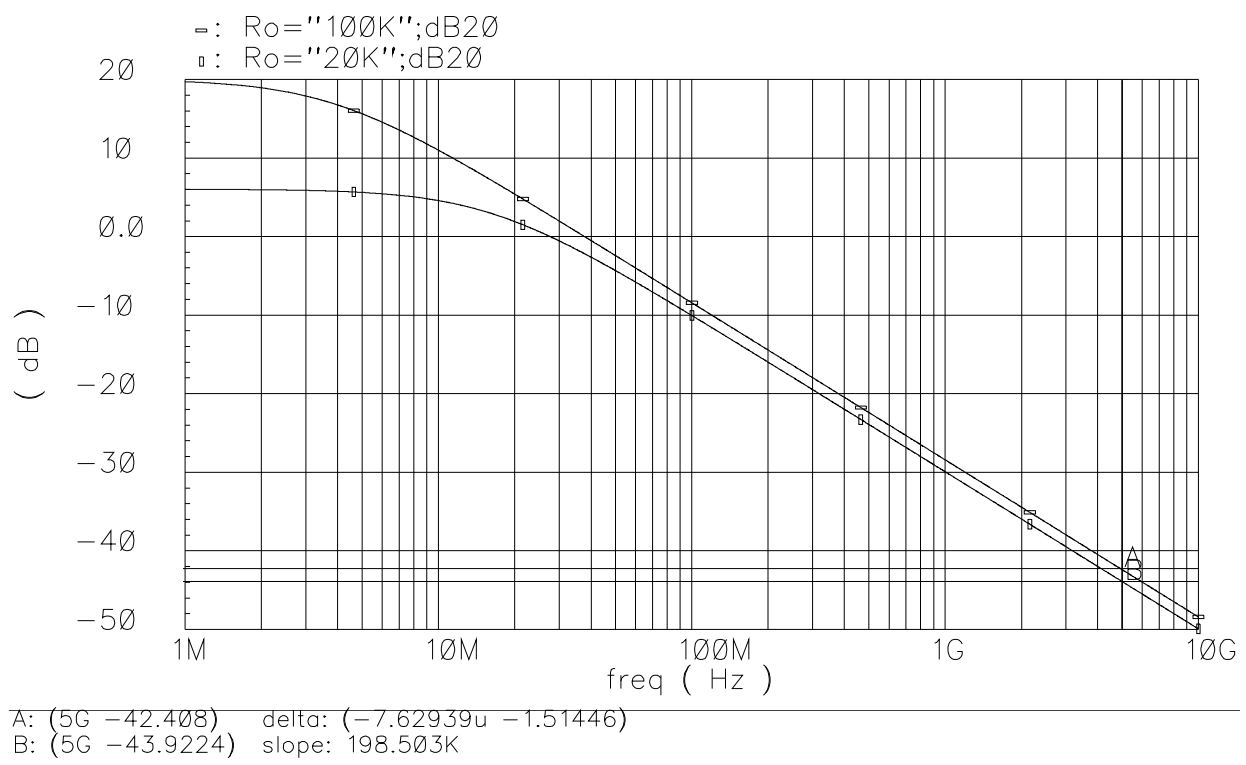

Figure 5-7 The frequency response of the circuit in Fig. 5-6(a). $C_{\mathrm{L}}=500 \mathrm{fF}$ and $R_{\mathrm{O}}=20 \mathrm{k} \Omega ; C_{\mathrm{L}}=420$ $\mathrm{fF}$ and $R_{\mathrm{O}}=100 \mathrm{k} \Omega$.

To increase the absolute gain at $5 \mathrm{GHz}$, we can increase $g_{\mathrm{m}}$, but that means a larger transistor has to be used, and presenting a larger load to the filter core. Or we can cascade a few stages, to boost the driving capability, but the attenuation at $5 \mathrm{GHz}$ will increase, because the voltage gain of each stage at $5 \mathrm{GHz}$ is less than 1 . Therefore, a delicate balance between keeping $C_{\text {in }}$ low and using more stages has to be stricken.

One may suggest that high-dc-gain stages be used, and then using feedback to make the gain profile flat and expand the $f_{-3 \mathrm{~dB}}$, as shown in Fig. 5-6(b). This proves to be impossible to implement at $1-7 \mathrm{GHz}$, as stability cannot be maintained due to the 
phase shift of the circuits at these frequencies. Local feedback was judiciously used in the final circuit.

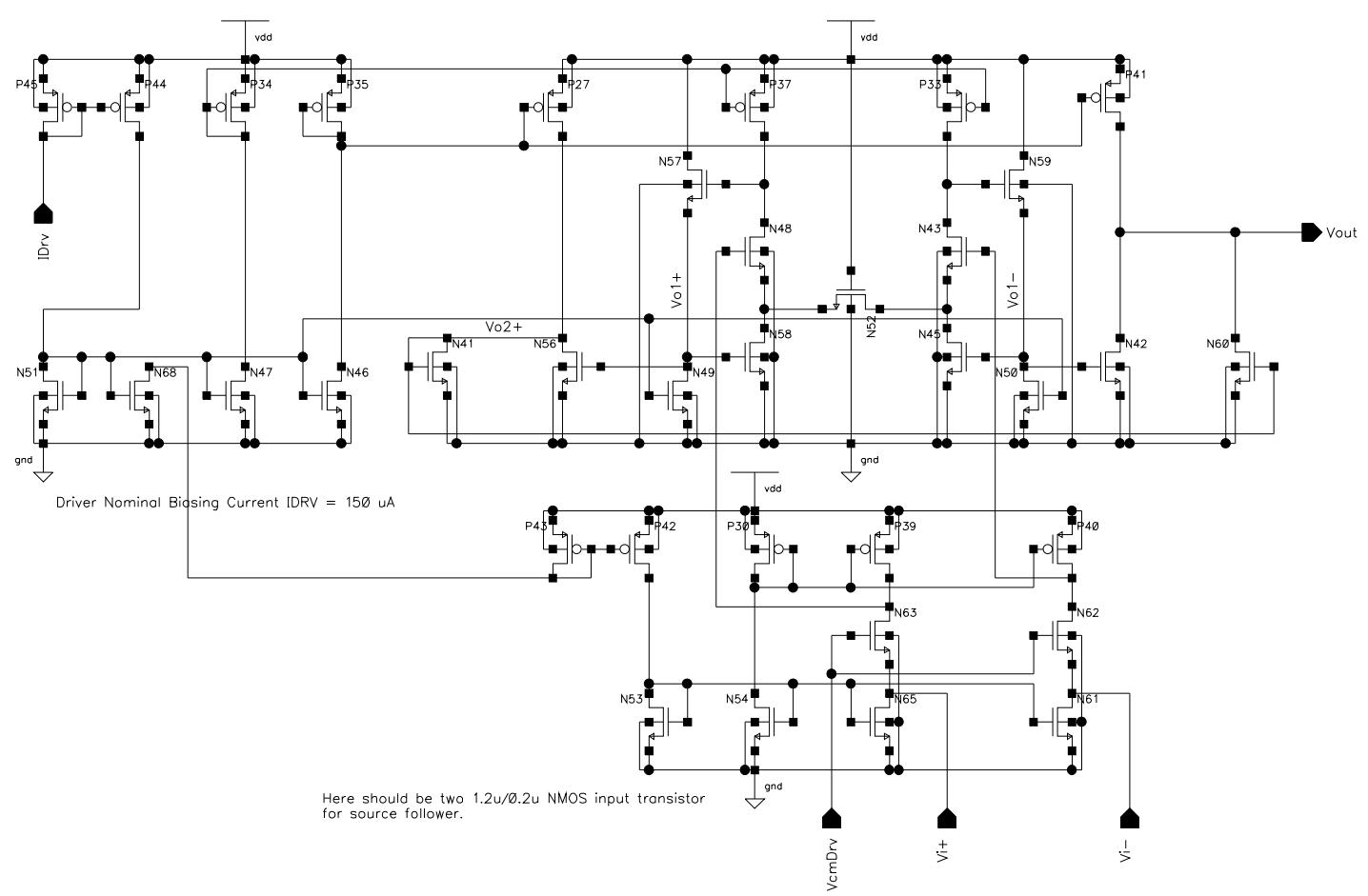

Figure 5-8 The output-driver circuit schematic.

The output-driver circuit is shown in Fig. 5-8. Current sources N65 and N61 are the load of the source followers, and cascode devices N63 and N62 convert the source follower voltages into currents into the $C_{\text {in }}$ of the driver core. The driver core is again a transconductor [46], with one of the outputs mirrored back to the same node as the other output via N41 and N60. The only internal node of the signal-inverting circuit, $V_{\mathrm{o} 2+}$, is low-impedance, to ensure that its pole frequency is far higher than the dominant pole at $V_{\text {out }}$. 


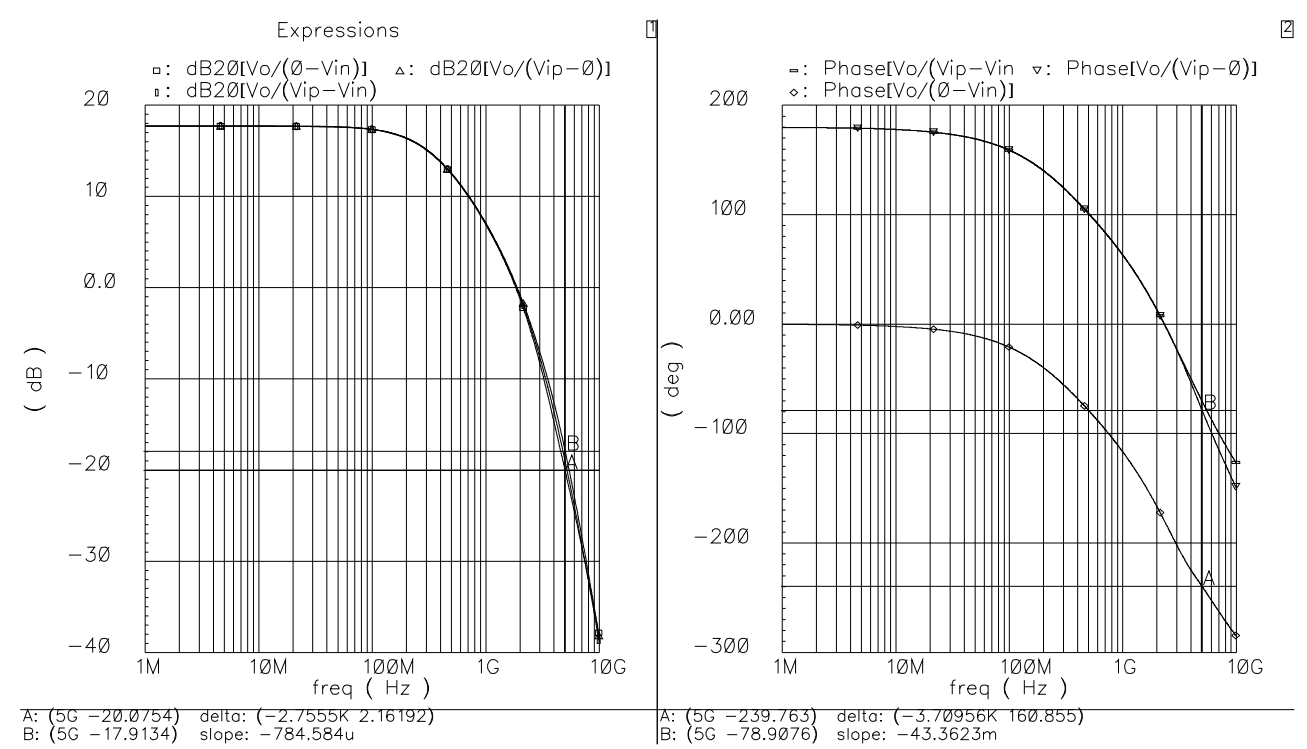

Figure 5-9 The simulation results of the output driver. Panel 1: cursors A and B are gains from singleended input on the $V_{\text {ip }}$ and $V_{\text {in }}$ terminals, respectively. The differential-input gain, $V_{\mathrm{o}} /\left(V_{\text {ip }}-V_{\text {in }}\right)$, is $-19.05 \mathrm{~dB}$.

The ac simulation results of the output driver are shown in Fig. 5-9. It is seen that the gain difference and phase errors at $5 \mathrm{GHz}$ for the two differential input terminals are $2 \mathrm{~dB}$ and $20^{\circ}$, respectively, and the gain at $5 \mathrm{GHz}$ is about $-19 \mathrm{~dB}$.

Similar to the input buffer, the output driver is not ideal. It was selected based on the stringent requirements and various tradeoffs.

\subsection{Circuit Modules Design}

Due to the lack of confidence in the available device models and parasitics-extraction data, the active-inductor filter and oscillator are both designed in several different versions: gate length $L=0.2 \mu \mathrm{m}$ and $0.4 \mu \mathrm{m}$, regular $Q$ and higher $Q$. As an example, Fig. 5-10 shows the regular- $Q 0.2-\mu \mathrm{m}$ bandpass filter module (BP0.2), which consists of three cascade blocks: the input buffer, the filter, and the output driver. 


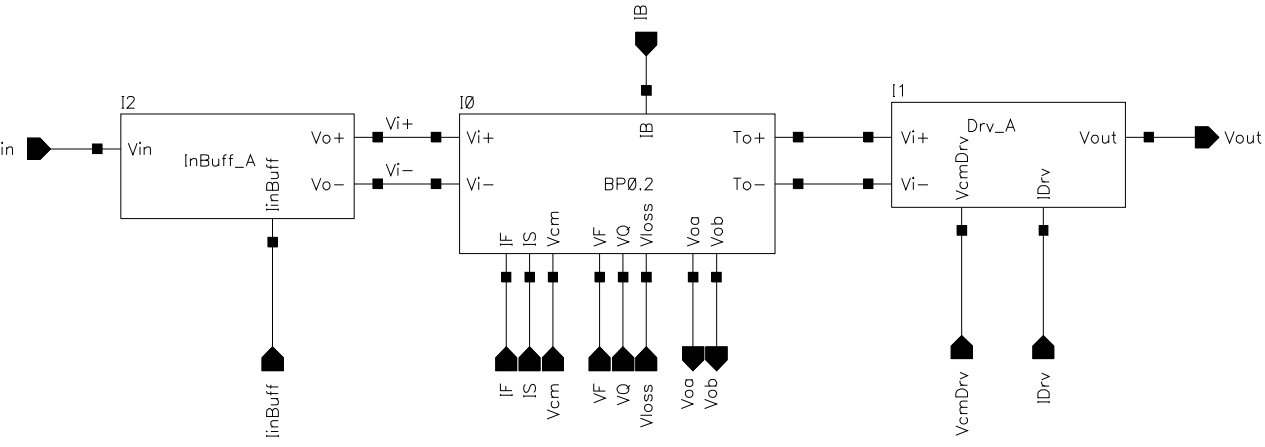

Figure 5-10 Regular- $Q$ 0.2- $\mu \mathrm{m}$-gate-length bandpass-filter module (BP0.2).

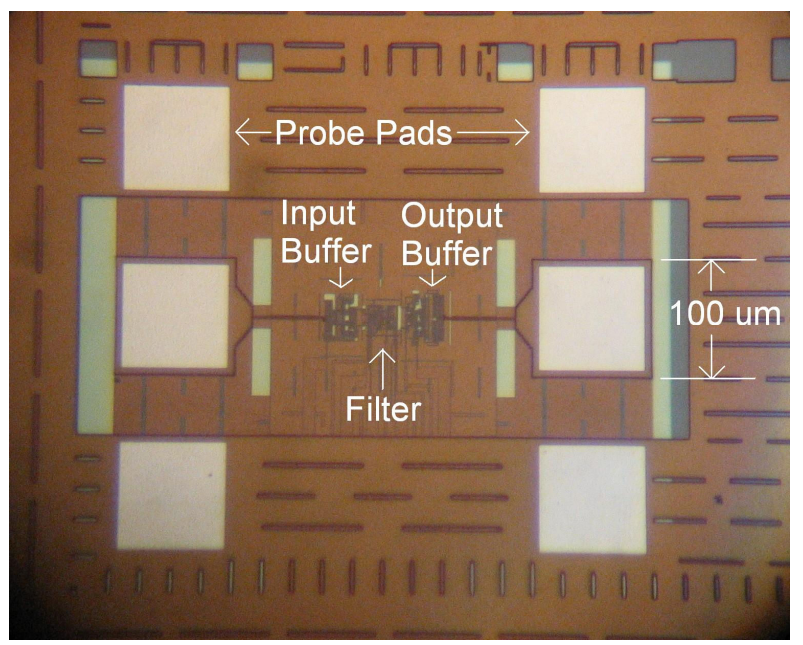

(a)

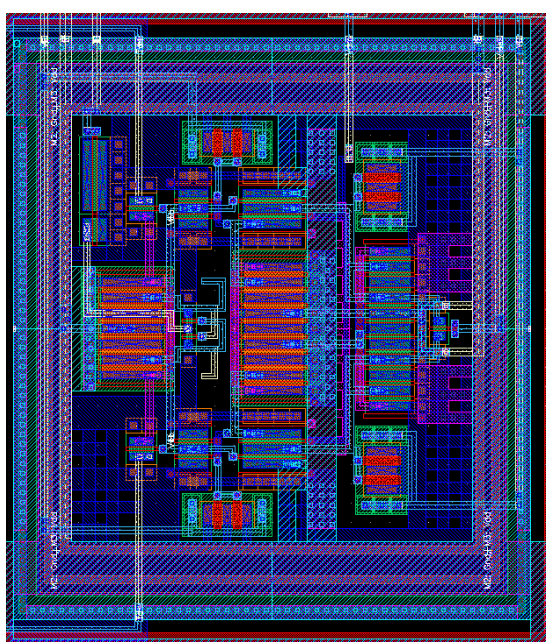

(b)

Figure 5-11 (a) The 0.2- $\mu$ m-bandpass-filter (BP0.2) module layout (microphotograph). (b) The BP0.2 filter-core layout (screen capture).

The module's layout is shown in Fig. 5-11. The active-inductor filter core occupies $26.6 \mu \mathrm{m} \times 30 \mu \mathrm{m}$, including biasing and tuning transistors. In contrast, a spiral inductor alone typically occupies $300 \mu \mathrm{m} \times 300 \mu \mathrm{m}$. In doing the circuit layout, the following considerations should be attended to simultaneously:

1. Parasitic capacitance should be minimized to maximize the operating frequency.

The layout should be generally compact; however, there is a tradeoff between reducing length of interconnects and reducing the lateral capacitance between metal traces. Since the capacitance is inverse proportional to the spacing, high- 
frequency trace spacing is maintained at twice or more the TDR minimum when possible.

2. To reduce drain-diffusion capacitance, MOSFET drains should be shared whenever possible.

3. Device and interconnect layouts are symmetrical for the differential circuit. Due to the low device count, generally a common-centroid method cannot be used, but symmetry should be maintained. Dummy poly strips should guard peripheral transistors to ensure that their gates are similar to those of the internal ones [93].

4. Metal widths should be carefully evaluated for each connection: the width should be low to reduce parasitic capacitance, but should be wide enough to comply with electron migration rules, and more frequently, not to introduce any excess dc voltage drop.

5. Long signal wires should be routed using top metal layers to reduce their capacitance to the substrate. Metal isolations may be used to decouple signals.

It is noted that all of the above considerations are tradeoffs and should be evaluated on a case-by-case basis. There are no fixed rules as to what is best.

\subsection{Chip Level Design}

Since each active-inductor oscillator or filter module has about 9 biasing terminals, 10 modules would need a 90-pin package. PGA (pin grid array) packaging can provide more than 90 pins, but it requires expensive mounting and PCB (printed circuit board) technologies. To keep the logistics work simple, a 40-pin dual-in-line package (DIP40) is used, and the biasing pins are multiplexed among the modules, as shown by 
the chip-level schematic in Fig. 5-12. The chip-level schematic is also important for layout verification. As shown in Fig. 5-13 (a), to satisfy the layer-density requirement by the foundry and create decoupling capacitance, the power and biasing nets need to be expanded and blanket the entire chip, for poly and all 6 metal layers. Due to the vast variation in layout scale between filter/oscillators cores and the entire chip, shortor open-circuit mistakes often happen, and LVS (layout versus schematic) checker will report these as discrepancies, even though locating a short-circuit place is still not easy and requires experience.

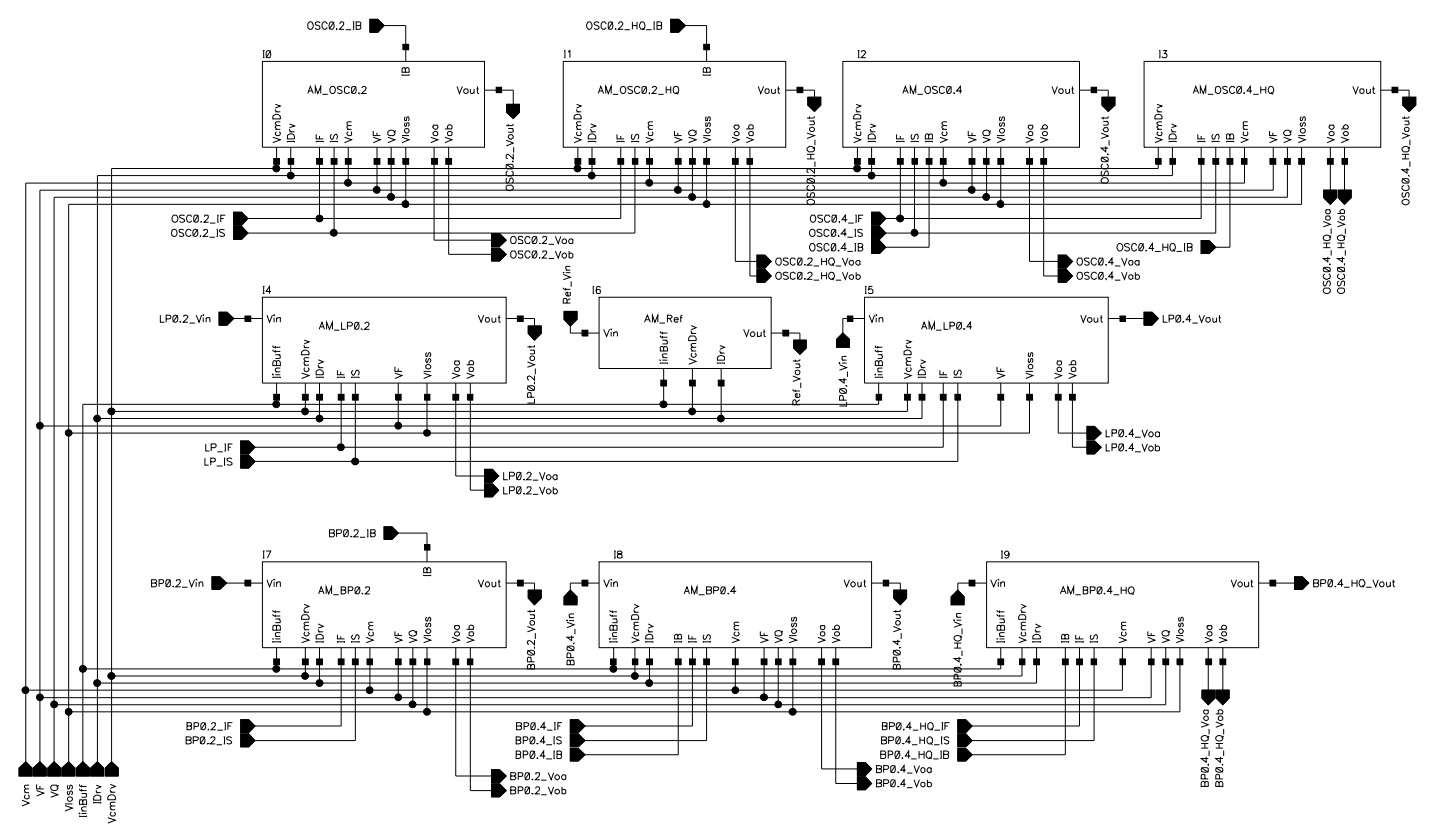

Figure 5-12 The chip-level schematic. 


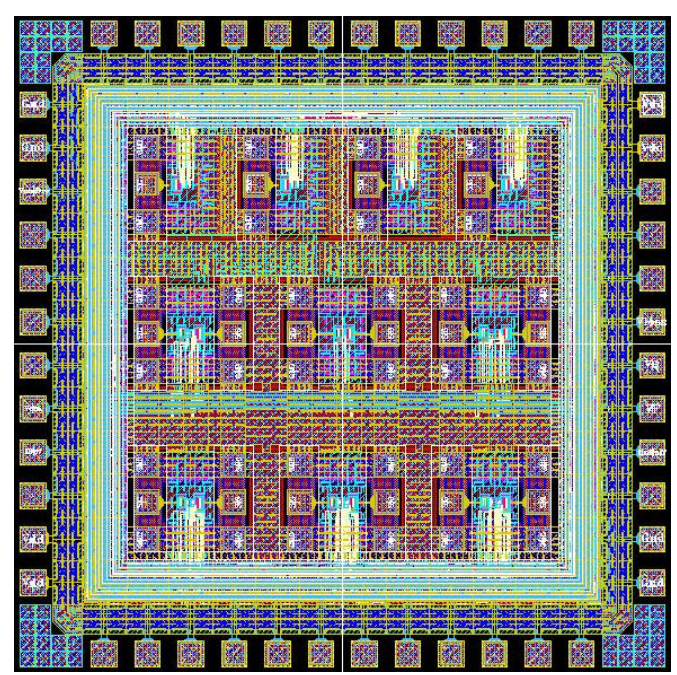

(a)

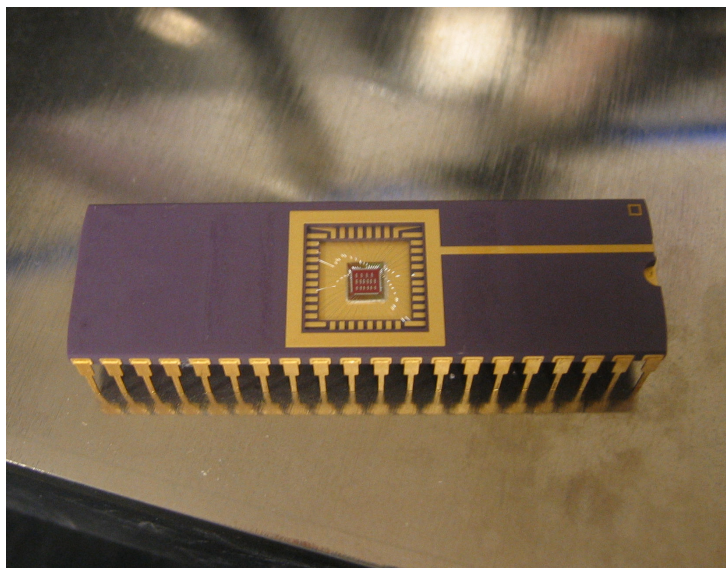

(b)

Figure 5-13 (a) The test-chip layout including probe pads and bond pads. (b) The packaged test chip (1 out of a lot of 40).

The chip-level design involves the following aspects:

1. Floorplanning: the test chip area is $7 \mathrm{~mm}^{2}$ and needs to be shared among bond pads, power rails, test modules including their probe pads, and separation between the test modules. These layout elements need to be properly organized to allow for an orderly implementation of various requirements.

2. Bondpads and probe pads: there were no library pads by the time of tapeout, so all pads are created manually. The pads are stacks of every available metal layers stitched together by vias at a density and pattern dictated by the foundry. The signal pads of RF microprobe pads contain only the top-layer metal (metal6) to reduce their parasitic capacitance to the substrate, but need to have a metal1 ground shield to decouple them from the substrate. All bond pads have ESD (electro-static discharge) protection diodes which are located beneath the power rails. 
3. Power and signal busses: they need to be sufficiently wide to reduce the dc voltage drop. The busses are arranged in concentric rings to even the voltage drop and reduce connection resistance.

4. Layer density compliance: all physical layers (poly, metals 1-6) need to occupy a pre-defined percentage of the overall reticle as required by the chemical planarization step during fabrication. This is done by expanding the power and ground nets, with an added benefit of noise filtering.

5. Wide metal stress and separation rules: to prevent mechanical stress and damages during thermal cycles, metals spanning more than $25 \mu \mathrm{m}$ in either the $\mathrm{x}$ or the y direction need to have slots in between. The minimum spacing is doubled when either adjacent metal strip is wider than twice the minimum, to reduce the chances of short circuits. These two design rules are not implemented in the tool package and have to be manually added to the rule files.

\subsection{Biasing and Lab Testing}

As shown in Fig. 5-14, a small daughter-PCB board is designed to hold the test chip under the microscope. A 3-foot-long 40-wire-wide flat cable is used to connect the daughter board to a mother board, which contains a number of switches and potentiometers to allow quick permutations of the test setup for the different modules. 25 -turn precision potentiometers are used for generating biasing voltages and currents, which are monitored by a multimeter, so that currents and voltages can be adjusted with resolutions up to $0.1 \mu \mathrm{A}$ and $0.1 \mathrm{mV}$, respectively. 


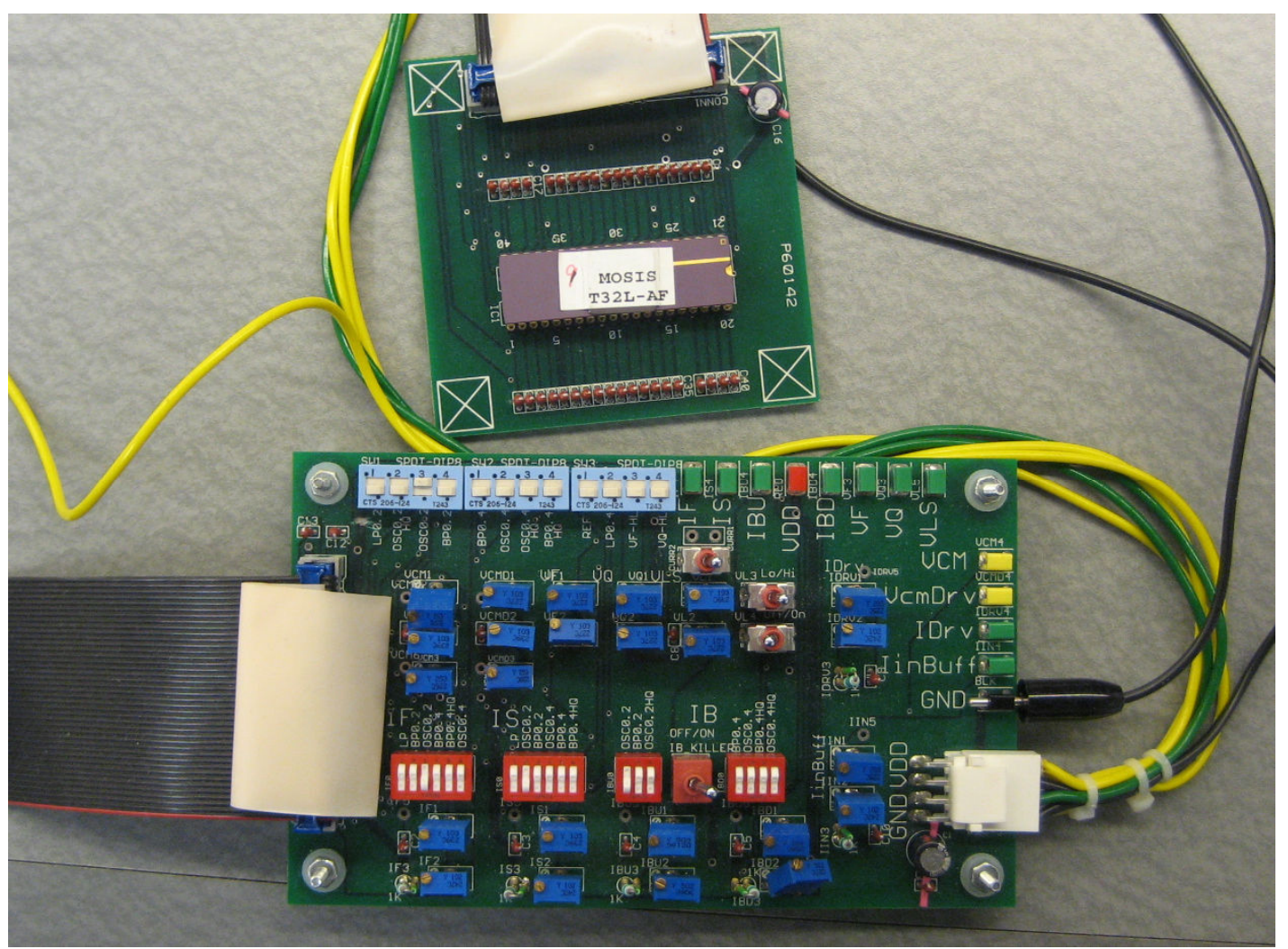

Figure 5-14 Biasing circuit for the test chip. The small circuit board carries the test chip and goes under the microscope. The larger circuit board generates tunable biasing voltage and current.

Microprobes are used to gain access to the test circuit modules for RF signals and connect them to the appropriate instruments, such as a VNA (vector network analyzer) and spectrum analyzers.

\subsection{Test Results}

\subsubsection{5-GHz Active Inductor Oscillator (OSC0.2, $L=0.2 \mu \mathrm{m})$}

Figure 5-15 shows the output spectrum of a 5-GHz oscillator, at the same nominal biasing as the active inductor in Fig. 2-29, with $I_{\mathrm{B}}=80 \mu \mathrm{A}$. The oscillation frequency of $5.645 \mathrm{GHz}$ is about $91 \%$ of the simulation results as we shall see below. The phase noise is $-66.6 \mathrm{dBc} / \mathrm{Hz}$ at $1-\mathrm{MHz}$ offset, measured with an Agilent ${ }^{\mathrm{TM}}$ 8562EC 
Spectrum Analyzer. The oscillator module is simulated at the same biasing conditions with output buffer and load, as shown in Fig. 5-17. The simulation results are shown in Fig. 5-18 for comparison.

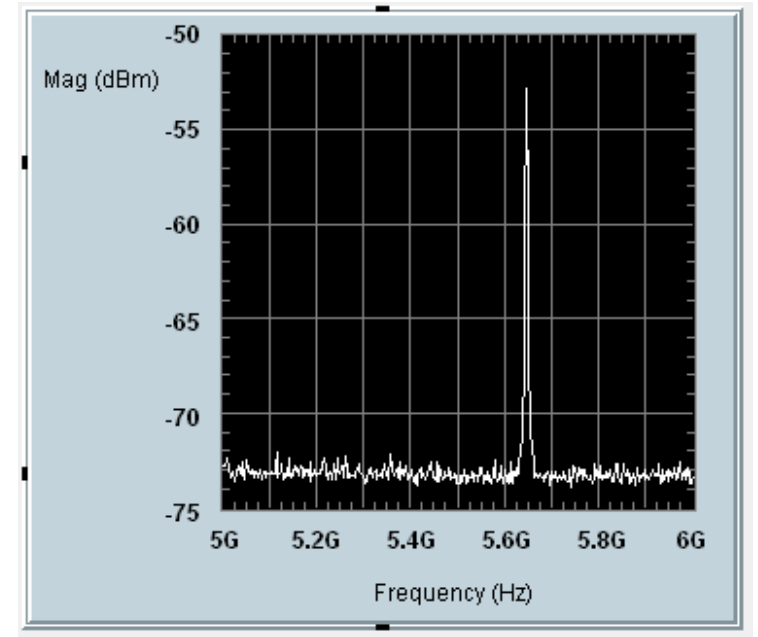

Figure 5-15 Output spectrum of a 5.6-GHz oscillator (including a 50- $\Omega$ output driver). The center frequency is $f_{0}=5.645 \mathrm{GHz}$, the magnitude is $-52.78 \mathrm{dBm}$, corresponding to a peak voltage of $134 \mathrm{mV}$ at the circuit core. The attenuation of the output buffer at $5.645 \mathrm{GHz}$ is $39.3 \mathrm{~dB}$. The 2nd and higher harmonics are below the noise floor $(\approx-73 \mathrm{dBm})$. (Data Ref\# OSC0.2-C22-SPA00040)

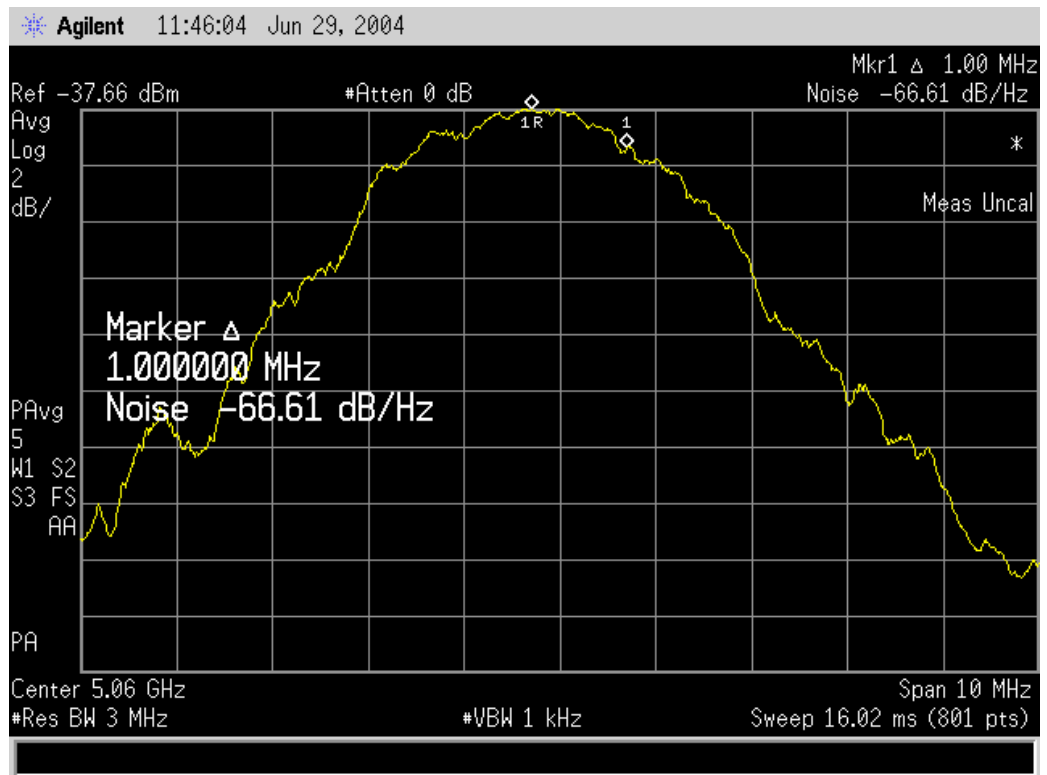

Figure 5-16 Measured phase noise of the 5-GHz oscillator, including the output buffer. 


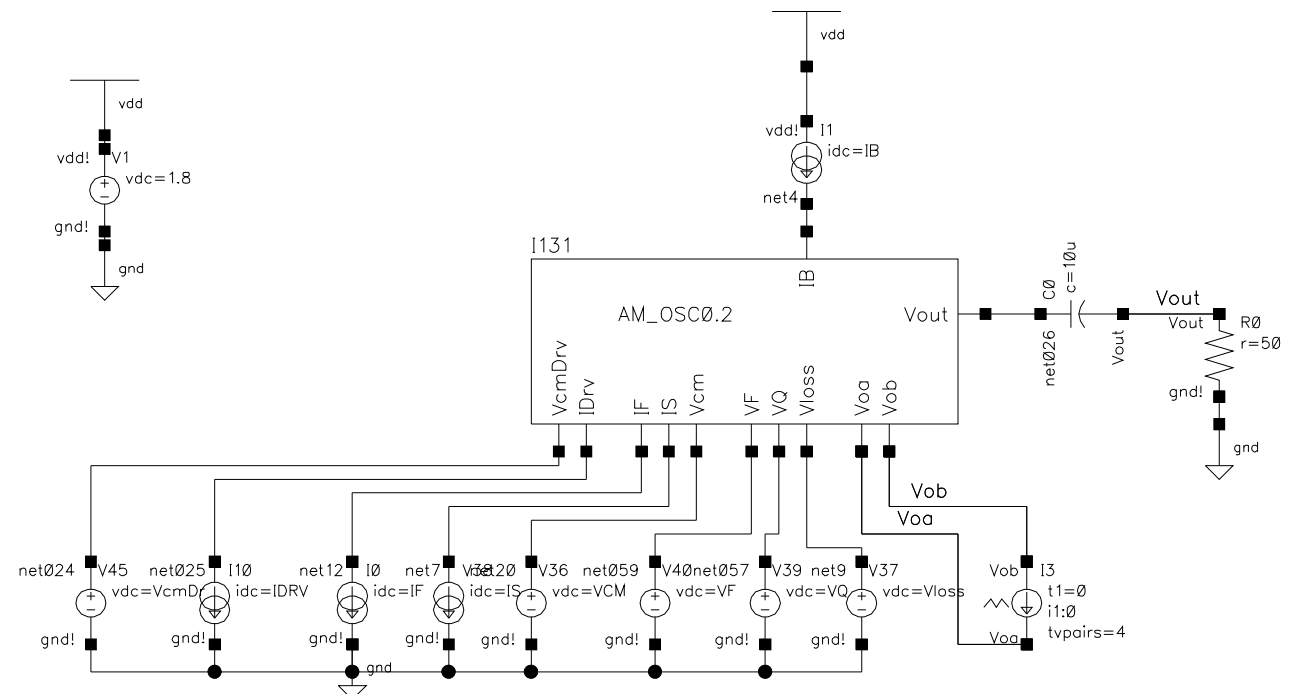

Figure 5-17 Test-bench configuration for the 5-GHz oscillator $(L=0.2-\mu \mathrm{m})$.

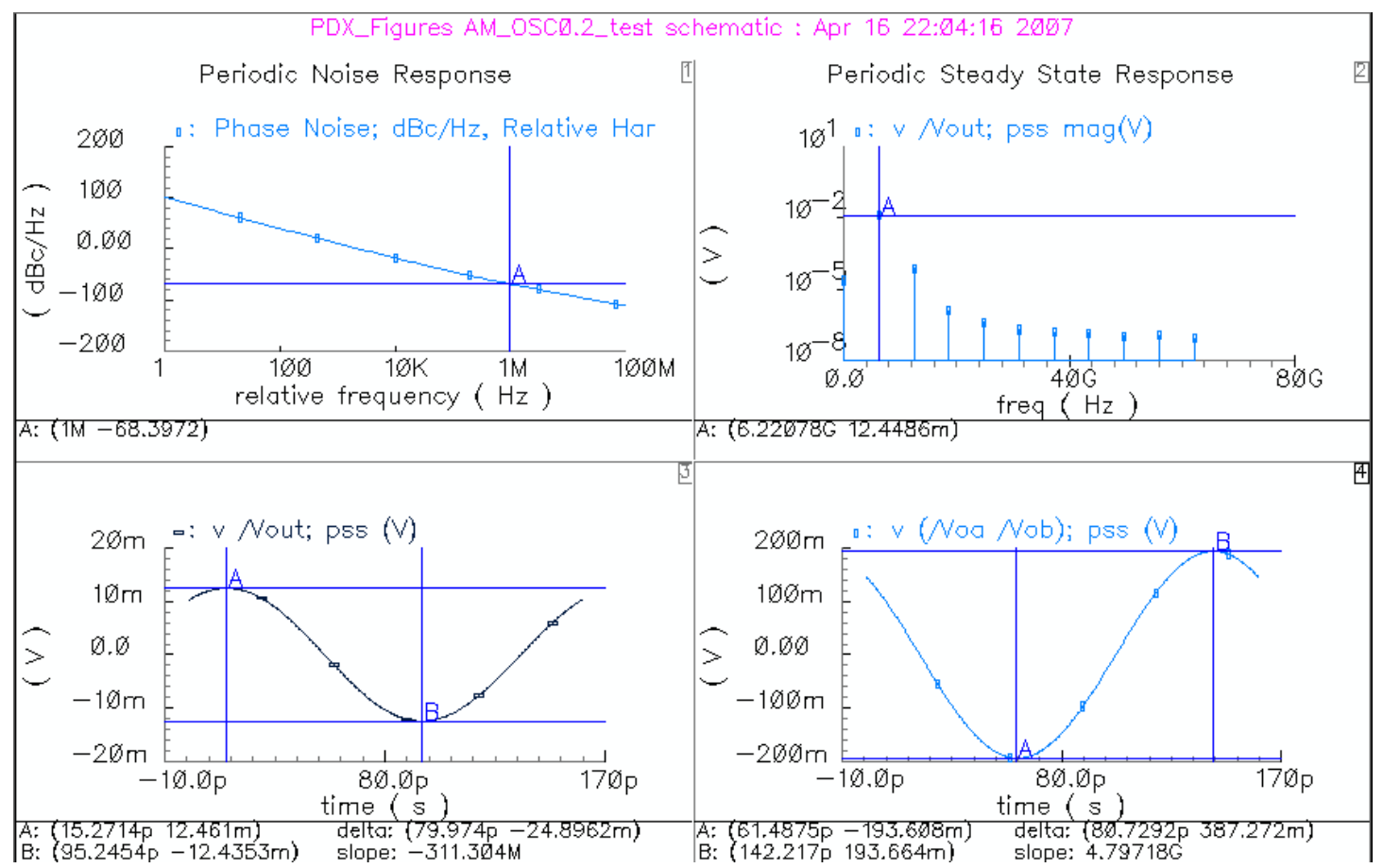

Figure 5-18 Simulation results of the fabricated 5-GHz oscillator with output buffer and load.

The signal amplitude on the $50-\Omega$ load is $12.45 \mathrm{mV}$, corresponding to -25.1 $\mathrm{dBm}$. The output-buffer gain is calculated to be $-23.8 \mathrm{~dB}$, close to the simulation result of $-24.8 \mathrm{~dB}$ at $6.22 \mathrm{GHz}$. The phase noise is $-68.4 \mathrm{dBc} / \mathrm{Hz}$ at $1 \mathrm{MHz}$ offset, 
about $1.8 \mathrm{~dB}$ better than the measured result. We note that a minor design flaw in the fabricated oscillator degraded the phase noise by about $4 \mathrm{~dB}$, as shown in Figs. 5-19 and 5-20.

N13, N1, N4 and N7 in Fig. 5-19 are all biasing transistors, and they do not affect the active-inductor oscillator's frequency directly. Hence they do not have to be minimum-length devices. They are minimum length $(0.2 \mu \mathrm{m})$ in the fabricated oscillator, but have tripled length $(0.6 \mu \mathrm{m})$ and widths in the improved circuit in Fig. 4-8. The phase noise of the latter is lower by $4.1 \mathrm{~dB}$, because these transistors contribute less flicker noise due to the increased gate area. The frequency is reduced by about $6 \%$ due to the use of $0.6-\mu \mathrm{m}$ devices.

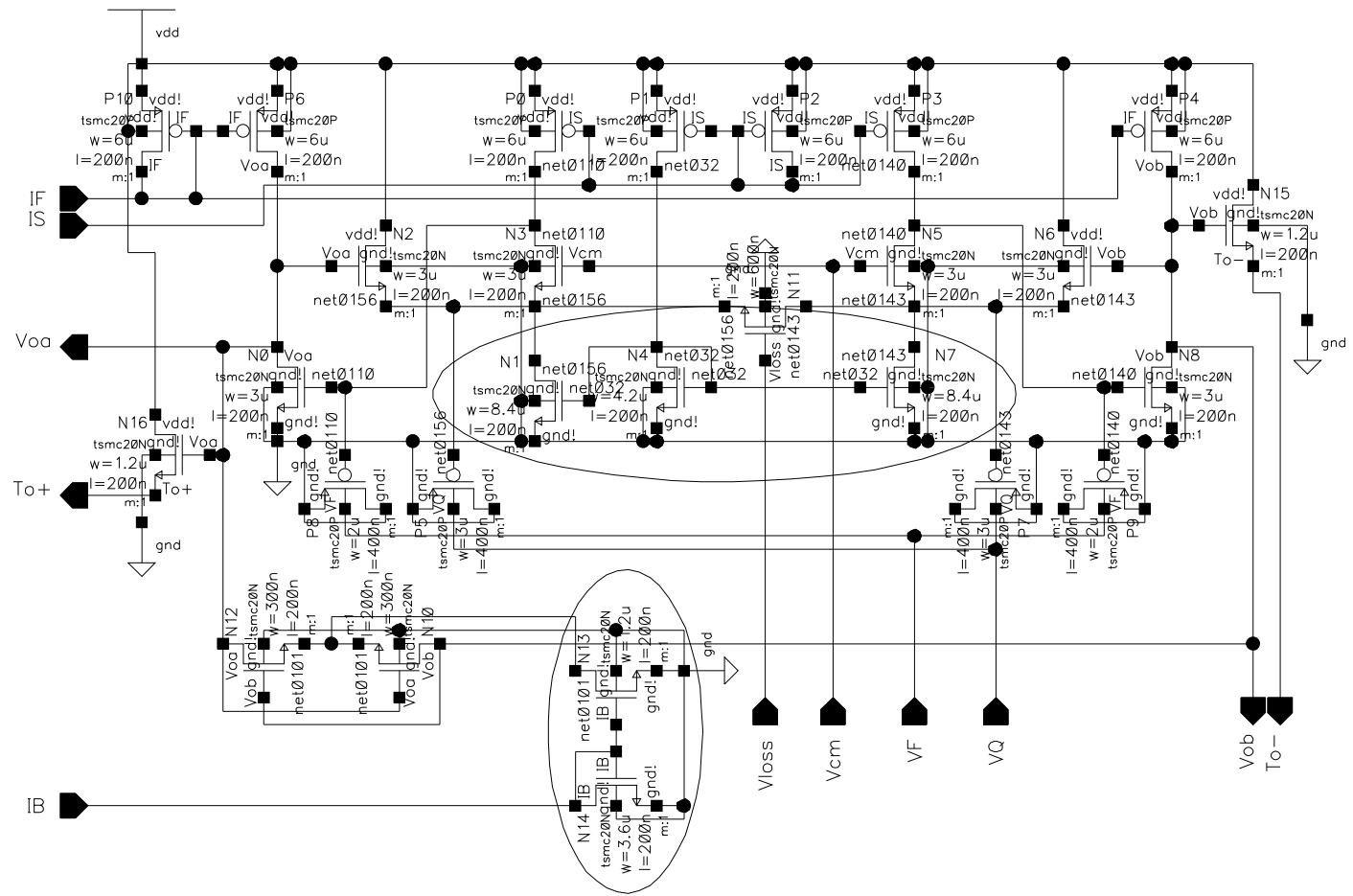

Figure 5-19 A minor design flaw in the fabricated oscillator, shown by the circle. 


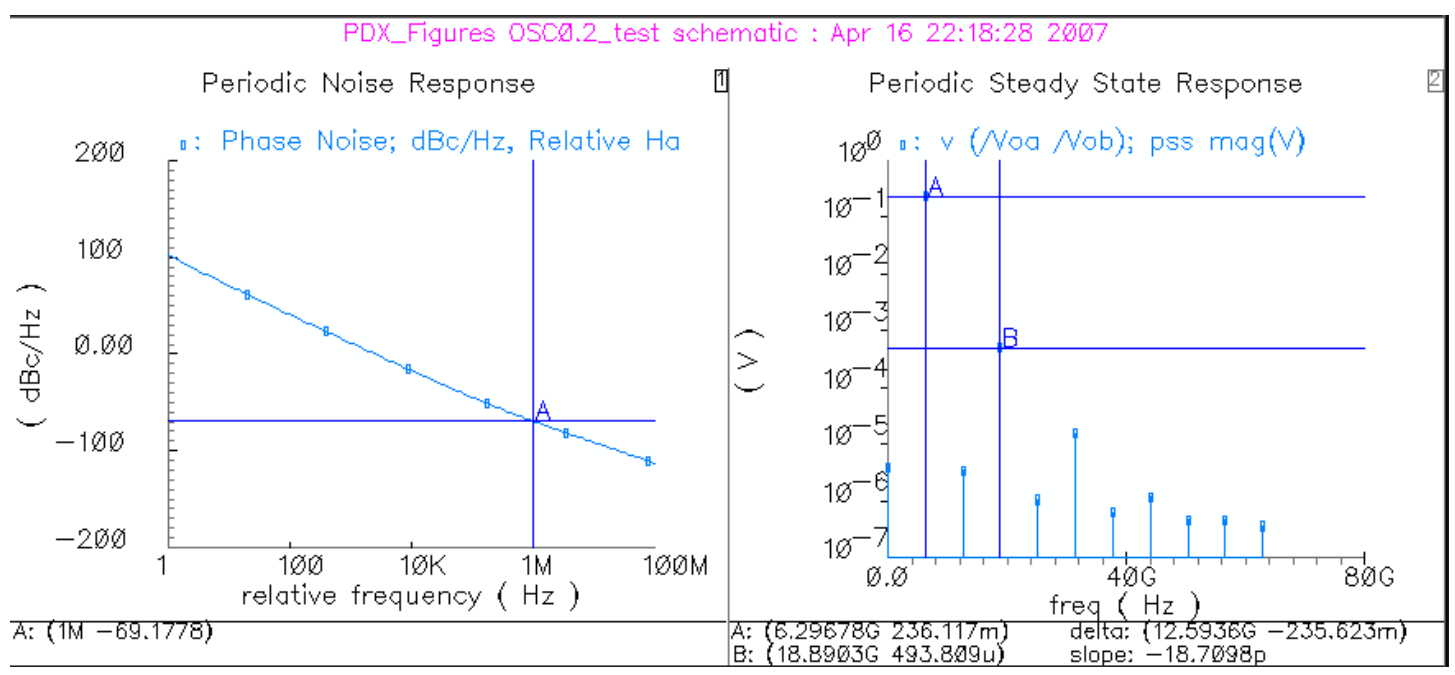

Figure 5-20 The simulated phase noise of the fabricated OSC 0.2 core without output buffer is $4.1 \mathrm{~dB}$ worse than the example shown in Fig. 4-8 due to the smaller $L$ in the tail biasing FET.

\subsubsection{The 2.6-GHz Active Inductor Oscillator (OSC0.4, $L=0.4 \mu \mathrm{m})$}

Figure 5-21 shows the output spectrum of the 2.6-GHz oscillator. The measured frequency is $2.59 \mathrm{GHz}$, and the measured phase noise is $-73.2 \mathrm{dBc} / \mathrm{Hz}$ at $1 \mathrm{MHz}$ offset, as shown in Fig. 5-22. The simulated results are shown in Fig. 5-23. The 3.1$\mathrm{dB}$ phase noise difference between simulation and measurement can be explained by the difference in their $f_{0}$ s. Per Eq. (4.13), the phase noise is proportional to $f_{0}$. Thus if $f_{0}$ increases from $2.363 \mathrm{GHz}$ to $3.036 \mathrm{GHz}$ we should see an increase of phase noise by $20 * \log (3.036 / 2.363)=2.2 \mathrm{~dB}$. 


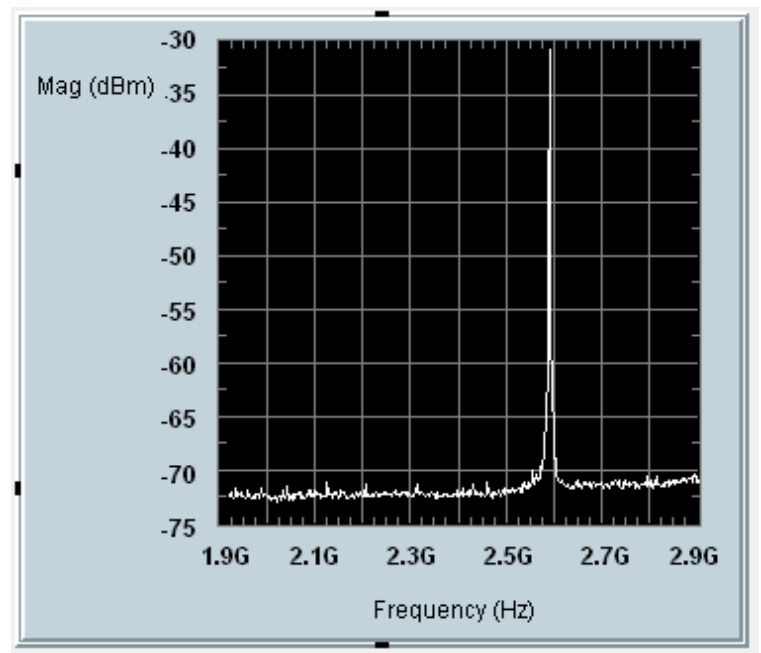

(a)

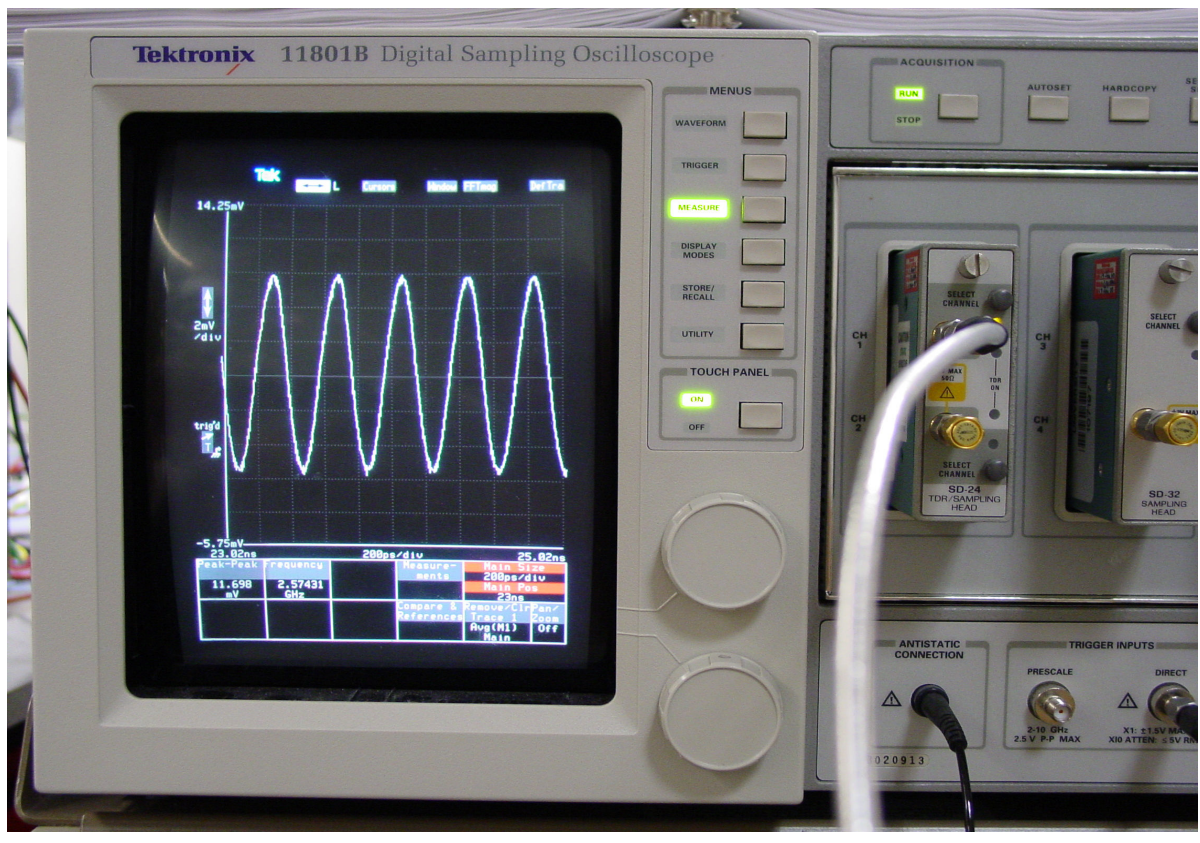

(b)

Figure 5-21 2.6-GHz-oscillator lab results. (a) The output spectrum of the 2.6-GHz oscillator (with 50$\Omega$ output driver), the center frequency is $f_{0}=2.589 \mathrm{GHz}$, the magnitude is $-30.79 \mathrm{dBm}$, corresponding to a peak voltage of $130 \mathrm{mV}$ at the circuit core. (b) Output signal of the $2.6-\mathrm{GHz}$ oscillator displayed on a Tektronix 11801B high-speed digital sampling oscilloscope. The measured signal has a peak-to-peak amplitude of $11.698 \mathrm{mV}$ at a frequency of $2.574 \mathrm{GHz}$, corresponding approximately to a peak voltage of $186 \mathrm{mV}$ at the circuit core when the power splitter and cable attenuation of $7 \mathrm{~dB}$ is deducted. 


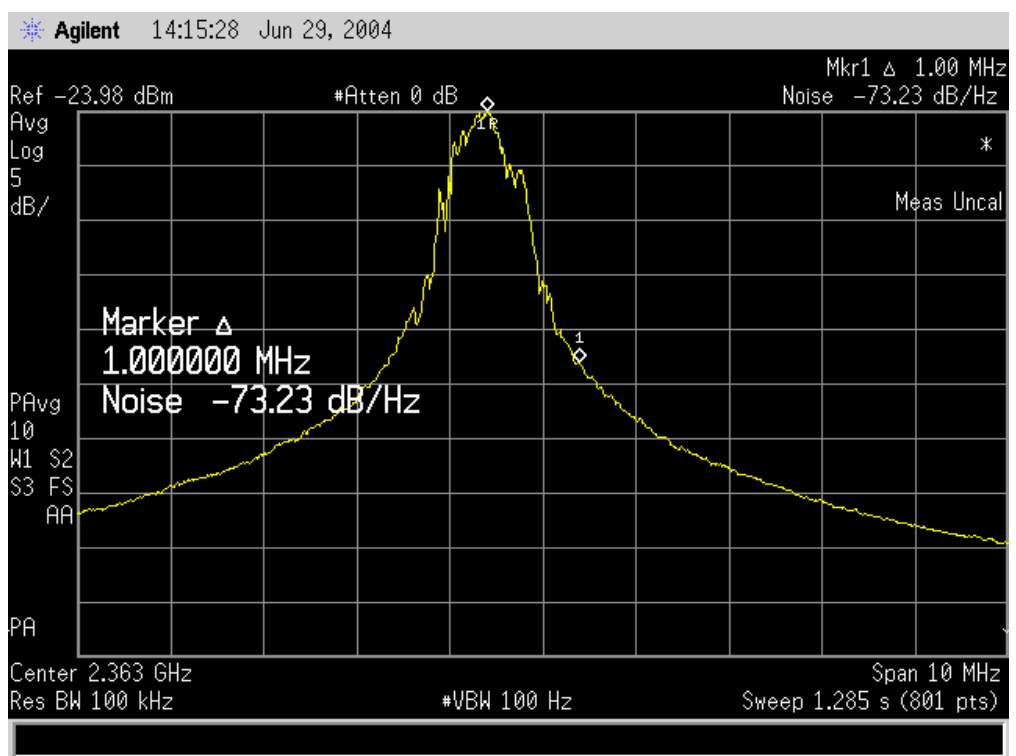

Figure 5-22 Measured phase noise of the 2.6-GHz oscillator (OSC0.4, $L=0.4 \mu \mathrm{m})$.

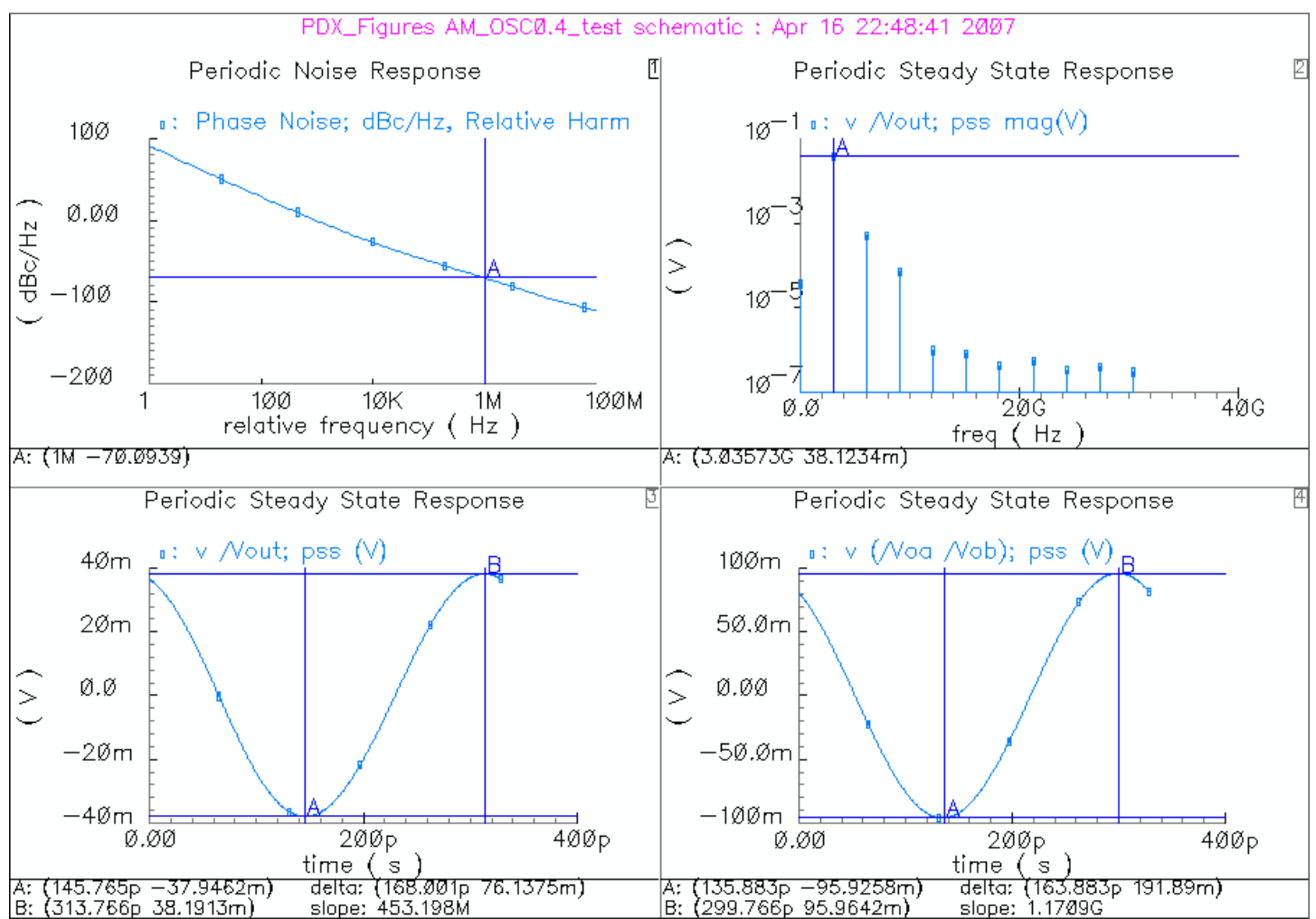

Figure 5-23 Simulation results of the fabricated 2.6-GHz oscillator with output buffer and load. 


\subsubsection{The High- $Q$ Active-Inductor Bandpass Filters}

Figure 5-24 shows the frequency profiles of the high- $Q$ bandpass filters, for $L=0.2$ $\mu \mathrm{m}$ and $L=0.4 \mu \mathrm{m}$, respectively. Figure 5-25 shows the $f_{0}$-variation when $Q$ is tuned via the varactor voltage $V_{\mathrm{Q}} . V_{\mathrm{Q}}$ changes $C_{2}$ in Fig. 2-28, and due to $2^{\text {nd }}$-order effects, changes $f_{0}$ by a small amount. Since the bandpass filter's $Q$ is the active inductor $Q_{\mathrm{R}}$ modified by the extra parasitic elements from the input $G_{\mathrm{M}}$ and output buffer, the $Q_{\mathrm{R}}$ tuning characteristic of an active inductor will be the same (of course, we cannot measure $Q_{\mathrm{R}}$ directly).

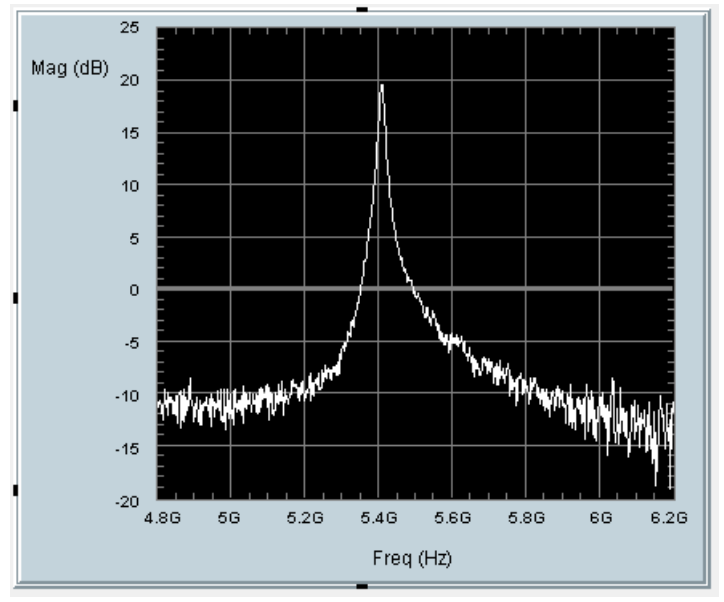

(a)

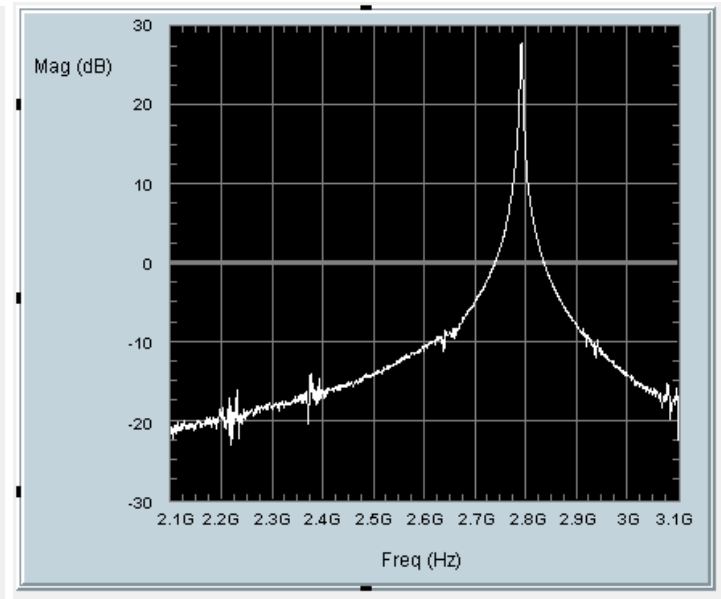

(b)

Figure 5-24 Measured frequency responses of the bandpass filters (S21). (a) $L_{\text {gate }}=0.2 \mu \mathrm{m}, f_{0}=5.4$ $\mathrm{GHz}, Q=365$; (b) $L_{\text {gate }}=0.4 \mu \mathrm{m}, f_{0}=2.79 \mathrm{GHz}, Q=661$. The midband gain is set at 20 to 30 $\mathrm{dB}$ for high SNR and can be adjusted via $I_{\mathrm{B}}$. 


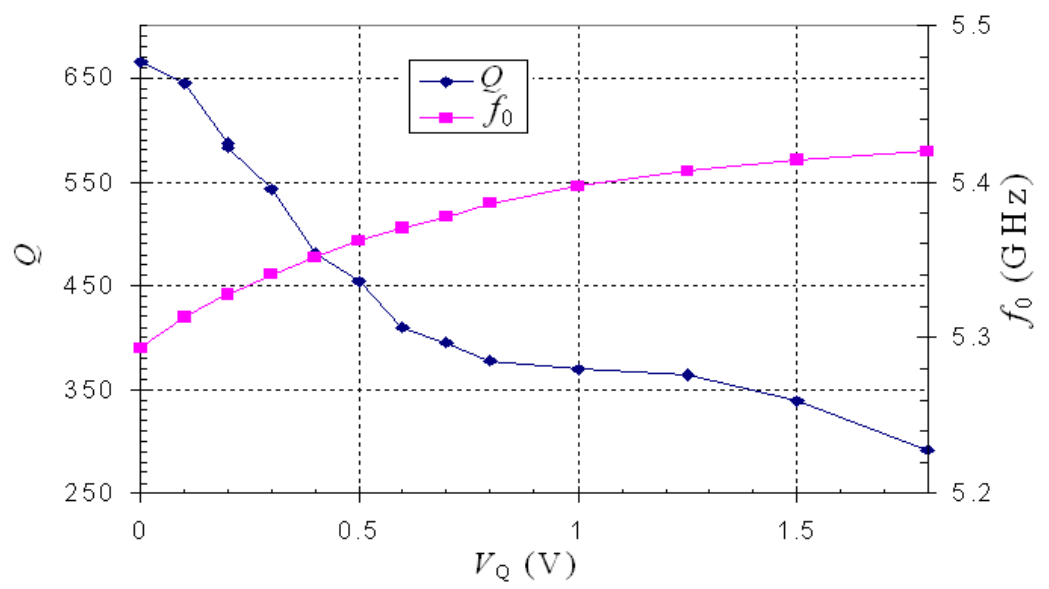

Figure 5-25 Measured $Q$-tuning characteristic of the 0.2- $\mu \mathrm{m}$ gate-length bandpass filter vs. the varactor voltage $V_{\mathrm{Q}}$ for $292 \leq Q \leq 665$. Over this range, $f_{0}$ decreases from $5.42 \mathrm{GHz}$ to $5.3 \mathrm{GHz}$ (a $2 \%$ change). Note that $Q$ can be tuned over different ranges depending on the baseline $Q$ as set by the choice of biases. Here, the baseline $Q$ was set to 410 at $V_{\mathrm{Q}}=0.6 \mathrm{~V}$ by choosing $V_{\mathrm{CM}}=1.29 \mathrm{~V}$ with all other biasing conditions given in Fig. 2-29.

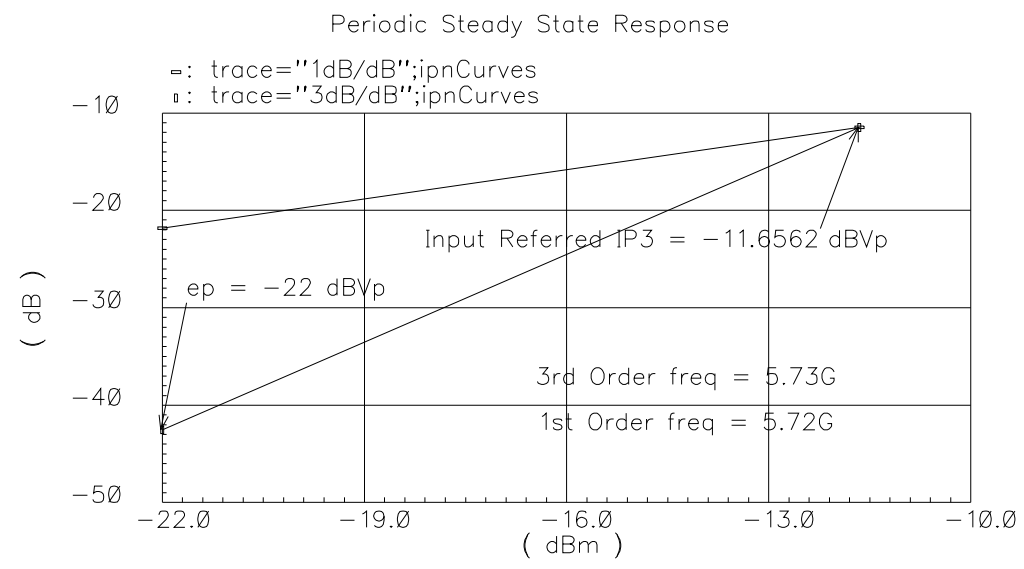

Figure 5-26 Simulation results of IIP3, which is $0.523 \mathrm{~V}_{\mathrm{pp}}$, differentially.

The simulated input-referred $3^{\text {rd }}$-order intercept point (IIP3) is $0.523 V_{\mathrm{pp}}$, as shown in Fig. 5-26. The simulated 1-dB compression point is shown in Fig. 5-27. The simulated noise figure is shown in Fig. 5-28. 


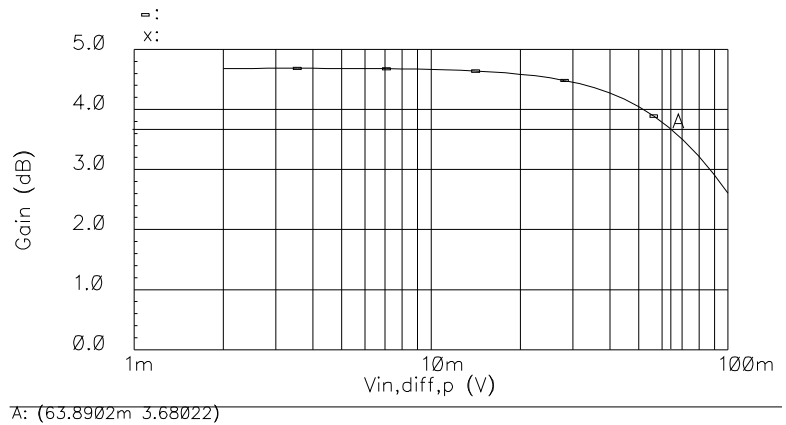

Figure 5-27 Simulation results for deriving the input 1-dB compression point, which is $0.128 \mathrm{Vpp}$, differentially.

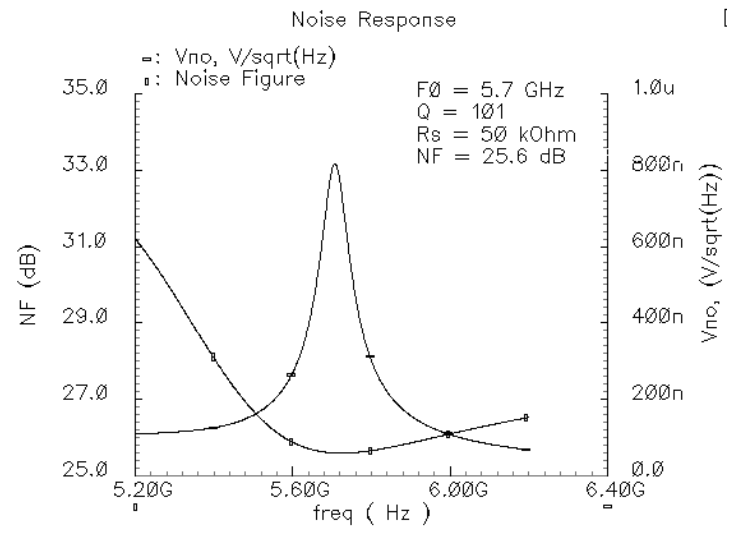

Figure 5-28 Simulated Noise Figure $\left(R_{\mathrm{S}}=50 \mathrm{k} \Omega\right)$ and equivalent output noise.

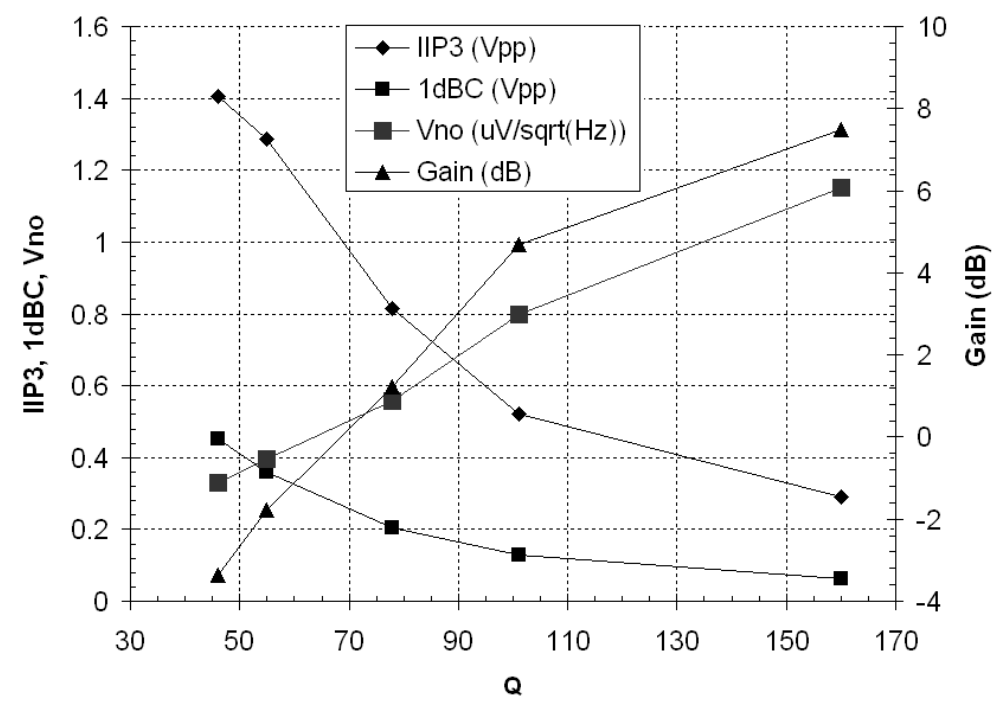

Figure 5-29 Plot of IIP3, input 1-dB compression point, and equivalent output noise.

A higher $Q_{\mathrm{R}}$ is associated with an approximately proportional higher shunt resistor $R_{\mathrm{p}}$ in the active inductor per Eq. (2.62), and consequently, the midband gain is 
higher. However, this means that the input signal and active-inductor internal noise current will both be amplified more; consequently, IIP3 and 1-dB compression point will reduce and the output noise voltage will be higher. This tradeoff is shown in Fig. $5-29$.

The performance of the 5.4-GHz active-inductor filter is compared with two other recent designs in Table 5-1. 
Table 5-1 Performance Summary of the Active-Inductor Filter and Comparison

\begin{tabular}{|c|c|c|c|}
\hline Specifications & This Work & Ref. [45] & Ref. [8] \\
\hline Technology & $0.20-\mu \mathrm{m}$ CMOS & $0.35-\mu \mathrm{m}$ CMOS & $\begin{array}{c}\text { BJT, } 0.35-\mu m \text { SiGe } \\
\text { BiCMOS }\end{array}$ \\
\hline Filter order & 2 & 2 & 2 \\
\hline Layout area & $26.6 \mu \mathrm{m} \times 30 \mu \mathrm{m}$ & $200 \mu \mathrm{m} \times 140 \mu \mathrm{m}$ & $<200 \mu \mathrm{m} \times 200 \mu \mathrm{m}$ \\
\hline$V_{\mathrm{DD}}$ & 1.8 & 2.7 & 3.3 \\
\hline$I_{\mathrm{DD}}$ & $2.44 \mathrm{~mA}$ & $17 \mathrm{~mA}, Q$ dependent & - \\
\hline Power & $4.4 \mathrm{~mW}$ & $45.9 \mathrm{~mW}$ & $<25.2 \mathrm{~mW}$ \\
\hline$f_{0}$ & $5.4 \mathrm{GHz}$ & $0.9 \mathrm{GHz}$ & $6.5,8.3$, and $10 \mathrm{GHz}$ \\
\hline$f_{0}$ tuning range & $3.34 \mathrm{GHz}$ to $5.72 \mathrm{GHz}$ & 0.4 to $1.1 \mathrm{GHz}$ & 6.5 to $10 \mathrm{GHz}$ \\
\hline$Q$ tuning range & 2 to 665 ( $\infty$ possible) & 2 to 80 & 2 to $38^{1}$ \\
\hline$I I P 3$ & $\begin{array}{c}0.523 \mathrm{~V}_{\mathrm{PP}} \text { or }-1.65 \mathrm{dBm} \\
\text { from } 50 \Omega^{1}, 5.7 \mathrm{GHz}, 4.7 \\
\mathrm{~dB} \text { midband gain, } Q=101\end{array}$ & $\begin{array}{c}-15 \mathrm{dBm}\left(0.112 \mathrm{~V}_{\mathrm{PP}}\right), \\
0.9 \mathrm{GHz}, Q=40 \text {, gain } \\
\text { unknown }\end{array}$ & $\begin{array}{c}-26 \mathrm{dBm}^{1}\left(0.032 \mathrm{~V}_{\mathrm{PP}}\right), \\
8.5 \mathrm{GHz}, 15.1 \mathrm{~dB} \\
\text { gain, } Q \text { unknown }\end{array}$ \\
\hline $\begin{array}{l}\text { 1-dB input } \\
\text { compression }\end{array}$ & $\begin{array}{c}0.128 \mathrm{~V}_{\mathrm{PP}} \text { or }-13.9 \mathrm{dBm} \\
\text { from } 50 \Omega^{1}, 5.7 \mathrm{GHz}, 4.7 \\
\mathrm{~dB} \text { midband gain, } Q=101\end{array}$ & - & $\begin{array}{c}-34 \mathrm{dBm}^{1}(12.6 \\
\left.\mathrm{mV}_{\mathrm{PP}}\right), 8.5 \mathrm{GHz}, 15.1 \\
\mathrm{~dB} \text { gain, } Q \text { unknown }\end{array}$ \\
\hline Output Noise & $\begin{array}{c}0.8 \mu \mathrm{V} / \mathrm{Hz}^{1 / 21}, 5.7 \mathrm{GHz} \\
4.7 \mathrm{~dB} \text { midband gain, } Q= \\
101\end{array}$ & $-147 \mathrm{dBm} / \mathrm{Hz}$ & - \\
\hline Noise Figure & $\begin{array}{c}25.6 \mathrm{~dB} \text { at } 5.7 \mathrm{GHz}^{1}, R_{\mathrm{S}}= \\
50 \mathrm{k} \Omega \text {, others same as } \\
\text { above }\end{array}$ & - & $\begin{array}{c}8.8-10.4 \mathrm{~dB}^{1}, R_{\mathrm{S}} \\
\text { unknown }\end{array}$ \\
\hline $\begin{array}{l}f_{0} \text { statistics, } \sigma, L_{\text {gate }}= \\
0.2 / 0.4 \mu \mathrm{m}, \text { lot of } 40\end{array}$ & $1.78 \% / 0.72 \% f_{0}$ & - & - \\
\hline $\begin{array}{c}f_{0} \text { statistics, range, } L_{\text {gate }} \\
=0.2 / 0.4 \mu \mathrm{m} \text {, lot of } \\
40\end{array}$ & $\pm 4.2 \% / \pm 1.65 \% f_{0}$ & - & - \\
\hline
\end{tabular}

Simulation results.

\subsubsection{Frequency Tuning of the Active Inductor}

When used as a resonator in oscillators and bandpass filters, the active inductor's $f_{\mathrm{R}}$ and $Q_{\mathrm{R}}$ need to be tuned to desired values. We have seen the filter $Q$ tuning in Fig. 525. Since the bandpass filter's $Q$ is the active inductor $Q_{\mathrm{R}}$ modified by the extra parasitic elements from the input $G_{\mathrm{M}}$ and output buffer, the $Q_{\mathrm{R}}$ tuning characteristic of an active inductor will be the same.

Active inductor's $f_{\mathrm{R}}$ can be tuned via the biasing currents $I_{\mathrm{F}}$ and $I_{\mathrm{S}}$ as well as the varactor control voltage $V_{\mathrm{F}}$. Current tuning can be used as coarse tuning or as a design 
measure, and $V_{\mathrm{F}}$ tuning can be used as fine tuning. A tuning example is shown in Fig. 5-30. The results are measured from an active-inductor oscillator. Similarly, since the oscillator's $f_{0}$ is the active inductor's $f_{\mathrm{R}}$ modified by the extra parasitic elements from the cross-connected differential pair and output buffer, the $f_{0}$ tuning characteristic in Fig. 5-30 represents the active inductor $f_{\mathrm{R}}$ tuning characteristic as well.

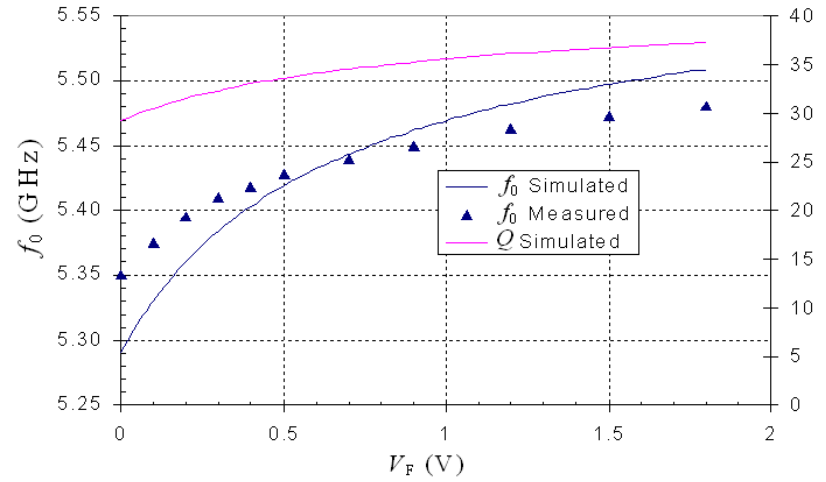

(a)

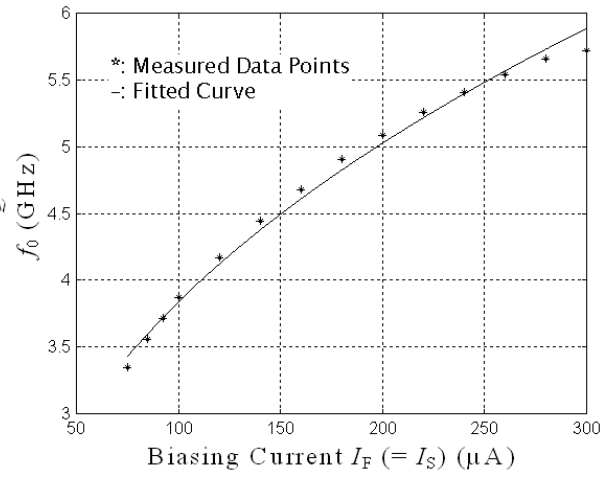

(b)

Figure 5-30 Tuning the filter center frequency $\left(L_{\text {gate }}=0.2 \mu \mathrm{m}\right)$ : (a) frequency-tuning via the varactor voltage $V_{\mathrm{F}} ;(\mathrm{b})$ frequency-tuning via biasing currents $I_{\mathrm{F}}$ and $I_{\mathrm{S}}$.

In the example, for $0 \mathrm{~V} \leq V_{\mathrm{F}} \leq 1.8 \mathrm{~V}, f_{0}$ is measured in the range $5.35 \mathrm{GHz} \leq f_{0} \leq$ $5.48 \mathrm{GHz}$; the simulated $f_{0}$ changes from $5.29 \mathrm{GHz}$ to $5.51 \mathrm{GHz}$. For this range of $V_{\mathrm{F}}$ the simulated $Q$ changes from 29 to 37 , with the baseline $Q$ set at 34 by $V_{\mathrm{CM}}=1.31 \mathrm{~V}$. Note that $Q$ will be more sensitive to $V_{\mathrm{F}}$ if the baseline $Q$ is high. The difference between simulation and measurement of $f_{0}$ is mainly due to the inaccuracies in extracting the parasitic capacitors. When the biasing currents $I_{\mathrm{F}}$ and $I_{\mathrm{S}}$ are varied from $75 \mu \mathrm{A}$ to $300 \mu \mathrm{A}, f_{0}$ changes from $3.34 \mathrm{GHz}$ to $5.72 \mathrm{GHz}$. $Q_{\mathrm{R}}$ varies over a large range when varying $I_{\mathrm{F}}$ and must be reset by iterative circuit biasing. For this reason $f_{0^{-}}$ control via $I_{\mathrm{F}}$ is intended to be a design measure rather than an on-chip tuning method.

Of course, another design measure for $f_{0}$ is the gate length $L$ of the primary FETs in the active inductor, per Eq. (4.17). 


\subsubsection{Measured Frequency Statistics}

A total of 40 chips are available for testing. This permits us to derive some statistical information, which reflects to some extent the process variation and the active inductor's sensitivities to it. Figure 5-31 shows the frequency histogram of the oscillators. The distribution is approximately Gaussian.

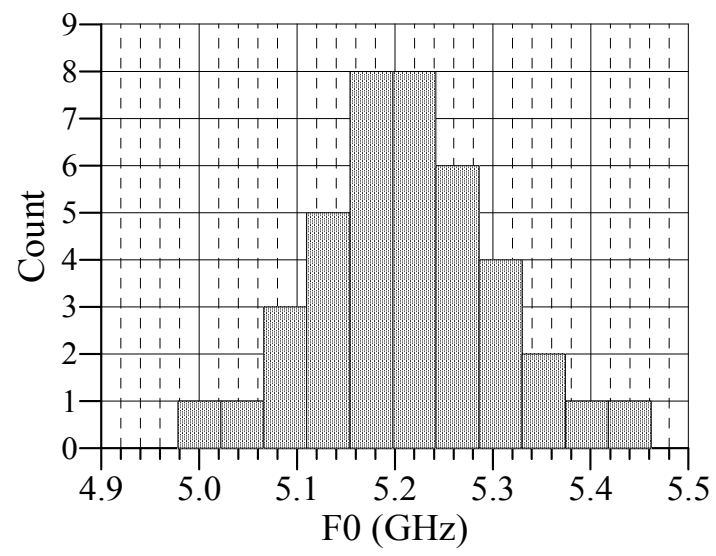

(a)

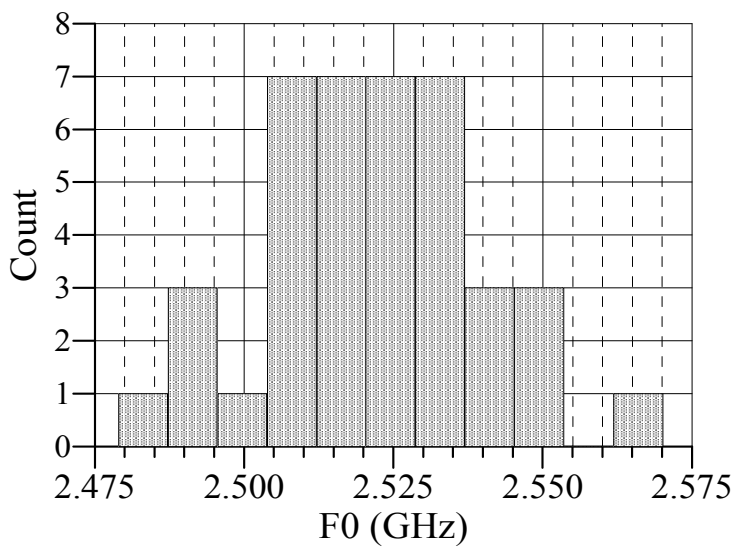

(b)

Figure 5-31 Histograms of oscillator $f_{0}$ at nominal biasing. (a) 5-GHz oscillator, $L_{\text {gate }}=0.2 \mu \mathrm{m}$, OSC0.2; (b) $2.5-\mathrm{GHz}$ oscillator, $L_{\text {gate }}=0.4 \mu \mathrm{m}$, OSC0.4.

Table 5-2 shows the statistics results. Each oscillator module is tested on all 40 chips at two biasing levels, nominal $\left(I_{\mathrm{F}}=I_{\mathrm{S}}=250 \mu \mathrm{A}\right)$ and higher $\left(I_{\mathrm{F}}=I_{\mathrm{S}}=280 \mu \mathrm{A}\right)$. We may therefore calculate the statistics of the $\Delta f_{0}$ between the two biasing settings. The average $f_{0}$ of the higher- $Q$ modules is always lower than that of regular- $Q$ modules because of their larger $Q$-tuning varactor used to increase $C_{2}$ in Fig. 2-28, which increases the total module capacitance. The standard deviation of $f_{0}$ for the 2.5$\mathrm{GHz}$ modules is only about $20 \%$ that of the $5-\mathrm{GHz}$ modules, because their larger transistor $W$ and $L$ translate into a smaller process-variation percentage.

We expect the same $f_{0}$ statistics for high- $Q$ bandpass filters since the filters and oscillators are designed using the same active-inductor core. Table 5-3 summarizes the 
measured results on the oscillators and filters.

Table 5-2 Frequency $\left(F_{0}\right)$ statistics of the oscillators.

\begin{tabular}{|c|c|c|c|c|c|}
\hline & & OSC0.2 & $\overline{\mathrm{OSC} 0.2 \mathrm{HQ}}$ & OSC0.4 & $\overline{\mathrm{OSC} 0.4 \mathrm{HQ}}$ \\
\hline \multirow{6}{*}{$\begin{array}{l}\text { Nominal } \\
\text { Biasing } \\
\left(I_{\mathrm{F}}=I_{\mathrm{S}}\right. \\
=250 \\
\mu \mathrm{A})\end{array}$} & Average (GHz) & 5.216 & 4.938 & 2.521 & 2.463 \\
\hline & Std Dev (MHz) & 92.67 & 101.5 & 18.05 & 21.33 \\
\hline & $\operatorname{Min}(\mathrm{GHz})$ & 5.0 & 4.73 & 2.483 & 2.409 \\
\hline & $\operatorname{Max}(\mathrm{GHz})$ & 5.44 & 5.128 & 2.566 & 2.5 \\
\hline & Range (MHz) & 440 & 398 & 82.9 & 90.6 \\
\hline & Range/Std Dev & 4.748 & 3.921 & 4.592 & 4.247 \\
\hline \multirow{6}{*}{$\begin{array}{l}\text { Higher } \\
\text { Biasing } \\
\left(I_{\mathrm{F}}=I_{\mathrm{S}}\right. \\
=280 \\
\mu \mathrm{A})\end{array}$} & Average (GHz) & 5.289 & 5.015 & 2.567 & 2.507 \\
\hline & Std Dev (MHz) & 88.83 & 97.47 & 17.96 & 21.59 \\
\hline & $\operatorname{Min}(\mathrm{GHz})$ & 5.075 & 4.818 & 2.53 & 2.454 \\
\hline & $\operatorname{Max}(\mathrm{GHz})$ & 5.503 & 5.2 & 2.611 & 2.544 \\
\hline & Range (MHz) & 428 & 382 & 81.5 & 90.4 \\
\hline & Range/Std Dev & 4.818 & 3.919 & 4.537 & 4.188 \\
\hline \multirow{6}{*}{$\begin{array}{l}\text { Delta } f_{0} \\
\text { between } \\
\text { the two } \\
\text { biasings }\end{array}$} & $\begin{array}{l}\text { Average } \\
(\mathrm{MHz})\end{array}$ & 73.81 & 77.41 & 45.5 & 44.15 \\
\hline & Std Dev (MHz) & 5.085 & 5.882 & 1.234 & 1.488 \\
\hline & $\operatorname{Min}(\mathrm{MHz})$ & 63.0 & 65.0 & 43.0 & 42.0 \\
\hline & $\operatorname{Max}(\mathrm{MHz})$ & 85.0 & 93.0 & 48.4 & 46.4 \\
\hline & Range (MHz) & 22.0 & 28.0 & 5.4 & 4.2 \\
\hline & Range/Std Dev & 4.327 & 4.76 & 4.377 & 2.822 \\
\hline
\end{tabular}

\subsubsection{Summary of Measured Results}

Table 5-3 Summary of measured results.

\begin{tabular}{|c|c|c|c|c|c|c|c|c|}
\hline & \multicolumn{2}{|c|}{ OSC0.2 } & \multicolumn{2}{c|}{ OSC0.4 } & \multicolumn{2}{c|}{ BP0.2 } & \multicolumn{2}{c|}{ BP0.4 } \\
\hline & Measure & Simul'd & Measure & Simul'd & Measure & Simul'd & Measure & Simul'd \\
\hline$L_{\text {gate }(\mu \mathrm{m})}$ & 0.2 & 0.2 & 0.4 & 0.4 & 0.2 & 0.2 & 0.4 & 0.4 \\
\hline$f_{0}(\mathrm{GHz})$ & 5.645 & 6.221 & 2.6 & 3.036 & 5.4 & $6.4^{2}$ & 2.8 & - \\
\hline $\begin{array}{c}\text { Peak Amp } \\
(\mathrm{mV})\end{array}$ & 134 & 194 & 130 & 96 & - & - & - & - \\
\hline $\begin{array}{c}\text { Phase } \\
\text { Noise @ } 1 \\
\text { MHz } \\
(\mathrm{dBc} / \mathrm{Hz})\end{array}$ & $-66.6^{1}$ & $-68.4^{1}$ & $-73.2^{1}$ & $-70.1^{1}$ & - & - & & - \\
\hline$Q$ & - & - & - & - & 365 & Varies & 661 & - \\
\hline $\begin{array}{c}f_{0} \mathrm{mean} \\
(\mathrm{GHz})\end{array}$ & 5.216 & - & 2.521 & - & - & - & & - \\
\hline $\begin{array}{c}f_{0} \mathrm{stdev} \\
(\mathrm{MHz})\end{array}$ & 92.67 & - & 18.05 & - & - & - & & - \\
\hline
\end{tabular}

due to a design flaw (see Section 5.6.1), the phase noise is $4 \mathrm{~dB}$ higher than it could be.

${ }^{2}$ Figure 3-7.

\subsection{Results of Active-Inductor Lowpass Filters}

Active-inductor lowpass filters with $L_{\text {gate }}=0.2 \mu \mathrm{m}$ (LP0.2) and $0.4 \mu \mathrm{m}$ (LP0.4) were 
fabricated and measured, with results shown in Figs. 5-32 and 5-33, respectively.

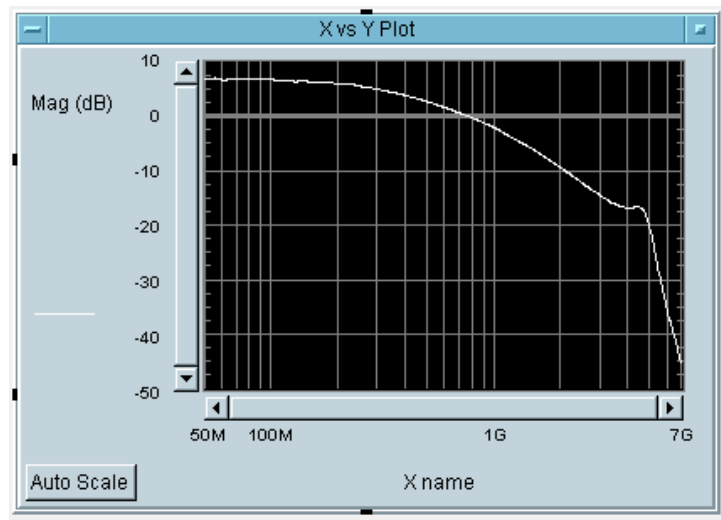

(a)

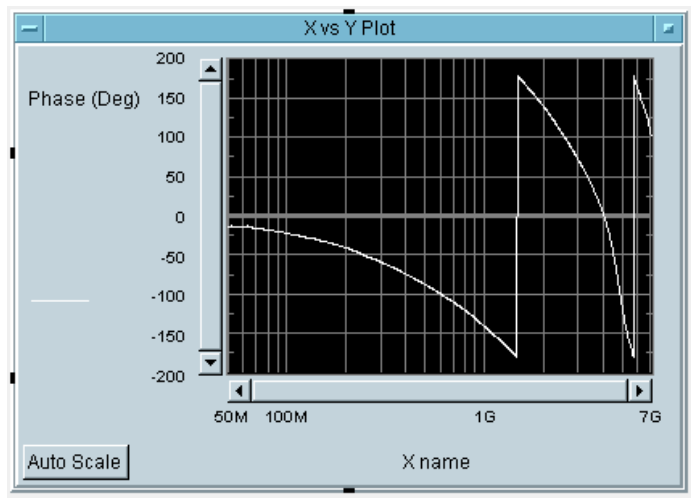

(c)

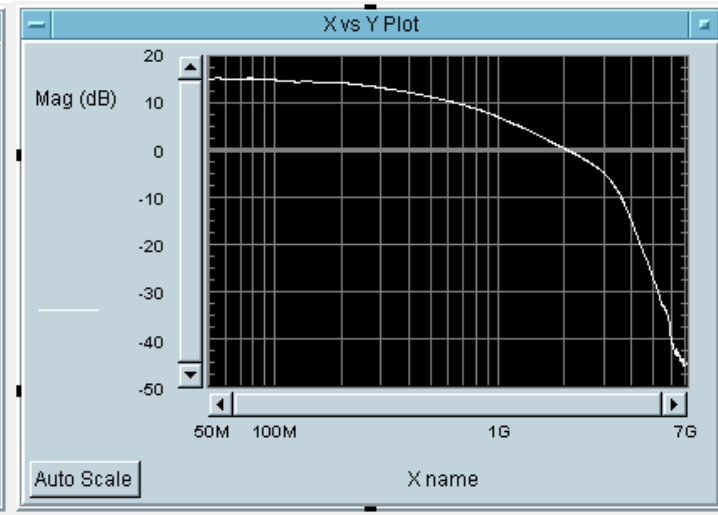

(b)

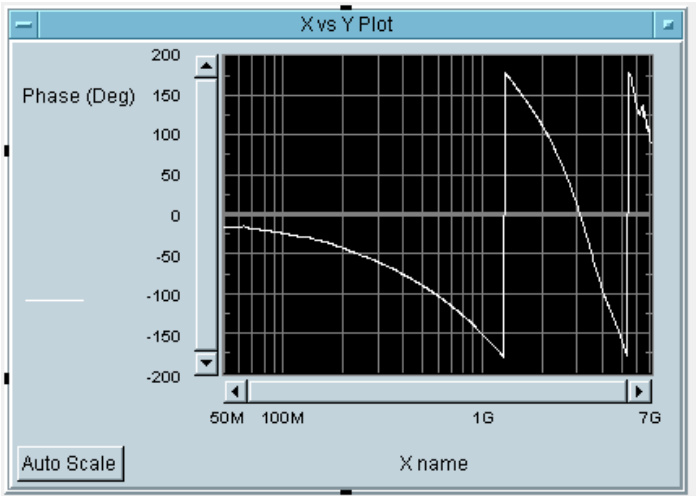

(d)

Figure 5-32 Examples of magnitude and phase responses of the module LP0.2, including the input buffer and the output driver. (a, c) $Q$ is a little too high as shown by the peaking at the passband corner. The pole frequency is about $4.5 \mathrm{GHz}$; (b, d) $Q$ is reduced via the loss resistor, resulting in less peaking. (Data Ref\# LP0.2-C17-VNA-00010 and LP0.2-C17-VNA-00020)

The filters operate as intended, as shown by the frequency profiles. However, since the frequency-sweep range is very wide, from $50 \mathrm{MHz}$ to $7 \mathrm{GHz}$, the frequency response of the microprobe contact and cable assembly is not constant across the frequencies, and distorts both the measured lowpass filter and reference channel gain profiles. Therefore, after subtracting the reference channel to exclude the effects of the input and output buffers, the lowpass filter frequency profile is distorted, as shown in Fig. 5-35, even though the distortion is barely visible in the raw data of the lowpass filter; the reason is, in part, because the distortion is the result of subtracting two large 
numbers. Nevertheless, the measured results confirm that via appropriate connections the active inductor can operate as lowpass filters.

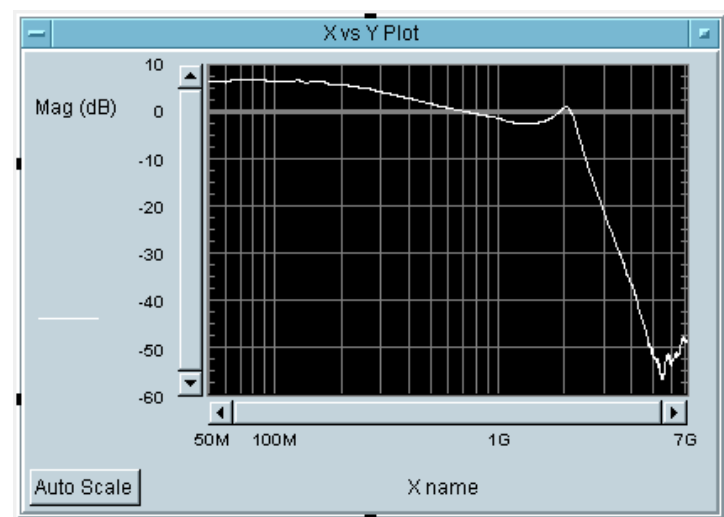

(a)

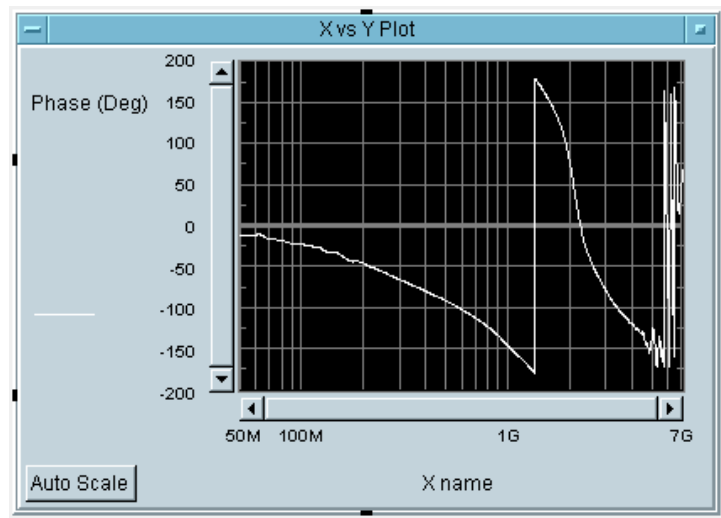

(c)

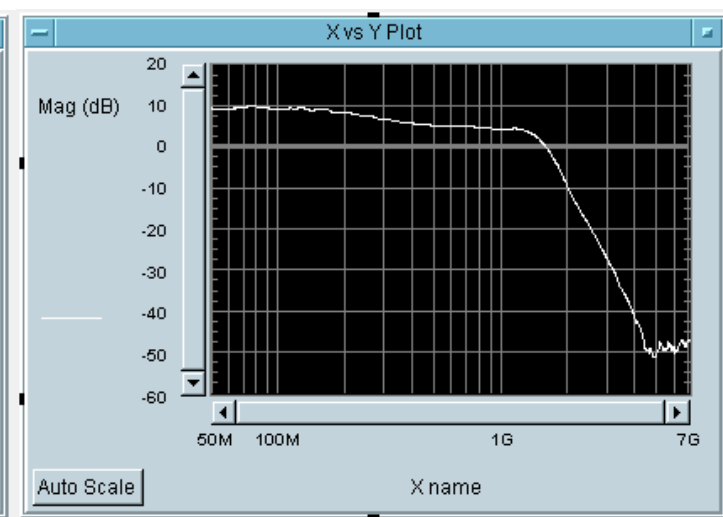

(b)

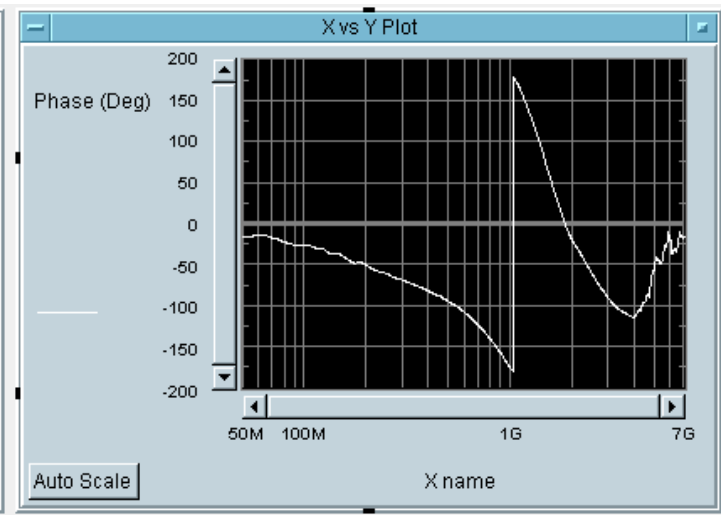

(d)

Figure 5-33 Examples of magnitude and phase responses of the module LP0.4, including the input buffer and the output driver. (a, c) $Q$ is a little high, leading to peaking at the passband corner. The pole frequency is about $2 \mathrm{GHz} ;(\mathrm{b}, \mathrm{d}) Q$ is reduced via the loss resistor, leading to less peaking. (Data Ref\# LP0.4-C17-VNA-00010 and LP0.4-C17-VNA-00020) 


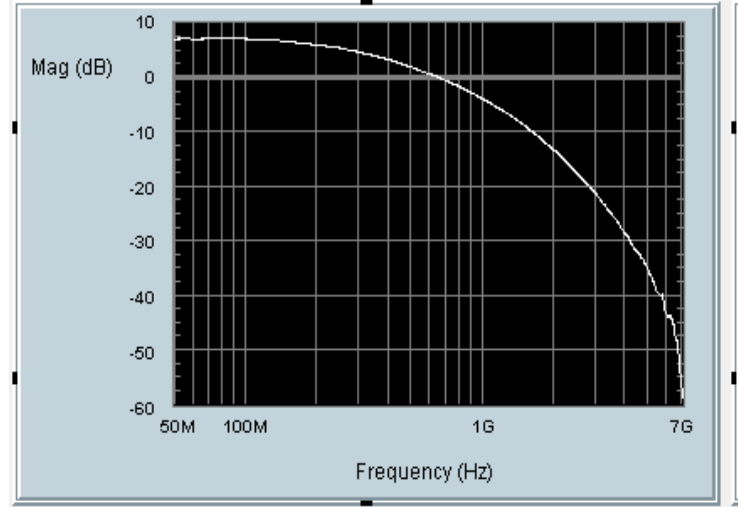

(a)

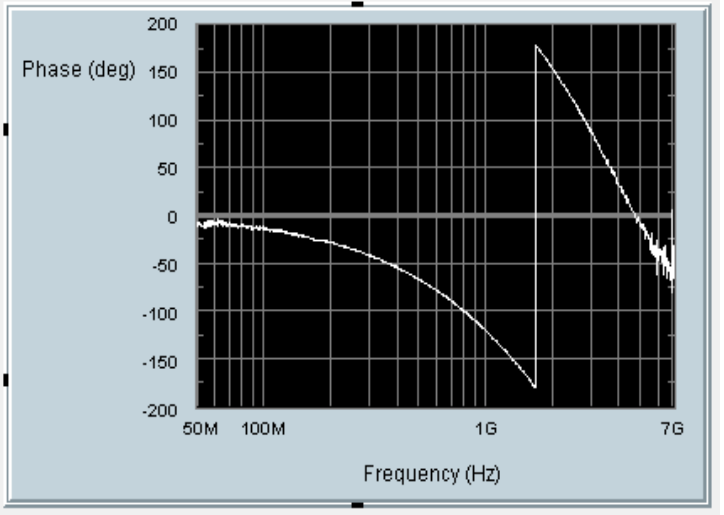

(b)

Figure 5-34 Magnitude and phase responses of the reference channel. (Data Ref\# Ref-C17-VNA-00020, $-00011)$

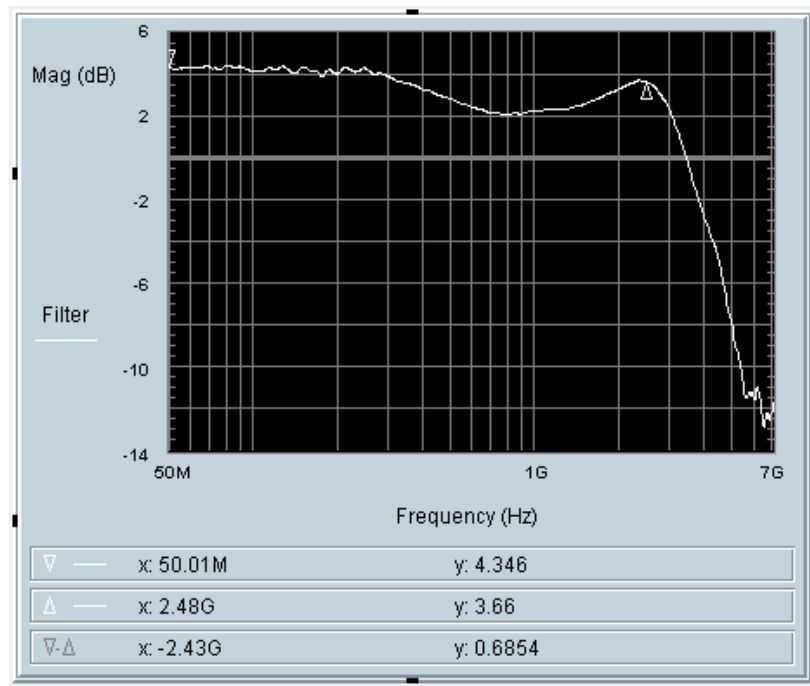

Figure 5-35 0.2- $\mu \mathrm{m}$ lowpass filter frequency response, after subtracting the reference channel. (Data Ref\# LP0.2-C28-VNA-00090 and Ref-C26-VNA-00080)

Different from the bandpass filters, the passband gain of the lowpass filters is approximately 1 , while the bandpass filter can have 20-30 dB gain by choice of the input $G_{\mathrm{M}}$ value. The high-frequency feed-through effect due to parasitic capacitance and substrate, however, is about the same for both the lowpass and bandpass filters, and sets a limit on stopband attenuation. Therefore, the gain difference between passband and stopband is expected to be much smaller for lowpass filters and to limit their use. 


\subsection{Conclusion}

The all-NMOS active inductor circuit obtained in this work was used to design oscillators, bandpass and lowpass filters, for a total of 10 circuit module, including an input-output buffer reference module. The oscillators and filters are fabricated in TSMC's $0.18-\mu \mathrm{m}$ logic CMOS process (LOG018) and the 40 ICs are all tested. All

circuits are functional, with frequencies being about $80-90 \%$ those of the simulation results, due to model and parasitic-capacitance extraction errors. 


\section{CHAPTER 6 \\ CONCLUSION}

The rapid growth of wireless applications in consumer products and industrial systems has generated considerable interest in radio-frequency integrated circuits (RFICs), and a demand for a great variety of different products with emphasis on low cost. The passive on-chip inductor is the staple of wireless communication ICs in order for implementing RF filters and oscillators as well as impedance matching. Passive inductors typically occupy $20-50 \%$ of the total silicon area and require special technologies, such as thicker metal layers or a high-resistivity substrate, resulting in higher cost. Their lack of tunability makes designing a robust product across process corners challenging and requires stringent modeling accuracy. Active inductors, on the other hand, occupy merely $1-10 \%$ the area of passive inductors, and they can be continuously tuned to cover process/temperature variations and to operate over a wide frequency range. When properly designed, they use only transistors and can be fully compatible with standard digital CMOS circuitry on the same die.

The main disadvantages of active inductors are their higher noise, nonlinearity, and power consumption, because translating capacitors into simulated inductors requires multiple-transistor circuitry that has to be biased at high current densities with low-value capacitors to achieve high operating frequencies; linearization schemes usually cannot be used as they generate unavoidable parasitic poles and reduce the frequency range of operation. To date, to the author's knowledge, no active inductors, including this work, can meet the noise and dynamic-range requirements of RF band- 
selection filters or local oscillators in short/long-range wireless communication ICs and consume an acceptable amount of power. However, active inductor circuits have the advantage of saving significant silicon area and may be used in less stringent applications, such as limiting amplifiers and digital clock generators.

The goal of this research was to design and test narrow-band (very-high- $Q$ ) bandpass filters and oscillators operating in several $\mathrm{GHz}$ using active inductors. To achieve this goal, a high-frequency high- $Q$ active-inductor circuit is required. Various active inductors have been proposed in the literature, and some structures have been adopted by industry. However, as discussed in Chapter 2, the majority of these circuits have low $Q$ s due to the presence of a transistor source/emitter terminal at the inductor's primary (input or gyration) node, and/or have biasing difficulties. Therefore, an all-NMOS-signal-path inductor circuit is proposed and experimentally validated in silicon. The circuit has a moderate intrinsic $Q$; but by tuning the parasitic capacitance at an internal node, the output loss of short-channel devices can be partially or totally compensated, and $Q$ can be arbitrarily high. A practical difficulty, though, is that an excessively high $Q$ leads to excessively high circuit sensitivities.

The signal path of the inductor circuit consists of only NFETs, which have higher device mobility than PFETs, hence the circuit can operate at a higher frequency, when compared with one that consists of both PFETs and NFETs in its signal path.

The all-NMOS active inductor was used to design high- $Q$ bandpass filters and oscillators, using TSMC's $0.18-\mu \mathrm{m}$ logic process, and both were fabricated in $0.2-\mu \mathrm{m}$ 
and $0.4-\mu \mathrm{m}$ gate lengths. The highest center frequency was measured to be $5.7 \mathrm{GHz}$ in $0.2-\mu \mathrm{m}$ gate length, and the maximum repeatably measured $Q$ was 665 . The oscillator phase noise was measured to be $-66.6 \mathrm{dBc} / \mathrm{Hz}$ at $1 \mathrm{MHz}$ offset from $5.1-\mathrm{GHz}$ center frequency with 4.4-mW power dissipation. The simulated noise figure for the activeinductor filter is $25.6 \mathrm{~dB}$ at a $50-\mathrm{k} \Omega$ source impedance. The area of the filter is 26.6 $\mu \mathrm{m} \times 30 \mu \mathrm{m}$.

Based on the same gyrator and feedback principle, a circuit labeled all-NMOSII, was developed. It has superior signal fidelity when used as a voltage follower or signal driver, because its gain is unity and constant across process and temperature variations; the input and output dc voltages are the same, limited only by device mismatches.

The all-NMOS active-inductor circuit was granted a US patent, and all-NMOS-II circuit has a US patent pending.

This research proved the possibility of designing very-high- $Q$ active-inductor filters and oscillators at 1-5 GHz using MOSFETs only in standard digital CMOS processes. However, the high level of noise, as determined by the fundamental limits in devices and the requirement of high frequency, will limit their direct use. 


\section{LIST OF REFERENCES:}

1. Behzad Razavi, RF Microelectronics, Prentice Hall, 1998.

2. Steven C. Chan, Kenneth L. Shepard and Phillip J. Restle, "Distributed Differential Oscillators for Global Clock Networks," JSSC, Vol. 41, No. 9, pp. 2083-2093, Sept. 2006.

3. The American Heritage Dictionary of the English Language, $4^{\text {th }}$ Edition, Houghton Mifflin Company, 2000.

4. Sergio Franco, Design with Operational Amplifiers and Analog Integrated Circuits, ${ }^{\text {rd }}$-Ed., McGraw-Hill, New York, 2002.

5. Dandan Li, Y. P. Tsividis, "A Loss-Control Feedback Loop for VCO Indirect Tuning of RF Integrated Filters," IEEE Transactions on Circuits and Systems II, Vol. 47, No. 3, pp. 169-175, March 2000.

6. W. A. Gee, P. E. Allen, "CMOS Integrated Transformer-Feedback QEnhancement LC Bandpass Filter for Wireless Receivers," IEEE Intl. Symp. on Circuits and Systems (ISCAS 2004), Vol. 4, pp. 253-256, May 23-26, 2004.

7. Da-Chiang Chang and Ching-Wen Hsue, "Wide-band Equal-Ripple Filters in Nonuniform Transmission lines," IEEE Trans. On Microwave Theory and Techniques, Vol. 50, No. 4, pp. 1114-19, April 2002.

8. S. Andersson and C. Svensson, "An Active Recursive RF Filter in $0.35 \mu \mathrm{m}$ BiCMOS," Analog Integrated Circuits and Signal Processing, Vol. 44, pp. 213218, 2005.

9. Bernard D. H. Tellegen, "The gyrator, a new electric network element", Philips Res. Rep. 3 (1948) pgs 81-101.

10. Rolf Schaumann, Mac E. Van Valkenburg, "Design of Analog Filters," Oxford University Press, New York, 2001.

11. Peter Ashburn, SiGe Heterojunction Bipolar Transistors, John Wiley \& Sons, West Sussex, England, 2003.

12. Jafar Savoj, Behzad Razavi, High-Speed CMOS Circuits for Optical Receivers, Kluwer Academic Publishers, 2001.

13. Tian Jun, et al., "1.25 Gb/s Low Power CMOS Limiting Amplifier for Optical Receiver," 2004 Intl. Conf. on Solid-State and Integrated Circuits Tech., vol. 2, pp. 1469-1471, Oct. 18-21, 2004. 
14. Jong Hwa Lee, et al., "Design of a 2.5 GBPS CMOS Trans-Impedance Amplifier (TIA) for Optical Communication," the $8^{\text {th }}$ Russian-Korean Intl. Symp. on Science and Technology (KORUS 2004), vol. 1, pp. 229-233, June 26 - July 3, 2004.

15. Eduard Sachinger and Wilhelm C. Fischer, "A 3-GHz 32-dB CMOS Limiting Amplifier for SONET OC-48 Receivers," IEEE Jour. Of Solid-State Circuits, vol. 35, No. 12, Dec. 2000.

16. George E. Valley and Henry Wallman, Vacuum Tube Amplifiers (Radiation Lab Series, 18), McGraw Hill, 1948.

17. Hung Tien Bui, Yvon Savaria, "Shunt-Peaking of MCML Gates Using Active Inductors," the $2^{\text {nd }}$ Annual IEEE Northeast Workshop on Circuits and Systems (NEWCAS 2004), pp. 361 - 364, June 20-23, 2004.

18. J. H. C. Zhan, J. S. Duster, K. T. Kornegay, "A 7.3-GHz, 55\% Tuning Range Emitter Degenerated Active Inductor VCO," 2004 Bipolar/BiCMOS Circuits and Technology Meeting (BCTM), pp. 60 - 63, Sept. 13-14, 2004.

19. Jo-Yi Foo, Robert J. Weber, "Low Power 5 GHz Quadrature Phase CMOS LC Oscillator with Active Inductor," 2004 IEEE Intl. Symp. on Comm. and Info. Tech. Vol. 2, pp. 1084-1089, Oct. 26-29, 2004.

20. Johan van der Tang, et al., "A 9.8-11.5 GHz Quadrature Ring Oscillator for Optical Receivers," 2001 IEEE Conf. on Custom Integrated Circuits, pp. 323326, May 6-9, 2001.

21. Yanjie Wang, M. Zamin Khan, S. Masood Ali, et al., "A Fully Differential CMOS Limiting Amplifier with Active Inductor for Optical Receiver," 2005 Canadian Conf. on Electrical and Computer Engr., pp. 1751-1754, May 1-4, 2005.

22. Chia-Hsin Wu, Jieh-Wei Liao, Shen-Iuan Liu, "A 1 V $4.2 \mathrm{~mW}$ Fully Integrated $2.5 \mathrm{~Gb} / \mathrm{s}$ CMOS Limiting Amplifier using Folded Active Inductors," 2004 Intl. Symp. on Circuits and Systems (ISCAS 2004), vol. 1, pp. 1044-1047, May 2326, 2004.

23. Flora Carreto-Castro, Jose Silva-Martinez, Roberto Murphy-Arteaga, "RF LowNoise Amplifiers in BiCMOS Technologies," IEEE Trans. On Circuits and Systems - II, vol. 46, pp. 974-977, July 1999.

24. Shinji Hara, Tsuneo Tokumitsu, Toshiaki Tanaka, Masayoshi Aikawa, "Broadband Monolithic Microwave Active Inductor and Application to a Miniaturized Wide Band Amplifier," 1988 IEEE Microwave and MillimeterWave Monolithic circuits Symp., pp. 117-120, May 24-25, 1988. 
25. Shinji Hara, Tsuneo Tokumitsu, Toshiaki Tanaka, Masayoshi Aikawa, "Broadband Monolithic Microwave Active Inductor and Its Application to Miniaturized Wide-Band Amplifiers," IEEE Trans. On Microwave Theory and Techniques, vol. 36, No. 12, pp. 1920-1924, Dec. 1988.

26. Hitoshi Hayashi, et al., "A Novel Loss Compensation Technique for High-Q Broad-band Active Inductors," IEEE 1996 Microwave and Millimeter-Wave Monolithic Circuits Symp., pp. 103-106, June 16-18, 1996.

27. Hitoshi Hayashi, et al., "A High- $Q$ Broad-band Active Inductor and Its Application to a Low-Loss Analog Phase Shifter," IEEE Trans. On Microwave Theory and Techniques, vol. 44, No. 12, pp. 2369-2374, Dec. 1996.

28. Yong-Ho Cho, et al, "A Novel Active Inductor and Its Application to Inductance-Controlled Oscillator," IEEE Trans. On Microwave Theory and Techniques, vol. 45, No. 8, pp. 1208-1213, Aug. 1997.

29. Yongho Cho, et al., "Monolithic VCO using a Novel Active Inductor," IEEE 1996 Microwave and Millimeter-Wave Monolithic Circuits Symp., pp. 155-158, June 16-18, 1996.

30. Yongho Cho, et al., "Monolithic VCO using a Novel Active Inductor," 1996 IEEE MTT-S Intl. Microwave Symp., vol. 1, pp. 137-140, June 17-21, 1996.

31. W. D. Jemison, P. R. Herczfeld, "A Pulsed Microwave Oscillator Using Optically Controlled Active Feedback," IEEE Microwave and Guided Wave Letters, vol. 2, No. 5, pp. 177-179, May 1992.

32. Stepan Lucyszyn, Ian D. Robertson, "High Performance MMIC Narrow Band Filter using Tunable Active Inductors," IEEE 1994 Microwave and MillimeterWave Monolithic Circuits Symp., pp. 91-93, May 22-25, 1994.

33. Stepan Lucyszyn, Ian D. Robertson, "Monolithic Narrow-Band Filter using Ultrahigh- $Q$ Tunable Active Inductors," IEEE Trans. On Microwave Theory and Techniques, vol. 42, No. 12, pp. 2617-2622, Dec. 1994.

34. E. C. Krantz and G. R. Branner, "Synthesis of Active Microwave Filters Incorporating Noise Effects," $36^{\text {th }}$ Midwest Symp. on Circuits and Systems, vol. 2, pp. 805-808, Aug. 16-18, 1993.

35. R. Kaunisto, et al., "A Low-Power HBT MMIC Filter Based on Tunable Active Inductors," IEEE Microwave and Guided Wave Letters, vol. 7, No. 8, pp. 209211, Aug. 1997. 
36. Shinji Hara, Tsuneo Tokumitsu, Masayoshi Aikawa, "Lossless, Broadband Monolithic Microwave Active Inductors," 1989 IEEE MTT-S Intl. Microwave Symp. Digest, vol. 3, pp. 955-958, June 13-15, 1989.

37. Shinji Hara, Tsuneo Tokumitsu, Masayoshi Aikawa, "Lossless Broad-band Monolithic Microwave Active Inductors," IEEE Trans. On Microwave Theory and Techniques, vol. 37, No. 12, pp. 1979-1984, Dec. 1989.

38. Shinji Hara, Tsuneo Tokunitsu, "Monolithic Microwave Active Inductors and Their Applications," 1991 IEEE Symp. on Circuits and Systems, vol. 3, pp. 1857-1860, June 11-14, 1991.

39. Wei Li, et al., "High Q Active Inductor with Loss Compensation by Feedback Network," Electronic Letters, vol. 35, pp. 1328-1329, Aug. 1999.

40. $\quad$ Sung-Gi Yang, et al., "Fully Symmetrical, Differential-Pair Type Floating Active Inductors," 1997 IEEE Intl. Symp. on Circuits and Systems (ISCAS 1997), vol. 1, pp. 93-96, June 9-12, 1997.

41. Yue $\mathrm{Wu}$, Mohammed Ismail, and Hakan Olsson, "A Novel CMOS Fully Differential Inductorless RF Bandpass Filter," 2000 IEEE Intl. Symp. on Circuits and Systems, vol. 4, pp. 149-152, May 28-31, 2000.

42. Yue $\mathrm{Wu}$, Mohammed Ismail, and Hakan Olsson, "Inductorless CMOS RF Bandpass Filter," Electronic Letters, vol. 37, No. 16, pp. 1027-1029, August 2001.

43. Yue $\mathrm{Wu}$, Mohammed Ismail, and Hakan Olsson, "CMOS VHF/RF CCO Based on Active Inductors," Electronic Letters, vol. 37, No. 8, pp. 472-473, Apr. 2001.

44. Yue $\mathrm{Wu}$, Mohammed Ismail, and Hakan Olsson, "CMOS Active Inductor and Its Application in RF Bandpass Filter," 2004 IEEE Radio Frequency Integrated Circuits (RFIC) Symposium, pp. 655-658, June 6-8, 2004.

45. Yue Wu, Mohammed Ismail, and Hakan Olsson, "RF Bandpass Filter Design Based on CMOS Active Inductors," IEEE Trans. On Circuits and Systems-II, vol. 50, No. 12, pp. 942-949, Dec. 2003.

46. David Johns, Ken Martin, Analog Integrated Circuit Design, John Wiley and Sons, Inc., New York 1997.

47. Bosco H. Leung, VLSI for Wireless Communication, Pearson Education, 2002.

48. Jhy-Neng Yang, et al., "A Design of CMOS Broadband Amplifier with High-Q Active Inductor," $3^{\text {rd }}$ IEEE Intl. Workshop on System on Chip for Real Time Appl., pp. 86-89, June 30-July 2, 2003. 
49. Jhy-Neng Yang, et al., "A 1.75 GHz Inductorless CMOS Low Noise Amplifier with High-Q Active Inductor Load," 44 ${ }^{\text {th }}$ IEEE Midwest Symp. on Circuits and Systems (MWSCAS 2001), vol. 2, pp. 816-819, Aug. 14-17, 2001.

50. W. Zhuo, et al., "Programmable Low Noise Amplifier with Active-Inductor Load," 1998 IEEE Intl. Symp. on Circuits and Systems (ISCAS 1998), vol. 4, pp. 365-368, May 31-June 3, 1998.

51. Ming-Juei Wu, Jyh-Neng Yang, and Chen-Yi Lee, "A Constant Power Consumption CMOS LC Oscillator Using Improved High-Q Active Inductor with Wide Tuning Range," $47^{\text {th }}$ IEEE International Midwest Symp. on Circuits and Systems (MWSCAS 2004), vol. 3, pp. 347-500, July 25-28, 2004.

52. Chao-Chih Hsiao, et al., "Improved Quality-Factor of 0.18-um CMOS Active Inductor by a Feedback Resistance Design," IEEE Microwave and Wireless Components Letters, vol. 12, No. 12, pp. 467-469, Dec. 2002.

53. K. Manetakis, et al., "Wideband CMOS Analog Cells for Video and Wireless Communications," $3{ }^{\text {rd }}$ IEEE Intl. Conf. on Electronics, Circuits and Systems (ICECS 1996), vol. 1, pp. 227-230, Oct. 13-16, 1996.

54. U. Yodprasit and J. Ngarmnil, "Q-Enhancing Technique for RF CMOS Active Inductor," 2000 IEEE Intl. Symp. on Circuits and Systems (ISCAS 2000), vol. 5, pp. 589-592, May 28-31, 2000.

55. W. Zhuo, J. Pineda de Gyvez, E. Sanchez-Sinencio, "Programmable Low Noise Amplifier with Active-Inductor Load," 1998 IEEE Intl. Symp. on Circuits and Systems, vol. 4, pp. 365-368, May 31-June 3, 1998.

56. Chao-Chih Hsiao, et al., "Improved Quality-Factor of 0.18-um CMOS Active Inductor by a Feedback Resistance Design," IEEE Microwave and Wireless Components Letters, vol. 12, No. 12, pp. 467-469, Dec. 2002.

57. Jyh-Neng Yang, et al., “A 1.75 GHz Inductor-less CMOS Low Noise Amplifier with High-Q Active Inductor Load," $44^{\text {th }}$ IEEE Midwest Symp. on Circuits and Systems (MWSCAS 2001), vol. 2, pp. 816-819, Aug. 14-17, 2001.

58. Jyh-Neng Yang, et al., "A Design of CMOS Broadband Amplifier with High-Q Active Inductor," $3{ }^{\text {rd }}$ IEEE Intl. Workshop on System-on-Chip for Real-Time Applications, pp. 86-89, June 30-July 2, 2003.

59. Risto Kaunisto, Kari Stadius and Veikko Porra, “A 3 GHz Silicon-BJT Active Resonator and Filter," 1998 IEEE Intl. Conf. on Electronics, Circuits and Systems (ICECS 1998), vol. 3, pp. 197-200, Sept. 7-10, 1998. 
60. Apinunt Thanachayanont and Alison Payne, "VHF CMOS Integrated Active Inductor,” Electronic Letters, vol. 32, No. 11, pp. 999-1000, May 1996.

61. Rahim Akbari-Dilmaghani and Alilson Payne, "An RF CMOS Differential Bandpass Amplifier using an Active Inductor," IEE Colloquium on Systems on a Chip, pp. 16/1-16/6, Sept. 5, 1998.

62. R. Akbari-Dilmaghani, A. Payne and C. Toumazou, "A High Q RF CMOS Differential Active Inductor," 1998 IEEE Intl. Conf. on Electronics, Circuits and Systems (ICECS 1998), vol. 3, pp. 157-160, Sept. 7-10, 1998.

63. Rahim Akbari-Dilmaghani and Alison Payne, "An RF BiCMOS Bandpass Amplifier using an Active Inductor," $6{ }^{\text {th }}$ IEEE Intl. Conf. on Electronics, Circuits and Systems (ICECS 1999), vol. 2, pp. 611-614, Sept. 5-8, 1999.

64. Markus Grözing, et al., "A 2.5V CMOS Differential Active Inductor with Tunable L and Q for Frequencies up to $5 \mathrm{GHz}$," 2001 IEEE Radio Frequency Integrated Circuits (RFIC) Symposium, pp. 271-274, May 20-22, 2001.

65. Aydin Ilker Karsilayan and Rolf Schaumann, "A High-Frequency High-Q CMOS Active Inductor with dc Bias Control," 43 ${ }^{\text {rd }}$ IEEE Midwest Symp. on Circuits and Systems (MWSCAS 2000), vol. 1, pp.486-489, Aug. 8-11, 2000.

66. Haiqiao Xiao and Rolf Schaumann, "Very-High-Frequency Lowpass Filter Based on a CMOS Active Inductor," 2002 IEEE Intl. Symp. on Circuits and Systems (ISCAS 2002), Vol. 2, pp. 1-4, May 26-29, 2002.

67. H. Xiao and R. Schaumann, "A Low-Voltage Low-Power CMOS 5-GHz Oscillator Based on Active Inductors," 9th IEEE Intl. Conf. on Electronics, Circuits and Systems (ICECS 2002), Vol. 1, pp. 231-234, Sept. 15-18, 2002.

68. S. Sae-Ngow and A. Thanachayanont, "A \pm 1 Volt Very High Frequency Class AB CMOS Active Inductor," The $46^{\text {th }}$ IEEE Intl. Midwest Symp. on Circuits and Systems (MWSCAS'03), Vol. 1, pp. 157-160, Dec. 27-30, 2003.

69. S. Sae-Ngow and A. Thanachayanont, "A Low-Voltage, Wide Dynamic Range CMOS Floating Active Inductor," Conf. on Convergent Technologies for AsiaPacific Region (TENCON'03), Vol. 4, pp. 1460-1463, Oct. 15-17, 2003.

70. Apinunt Thanachayanont, "Low Voltage Low Power CMOS Inductorless RF Bandpass Filter with High Image Rejection Capability," The $45^{\text {th }}$ Midwest Symp. on Circuits and Systems (MWSCAS'02), Vol. 3, pp. 548-551, Aug. 4-7, 2002. 
71. Apinunt Thanachayanont, "2-V 3.36-mW 2.5-GHz Fourth-Order Inductorless CMOS RF Bandpass Filter," 2002 Asia-Pacific Conf. on Circuits and Systems (APCCAS'02), Vol. 1, pp. 127-130, Oct. 28-31, 2002.

72. Apinunt Thanachayanont and S. Sae-Ngow, "Low Voltage High-Q VHF CMOS Transistor-Only Active Inductor," The $45^{\text {th }}$ Midwest Symp. on Circuits and Systems (MWSCAS'02), Vol. 3, pp. 552-555, Aug. 4-7, 2002.

73. Apinunt Thanachayanont, "CMOS Transistor-Only Active Inductor for IF/RF Applications,” 2002 IEEE Conf. on Industrial Technology (ICIT'02), Vol. 2, pp. 1209-1212, Dec. 11-14, 2002.

74. Apinunt Thanachayanont and S. Sae-Ngow, "Class AB VHF CMOS Active Inductor," The $45^{\text {th }}$ Midwest Symp. on Circuits and Systems (MWSCAS'02), Vol. 1, pp. 64-7, Aug. 4-7, 2002.

75. Apinunt Thanachayanont, “A 1.5-V CMOS Fully Differential Inductorless RF Bandpass Amplifier,” 2001 IEEE Intl. Symp. on Circuits and Systems (ISCAS'01), Vol. 1, pp. 49-52, May 6-9, 2001.

76. Apinunt Thanachayanont, "Low Voltage CMOS Fully Differential Active Inductor and Its Application to RF Bandpass Amplifier Design," 2001 Intl. Symp. on VLSI Technology, Systems and Applications, pp. 125-28, Apr. 18-20, 2001.

77. Apinunt Thanachayanont and A. Payne, "CMOS Floating Active Inductor and Its Applications to Bandpass Filter and Oscillator Designs," IEE Proc. On Circuits, Devices and Systems, Vol. 147, pp. 42-48, Feb. 2000.

78. Apinunt Thanachayanont, "A 1.5-V High-Q CMOS Active Inductor for IF/RF Wireless Applications," 2000 Asia-Pacific Conf. on Circuits and Systems (APCCAS’00), pp. 654-57, Dec. 4-6, 2000.

79. Apinunt Thanachayanont and Alison Payne, "A 3-V RF CMOS Bandpass Amplifier Using an Active Inductor," 1998 IEEE Intl. Symp. on Circuits and Systems (ISCAS'98), Vol. 1, pp. 440-443, May 31 - June 3, 1998.

80. Haiqiao Xiao, Rolf Schaumann and W. Robert Daasch, High-Frequency Active Inductor, US Patent 7,042,317, Sept. $8^{\text {th }}, 2004$.

81. H. Xiao, Schaumann, et al., "A Radio-Frequency CMOS Active Inductor and Its Application in Designing High-Q Filters," IEEE Intl. Symp. on Circuits and Systems (ISCAS2004), Vol. 4, pp. 197-200, May 23-26, 2004, Vancouver, Canada. 
82. Haiqiao Xiao and Rolf Schaumann, "A 5.4-GHz High-Q Tunable ActiveInductor Bandpass Filter in Standard Digital CMOS Technology," Analog Integrated Circuits and Signal Processing, Vol. 51, pp. 1-9, April 2007.

83. Thomas H. Lee, The Design of CMOS Radio-Frequency Integrated Circuits, $2^{\text {nd }}$ Ed., Cambridge Univ. Press., 2003.

84. B. Razavi, "Design of Analog CMOS Integrated Circuits," McGraw-Hill, 2000.

85. D. M. Pozar, Microwave Engineering, Wiley, John and Sons, Inc., 2004.

86. Guillermo Gonzalez, Microwave Transistor Amplifiers, $2^{\text {nd }}$ edition, Prentice Hall, Upper Saddle River, New Jersey, 1997.

87. Behzad Razavi, "A Study of Phase Noise in CMOS Oscillators," IEEE J. of Solid-State Circuits, Vol. 31, No. 3, pp. 331-343, March 1996.

88. D. B. Leeson, "A Simple Model of Feedback Oscillator Noise Spectrum," Proc. Of IEEE, Vol. 54, No. 2, pp. 329-330, 1966.

89. Asad A. Abidi, Analog Circuit Design - RF Analog-to-Digital Converters; Sensor and Actuator Interfaces; Low-Noise Oscillators, PLLs and Synthesizers, Kluwer Academic Publishers, Boston, Nov. 1997.

90. Asad A. Abidi, "Phase Noise and Jitter in CMOS Ring Oscillators," IEEE J. of Solid-State Circuits, Vol. 41, No. 8, pp. 1803-1815, Aug. 2006.

91. Cadence SpectreRF® User’s Manual, Cadence Design Systems, San Jose, CA.

92. P. R. Gray, P. J. Hurst, S. H. Lewis, and R. G. Meyer, Analysis and Design of Analog Integrated Circuits, $4^{\text {th }}$ Ed., John Wiley and Sons, Inc., New York, 2001.

93. Alan Hastings, Art of Analog Layout, $2^{\text {nd }}$ Ed., Prentice Hall, 2005.

94. Slawomir Koziel, Rolf Schaumann and Haiqiao Xiao, "Analysis and Optimization of Noise in Continuous-Time OTA-C Filters," IEEE Transactions on Circuits and Systems, Vol. 52, pp. 1086-94, June 2005. 